\title{
Theory of Neutrinos: A White Paper
}

\author{
R.N. Mohapatra* (Group Leader)
}

S. Antusch ${ }^{1}$, K.S. Babu ${ }^{2}$, G. Barenboim ${ }^{3}$, M.-C. Chen ${ }^{4}$, S. Davidson ${ }^{5}$, A. de Gouvêa ${ }^{6}$, P. de Holanda ${ }^{7}$, B. Dutta ${ }^{8}$, Y. Grossman ${ }^{9}$, A. Joshipura ${ }^{10}$, B. Kayser ${ }^{11}$, J. Kersten ${ }^{12}$, Y.Y. Keum ${ }^{13}$, S.F. King ${ }^{14}$ P. Langacker ${ }^{15}$, M. Lindner ${ }^{16}$, W. Loinaz ${ }^{17}$, I. Masina ${ }^{18}$, I. Mocioiu ${ }^{19}$, S. Mohanty ${ }^{10}$, H. Murayama ${ }^{20}$, S. Pascoli ${ }^{21}$, S.T. Petcov ${ }^{22,23}$, A. Pilaftsis ${ }^{24}$, P. Ramond ${ }^{25}$, M. Ratz ${ }^{26}$, W. Rodejohann ${ }^{16}$, R. Shrock ${ }^{27}$, T. Takeuchi ${ }^{28}$, T. Underwood ${ }^{5}$, L. Wolfenstein ${ }^{29}$

* University of Maryland, College Park, MD 20742, USA

${ }^{1}$ Universidad Autónoma de Madrid, 28049 Madrid, Spain and University of Southampton, Southampton SO17 1BJ, United Kingdom

${ }^{2}$ Oklahoma State University, Stillwater, OK-74078, USA

${ }^{3}$ University of Valencia, Valencia, Spain

${ }^{4}$ Fermilab, Batavia, IL 60540

${ }^{5}$ IPPP, University of Durham, Durham, DH1 3LE, Great Britain

${ }^{6}$ Northwestern University, Evanston, Illinois 60208-3112, USA

${ }^{7}$ Instituto de Física Gleb Wataghin, UNICAMP PO BOX 6165, CEP 13083-970, Campinas - SP, Brazil

${ }^{8}$ University of Regina, Regina, Saskatchewan, Canada

${ }^{9}$ SLAC, Stanford, CA-94305, USA

${ }^{10}$ Physical Research Laboratory, Ahmedabad 380009, India

${ }^{11}$ Fermilab, Batavia, Il-60617, USA

12 Deutsches Elektronen-Synchrotron DESY, 22603 Hamburg, Germany

${ }^{13}$ Institute of Physics, Academia Sinica, Taipei, Taiwan 115, Republic of China

${ }^{14}$ University of Southampton, Southampton SO17 1BJ, United Kingdom

${ }^{15}$ University of Pennsylvania, Philadelphia, PA 19104-6396, USA

16 Technische Universität München, James-Franck-Straße, 85748 Garching, Germany

17 Amherst College, Amherst, MA 01002-5000, USA

${ }^{18}$ Fermi Center, Via Panisperna 89/A, I-00184 Roma, Italy

and INFN, Sezione di Roma, "La Sapienza" Univ., P.le A. Moro 2, I-00185 Roma, Italy

${ }^{19}$ Pennsylvania State University, University Park, PA 16802, USA

${ }^{20}$ School of Natural Sciences, Institute for Advanced Study, Princeton, NJ 08, USA ${ }^{1}$

${ }^{21}$ UCLA, Los Angeles, CA 90095-1547, USA and Department of Physics, Theory

Division, CERN, CH-1211 Geneva 23, Switzerland

\footnotetext{
${ }^{1}$ On leave of absence from Department of Physics, University of California, Berkeley, CA 94720.
} 
${ }^{22}$ SISSA/INFN-sezione di Trieste, Trieste, Italy

23 INRNE, Bulgarian Academy of Sciences, Sofia, Bulgaria

24 School of Physics and Astronomy, University of Manchester, Manchester M13 9PL, United Kingdom

25 University of Florida, Gainesville, FL 32611, USA

26 Physikalisches Institut der Universität Bonn, Nussallee 12, 53115 Bonn, Germany

27 Department of Physics, Sloan Laboratory, Yale University, New Haven, CT 06250, USA

28 Virginia Tech, Blacksburg, VA 24061, USA

${ }^{29}$ Carnegie-Mellon University, Pittsburgh, PA 15213, USA

\begin{abstract}
During 2004, four divisions of the American Physical Society commissioned a study of neutrino physics to take stock of where the field is at the moment and where it is going in the near and far future. Several working groups looked at various aspects of this vast field. The summary was published as a main report entitled "The Neutrino Matrix" accompanied by short 50 page versions of the report of each working group. Theoretical research in this field has been quite extensive and touches many areas and the short 50 page report [1] provided only a brief summary and overview of few of the important points. The theory discussion group felt that it may be of value to the community to publish the entire study as a white paper and the result is the current article. After a brief overview of the present knowledge of neutrino masses and mixing and some popular ways to probe the new physics implied by recent data, the white paper summarizes what can be learned about physics beyond the Standard Model from the various proposed neutrino experiments. It also comments on the impact of the experiments on our understanding of the origin of the matter-antimatter asymmetry of the Universe and the basic nature of neutrino interactions as well as the existence of possible additional neutrinos. Extensive references to original literature are provided.
\end{abstract}




\section{Contents}

$\mathbf{1}$ Introduction $\quad \mathbf{6}$

1.1 Our present knowledge about masses and mixings . . . . . . . . . . . . . . 7

1.1.1 Dirac versus Maiorana Neutrinos . . . . . . . . . . . . . . 7

1.1 .2 Neutrino mixings . . . . . . . . . . . . . . . . . . . . 8

1.1.3 Matter effect on neutrino propagation . . . . . . . . . . . . . . . . 9

1.1 .4 Neutrino masses . . . . . . . . . . . . . . . . . . . . . . . . 9 9

1.1 .5 Overall scale for masses . . . . . . . . . . . . . . . . 10

1.1.6 Neutrino masses and neutrinoless double beta decav . . . . . . . . . 10

1.1.7 Cosmology and neutrino masses . . . . . . . . . . . . . . . . 11

1.1 .8 CP violation . . . . . . . . . . . . . . . . . . 12

1.1.9 Prospects for determining whether neutrinos are Majorana or Dirad 12

1.1 .10 Sterile neutrinos . . . . . . . . . . . . . . . 12

1.1.11 Neutrino electromagnetic dipole moments and neutrino decav . . . 14

1.2 Neutrino probes of other fundamental svmmetries . . . . . . . . . . 15

1.3 Why neutrino mass necessarily means phvsics bevond the Standard Model? 15

1.3.1 Seesaw mechanism for small neutrino masses . . . . . . . . . . . 16

1.3.2 Tvpe I vs tvpe II seesaw mechanism . . . . . . . . . . . . . . . . 17

1.3 .3 Triplet seesaw . . . . . . . . . . . . . . . . . . 17

1.3.4 Seesaw with triplet fermions . . . . . . . . . . . . . . . . 17

1.3.5 Understanding large mixings within the seesaw mechanism . . . . . 18

1.3.6 Alternatives to high-scale seesaw . . . . . . . . . . . . . . 19

1.4 Summary of the Introduction . . . . . . . . . . . . . . . . . . . . . 19

2 What can we learn about neutrino mass matrices from experiments? 21

$\begin{array}{lll}3 & \boldsymbol{\beta} \beta_{0 \nu} \text {-Decay and CP Violation } & 28\end{array}$

$3.1 \beta \beta_{0,}$ decav . . . . . . . . . . . . . . . . . . . 28

3.2 The MNSP Lepton Mixing Matrix and CP Violation in the Lepton Secton 32

4 Testing Seesaw Models 35

4.1 Lepton Flavor Violation and Lepton Electric Dipole Moments . . . . . . . 35

4.1 .1 Tvpe I Seesaw: LFV . . . . . . . . . . . . . . . . . . . . . 36

4.1 .2 Tvpe I Seesaw: EDM . . . . . . . . . . . . . . . . . . . . . . . . . 38

4.1 .3 Tvpe II Seesaw . . . . . . . . . . . . . . . . . . . . . 38

4.2 Leptogenesis in the Tvpe I Seesaw . . . . . . . . . . . . . . . . . . . 42

4.2 .1 Thermal Leptogenesis . . . . . . . . . . . . . . . . . . 42

4.2 .2 Parametrizing the tvpe I seesaw . . . . . . . . . . . . . . . 44

4.2 .3 Implications for $\mathrm{CP}$ conserving observables . . . . . . . . . . . . 44

4.2.4 Relations between Leptogenesis and leptonic CP violation . . . . . 45

4.2 .5 Model dependent approaches. . . . . . . . . . . . . . . . . . . 46

4.2 .6 Leptogenesis in supersvmmetric scenarios . . . . . . . . . . . . 46

4.3 Leptogenesis and tvpe II seesaw mechanism . . . . . . . . . . . . . 47

4.4 Dirac Leptogenesis . . . . . . . . . . . . . . . . . . . . . . . . 50

4.5 Resonant Leptogenesis . . . . . . . . . . . . . . . . . . . . . . . . . 52 
5 The Heavy Majorana Mass Matrix $\quad 56$

5.1 General Considerations . . . . . . . . . . . . . . . . . . 56

5.1.1 The Three Right-Handed Neutrino Paradiom . . . . . . . . . . . 56

5.1 .2 Grand Unification . . . . . . . . . . . . . . . . . . . . . . 58

5.1 .3 Leptogenesis . . . . . . . . . . . . . . . . . . . . . . . . 59

5.1 .4 Sneutrino Inflation . . . . . . . . . . . . . . . . . . 59

5.1 .5 Tvpe II Seesaw Models . . . . . . . . . . . . . . . . . . . . 59

5.1 .6 Right-Handed Neutrinos in Extended Technicolor . . . . . . . . . . 60

5.2 Seesaw Neutrino mass and Grand unification . . . . . . . . . . . . 60

5.2 .1 A minimal 126-based $S O(10)$ model . . . . . . . . . . . . . 61

5.2 .2 16-based models . . . . . . . . . . . . . . . . . . . 62

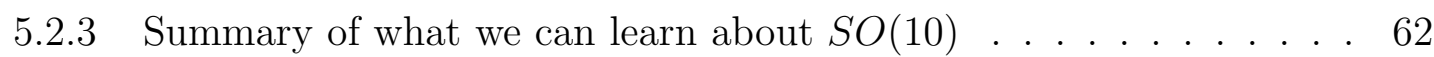

5.2.4 Implications of Models with Spontaneous CP Violation . . . . . . . 63

5.3 Renormalization group evolution of neutrino parameters . . . . . . . . . 64

5.3.1 Running masses. mixings and CP phases below the seesaw scale . . 64

5.3.2 Details of the running in seesaw models . . . . . . . . . . . . 65

5.3 .3 Implications for model building . . . . . . . . . . . . . . . 65

5.3.4 High-scale mixing unification and large mixings . . . . . . . . . 66

5.3.5 Deviations of $\theta_{13}$ from 0 and of $\theta_{23}$ from $\pi / 4$ due to RG effects . . . 67

5.3 .6 Implications for leptogenesis . . . . . . . . . . . . . . . . 67

6 Non-Standard Neutrino Interactions $\quad 68$

6.1 Neutrino magnetic moments . . . . . . . . . . . . . . . . . . . 68

6.2 Flavor changing and conserving nonstandard neutral current interactions . 70

6.2 .1 Atmospheric neutrinos . . . . . . . . . . . . . . 70

6.2 .2 KamLAND and solar neutrinos . . . . . . . . . . . . . . 71

6.2 .3 Bounds from non-oscillating phenomena $\ldots \ldots \ldots$. . . . . 72

$\begin{array}{lll}7 & \text { Bevond the three neutrino picture } & 74\end{array}$

7.1 The search for other light neutrinos . . . . . . . . . . . . . . . . 74

7.1 .1 Issues of theoretical iustification . . . . . . . . . . . . 74

7.1.2 Phenomenological manifestations . . . . . . . . . . . 75

7.1.3 Summarv of what we can learn on light sterile neutrinos . . . . . 78

7.2 What can we learn about four-neutrino mass matrices . . . . . . . . . 78

$7.3 \quad$ Heavv sterile neutrinos . . . . . . . . . . . . . . . . . . . . . . 80

7.3 .1 Laboratorv Experiments . . . . . . . . . . . . . . . . . 81

7.3 .2 Astrophvsics and cosmology . . . . . . . . . . . . . . 82

8 Supersvmmetrv and neutrinos $\quad 85$

8.1 Neutrino masses from R-paritv violation . . . . . . . . . . . . . . . 85

8.2 Neutrino masses from supersvmmetrv breaking . . . . . . . . . . . . . . . 86

8.3 Sneutrino oscillation $\ldots \ldots \ldots \ldots \ldots$

8.4 Sneutrino flavor oscillation . . . . . . . . . . . . . . . . . . 87

$\begin{array}{lll}9 & \text { Expectations in Superstring Constructions } & 89\end{array}$ 
10 Theories with a TeV-scale $U(1)$

11 Neutrino Masses in Theories with Dvnamical Svmmetrv Breaking 93

$\begin{array}{lr}12 \text { Neutrinos in extra dimensions } & 95\end{array}$

12.1 Three bulk neutrinos . . . . . . . . . . . . . . . . . . . . . . 96

12.2 Lepton number breaking in the bulk . . . . . . . . . . . . . . . . 97

$\begin{array}{ll}13 \text { Other new physics and neutrinos } & 99\end{array}$

13.1 New long range forces . . . . . . . . . . . . . . . . . . . . . . . . . . . . 99

13.2 Lorentz noninvariance. CPT violation and decoherence . . . . . . . . . . . 101

13.2 .1 CPT Violation . . . . . . . . . . . . . . . . . . . . . 101

13.2 .2 Decoherence . . . . . . . . . . . . . . . . . . . . . 103

$\begin{array}{ll}14 \text { NuTeV Physics } & 107\end{array}$

$\begin{array}{ll}15 \text { Conclusions } & 111\end{array}$ 


\section{Introduction}

Our understanding of neutrinos has changed dramatically in the past six years. Thanks to many neutrino oscillation experiments involving solar, atmospheric, accelerator and reactor (anti)-neutrinos [2,3], we have learned that neutrinos produced in a well defined flavor eigenstate can be detected, after propagating a macroscopic distance, as a different flavor eigenstate. The simplest interpretation of this phenomenon is that, like all charged fermions, the neutrinos have mass and that, similar to quarks, the neutrino weak, or flavor, eigenstates are different from neutrino mass eigenstates i.e., neutrinos mix. This new state of affairs has also raised many other issues [4] which did not exist for massless neutrinos: For example, (i) massive Dirac neutrinos, like charged leptons and quarks, can have nonzero magnetic dipole moments and massive Dirac and Majorana neutrinos can have nonzero transition dipole moments; (ii) the heavier neutrinos decay into lighter ones, like charged leptons and quarks, and (iii) (most importantly) the neutrinos can be either Majorana or Dirac fermions (see later for details).

Learning about all these possibilities can not only bring our knowledge of neutrinos to the same level as that of charged leptons and quarks, but may also lead to a plethora of laboratory as well as astrophysical and cosmological consequences with far-reaching implications. Most importantly, knowing neutrino properties in detail may also play a crucial role in clarifying the blueprint of new physical laws beyond those embodied in the Standard Model.

One may also consider the possibility that there could be new neutrino species beyond the three known ones $\left(\nu_{e}, \nu_{\mu}, \nu_{\tau}\right)$. In addition to being a question whose answer would be a revolutionary milestone pointing to unexpected new physics, it may also become a necessity if the LSND results are confirmed by the MiniBooNE experiment, now in progress at Fermilab. This would, undoubtedly, be a second revolution in our thinking about neutrinos and the nature of unification.

The existence of neutrino masses qualifies as the first evidence of new physics beyond the Standard Model. The answers to the neutrino-questions mentioned above will add substantially to our knowledge about the precise nature of this new physics, and in turn about the nature of new forces beyond the Standard Model. They also have the potential to unravel some of the deepest and most long-standing mysteries of cosmology and astrophysics, such as the origin of matter, the origin of the heavy elements, and, perhaps, even the nature of dark energy.

Active endeavors are under way to launch the era of precision neutrino measurement science, that will surely broaden the horizon of our knowledge about neutrinos. We undertake this survey to pin down how different experimental results expected in the coming decades can elucidate the nature of neutrinos and our quest for new physics. In particular, we would like to know (i) the implications of neutrinos for such longstanding ideas as grand unification, supersymmetry, string theory, extra dimensions, etc; (ii) the implications of the possible existence of additional neutrino species for physics and cosmology, and (iii) whether neutrinos have anything to do with the origin of the observed matter-antimatter asymmetry in the universe and, if so, whether there is any way to determine this via low-energy experiments. Once the answers to these questions are at hand, we will have considerably narrowed the choices of new physics, providing a 
giant leap in our understanding of the physical Universe.

This review grew out of a year long study of the future of neutrino physics conducted by four divisions of the American Physical Society and is meant to be an overview of where we stand in neutrino physics today, ${ }^{2}$ where we are going in the next decades and the implications of this new knowledge for the nature of new physics and for the early universe. We apologize for surely missing vast parts of the neutrino literature in our references. We expect this overview to be supplemented by other excellent existing reviews of the subject in the literature. Regarding more references and the more experimental aspects of the topics under study, we refer to the other working group reports, the Solar and Atmospheric Experiments [5], the Reactor [6], the Neutrino Factory and Beta Beam Experiments and Development [7], the Neutrinoless Double Beta Decay and Direct Searches for Neutrino Mass [8] and the Neutrino Astrophysics and Cosmology [9] WGs. In particular, we have not discussed theoretical models for neutrino masses except giving a broad outline of ideas and getting beyond it only when there is a need to make some phenomenological point. Nonetheless, we hope to have captured in this study the essential issues in neutrino physics that will be relevant as we proceed to the next level in our exploration of this fascinating field.

\subsection{Our present knowledge about masses and mixings}

\subsubsection{Dirac versus Majorana Neutrinos}

The fact that the neutrino has no electric charge endows it with certain properties not shared by the charged fermions of the Standard Model. One can write two kinds of Lorentz invariant mass terms for the neutrino, Dirac and Majorana masses, whereas for the charged fermions, conservation of electric charge allows only Dirac-type mass terms. In the four component notation for describing fermions, commonly used for writing the Dirac equation for the electron, the Dirac mass has the form $\bar{\psi} \psi$, connecting fields of opposite chirality, whereas the Majorana mass is of the form $\psi^{T} C^{-1} \psi$ connecting fields of the same chirality, where $\psi$ is the four component spinor and $C$ is the charge conjugation matrix. In the first case, the fermion $\psi$ is different from its antiparticle, whereas in the latter case it is its own antiparticle. A Majorana neutrino implies a whole new class of experimental signatures, the most prominent among them being the process of neutrinoless double beta decay of heavy nuclei, $\left(\beta \beta_{0 \nu}\right)$. Since $\beta \beta_{0 \nu}$ arises due to the presence of neutrino Majorana masses, a measurement of its rate can provide very precise information about neutrino masses and mixing, provided (i) one can satisfactorily eliminate other contributions to this process that may arise from other interactions in a full beyond-the-standard-model theory, as we discuss below, (ii) one can precisely estimate the values of the nuclear matrix elements associated with the $\beta \beta_{0 \nu}$ in question.

The expressions for the Dirac and Majorana mass terms make it clear that a theory forbids Majorana masses for a fermion only if there is an additional global symmetry under which it has nonzero charge. As noted above, for charged fermions such as the electron and the muon, Majorana mass-terms are forbidden by the fact that they have nonzero

\footnotetext{
${ }^{2}$ The bulk of this report was finalized at the end of January 2005. We have not included the (sometimes substantial) progress that has been obtained in several areas of neutrino physics since then.
} 
electric charge and the theory has electromagnetic $U(1)$ invariance. Hence all charged fermions are Dirac fermions. On the other hand, a Lagrangian with both Majorana and Dirac masses describes, necessarily, a pair of Majorana fermions, irrespective of how small the Majorana mass term is (although it may prove very difficult to address whether the fermion is of the Dirac or the Majorana type when the Majorana mass-term is significantly smaller than the Dirac mass term). Hence, since the neutrino has no electric charge, the "simplest" theories predict that the neutrino is a Majorana fermion meaning that a Majorana neutrino is more natural (or at least requires fewer assumptions) than a Dirac neutrino. In most of the discussions below we assume that the neutrino is a Majorana fermion, unless otherwise noted.

\subsubsection{Neutrino mixings}

We will use a notation where the electroweak-doublet neutrino eigenstate (defined as the neutrino that is produced in a charged-current weak interaction process associated with a well-defined charged lepton) is denoted by $\nu_{\alpha}$, with $\alpha=e, \mu, \tau$. We will also consider $\nu_{\alpha}$ to include a set of $n_{s}$ possible electroweak-singlet ("sterile") neutrinos. Corresponding to these $3+n_{s}$ neutrino interaction eigenstates are $3+n_{s}$ mass eigenstates of neutrinos, $\nu_{i}$. We will order the basis of mass eigenstates so that $m_{1}^{2}<m_{2}^{2}$ and $\Delta m_{12}^{2}<\left|\Delta m_{13}^{2}\right|$, where $\Delta m_{i j}^{2} \equiv m_{j}^{2}-m_{i}^{2}$. The neutrino interaction eigenstates are expressed in terms of the mass eigenstates as follows: $\nu_{\alpha}=\sum_{i} U_{\alpha i} \nu_{i}$, where $U$ is a $\left(3+n_{s}\right) \times\left(3+n_{s}\right)$ dimensional unitary matrix. For the active neutrinos, with $\alpha=e, \mu, \tau$, the relevant submatrix is thus a rectangular matrix with three rows and $3+n_{s}$ columns. In seesaw models, the entries in the columns $4, \ldots 3+n_{s}$ are very small, of order $m_{D} / m_{R}$, where $m_{D}$ is a typical Dirac mass and $m_{R}$ is a large mass of a right-handed Majorana neutrino. Motivated by these models, one commonly assumes a decoupling, so that to good approximation the electroweakdoublet neutrinos can be expressed as linear combinations of just three mass eigenstates, and hence one deals with a $3 \times 3$ truncation of the full $\left(3+n_{s}\right) \times\left(3+n_{s}\right)$ neutrino mixing matrix. Since only the three electroweak-doublet neutrinos couple to the $W$, the actual observed lepton mixing matrix that appears in the charged weak current involves the product of the $3 \times\left(3+n_{s}\right)$ rectangular submatrix of the full lepton mixing matrix with the adjoint of the $3 \times 3$ unitary transformation mapping the mass to weak eigenstates of the charged leptons. Thus, the lepton mixing matrix occurring in the charge-lowering weak current has three rows and $3+n_{s}$ columns, corresponding to the fact that, in general, a charged lepton $\alpha$ couples to a $\nu_{\alpha}$ which is a linear combination of $3+n_{s}$ mass eigenstates. Henceforth, unless explicitly indicated, we shall assume the above-mentioned decoupling, so that the neutrino mixing matrix is $3 \times 3$, and will use $U$ to refer to the observed lepton mixing matrix, incorporating both the mixings in the neutrino and charged lepton sector. Neutrino oscillations and the mixing of two mass eigenstates of neutrinos, $\nu_{1}$ and $\nu_{2}$, to form the weak eigenstates $\nu_{e}$ and $\nu_{\mu}$ were first discussed by Pontecorvo and by Maki, Nakagawa, and Sakata [10]. The $3 \times 3$ truncation of the full neutrino mixing matrix is often called the MNS, MNSP, or PMNS matrix in honor of these pioneers.

For the case of three Majorana neutrinos, the lepton mixing matrix $U$ can be written 
as $V K$, where $V$ will be parametrized as

$$
V=\left(\begin{array}{ccc}
c_{12} c_{13} & s_{12} c_{13} & s_{13} e^{-i \delta} \\
-s_{12} c_{23}-c_{12} s_{23} s_{13} e^{i \delta} & c_{12} c_{23}-s_{12} s_{23} s_{13} e^{i \delta} & s_{23} c_{13} \\
s_{12} s_{23}-c_{12} c_{23} s_{13} e^{i \delta} & -c_{12} s_{23}-s_{12} c_{23} s_{13} e^{i \delta} & c_{23} c_{13}
\end{array}\right)
$$

while $K=\operatorname{diag}\left(1, e^{i \phi_{1}}, e^{i\left(\phi_{2}+\delta\right)}\right)[11,12]$.

Neutrino oscillation experiments have already provided measurements for the neutrino mass-squared differences, as well as the mixing angles. At the $3 \sigma$ level, the allowed ranges are [13] $\sin ^{2} 2 \theta_{23} \geq 0.87 ; 1.4 \times 10^{-3} \mathrm{eV}^{2} \leq\left|\Delta m_{13}^{2}\right| \leq 3.3 \times 10^{-3} \mathrm{eV}^{2} ; 0.70 \leq \sin ^{2} 2 \theta_{12} \leq 0.94$; $7.1 \times 10^{-5} \mathrm{eV}^{2} \leq \Delta m_{12}^{2} \leq 8.9 \times 10^{-5} \mathrm{eV}^{2} ; \sin ^{2} \theta_{13} \leq 0.051$ [14]. There is currently no constraint on any of the CP odd phases or on the sign of $\Delta m_{13}^{2}$. Note that in contrast to the quark sector we have two large angles (one possibly maximal) and one small (possibly zero) angle.

\subsubsection{Matter effect on neutrino propagation}

A very important fact about neutrinos that we seem to have learned from solar neutrino data is that neutrino propagation in matter is substantially different from that in vacuum. This effect is known as the MSW (Mikheev-Smirnov-Wolfenstein) effect [15] and has been widely discussed in the literature [16]. There is however an important aspect of the favored large mixing angle (LMA) MSW solution which needs to be tested in future experiments. The LMA solution predicts a rise in the survival probability in the energy region of a few $\mathrm{MeV}$ as we move down from higher to lower solar neutrino energies. Since the present data do not cover this energy region, new solar neutrino data is needed in order to conclusively establish the LMA solution [17].

\subsubsection{Neutrino masses}

Given the current precision of neutrino oscillation experiments and the fact that neutrino oscillations are only sensitive to mass-squared differences, three possible arrangements of the neutrino masses are allowed:

(i) Normal hierarchy, i.e. $m_{1}<m_{2} \ll m_{3}$. In this case $\Delta m_{23}^{2} \equiv m_{3}^{2}-m_{2}^{2}>0$, and $m_{3} \simeq \sqrt{\Delta m_{23}^{2}} \simeq 0.03-0.07 \mathrm{eV}$. The solar neutrino oscillation involves the two lighter levels. The mass of the lightest neutrino is unconstrained. If $m_{1} \ll m_{2}$, then we find the value of $m_{2} \simeq 0.008 \mathrm{eV}$.

(ii) Inverted hierarchy, i.e. $m_{1} \simeq m_{2} \gg m_{3}$ [18] with $m_{1,2} \simeq \sqrt{\Delta m_{23}^{2}} \simeq 0.03-0.07 \mathrm{eV}$. In this case, solar neutrino oscillation takes place between the heavier levels and we have $\Delta m_{23}^{2} \equiv m_{3}^{2}-m_{2}^{2}<0$. We have no information about $m_{3}$ except that its value is much less than the other two masses.

(iii) Degenerate neutrinos [19] i.e., $m_{1} \simeq m_{2} \simeq m_{3}$.

The behaviors of masses for different mass patterns are shown in Fig. 1. 

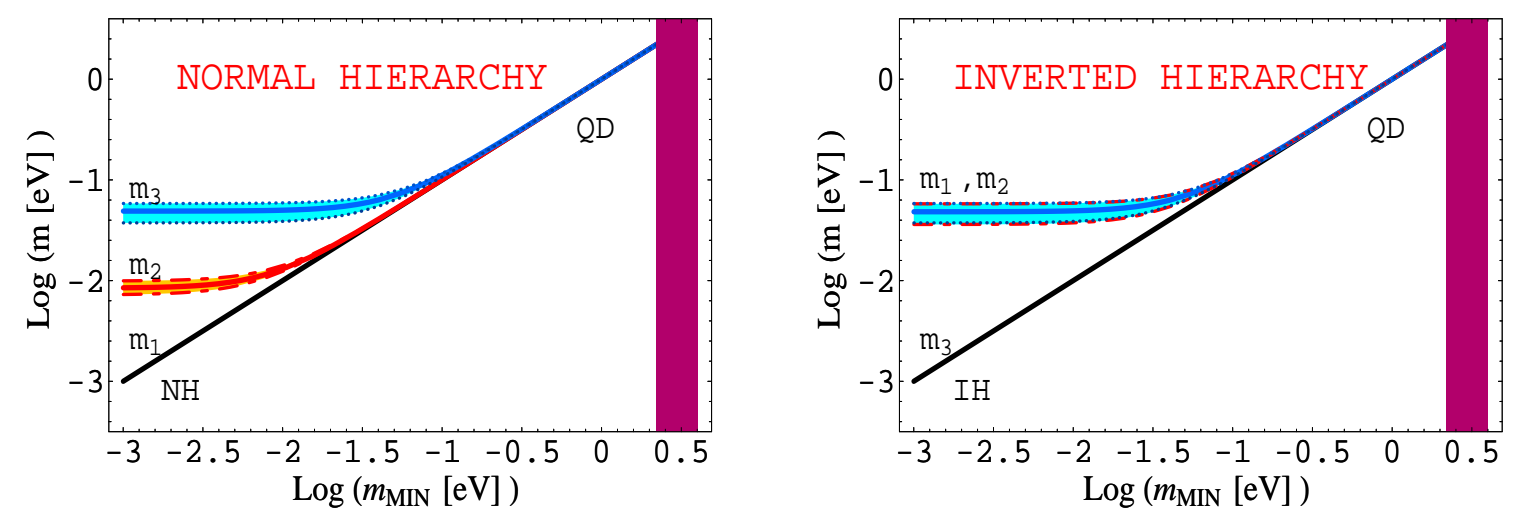

Figure 1: The three light neutrino masses as a function of the lightest mass for the normal (left plot) and inverted (right plot) hierarchy.

\subsubsection{Overall scale for masses}

Oscillation experiments cannot tell us about the overall scale of masses. It is therefore important to explore to what extent the absolute values of the masses can be determined. While discussing the question of absolute masses, it is good to keep in mind that none of the methods discussed below can provide any information about the lightest neutrino mass in the cases of a normal or inverted mass-hierarchy. They are most useful for determining absolute masses in the case of degenerate neutrinos i.e., when all $m_{i} \geq 0.1 \mathrm{eV}$.

Neutrino mass from beta decay

One can directly search for the kinematic effect of nonzero neutrino masses in betadecay by modifications of the Kurie plot. This search is sensitive to neutrino masses regardless of whether the neutrinos are Dirac or Majorana particles. These may be due to the emission, via mixing, of massive neutrinos that cause kinks in this plot. If the masses are small, then the effects will occur near to the end point of the electron energy spectrum and will be sensitive to the quantity $m_{\beta} \equiv \sqrt{\sum_{i}\left|U_{e i}\right|^{2} m_{i}^{2}}$. The Mainz [20] and Troitsk [21] experiments place the present upper limit on $m_{\beta} \leq 2.3 \mathrm{eV}$ and $2.2 \mathrm{eV}$, respectively. The proposed KATRIN [22] experiment is projected to be sensitive to $m_{\beta}>0.2 \mathrm{eV}$, which will have important implications for the theory of neutrino masses. For instance, if the result is positive, it will imply a degenerate spectrum; on the other hand a negative result will be a very useful constraint.

\subsubsection{Neutrino masses and neutrinoless double beta decay}

Another sensitive probe for the absolute scale of the neutrino masses is the search for neutrinoless double beta decay, $\beta \beta_{0 \nu}$, whose rate is potentially measurable if the neutrinos are Majorana fermions and $m_{e e}=\sum U_{e i}^{2} m_{i}$ is large enough [23,24], or if there are new lepton number violating interactions [25]. In the absence of new lepton number violating interactions, a positive sign of $\beta \beta_{0 \nu}$ would allow one to measure $m_{e e}$. Either way, we would learn that the neutrinos are Majorana fermions [26]. However, if $m_{e e}$ is very small, and there are new lepton number violating interactions, neutrinoless double beta decay will measure the strength of the new interactions (such as doubly charged Higgs fields 
or R-parity violating interactions) rather than neutrino mass. There are many examples of models where new interactions can lead to a $\beta \beta_{0 \nu}$ decay rate in the observable range without at the same time yielding a significant Majorana mass for the neutrinos. As a result, one must be careful in interpreting any nonzero signal in $\beta \beta_{0 \nu}$ experiments and not jump to the conclusion that a direct measurement of neutrino mass has been made. The way to tell whether such a nonzero signal is due to neutrino masses or is a reflection of new interactions is to supplement $\beta \beta_{0 \nu}$ decay results with collider searches for these new interactions. Thus collider experiments, such as those at LHC, and double beta experiments play complementary roles.

The present best upper bounds on $\beta \beta_{0 \nu}$ decay lifetimes come from the HeidelbergMoscow [27] and the IGEX [28] experiments and can be translated into an upper limit on $m_{e e} \lesssim 0.9 \mathrm{eV}$ [29]. There is a claim of discovery of neutrinoless double beta decay of enriched ${ }^{76} \mathrm{Ge}$ experiment by the Heidelberg-Moscow collaboration [30]. Interpreted in terms of a Majorana mass of the neutrino, this implies $m_{e e}$ between $0.12 \mathrm{eV}$ to $0.90 \mathrm{eV}$. If confirmed, this result is of fundamental significance. For a thorough discussions of this result (see also [31]) [32], we refer readers to the report of the double beta decay working group [8].

\subsubsection{Cosmology and neutrino masses}

A very different way to get information on the absolute scale of neutrino masses is from the study of the cosmic microwave radiation spectrum as well as the study of the large scale structure in the universe. A qualitative way of understanding why this is the case is that if neutrinos are present in abundance in the universe at the epoch of structure formation and have a sizable mass the formation of structure is affected. For instance, for a given neutrino mass $m$, all structure on a scale smaller than a certain value given by the inverse of neutrino mass is washed away by neutrino free-streaming. This implies a reduced power on smaller scales. Thus, accurate measurements of the galaxy power spectrum for small scales can help constrain or determine neutrino masses. Recent results from the WMAP and surveys of large scale structure have set a limit on the sum of neutrino masses $\sum m_{i} \leq 0.7-2 \mathrm{eV}[33,34]$. More recent results from the Sloan Digital Sky Survey (SDSS) place the limit of $\sum m_{i} \leq 1.6 \mathrm{eV}$. Hannestad [34] has emphasized that these upper limits can change if there are more neutrino species - e.g. for 5 neutrinos, $\sum m_{i} \leq 2.12 \mathrm{eV}$ if they are in equilibrium at the epoch of BBN.

A point worth emphasizing is that the above result is valid for both Majorana and Dirac neutrinos as long as the "right-handed" neutrinos decouple sufficiently earlier than the BBN epoch and are not regenerated subsequently ${ }^{3}$.

These limits already provide nontrivial information about neutrino masses: the limit $\sum_{i} m_{i}=0.7 \mathrm{eV}$, if taken at face value, implies that each individual neutrino mass is smaller than $0.23 \mathrm{eV}$, which is similar to the projected sensitivity of the proposed KATRIN experiment. PLANCK satellite observations are expected to be sensitive to even smaller

\footnotetext{
${ }^{3}$ In the Dirac case the "right-handed" degrees of freedom are decoupled because of the smallness of the corresponding Yukawa couplings. However, for very small temperatures, i.e. long after BBN, it is no longer appropriate to describe neutrinos in terms of chiral states. This means that strictly speaking there is a regeneration, but this does not affect BBN (see, e.g., [35]).
} 
values of $\sum_{i} m_{i}$, thereby providing a completely independent source of information on neutrino masses. These results may have implications for models of sterile neutrinos that attempt to explain the LSND results.

\subsubsection{CP violation}

It is clear from Eq. (11) that, for Majorana neutrinos, there are three CP-odd phases that characterize neutrino mixings [11,12], and our understanding of the leptonic sector will remain incomplete without knowledge of these $[36,37]$. There are two possible ways to explore CP phases: (i) one way is to perform long-baseline oscillation experiments and look for differences between neutrino and anti-neutrino survival probabilities [38]; (ii) another way is to use possible connections with cosmology. It has often been argued that neutrinoless double beta decay may also provide an alternative way to explore CP violation [39]. This is discussed in Sec. 3.

In summary, the most important goals of the next phase of neutrino oscillation experiments are:

(i) To determine the value of $\theta_{13}$ as precisely as possible;

(ii) To determine the sign of $\Delta m_{13}^{2}$, or the character of the neutrino mass hierarchy;

(iii) To improve the accuracy of the measurement of the other angles and the masssquared differences;

(iv) To probe the existence of the three CP odd phases as best as possible.

The discussion above assumes a minimal picture for massive neutrinos where the most general Majorana mass for three neutrinos has been added. While this may be the picture to the leading order, it is quite conceivable that there are other interesting subdominant effects that go beyond this. It is of utmost interest to determine to what extent one can constrain (or perhaps discover) these new nonstandard phenomena, since their absence up to a certain level (or, of course, their presence) will provide crucial insight into the detailed nature of the New Physics.

\subsubsection{Prospects for determining whether neutrinos are Majorana or Dirac}

As an example of what we can learn from future experiments, we focus on three experiments - searches for neutrinoless double beta decay (down to the level of $0.03 \mathrm{eV}$ level), studies to determine the sign of $\Delta m_{23}^{2} \equiv\left(m_{3}^{2}-m_{2}^{2}\right)$, and the KATRIN experiment, which is sensitive to the effects of a nonzero neutrino mass down to $0.2 \mathrm{eV}$ in tritium beta decay. The interplay between the possible results of these three experiments is summarized in Table 1

We see that extremely valuable information will follow from the results of these experiments.

\subsubsection{Sterile neutrinos}

A question of great importance in neutrino physics is the number of neutrino species. Measurement of the invisible $Z$-width in LEP-SLC experiments tell us that there are three types of light standard-model electroweak-doublet neutrinos that couple to the $W$ and $Z$ boson. These are the three known neutrinos $\nu_{e, \mu, \tau}$. This implies that if there are 
Table 1: Different possible conclusions regarding the nature of the neutrinos and their mass hierarchy from the three complementary experiments.

\begin{tabular}{|c||c||c||c|}
\hline$\beta \beta_{0 \nu}$ & $\Delta m_{13}^{2}$ & KATRIN & Conclusion \\
\hline yes & $>0$ & yes & Degenerate Hierarchy, Majorana \\
yes & $>0$ & no & Degenerate Hierarchy, Majorana or \\
& & & Normal Hierarchy, Majorana with heavy particle contribution \\
yes & $<0$ & no & Inverted Hierarchy, Majorana \\
yes & $<0$ & yes & Degenerate Hierarchy, Majorana \\
no & $>0$ & no & Normal Hierarchy, Dirac or Majorana \\
no & $<0$ & no & Dirac \\
no & $<0$ & yes & Dirac \\
no & $>0$ & yes & Dirac \\
\hline
\end{tabular}

other neutrino-like interaction eigenstates, then they must either be sufficiently massive that they cannot occur in the decay of the $Z$ or they must be electroweak singlets with no coupling to the $W$ or $Z$. In the latter case, the interaction eigenstates are called sterile neutrinos. In general, a neutrino mass eigenstate will be a linear combination of the three electroweak-doublet neutrinos and some unknown number of electroweak-singlet (= sterile) neutrinos. In the presence of electroweak-singlet neutrinos, the neutral weak current is not, in general, diagonal $[40,41]$. In common parlance, the word sterile neutrino is often used to denote a light electroweak-singlet neutrino and hence to exclude the heavy electroweak-singlet neutrino-like states that may well play a role in the seesaw mechanism. So the question is: are there any (light) sterile neutrinos and if so, how many are they and do they mix with the ordinary neutrinos?

Light sterile neutrinos have been postulated in order to explain [42] the data from the Los Alamos Liquid Scintillation Detector (LSND) experiment [43], where neutrino flavor conversion both from a stopped muon (DAR) as well as the one accompanying the muon in pion decay have apparently been observed. The evidence from the DAR is statistically more significant and is interpreted as an oscillation from $\bar{\nu}_{\mu}$ to $\bar{\nu}_{e}$. The mass and mixing parameter range that fits data is:

$$
\Delta m^{2} \simeq 0.2-2 \mathrm{eV}^{2}, \quad \sin ^{2} 2 \theta \simeq 0.003-0.03
$$

There are points at higher masses specifically at $6 \mathrm{eV}^{2}$ which are also allowed by the present LSND data for small mixings. The KARMEN experiment at the Rutherford laboratory has very strongly constrained the allowed parameter range of the LSND data [44]. Currently the MiniBooNE experiment at Fermilab is under way to probe the LSND parameter region [45].

Since this $\Delta m_{\mathrm{LSND}}^{2}$ is so different from that $\Delta m_{\odot, A}^{2}$, the simplest way to explain these results is to add one $[42,46]$ or two [47] sterile neutrinos. The sterile neutrinos raise important issues of consistency with cosmology as well as physics beyond the simple three neutrino picture and will be discussed in a subsequent section. 


\subsubsection{Neutrino electromagnetic dipole moments and neutrino decay}

A massive Dirac neutrino can have a diagonal magnetic (and a CP-violating electric) dipole moment. Because a Majorana neutrino is the same as its antiparticle, it has vanishing diagonal magnetic and electric dipole moments. A massive Dirac or Majorana neutrino can have nondiagonal, i.e., transition, magnetic and electric dipole moments. Some discussions of diagonal and transition neutrino electromagnetic moments in renormalizable electroweak gauge theories (where these can be calculated) include [48, 49], [40], [51]- [59]. In the standard model extended to contain massive Dirac neutrinos,

$\mu_{\nu_{j}}=3 e G_{F} m_{\nu_{j}} /\left(8 \pi^{2} \sqrt{2}\right)=1.6 \times 10^{-19}\left(m_{\nu_{j}} /(1 \mathrm{eV})\right) \mu_{B}$ for the neutrino mass eigenstate $\nu_{j}$ [52], where $\mu_{B}=\frac{e}{2 m_{e}}$ is a Bohr magneton. In left-right models and others with new physics beyond the standard model, this may be larger (e.g., [51,53, 57-59]). In contrast to the magnetic dipole moment, the neutrino electric dipole moment vanishes at one-loop order for a massive Dirac neutrino in the extended standard model [53]. However, in a left-right model, a Dirac neutrino may acquire an electric dipole moment at the one-loop level [53]. In the more generic case of a Majorana neutrino, one's interest focuses on the neutrino transition magnetic (and electric) dipole moments. The presence of these diagonal or transition moments allows for new electromagnetic interactions between neutrinos and other fermions of the Standard Model. In particular in neutrinoelectron scattering, in addition to the usual weak interaction contribution, there will be a photon exchange contribution to the scattering cross section. The existing neutrino scattering measurements therefore provide an upper limit on the neutrino magnetic moment: $\mu_{\nu_{e}} \leq(1-1.3) \times 10^{-10} \mu_{B}$. As we will discuss in more detail later, the observation of nonzero neutrino magnetic moment would be considered evidence of new physics at the $\mathrm{TeV}$ scale. The reason for that is that if all new physics is parameterized by (Majorana or Dirac) neutrino masses, or, equivalently, if all new physics effects are suppressed by the very large naive seesaw energy scale (close to the GUT scale) the neutrino magnetic moments are expected to be of order $10^{-19} \mu_{B}\left(\frac{m_{\nu}}{1 e V}\right)$. High-precision searches for a magnetic moment provide, therefore, complementary tools to probe the physics that is expected to lie just beyond the electroweak symmetry breaking scale.

A neutrino magnetic or electric dipole moment leads to new processes that can alter our understanding of energy balance in astrophysical systems such as in stars and supernovae [60]. It can also affect considerations involving the neutrinos in the early universe such as the BBN. In Sec. 6.1 we discuss more details on neutrino magnetic moments and what one can learn from various proposed experiments.

The existence of a neutrino magnetic or electric transition moment is also related to neutrino decays. For instance, it would allow heavier neutrinos to decay radiatively to the lighter ones $[40,48,50,54,61,62]$. Such decays can be detectable in astrophysical experiments. Present upper limits coupled with the general idea about spectra of neutrinos from oscillation experiments, imply that lifetimes of the primary mass eigenstates in electroweak-doublet neutrinos are larger than $10^{20}$ sec., much longer than the age of the universe $[40,48,50,54,61,62]$. Such decays do not therefore affect the evolution of the universe.

It is however possible that there are other scalar particles to which the neutrinos decay; one such example is the majoron, which is a Goldstone boson corresponding to the 
spontaneous breaking of a global $B-L$ symmetry [63]. The decay to these scalar bosons may occur at a faster rate [64] than that to photons and may therefore have astrophysical and cosmological implications [65]. This will be the subject of another working group [9]; so we only focus on the implications of the magnetic moment in one of the subsequent sections.

\subsection{Neutrino probes of other fundamental symmetries}

Neutrino experiments can also be used to probe the validity of other fundamental symmetries, some of which are often commonly assumed in theoretical discussions, as well as the basic assumptions of local quantum field theories on which the Standard Model is based. Some examples of these are:

- Violation of Lorentz invariance;

- CPT violation;

- Possible existence of new long range forces in nature associated with lepton number;

- Nonstandard interactions of neutrinos such as flavor changing neutral currents involving neutrinos.

We will explore to what extent existing limits on these departures from standard scenarios can be improved.

\subsection{Why neutrino mass necessarily means physics beyond the Standard Model?}

Neutrino oscillations are, to date, the only evidence for the existence of physics beyond the Standard Model (in the domain of particle physics). It is of utmost importance to decipher the kind of new physics indicated by the existing data and to anticipate the signals of new physics that might appear in future planned observations. We must understand how and if they fit into the different big pictures that have been advocated for independent reasons, including the gauge hierarchy problem and gauge coupling unification. To discuss this, we first introduce the Standard Model and possible ways to extend it to accommodate the neutrino observations.

In the Standard Model, which is based on the gauge group $S U(3)_{c} \times S U(2)_{L} \times U(1)_{Y}$, the quarks and leptons transform as $Q_{L}\left(3,2, \frac{1}{3}\right), u_{R}\left(3,1, \frac{4}{3}\right), d_{R}\left(3,1,-\frac{2}{3}\right), L(1,2,-1)$, $e_{R}(1,1,-2)$. The Higgs boson $H$, responsible for electroweak symmetry breaking, transforms as $(1,2,+1)$. The electroweak symmetry $S U(2)_{L} \times U(1)_{Y}$ is broken by the vacuum expectation of the Higgs doublet $\left\langle H^{0}\right\rangle=v_{\mathrm{wk}} \simeq 246 \mathrm{GeV}$, which renders the $W^{ \pm}$and $Z^{0}$ gauge bosons and the electrically charged fermions massive. The reason neutrinos do not get mass as a result of the Higgs mechanism is that the right-handed neutrino $N_{R}$ was not included in the list of fermions in the Standard Model; as a result there is no coupling of the form $h_{\nu} \bar{L} H N_{R}$ that could have given mass to the neutrinos after symmetry breaking.

One seemingly straightforward way to understand the neutrino mass would be to extend the Standard Model to include the $N_{R}$. This would also be desirable from the 
point of view of making the model quark lepton symmetric. There are two problems with this naïvely trivial modification. One is that by quark lepton symmetry one would expect the neutrino masses arising from the Yukawa coupling $h_{\nu} \bar{L} H N_{R}$ to be of the same order as the quark and charged leptons. Observations suggest that neutrino masses are at least $10^{6}$ times smaller than the smallest quark and lepton masses. Therefore, a nonzero neutrino mass not only suggests the existence of right-handed neutrinos (of which there would be three if they correspond to the usual generations), but some new physics that will enable us to understand why $M_{\nu} \ll m_{q, \ell}$. The seesaw mechanism provides a plausible basis for this understanding, since it makes use of the fact that, among the known fermions, only neutrinos can have Majorana mass terms. Thus, ironically, we may have a better way to understand the lightness of the neutrinos than we do to understand the generational hierarchy factor of $\sim 10^{6}$ between the masses of the top quark and the electron, for which there is no accepted explanation at present.

The other problem with introducing a set of right-handed neutrino fields is the fact that they are Standard Model gauge singlets. This means that, as far as the symmetries of the Standard Model are concerned, a Majorana mass for the $N_{R}$ fields is allowed. If such a mass term is present, however, the neutrino masses are not simply given by the $h_{\nu} v_{\mathrm{wk}}$, but are determined by a more complicated function of $h_{\nu} v_{\mathrm{wk}}$ and the Majorana masses of the right-handed neutrinos. In order to avoid the presence of a Majorana mass for the righthanded neutrinos one is required to impose an extra symmetry to the Standard Model Lagrangian (say, lepton number) - a very nontrivial modification of what is traditionally referred to as the Standard Model of electroweak interactions.

\subsubsection{Seesaw mechanism for small neutrino masses}

A simple way to understand the smallness of neutrino mass within this minimally extended Standard Model is to break lepton number symmetry (or $B-L$ symmetry) and add a Majorana mass for the right-handed neutrino $M_{R} N_{R}^{T} C^{-1} N_{R}$. Thus the two terms that give mass to the neutrinos have the form $h_{\nu} v_{\mathrm{wk}} \bar{\nu}_{L} N_{R}+M_{R} N_{R}^{T} C^{-1} N_{R}+$ h.c. Assuming $n$ "left-handed" neutrinos $\nu_{L}$ and $m$ "right-handed neutrinos $N_{R}$, the $(n+m) \times(n+m)$ Majorana neutrino mass matrix is

$$
\mathcal{M}=\left(\begin{array}{cc}
0 & h_{\nu} v_{\mathrm{wk}} \\
h_{\nu} v_{\mathrm{wk}} & M_{R}
\end{array}\right) .
$$

In the limit $M_{R} \gg h v_{\mathrm{wk}}$, the eigenvalues of this matrix are given by $-\frac{\left(h_{\nu} v_{\mathrm{wk}}\right)^{2}}{M_{R}}$ and $M_{R}$, with respective approximate eigenvectors $\nu_{L}$ and $N_{R}$. The effective active neutrino masses are clearly much smaller than typical charged fermion masses (which are of order $h_{\nu} v_{\mathrm{wk}}$ ) as long $M_{R} \gg v_{\mathrm{wk}}$. This is the well-known seesaw mechanism [66-70]. If we take as a guide a value for $h_{\nu} \leq 1$, then atmospheric neutrino data requires that $M_{R} \leq 10^{15} \mathrm{GeV}$. It should be emphasized that there is very little concrete information or experimental guidance regarding the magnitude of $M_{R}$, which is virtually unconstrained [71]. One question which arises is why this value rather than $M_{\mathrm{Pl}} \simeq 10^{18} \mathrm{GeV}$, which, one may argue, would have been a more natural value? Could this be an indication of a new symmetry? The answer to this question is obviously of fundamental significance. 
An example of such a symmetry is the $B-L$ symmetry embodied in the left-right symmetric models based on the gauge group $S U(2)_{L} \times S U(2)_{R} \times U(1)_{B-L}$ [72]. This gauge group is also a subgroup of $S O(10)$ grand unification group. The above mentioned value of $M_{R}$ is rather close to the conventional GUT scale of $10^{16} \mathrm{GeV}$. This makes the seesaw mechanism a very attractive framework for discussing the neutrino mass. We will discuss further consequences of grand unification for neutrino masses in a subsequent section. We will also explore in this review unification-model independent consequences of the seesaw mechanism.

\subsubsection{Type I vs type II seesaw mechanism}

If there are indeed right-handed neutrinos, the most general Majorana mass matrix that mixes active and sterile neutrinos is given by Eq. (3) with the $n \times n$ zero matrix in the upper left-hand corner replaced by a generic (symmetric) matrix $M_{L}$. This phenomenon occurs, for example, when the theory containing the $N_{R}$ becomes parity symmetric as is the case for $S U(2)_{L} \times S U(2)_{R} \times U(1)_{B-L}$ or $S O(10)$ based models. In this case the seesaw formula is modified to

$$
M_{\nu}^{\mathrm{II}}=M_{L}-M_{\nu}^{D} M_{R}^{-1}\left(M_{\nu}^{D}\right)^{T},
$$

where, in an $S U(2)_{L} \times S U(2)_{R} \times U(1)_{B-L}$ symmetric model, $M_{L}=f v_{L}$ and $M_{R}=f v_{R}$, where $v_{L, R}$ are the vacuum expectation values of Higgs fields that couple to the right and left-handed neutrinos. Eq. (44) is called the type II seesaw relation [73]. It should be noted that in the absence of a discrete left-right symmetry, $M_{L}$ is in general not related to $M_{R}$.

\subsubsection{Triplet seesaw}

An alternative way to understand the small neutrino mass without introducing the right handed neutrino is the triplet seesaw mechanism. It was pointed out in various papers [74] in early 1980 that if the standard model is extended by the addition of a triplet Higgs $\Delta_{L}$ with weak hypercharge $Y=2$, a vev for it can lead to Majorana mass for the neutrinos from the interaction $f_{\nu} \psi_{L}^{T} C^{-1} \tau_{2} \vec{\tau} \cdot \vec{\Delta} \psi_{L}$ ( $\psi_{L}$ being the lepton doublet $\left(\nu_{L}, e_{L}\right)$ ). However, one has to tune the Yukawa coupling $f_{\nu}$ by about $10^{-10}$ or so to get desirable neutrino masses. It has subsequently been shown [75] that in the context of grand unified theories, the triplet vev is given by the formula $\left\langle\Delta_{L}^{0}>\sim \frac{v_{w k}^{2}}{M_{U}}\right.$, where $M_{U}$ is close to the grand unification scale and corresponds to the physical mass of the triplet Higgs field. Since $M_{U} \gg v_{w k}$, this provides a natural suppression of the triplet vev and the right order for the neutrino mass emerges. Note also that this can also emerge from the type II seesaw formula in the limit of $M_{N_{R}} \rightarrow \infty$. In this case, the neutrino mass matrix is directly proportional to the coupling matrix $f_{\nu}$.

\subsubsection{Seesaw with triplet fermions}

Yet another possible extension of the standard model without right handed neutrinos which leads to small neutrino masses is to postulate the existence of triplet vectorlike fermions: $\vec{\Lambda}[76]$. Since a vectorlike triplet can have an arbitrary mass, it also leads to seesaw mass formula. 


\subsubsection{Understanding large mixings within the seesaw mechanism}

A major puzzle of quark-lepton physics is the fact that the quark mixing matrix and the leptonic one are qualitatively different. In order to understand the mixing angles [4,77], we have to study the mass matrices for the charged leptons and neutrinos.

A general approach

To see the possible origin of neutrino mixings, one can start with the following form for the mass part of the neutrino Lagrangian:

$$
\mathcal{L}_{\text {mass }}=\bar{\nu}_{L} M_{\nu}^{D} N_{R}+\bar{e}_{L} M_{\ell} e_{R}+N_{R}^{T} M_{R} N_{R}+\text { h.c. }
$$

Using the seesaw mechanism one can derive from this equation, the formula for neutrino masses can be written as for the case of type I seesaw:

$$
M_{\nu}^{\mathrm{I}}=-M_{\nu}^{D} M_{R}^{-1}\left(M_{\nu}^{D}\right)^{T},
$$

To obtain the lepton mixing matrix, one can diagonalize the charged lepton mass matrix by $M_{\ell}=U_{\ell} M_{\ell}^{d} V^{\dagger}$ and $m_{\nu}=\left(U^{*}\right)_{\nu} m_{\nu}^{d}\left(U^{\dagger}\right)_{\nu}$ and find that $U=U_{\ell}^{\dagger} U_{\nu}$.

With this theoretical preamble, understanding of neutrino mixings can proceed along two paths. In theories where quark and lepton mixings are disconnected (such as many weak scale theories), one may like to pass to a basis where the charged lepton masses are diagonal. In that case, all the neutrino mixing information is in the effective neutrino mass matrix. One can then look for the types of mass matrices for neutrinos that can lead to bi-large mixings and try to understand them in terms of new physics. Here we give a brief overview of some generic structures for $M_{\nu}$ that do the job.

(i) The case of normal hierarchy: In this case, one neutrino mass matrix that leads to "bi-large" mixing has the form:

$$
M_{\nu}=m_{0}\left(\begin{array}{ccc}
\epsilon & \epsilon & \epsilon \\
\epsilon & 1+\epsilon & 1 \\
\epsilon & 1 & 1
\end{array}\right)
$$

where $m_{0}$ is $\sqrt{\Delta m_{\mathrm{A}}^{2}}$. We have omitted order one coefficients in front of the $\epsilon$ 's. This matrix leads to $\tan \theta_{A} \simeq 1, \Delta m_{\odot}^{2} / \Delta m_{\mathrm{A}}^{2} \simeq \epsilon^{2}$ and also to a large solar angle. For the LMA solution, we find the interesting result that $\epsilon \sim \lambda$ where $\lambda$ is the Cabibbo angle $(\simeq 0.22)$. This could be a signal of hidden quark lepton connection ${ }^{4}$. In fact we will see below that in the context of a minimal $S O(10)$ model, this connection is realized in a natural manner. (ii) The case of inverted hierarchy: The elements of the neutrino mass matrix in this case have a pattern

$$
m_{\nu}=m_{0}\left(\begin{array}{ccc}
\epsilon & c & s \\
c & \epsilon & \epsilon \\
s & \epsilon & \epsilon
\end{array}\right) .
$$

where $c=\cos \theta$ and $s=\sin \theta$ and it denotes the atmospheric neutrino mixing angle. An interesting point about this mass matrix is that in the limit of $\epsilon \rightarrow 0$, it possesses

\footnotetext{
${ }^{4}$ Alternatively, the relation $\theta_{\odot}+\lambda=\pi / 4$, nowadays known as quark-lepton-complementarity, can also be interpreted as such a connection [78].
} 
an $L_{e}-L_{\mu}-L_{\tau}$ symmetry [79]. One therefore might hope that if inverted hierarchy structure is confirmed, it may provide evidence for this leptonic symmetry. This can be an important clue to new physics beyond the Standard Model. This issue of leptonic symmetries will be discussed in the main body of this report.

(iii) Degenerate neutrinos: One may either add a unit matrix to the just mentioned mass matrices and look for new physics models for them; alternatively, one may look for some dynamical ways by which large mixings can arise. It turns out that if neutrinos are mass degenerate, one can generate large mixings out of small mixings [80-84] purely as a consequence of radiative corrections. We will call this possibility radiative magnification and will discuss it in a future section.

In grand unified theories, quark and lepton mass matrices are connected. One may therefore lose crucial information about symmetries if one works in a basis where the charged leptons are diagonal. Furthermore, if either of the quark (up or down) mass matrices are chosen diagonal, it may not even be possible to go to the diagonal charged lepton basis. Thus in this case, we have $U=U_{\ell}^{\dagger} U_{\nu}$. So one may seek an understanding of large mixings in the charged lepton sector. For example in $S U(5)$ type theories, $U_{\ell}$ is related to the mixings of right-handed quarks which are unobservable in low energy weak interactions and can therefore be the source of large mixings. Models of this type are called lopsided mixing models [85].

The basic strategy then would be to look for clues for new symmetries in the structure of the mass matrices, which could then provide information about the nature of physics beyond the Standard Model. The symmetries of course may become obscured by our choice of basis where the charged leptons are diagonal. It is this which gives different possibilities for arriving at the bi-large mixings and the hope is that different strategies will lead to different predictions for observables, which can then be put to experimental test.

\subsubsection{Alternatives to high-scale seesaw}

While the high-scale seesaw mechanism is the simplest and perhaps the most elegant way to understand the small neutrino masses and become couched in a quark leptonic and parity symmetric framework leading to simple grand unification theories, there are alternatives to seesaw which can also explain the small neutrino masses [86-95]. In such a case the neutrinos can either be Dirac or Majorana fermions depending on the theory.

Unlike the non-supersymmetric seesaw models, alternatives such as the one presented in [90] often predict observable charged lepton lepton-flavor violating signals [96], e.g., $\mu \rightarrow e \gamma, \mu, \tau \rightarrow e e e$, etc. More generically, searches for charged-lepton flavor violation crucially help distinguish among the several theoretical interpretations of the origin of neutrino masses.

\subsection{Summary of the Introduction}

Some of the questions that we would like to answers in the course of this work are:

- Can we decide whether the neutrino is a Dirac or Majorana particle? 
- To what extent can the planned neutrino experiments pin down the structure of the three neutrino mass matrix? This involves such questions as determining the sign of $\Delta m_{23}^{2}$, higher precision measurement of mixing parameters, etc.

- What is the impact of a $\theta_{13}$ measurement (and the improved determination of the other elements of the lepton mixing matrix) on the general landscape of physics beyond the Standard Model? We find that $\theta_{13}$ is a powerful discriminator of models.

- Can we test the seesaw hypothesis and discriminate between different types of seesaw using lepton flavor violation and other "non-neutrino probes?"

- How can one experimentally discover or limit physics beyond the standard scenario? This will address such aspects as:

(1) Flavor changing neutral currents for neutrinos; present limits and future prospects;

(2) Admixtures of sterile neutrinos, both heavy and light;

(3) magnetic moments of neutrinos.

- What can we learn about CP violation in the lepton sector and how can we connect it to the question of the origin of matter via leptogenesis. Given what we know about the neutrino masses, assuming thermal leptogenesis, do we have an explanation of the observed baryon to photon ratio? 


\section{What can we learn about neutrino mass matrices from experiments?}

In this section we briefly review our ability to reconstruct the neutrino mass matrix. We will also discuss (from "the bottom up") what we hope to learn from the neutrino mass matrix itself, instead of trying to quantify what different models predict for the neutrino mass matrix. See, for example, $[4,77]$ for reviews of a few different models. In a subsequent section, we will discuss the connection of neutrino masses to GUTs, and will spend a little more time on "top-down" predictions for neutrino masses and mixing angles.

As mentioned earlier, we will assume that the neutrinos are Majorana fermions. While there is no experimental evidence that this is the case, the majority of the theoretical HEP community considers it more likely that the neutrinos are Majorana fermions, and a larger amount of phenomenological research effort has gone into understanding and interpreting Majorana neutrino mass matrices than Dirac mass matrices. For some discussions of Dirac neutrino mass matrices and how they are related to the large mixing in the leptonic sector and the neutrino mass-squared differences, see, for example, [97].

Below the electroweak phase transition, the Majorana neutrino mass matrix $m_{\nu}$ is the coefficient of the operator (using four-component-spinor notation)

$$
\frac{1}{2} m_{\nu}^{\alpha \beta} \nu_{\alpha}^{T} C^{-1} \nu_{\beta}+H . c .
$$

where $\alpha, \beta=e, \mu, \tau, \ldots$ are flavor indices, and $m_{\nu}^{\alpha \beta}$ are the components of the neutrino

mass matrix (note that $m_{\nu}$ is symmetric, i.e., $m_{\nu}^{\alpha \beta}=m_{\nu}^{\beta \alpha}$ ). In this section we will concentrate on a purely active $3 \times 3$ mass matrix. A detailed discussion of $4 \times 4$ (and larger) mass matrices, which also allow for the existence of fourth generation and/or sterile neutrinos is the subject of subsequent sections. Note that Eq. (9) is not sensitive to the mechanism that generates neutrino masses. These will be discussed in more detail in a later section.

In general, one cannot work back from a knowledge of the observed lepton mixing matrix to the individual nondiagonal mass matrices in the charged lepton and neutrino sectors. It is, indeed, the diagonalization of both of these mass matrices that gives rise to the observed lepton mixing, and models exist where the mixing in the charged lepton sector is large. One can always choose to work on the weak basis where the charged lepton mass matrix is diagonal - the price one pays for doing this is that the flavor structure of the theory may not be manifest. In this case, one can calculate the neutrino mass matrix in terms of the observed lepton mixing matrix as

$$
m_{\nu}^{\alpha \beta}=\sum_{i}\left(U^{*}\right)_{\alpha i} m_{i}\left(U^{\dagger}\right)_{i \beta}
$$

We choose sign conventions such that the neutrino mass eigenvalues are real and positive. By choosing to write $U=V K$, where $V$ and $K$ are given by Eq. (1) we have removed all of the redundancy contained in $m_{\nu}$ associated with re-defining the neutrino fields by a complex phase. Hence, $m_{\nu}$ as defined by Eq. (10) is only a function of observable parameters. The phases in $K$ are the so-called Majorana phases [11,12]. They can be 
redefined away by allowing the neutrino mass eigenvalues to be complex. In this case, $U=V, m_{1}$ is real and positive, and $m_{2}=\left|m_{2}\right| e^{-2 i \phi_{1}}, m_{3}=\left|m_{3}\right| e^{-2 i \phi_{2}}$.

In the near future, we hope to significantly improve the determination of the elements of the neutrino mass matrix, although some uncertainty will still remain (for a detailed discussion, see, for example, [98]). Through neutrino oscillation experiments, all three mixing angles $\theta_{12}, \theta_{23}$, and $\theta_{13}$ are expected to be determined with good precision (this is one of the main goals of next-generation neutrino oscillation experiments, discussed in great lengths in this report), while there is hope that the "Dirac phase" $\delta$ can be probed via long-baseline $\nu_{\mu} \rightarrow \nu_{e}$ oscillation searches. Neutrino oscillation experiments will also determine with good precision the neutrino mass-squared differences $\left(\Delta m_{12}^{2}\right.$ at the $5 \%-$ $10 \%$ level, $\Delta m_{13}^{2}$ [including the sign] at the few percent level). In order to complete the picture, three other quantities must also be measured, none of which is directly related to neutrino oscillations.

One is the overall scale for neutrino masses. As already briefly discussed, this will be probed, according to our current understanding, by studies of the end-point spectrum of beta-decay, searches for neutrinoless double beta decay, and cosmological observations (especially studies of large-scale structure formation). Note that neutrinoless double beta decay experiments are sensitive to $\left|m_{\nu}^{e e}\right|$, i.e., they directly measure the absolute value of an element of $m_{\nu}$.

The other two remaining observables are the "Majorana" phases. Neutrinoless double beta decay experiments are sensitive to a particular combination of these, the so-called effective Majorana mass,

$$
\left|m_{\nu}^{e e}\right| \equiv\langle m\rangle_{e f f}=\left|\cos ^{2} \theta_{13}\left(\left|m_{1}\right| \cos ^{2} \theta_{12}+\left|m_{2}\right| e^{2 i \phi_{1}} \sin ^{2} \theta_{12}\right)+\sin ^{2} \theta_{13}\right| m_{3}\left|e^{2 i \phi_{2}}\right| .
$$

With present uncertainties in the nuclear matrix elements, however, it seems at least very challenging [99] to obtain any information regarding Majorana phases from neutrinoless double beta decay. For a detailed study, see, for example, [100].

A few comments are in order. First, the relation between the rate for neutrinoless double beta decay and the Majorana phases and neutrino masses only holds under the assumption that the neutrino masses are the only source of lepton-number violation (as far as neutrinoless double beta decay is concerned). Second, only one or a combination of the two independent Majorana phases can be determined in this way. It is fair to say that there is no realistic measurement one can look forward to making in the near future that will add any information and help us disentangle the "other" Majorana phase. Third, it is curious to note that the effect the Majorana phases have on the rate for neutrinoless double beta decay is CP even [37]. While Majorana phases can mediate CP violating phenomena [37], it seems unlikely that any of them can be realistically studied experimentally in the foreseeable future. For further discussion of CP violation among neutrinos see Ref. [101].

In spite of all the uncertainty due to our inability to measure Majorana phases, it is fair to say that we expect to correctly reconstruct several features of the neutrino mass matrix [98], especially if the overall mass scale and the neutrino mass hierarchy are determined experimentally. What do we hope to accomplish by reconstructing the neutrino mass matrix? The answer is that we wish to uncover whether there are new fundamental organizing principles responsible for explaining in a more satisfying way 
the values of the neutrino masses and the leptonic mixing angles. In other words, we would like to establish whether there is a fundamental reason behind the fact that the $\nu_{3}$ state contains almost the same amount of $\nu_{\mu}$ and $\nu_{\tau}$, while at the same time containing a relatively small amount of $\nu_{e}$. Are there flavor (or family) symmetries, capable of dynamically distinguishing the different generations of quarks and leptons and, we hope, explaining why there are three quasi-identical particles for each matter field?

In the neutrino sector, we are only getting started. We have, for example, identified several textures for the neutrino mass matrix that lead to the currently observed masssquared differences and mixing angles, and have identified some of the measurements that will allow us to identify which textures best describe Nature. As has been already pointed out, it is not clear whether this is the best avenue to pursue as far as identifying whether there is a deep explanation for the patterns we observe in experiments. For example, it may turn out that we have made a weak-basis choice that renders the job more complicated (it is possible that the mixing angles are "determined by the charged lepton sector" [102-104]), or that all the structure contained in the neutrino sector is obscured after heavy degrees of freedom are integrated out (as may happen in type-I seesaw models). Nonetheless, we will discuss a few of these textures in order to exemplify some of the measurements (and how precise they should be) that will shed a significant amount of light in the issue of interpreting neutrino masses and mixing angles.

Arguably the simplest assumption one can make is that there is no symmetry or dynamical principle that explains why leptonic mixing angles are large [105]. This "flavorless" neutrino flavor model is often referred to as "neutrino mass anarchy" and is, currently, compatible with data $[105,106]$. Curiously, the anarchical hypothesis is not without predictions: it requires that the unobserved magnitude of the $U_{e 3}$ element of the leptonic mixing matrix is $\left|U_{e 3}\right|^{2}>0.01$ at the $95 \%$ confidence level (see [106] for details and a proper definition of this bound). This means that after the next-generation of reactor and/or long-baseline experiments analyze their data we will know whether we can afford a "random" leptonic mixing matrix or not. It should be noted that this model applies only for the leptonic mixing matrix - it has nothing specific to say about the order of magnitude of neutral and charged lepton masses, or their hierarchies.

If one assumes that there is a nontrivial texture to the neutrino mass matrix, and that this texture "explains" the observed values of the mixing parameters, there are several completely different options. Some are tabulated in Table 2, and will be discussed briefly. Before proceeding, however, it is important to explain how these textures should be interpreted. The hypothesis is that, at leading order, the neutrino mass matrix can be parametrized by far fewer than the usual six complex coefficients. These are chosen in such a way that the dominant features of neutrino masses and mixings are explained. These are: (i) $m_{3}^{2}$ is either much larger or much smaller than $m_{1}^{2}, m_{2}^{2}$. This splitting determines the atmospheric mass-squared difference. (ii) the $\nu_{e}$ content of the $\nu_{3}$ state is zero. (iii) the $\nu_{\mu}$ and $\nu_{\tau}$ contents of the $\nu_{3}$ state are similar (or, perhaps, identical). In order to accommodate the other observed features (like $\left|U_{e 3}\right|$, the solar mass-squared difference and the solar angle) one includes sub-leading effects that violate the leadingorder structure. The structure of these sub-leading effects determines the "predictions" for the observables that are not determined by the leading order mass-texture. In Table 2, we list the predictions obtained in the case of a structureless sub-leading mass matrix, i.e., 


\begin{tabular}{|c|c|c|c|c|c|c|}
\hline Case & Texture & Hierarchy & $\left|U_{e 3}\right|$ & $\begin{array}{c}\left|\cos 2 \theta_{23}\right| \\
\text { (n.s.) }\end{array}$ & $\left|\cos 2 \theta_{23}\right|$ & Solar Angle \\
\hline A & $\frac{\sqrt{\Delta m_{13}^{2}}}{2}\left(\begin{array}{ccc}0 & 0 & 0 \\
0 & 1 & 1 \\
0 & 1 & 1\end{array}\right)$ & Normal & $\sqrt{\frac{\Delta m_{12}^{2}}{\Delta m_{13}^{2}}}$ & $\mathcal{O}(1)$ & $\sqrt{\frac{\Delta m_{12}^{2}}{\Delta m_{13}^{2}}}$ & $\mathcal{O}(1)$ \\
\hline B & $\sqrt{\Delta m_{13}^{2}}\left(\begin{array}{ccc}1 & 0 & 0 \\
0 & \frac{1}{2} & -\frac{1}{2} \\
0 & -\frac{1}{2} & \frac{1}{2}\end{array}\right)$ & Inverted & $\frac{\Delta m_{12}^{2}}{\left|\Delta m_{13}^{2}\right|}$ & - & $\frac{\Delta m_{12}^{2}}{\left|\Delta m_{13}^{2}\right|}$ & $\mathcal{O}(1)$ \\
\hline C & $\frac{\sqrt{\Delta m_{13}^{2}}}{\sqrt{2}}\left(\begin{array}{ccc}0 & 1 & 1 \\
1 & 0 & 0 \\
1 & 0 & 0\end{array}\right)$ & Inverted & $\frac{\Delta m_{12}^{2}}{\left|\Delta m_{13}^{2}\right|}$ & $\mathcal{O}(1)$ & $\frac{\Delta m_{12}^{2}}{\left|\Delta m_{13}^{2}\right|}$ & $\begin{array}{c}\left|\cos 2 \theta_{12}\right| \\
\sim \frac{\Delta m_{12}^{2}}{\left|\Delta m_{13}\right|}\end{array}$ \\
\hline Anarchy & $\sqrt{\Delta m_{13}^{2}}\left(\begin{array}{ccc}1 & 1 & 1 \\
1 & 1 & 1 \\
1 & 1 & 1\end{array}\right)$ & Normal & $>0.1$ & $\mathcal{O}(1)$ & - & $\mathcal{O}(1)$ \\
\hline
\end{tabular}

Table 2: Different leading-order neutrino mass textures and their "predictions" for various observables. The fifth column indicates the "prediction" for $\left|\cos 2 \theta_{23}\right|$ when there is no symmetry relating the different order one entries of the leading-order texture ('n.s.' stands for 'no structure', meaning that the entries of the matrices in the second column should all be multiplied by and order one coefficient), while the sixth column indicates the "prediction" for $\left|\cos 2 \theta_{23}\right|$ when the coefficients of the leading order texture are indeed related as prescribed by the matrix contained in the second column. See text for details. One may argue that the anarchical texture prefers but does not require a normal mass hierarchy.

one proportional to the anarchical texture [107]. In the case of a structured sub-leading mass matrix, expectations may vary significantly from these quoted in Table 2.

Case A is characterized by large entries in the " $\mu-\tau$ " sub-matrix, and small entries in the " $e$ " column and row. The determinant of the " $\mu-\tau$ " sub-matrix is constrained to be small in order to guarantee a hierarchy between the two independent mass-squared differences. The hierarchy of the neutrino masses is predicted to be normal $\left(m_{3}^{2}>m_{2}^{2}>\right.$ $m_{1}^{2}$ ). Maximal atmospheric mixing can be imposed at the leading order by requiring that the " $\mu-\tau$ " sub-matrix is democratic. The introduction of sub-leading effects leads to a "large" $\left|U_{e 3}\right|$ and $\cos 2 \theta_{23}$, of order the square-root of the ratio of mass-squared differences, which is $\mathcal{O}(0.1)$. If this texture is indeed realized in nature, we expect to observe a nonzero $\left|U_{e 3}\right|$ and a deviation of the atmospheric mixing from maximal at next-generation experiments. It may prove difficult to distinguish between case $\mathrm{A}$ and the anarchical texture via neutrino oscillation measurements alone. One potential discriminant seems to be the expected rate for neutrinoless double beta decay.

Case B is characterized by small " $e-\mu$ " and " $e-\tau$ " entries, a small determinant of the " $\mu-\tau$ " submatrix, and the constraint that the trace of $m_{\nu}$ is close to $2 m_{\nu}^{e e}$. In this case, one predicts an inverted mass hierarchy $\left(m_{2}^{2}>m_{1}^{2} \gg m_{3}^{2}\right)$, and both $\left|U_{e 3}\right|$ and $\cos 2 \theta_{23}$ are constrained to be of order the ratio of the mass-squared differences $(\mathcal{O}(0.01))$. The system is constrained enough that it is hard to obtain a much larger deviation of the atmospheric angle from maximal or a much larger $\left|U_{e 3}\right|$, while much smaller ones are, of course, obtainable if the sub-leading contributions are structured. If case B is indeed realized in Nature, there is a good chance that no $\left|U_{e 3}\right|$ effects will be observed in 
next-generation oscillation experiments, while precise measurements of the atmospheric mixing angle will remain consistent with $\theta_{23}=\pi / 4$ (equivalently, if a large deviation of the atmospheric angle is detected this texture will be ruled out). On a more positive note, one should expect a "large" rate for neutrinoless double beta decay $\left(m_{\nu}^{e e} \sim \sqrt{\Delta m_{13}^{2}}\right)$. A texture which is naïvely similar to case B is to change the sign of the " $\mu-\tau$ " sub-matrix, such that the trace of the leading order mass matrix is close to zero. This case, however, is disfavored by solar data, as the solar angle is constrained to be too small (for a more detailed discussion see, for example, [107]).

Case $\mathrm{C}$ is characterized by " $e-\mu$ " and " $e-\tau$ " entries which are much larger than all the other ones (set to zero at leading order) and thus corresponds to the case of approximate $L_{e}-L_{\mu}-L_{\tau}$ symmetry [79]. It leads to an inverted mass hierarchy, and a close to bimaximal [108] leading order mixing matrix. The solar angle is (at leading order) exactly maximal, while the atmospheric angle is generically large, becoming maximal in the limit when $m_{\nu}^{e \mu}= \pm m_{\nu}^{e \tau}$ (for real $m_{\nu}^{e \alpha}$ ). Sub-leading corrections to case $\mathrm{C}$ which are responsible for splitting the two heavy leading-order mass eigenstates will induce a $\left|U_{e 3}\right|$, $\cos 2 \theta_{23}$ and $\cos 2 \theta_{12}$ of order the ratio of the mass-squared differences $(\mathcal{O}(0.01))$, or smaller. Hence, similar to case $\mathrm{B}$, it seems unlikely that $U_{e 3}$-effects will be measured at next-generation experiments. This scenario is currently disfavored, as it also predicts a solar angle $\theta_{12}$ very close to $\pi / 4[102,107,109]$. One should not conclude, however, that scenarios based on "perturbations" around bi-maximal mixing are ruled out. A related issue has been discussed in detail recently by the authors of $[102,103]$.

A realistic three generation extension of the mass matrix in case (A) that leads to large but not maximal solar neutrino mixing is given by

$$
M_{\nu}=\frac{\sqrt{\Delta m_{A}^{2}}}{2}\left(\begin{array}{ccc}
c \epsilon^{n} & b \epsilon & d \epsilon \\
b \epsilon & 1+c \epsilon & -1 \\
d \epsilon & -1 & 1+\epsilon
\end{array}\right)
$$

Note that if $b=d$ and $c=1$, the atmospheric neutrino mixing is maximal and the mixing parameter $\theta_{13}=0$ [110]. In this case the mass matrix has $\mu-\tau$ interchange symmetry. Depending on how this symmetry is broken, the parameter $\theta_{13}$ is either of order $\sqrt{\frac{\Delta m_{\odot}^{2}}{\Delta m_{A}^{2}}}$ (for $c \neq 1$ ) or $\frac{\Delta m_{\odot}^{2}}{\Delta m_{A}^{2}}($ for $b \neq d)$ [111]. Therefore search for $\theta_{13}$ down to the level of 0.01 will be a big help in determining the structure of the neutrino mass matrix for the case of normal hierarchy. In both these cases, there is an important correlation between $\theta_{13}$ and $\theta_{A}-\frac{\pi}{4}[111]$.

In general, a neutrino mixing matrix originating from a $\mu-\tau$ symmetric mass matrix has the following structure (for simplicity, we did not include here the Majorana phases)

$$
U=\left(\begin{array}{ccc}
\cos \theta_{12} & \sin \theta_{12} & 0 \\
-\frac{\sin \theta_{12}}{\sqrt{2}} & \frac{\cos \theta_{12}}{\sqrt{2}} & \frac{1}{\sqrt{2}} \\
\frac{\sin \theta_{12}}{\sqrt{2}} & -\frac{\cos \theta_{12}}{\sqrt{2}} & \frac{1}{\sqrt{2}}
\end{array}\right)
$$

Note that the mass spectrum of the neutrinos is not predicted by the $\mu-\tau$ symmetry. Depending on the value of $\theta_{12}$, several interesting mixing schemes can arise: if $\theta_{12}=\pi / 4$ 


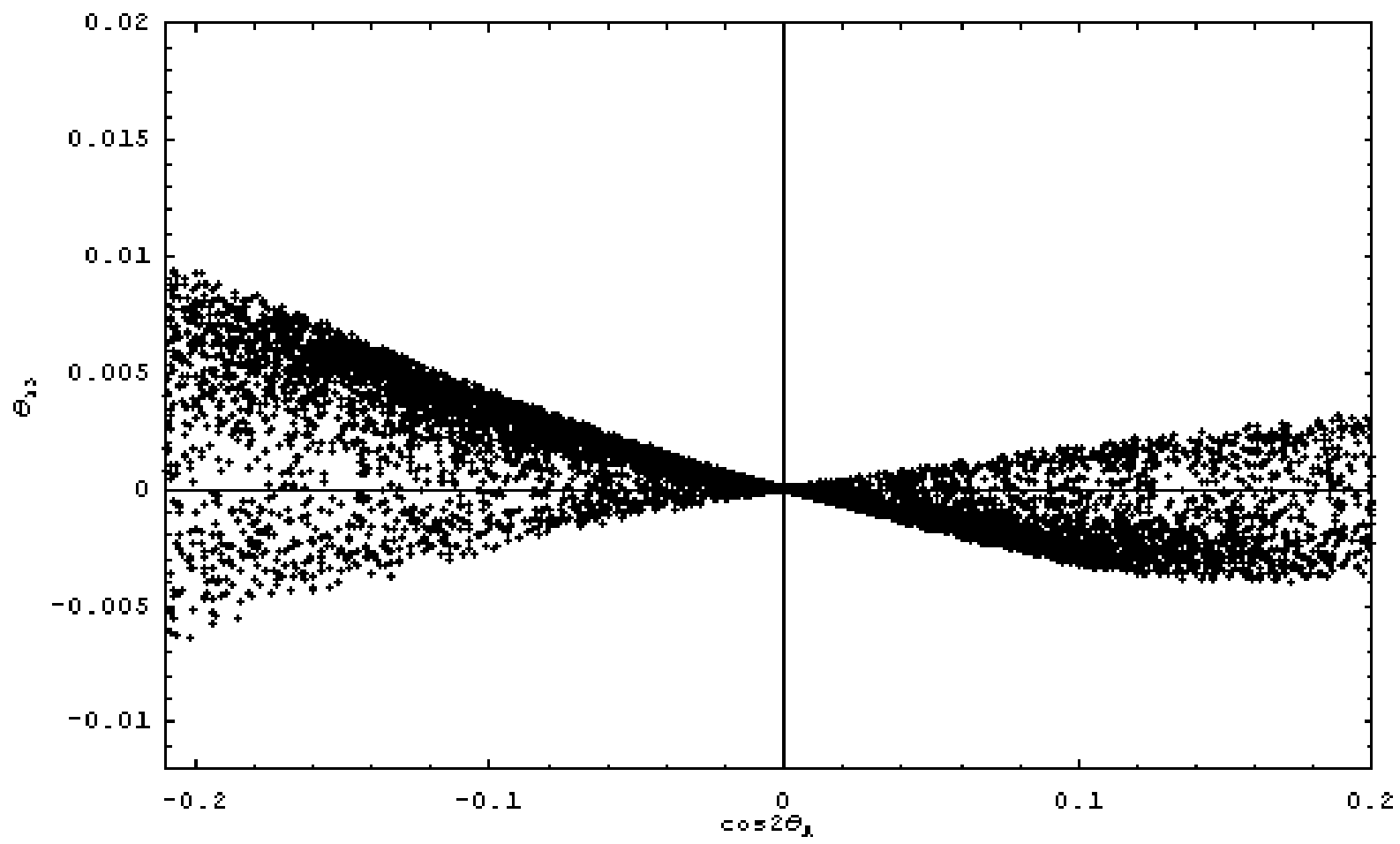

Figure 2: Departure from $\mu-\tau$ symmetry and correlation between $\theta_{13}$ and $\theta_{A}$ (Case (i)): opposite to case (ii) (figure by H. Yu)

then we have bi-maximal mixing. However, as mentioned, the observed deviation from $\pi / 4$ is rather large.

Much closer to current data is the so-called tri-bimaximal mixing scheme [112], corresponding to $\sin ^{2} \theta_{12}=1 / 3$ and leading to the following often-studied mixing matrix:

$$
U=\left(\begin{array}{ccc}
\sqrt{\frac{2}{3}} & \sqrt{\frac{1}{3}} & 0 \\
-\sqrt{\frac{1}{6}} & \sqrt{\frac{1}{3}} & \sqrt{\frac{1}{2}} \\
\sqrt{\frac{1}{6}} & -\sqrt{\frac{1}{3}} & \sqrt{\frac{1}{2}}
\end{array}\right)
$$

Models which give rise to such a matrix (for some recent attempts see [113]) are typically quite intricate and not as straightforward to construct as models leading to bi-maximal mixing.

There are other viable neutrino mass textures, including some that lead to degenerate neutrino masses. We refer readers to the literature for a more thorough discussion $([4,77]$ and references therein). The point we wish to emphasize here is that the amount of 
information we have concerning neutrino masses and leptonic mixing is still very limited. This is reflected in the fact that too many different hypothesis can be raised in order to "explain" the same set of observables. The situation is bound to change in the near future, and there is hope that the data will "select" one specific neutrino mass matrix. Our job will then be to interpret what Nature is trying to "say" through $m_{\nu}$. A more accurate determination of a few observables will already shine a significant amount of light in the currently obscure picture we are trying to obtain: (i) what is the neutrino mass hierarchy? (ii) is $\left|U_{e 3}\right|$ larger than 0.1 ? (iii) is $\left|\cos 2 \theta_{23}\right|>0.1$ ? All three of these can be answered in a next-generation $\nu_{\mu} \rightarrow \nu_{e}$ long-baseline accelerator experiment, while the second one can be addressed by a next-generation reactor neutrino experiment (with a baseline of $\mathcal{O}(1 \mathrm{~km})$ ). Of course, in order to be sure we are on the right track we also need to (iv) determine that lepton number is not a conserved symmetry. 


\section{$3 \quad \boldsymbol{\beta} \boldsymbol{\beta}_{0 \nu}$-Decay and CP Violation}

\section{$3.1 \beta \beta_{0 \nu}$ decay}

In this section, we focus our attention on what we can learn from neutrinoless double beta decay experiments. As already alluded to in the introduction, in a given theory, neutrinoless double beta decay can arise from two sources: (i) neutrino Majorana mass or/and (ii) lepton number violating interactions. While the absence of a signal in a $\beta \beta_{0 \nu}$ experiment will constrain both sources (and associated theories), a positive signal cannot necessarily be considered as evidence for one or the other exclusively. For instance, one must supplement the results from $\beta \beta_{0 \nu}$ experiment with collider experiments such as from LHC or a possible $e^{+} e^{-}$type to get a definitive information regarding the source. Alternatively, one may conduct the double beta decay experiment with different nuclei and if the matrix elements happen to differ substantially for the two sources, one may be able to disentangle the different sources. Therefore as we interpret any positive signal for $\beta \beta_{0 \nu}$ decay one must keep this in mind. Below, we discuss what we can learn about neutrino masses and mixings, once it is established that the source of the positive signal is the Majorana mass for the neutrino. The experiments with solar and atmospheric neutrinos and with reactor antineutrinos have provided data on $\theta_{12}, \theta_{23}$ and $\theta_{13}$, and on the neutrino mass squared differences driving the solar and atmospheric neutrino oscillations, $\Delta m_{12}^{2}$ and $\Delta m_{13}^{2}$. Future oscillation experiments will improve considerably the precision on these basic parameters. However, these experiments are insensitive to the nature of massive neutrinos $\nu_{j}$ which can be Dirac or Majorana particles [11,114] (see also, e.g., [23]). They cannot give information on the absolute scale of neutrino masses (i.e., on the value of $m_{1}$ ), and on the two Majorana $\mathrm{CP}$ violation phases - the latter do not enter into the expressions for the probabilities of flavor neutrino oscillations [11]. Determining the nature of massive neutrinos and obtaining information on the absolute neutrino mass scale is one of the fundamental problems in the studies of neutrino mixing.

Neutrinos are predicted to be Majorana particles in the seesaw model of neutrino mass generation. This model gives a natural explanation of the smallness of the neutrino masses and, through the leptogenesis theory, provides an explanation of the observed baryon asymmetry in the Universe, which thus is linked to the existence of neutrino mixing. The only experiments which have the potential of establishing the Majorana nature of massive neutrinos are the neutrinoless double beta decay experiments searching for the process $(A, Z) \rightarrow(A, Z+2)+e^{-}+e^{-}$(see, e.g., $\left.[23,24]\right)$. The observation of the $\beta \beta_{0 \nu}$-decay and the measurement of the corresponding $\beta \beta_{0 \nu}$-decay rate with a sufficient accuracy, would not only be a proof that the total lepton charge is not conserved in nature, but might provide also a unique information on i) the type of the neutrino mass spectrum, ii) the absolute scale of neutrino masses, and on iii) the values of the Majorana $\mathrm{CP}$ violation phases. Let us add that in supersymmetric theories with seesaw mechanism of neutrino mass generation, the rates of lepton flavor violating decays $\mu \rightarrow e+\gamma, \tau \rightarrow \mu+\gamma$ can be interestingly large (e.g., [116]) and may depend on the Majorana CP violating phases in the lepton mixing matrix (see, e.g., $[117,118]$ ). Furthermore, the values of the Majorana phases can be important for the stability under RGE running of the neutrino mass and mixing parameters, see Sec. 5.3 
Let us recall that the SK atmospheric neutrino and K2K data do not allow one to determine the sign of $\Delta m_{\mathrm{A}}^{2}$. This implies that if we identify $\Delta m_{\mathrm{A}}^{2}$ with $\Delta m_{13}^{2}$ in the case of 3-neutrino mixing, one can have $\Delta m_{13}^{2}>0$ or $\Delta m_{13}^{2}<0$. The two possibilities correspond to two different types of neutrino mass spectrum: with normal hierarchy, $m_{1}<m_{2}<m_{3}$, and with inverted hierarchy, $m_{3}<m_{1}<m_{2}$. In the case of strong inequalities between the masses, the spectra are called normal hierarchical (NH) and inverted hierarchical (IH). The NH and IH spectra correspond to $m_{1} \ll 0.02 \mathrm{eV}$ and $m_{3} \ll 0.02 \mathrm{eV}$, respectively. If $m_{1} \cong m_{2} \cong m_{3} \cong m_{0}$ and $m_{j}^{2} \gg\left|\Delta m_{\mathrm{A}}^{2}\right|, \Delta m_{\odot}^{2}$, the spectrum is quasi-degenerate (QD). The QD spectrum is realized if $m_{1,2,3}>0.20 \mathrm{eV}$ roughly requiring that the largest mass difference is about $10 \%$ of the common mass.

Under the assumptions of (1) 3-neutrino mixing, for which we have compelling evidence from the experiments with solar and atmospheric neutrinos and from the KamLAND experiment, (2) massive neutrinos $\nu_{j}$ being Majorana particles, and (3) $\beta \beta_{0 \nu}$-decay generated only by the ( $V$-A) charged current weak interaction via the exchange of the three Majorana neutrinos $\nu_{j}$, the effective Majorana mass in $\beta \beta_{0 \nu}$-decay of interest is given by (see, e.g., $[23,119])$ :

$$
\langle m\rangle_{e f f}=\left.\left|m_{1}\right| U_{e 1}\right|^{2}+m_{2}\left|U_{e 2}\right|^{2} e^{2 i \phi_{1}}+m_{3}\left|U_{e 3}\right|^{2} e^{2 i \phi_{2}} \mid
$$

where $U_{e j}, j=1,2,3$, are the elements of the first row of the lepton mixing matrix $U$, $m_{j}>0$ is the mass of the Majorana neutrino $\nu_{j}$, and $\phi_{1}$ and $\phi_{2}$ are the two Majorana CP violating phases $[12,114]$. In the case of $\mathrm{CP}$ conservation we have $e^{2 i \phi_{1,2}} \equiv \eta_{21(31)}= \pm 1$, $\eta_{i j}$ being the relative $\mathrm{CP}$ parity of the neutrinos $\nu_{i}$ and $\nu_{j}$.

One can express [120] two of the three neutrino masses, say, $m_{2,3}$, in terms of the third mass, $m_{1}$, and of $\Delta m_{\odot}^{2}$ and $\Delta m_{\mathrm{A}}^{2}$, while the elements $\left|U_{e j}\right|$ can be expressed in terms of $\theta_{\odot}$ and $\theta$ (a concise discussion of the relevant formalism can be found, e.g., in Refs. $[119,121,122])$. Within the convention employed in the present study in both cases of neutrino mass spectrum with normal and inverted hierarchy one has: $\Delta m_{\odot}^{2}=\Delta m_{12}^{2}>0$, and $m_{2}=\sqrt{m_{1}^{2}+\Delta m_{\odot}^{2}}$. In the case of normal hierarchy, $\Delta m_{\mathrm{A}}^{2}=\Delta m_{13}^{2}>0$ and $m_{3}=\sqrt{m_{1}^{2}+\Delta m_{\mathrm{A}}^{2}}$, while if the spectrum is with inverted hierarchy, $\Delta m_{\mathrm{A}}^{2}=\Delta m_{23}^{2}>0$ and thus $m_{1}=\sqrt{m_{3}^{2}+\Delta m_{\mathrm{A}}^{2}-\Delta m_{\odot}^{2}}$. For both types of hierarchy, the following relations hold: $\left|U_{\mathrm{e} 1}\right|^{2}=\cos ^{2} \theta_{\odot}\left(1-\left|U_{\mathrm{e} 3}\right|^{2}\right),\left|U_{\mathrm{e} 2}\right|^{2}=\sin ^{2} \theta_{\odot}\left(1-\left|U_{\mathrm{e} 3}\right|^{2}\right)$, and $\left|U_{\mathrm{e} 3}\right|^{2} \equiv \sin ^{2} \theta$. We denote the smallest neutrino mass as $m_{\min }$ and we have $m_{\min }=m_{1 \text { (3) }}$ for the case of normal (inverted) hierarchy. The problem of obtaining the allowed values of $\langle m\rangle_{e f f}$ given the constraints on the relevant parameters following from the neutrino oscillation data has been first studied in Ref. [120] and subsequently in a large number of papers, see, e.g., [39, 119, 123-126]. Detailed analyses were performed, e.g., in Refs. [29, 100,119, 122], and in particular in Ref. [127], where the allowed values of $\Delta m_{\mathrm{A}}^{2}, \Delta m_{\odot}^{2}, \theta_{\odot}$ and $\theta$, obtained from the most recent neutrino oscillation data, were used. The results are summarized in Tabs. 3 and 4, and in Fig. 3,

In Fig. 3 (taken from Ref. [127]) we show the allowed ranges of values of $\langle m\rangle_{e f f}$ as a function of $m_{\min }$ for the cases of $\mathrm{NH}$ and $\mathrm{IH}$ spectrum The predictions for $\langle m\rangle_{\text {eff }}$ are obtained by using the allowed at $90 \%$ C.L. (Fig. [3), values of $\Delta m_{\odot}^{2}, \theta_{\odot}$ and $\Delta m_{\mathrm{A}}^{2}$ from Refs. [128] and [130] and for three fixed values of $\sin ^{2} \theta$.

The existence of significant lower bounds on $\langle m\rangle_{e f f}$ in the cases of IH and QD spectra [131], $\langle m\rangle_{\text {eff }}^{\mathrm{HH}} \geq 10 \mathrm{meV}$ and $\langle m\rangle_{\text {eff }}^{\mathrm{HH}} \geq 43 \mathrm{meV}$, respectively [127], which lie either 


\begin{tabular}{|c|c|c|c|c|}
\hline $\sin ^{2} \theta$ & $\langle m\rangle_{\text {eff } f_{\max }}^{\mathrm{NH}}$ & $\langle m\rangle_{\text {eff } f_{\min }}^{\mathrm{IH}}$ & $\langle m\rangle_{\text {eff }}^{\mathrm{IH}}$ & $\langle m\rangle_{\text {eff }}^{\mathrm{QD}}$ \\
\hline \hline 0.0 & $2.6(2.6)$ & $19.9(17.3)$ & $50.5(44.2)$ & 79.9 \\
\hline 0.02 & $3.6(3.5)$ & $19.5(17.0)$ & $49.5(43.3)$ & 74.2 \\
\hline 0.04 & $4.6(4.3)$ & $19.1(16.6)$ & $48.5(42.4)$ & 68.5 \\
\hline
\end{tabular}

Table 3: The maximal values of $\langle m\rangle_{\text {eff }}$ (in units of meV) for the $\mathrm{NH}$ and IH spectra, and the minimal values of $\langle m\rangle_{\text {eff }}$ (in units of meV) for the IH and QD spectra, for the best fit values of the oscillation parameters and $\sin ^{2} \theta=0.0,0.02$ and 0.04 . The results for the $\mathrm{NH}$ and $\mathrm{IH}$ spectra are obtained for $\left|\Delta m_{\mathrm{A}}^{2}\right|=2.6 \times 10^{-3} \mathrm{eV}^{2}(2.0 \times$ $10^{-3} \mathrm{eV}^{2}$ - values in brackets) and $m_{1}=10^{-4} \mathrm{eV}$, while those for the QD spectrum correspond to $m_{0}=0.2 \mathrm{eV}$. (From Ref. [127]).

\begin{tabular}{|c|c|c|c|c|}
\hline $\sin ^{2} \theta$ & $\langle m\rangle_{e f f_{\max }^{\mathrm{NH}}}$ & $\langle m\rangle_{e f f_{\min }}^{\mathrm{IH}}$ & $\langle m\rangle_{\text {eff } f_{\max }}^{\mathrm{IH}}$ & $\langle m\rangle_{e f f_{\min }}^{\mathrm{QD}}$ \\
\hline \hline 0.0 & $3.7(3.7)$ & $10.1(8.7)$ & $56.3(50.6)$ & 47.9 \\
\hline 0.02 & $4.7(4.6)$ & $9.9(8.6)$ & $55.1(49.6)$ & 42.8 \\
\hline 0.04 & $5.5(5.3)$ & $11.4(9.9)$ & $54.0(48.6)$ & 45.4 \\
\hline
\end{tabular}

Table 4: The same as in Table 3 but for the $90 \%$ C.L. allowed regions of $\Delta m_{\odot}^{2}$ and $\theta_{\odot}$ obtained in [128], and of $\Delta m_{\mathrm{A}}^{2}$ obtained in Ref. [129] (Ref. [130] - results in brackets). (From Ref. [127]).

partially (IH spectrum) or completely (QD spectrum) within the range of sensitivity of the next generation of $\beta \beta_{0 \nu}$-decay experiments, is one of the most important features of the predictions of $\langle m\rangle_{e f f}$. The indicated minimal values are given, up to small corrections, by $\Delta m_{\mathrm{A}}^{2} \cos 2 \theta_{\odot}$ and $m_{0} \cos 2 \theta_{\odot}$. According to the most recent combined analyses of the solar and reactor neutrino data, including the latest SNO and KamLAND results (see, e.g., [132]) i) the possibility of $\cos 2 \theta_{\odot}=0$ is excluded at more than 6 s.d., ii) the best fit value of $\cos 2 \theta_{\odot}$ is $\cos 2 \theta_{\odot}=0.40$, and iii) at $95 \%$ C.L. one has for $\sin ^{2} \theta=0(0.02)$, $\cos 2 \theta_{\odot} \geq 0.27(0.32)$. The quoted results on $\cos 2 \theta_{\odot}$ together with the range of possible values of $\Delta m_{\mathrm{A}}^{2}$ and $m_{0}$, lead to the conclusion about the existence of significant and robust lower bounds on $\langle m\rangle_{\text {eff } f}$ in the cases of IH and QD spectrum $[127,133]$. At the same time one can always have $\langle m\rangle_{\text {eff }}=0$ in the case of neutrino mass spectrum with normal hierarchy [134]. It follows from Tabs. 3 and 4 that in this case $\langle m\rangle_{\text {eff }}$ cannot exceed $5.5 \mathrm{meV}$. This implies that the maximal value of $\langle m\rangle_{e f f}$ in the case of neutrino mass spectrum with normal hierarchy is considerably smaller than the minimal values of $\langle m\rangle_{e f f}$ for the inverted hierarchy and quasi-degenerate neutrino mass spectrum. This opens the possibility of obtaining information about the type of neutrino mass spectrum from a measurement of $\langle m\rangle_{\text {eff }} \neq 0$, or from obtaining a sufficiently stringent upper bound on $\langle m\rangle_{\text {eff }}$. In particular, a positive result in the future generation of $\beta \beta_{0 \nu}$-decay experiments with $\langle m\rangle_{\text {eff }}>10 \mathrm{meV}$ would imply that the $\mathrm{NH}$ spectrum is excluded. The uncertainty in the relevant nuclear matrix elements ${ }^{5}$ and prospective experimental errors in the values of the oscillation parameters, in $\langle m\rangle_{e f f}$, and for the case of QD spectrum in $m_{0}$, weaken, but do not invalidate, the reported results (see, e.g., Refs. [136]). If the

\footnotetext{
${ }^{5}$ Recently, encouraging results in what regards the problem of the calculation of the nuclear matrix elements have been obtained [135].
} 


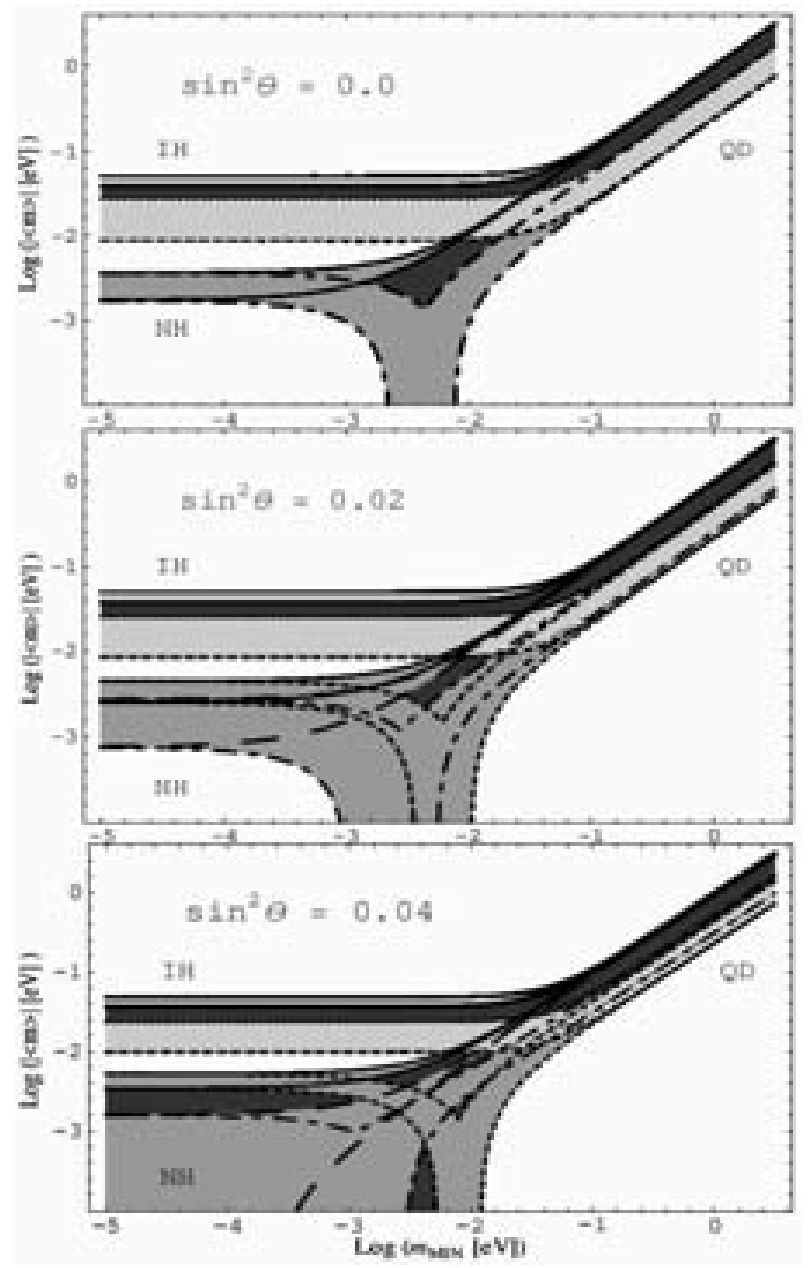

Figure 3: The dependence of $\langle m\rangle_{\text {eff }}$ on $m_{\min }$ in the case of the LMA-I solution, for normal and inverted hierarchy, and for the $90 \%$ C.L. allowed regions of $\Delta m_{\odot}^{2}$ and $\sin ^{2} \theta_{\odot}$ found in Ref. [128] and of $\Delta m_{\mathrm{A}}^{2}$ in Ref. [130], and a fixed value of $\sin ^{2} \theta=0.0(0.02)[0.04]$ in the upper (middle) [lower] panel. In the case of CP conservation, the allowed values of $\langle m\rangle_{\text {eff }}$ are constrained to lie: for i) normal hierarchy and the middle and lower panels (upper panel) - in the medium-gray and light-gray regions a) between the two lower thick solid lines (between the two lower thick solid lines) if $\eta_{21}=\eta_{31}=1, b$ ) between the two long-dashed lines (between the two lower thick solid lines) if $\eta_{21}=-\eta_{31}=1, c$ ) between the three thick dash-dotted lines and the axes (between the dash-dotted lines and the axes) if $\eta_{21}=-\eta_{31}=-1, d$ ) between the three thick short-dashed lines and the axes (between the dash-dotted lines and the axes) if $\eta_{21}=\eta_{31}=-1$; and for ii) inverted hierarchy and the middle and lower panels (upper) - in the light-gray regions a) between the two upper thick solid lines (between the two upper thick solid lines) if $\eta_{32}=\eta_{31}= \pm 1, b$ ) between the dotted and the thin dash-dotted lines (between the dotted and the thick short-dashed lines) if $\left.\eta_{32}=-\eta_{31}=1, c\right)$ between the dotted and the upper thick short-dashed lines (between the dotted and the thick short-dashed lines) if $\eta_{32}=-\eta_{31}=-1$. In the case of $\mathrm{CP}$ violation, the allowed regions for $\langle m\rangle_{\text {eff }}$ cover all the gray regions. Values of $\langle m\rangle_{e f f}$ in the dark gray regions signal CP violation.(From Ref. [127]). 
neutrino mass spectrum turned out to be of the QD type, a measurement of $\langle m\rangle_{\text {eff }}$ in $\beta \beta_{0 \nu}$-decay experiment and of $m_{0}$ in the KATRIN experiment [22] could be used, in particular, to check the validity of the light Majorana neutrino exchange mechanism for the $\beta \beta_{0 \nu}$-decay and search for indications for contributions from other types of mechanisms (see, e.g., $[25,137])$.

It follows from Fig. 3 that a measurement of $\langle m\rangle_{\text {eff }} \geq 10 \mathrm{meV}$ would either i) determine a relatively narrow interval of possible values of the lightest neutrino mass $m_{\text {min }}$, or ii) would establish an upper limit on the possible values of $m_{\text {min }}$. If a sufficiently stringent upper limit on $\langle m\rangle_{e f f}$ is experimentally obtained below $100 \mathrm{meV}$, this would lead to a significant upper limit on the possible value of $m_{\min }$.

The possibility of establishing CP violation in the lepton sector due to Majorana CP violating phases has been studied in $[126,134]$ and in much greater detail in Ref. [100]. It was found that it is very challenging ${ }^{6}$ : it requires quite accurate measurements of $\langle m\rangle_{e f f}$ and of $m_{1}$, and holds only for a limited range of values of the relevant parameters. For the IH and the QD spectra, which are of interest, the "just CP violation" region [119] — an experimental point in this region would signal unambiguously CP violation associated with Majorana neutrinos, is larger for smaller values of $\cos 2 \theta_{\odot}$. More specifically, proving that $\mathrm{CP}$ violation associated with Majorana neutrinos takes place requires, in particular, a relative experimental error on the measured value of $\langle m\rangle_{\text {eff }}$ not bigger than $(15-20) \%$, a "theoretical uncertainty" in the value of $\langle m\rangle_{\text {eff }}$ due to an imprecise knowledge of the corresponding nuclear matrix elements smaller than a factor of 2 , a value of $\tan ^{2} \theta_{\odot} \geq 0.55$, and values of the relevant Majorana $\mathrm{CP}$ violating phases $\left(2 \phi_{1,2}\right)$ typically within the ranges of $\sim(\pi / 2-3 \pi / 4)$ and $\sim(5 \pi / 4-3 \pi / 2)$ [100].

\subsection{The MNSP Lepton Mixing Matrix and CP Violation in the Lepton Sector}

It is well known that, in general, in gauge theories with massive neutrinos the MNSP lepton mixing matrix results from a product of two matrices:

$$
U=U_{\ell}^{\dagger} U_{\nu}
$$

where $U_{\ell}$ and $U_{\nu}$ are two $3 \times 3$ unitary matrices: $U_{\ell}$ arises from the diagonalization of the charged lepton mass matrix, while $U_{\nu}$ diagonalizes the neutrino Majorana mass term. Any $3 \times 3$ unitary matrix contains 3 moduli and 6 phases and can be written as [138] (see also [139]):

$$
U=e^{i \Phi} P \tilde{U} Q
$$

where $P \equiv \operatorname{diag}\left(1, e^{i \phi}, e^{i \omega}\right)$ and $Q \equiv \operatorname{diag}\left(1, e^{i \rho}, e^{i \sigma}\right)$ are diagonal phase matrices having 2 phases each, and $\tilde{U}$ is a unitary "CKM-like" matrix containing 1 phase and 3 angles. The charged lepton Dirac mass term, $M_{\ell}$, is diagonalized by a bi-unitary transformation:

$$
M_{\ell}=U_{L} M_{\ell}^{\operatorname{diag}} U_{R}^{\dagger}
$$

\footnotetext{
${ }^{6} \mathrm{~A}$ very pessimistic conclusion about the prospects to establish $\mathrm{CP}$ violation in the lepton sector due to Majorana CP violating phases from a measurement of $\langle m\rangle_{e f f}$ and, e.g., of $m_{0}$, was reached in [99].
} 
where $U_{L, R}$ are $3 \times 3$ unitary matrices and $M_{\ell}^{\text {diag }}$ is the diagonal matrix containing the masses of the charged leptons. Casting $U_{L, R}$ in the form (17), i.e., $U_{L, R}=e^{i \Phi_{L, R}} P_{L, R} \tilde{U}_{L, R} Q_{L, R}$, we find

$$
M_{\ell}=e^{i\left(\Phi_{L}-\Phi_{R}\right)} Q_{L} \tilde{U}_{L} P_{L} M_{\ell}^{\operatorname{diag}} Q_{R}^{\dagger} \tilde{U}_{R}^{\dagger} P_{R}^{\dagger} .
$$

The term $P_{L} M_{\ell}^{\text {diag }} Q_{R}^{\dagger}$ contains only 2 relative phases, which can be associated with the right-handed charged lepton fields. The three independent phases in $e^{i\left(\Phi_{L}-\Phi_{R}\right)} Q_{L}$ can be absorbed by a redefinition of the left-handed charged lepton fields. Therefore, $U_{\ell}$ is effectively given by $\tilde{U}_{L}$ and contains three angles and one phase.

The neutrino mass matrix $M_{\nu}$ is diagonalized via

$$
M_{\nu}=U_{\nu}^{*} M_{\nu}^{\text {diag }} U_{\nu}^{\dagger}
$$

The unitary matrix $U_{\nu}$ can be written in the form (17). It is not possible to absorb phases in the neutrino fields since the neutrino mass term is of Majorana type [12,114]. Thus,

$$
U=U_{\ell}^{\dagger} U_{\nu}=e^{i \Phi \nu} \tilde{U}_{\ell}^{\dagger} P_{\nu} \tilde{U}_{\nu} Q_{\nu}
$$

The common phase $\Phi_{\nu}$ has no physical meaning and we will ignore it. Consequently, in the most general case, the elements of $U$ given by Eq. (21) are expressed in terms of six real parameters and six phases in $\tilde{U}_{\ell}$ and $U_{\nu}$. Only six combinations of those - the three angles and the three phases of $U$, are observable, in principle, at low energies. Note that the two phases in $Q_{\nu}$ are "Majorana-like", since they will not appear in the probabilities describing the flavor neutrino oscillations $[12,114]$. Note also that if $U_{\ell}=\mathbb{1}$, the phases in the matrix $P_{\nu}$ can be eliminated by a redefinition of the charged lepton fields.

If one assumes that, e.g., $\tilde{U}_{\nu}$ is bimaximal,

$$
\tilde{U}_{\nu} \equiv U_{\text {bimax }}=\left(\begin{array}{ccc}
\frac{1}{\sqrt{2}} & \frac{1}{\sqrt{2}} & 0 \\
-\frac{1}{2} & \frac{1}{2} & \frac{1}{\sqrt{2}} \\
\frac{1}{2} & -\frac{1}{2} & \frac{1}{\sqrt{2}}
\end{array}\right),
$$

which permits a rather simple explanation of the smallness of $\sin \theta_{13}$ and the deviation of $\theta_{\odot}$ from $\pi / 4$, then $\tilde{U}_{\nu}$ is real. In this case the three angles and the Dirac phase in the neutrino mixing matrix $U$ will depend in a complicated manner on the three angles and the phase in $\tilde{U}_{\ell}$ and on the two phases in $P_{\nu}$. The two Majorana phases will depend in addition on the parameters in $Q_{\nu}$. See [102] for details.

It should be emphasized that the form of $U$ given in Eq. (21) is the most general one. A specific model in the framework of which Eq. (21) is obtained might imply symmetries or textures both in $m_{\ell}$ and $M_{\nu}$, which will reduce the number of independent parameters in $U_{\ell}^{\dagger}$ and/or $U_{\nu}$.

In the scheme with three massive Majorana neutrinos under discussion there exist three rephasing invariants related to the three $\mathrm{CP}$ violating phases in $U, \delta$ and $\phi_{1,2}$ [140-144]. The first is the standard Dirac one $J_{C P}[140]$, associated with the Dirac phase $\delta$ :

$$
J_{C P}=\operatorname{Im}\left\{U_{e 1} U_{\mu 2} U_{e 2}^{*} U_{\mu 1}^{*}\right\} .
$$

It determines the magnitude of CP violation effects in neutrino oscillations [141]. Let us note that if $U_{\ell}=\mathbb{1}$ and $\tilde{U}_{\nu}$ is a real matrix, one has $J_{C P}=0$. 
The two additional invariants, $S_{1}$ and $S_{2}$, whose existence is related to the Majorana nature of massive neutrinos, i.e., to the phases $\alpha$ and $\beta$, can be chosen as $[142,144]$ (see also $[119])^{7}$ :

$$
S_{1}=\operatorname{Im}\left\{U_{e 1} U_{e 3}^{*}\right\} \quad, \quad S_{2}=\operatorname{Im}\left\{U_{e 2} U_{e 3}^{*}\right\} .
$$

If $S_{1} \neq 0$ and/or $S_{2} \neq 0, \mathrm{CP}$ is not conserved due to the Majorana phases $\phi_{1}$ and/or $\phi_{2}$. The effective Majorana mass in $\beta \beta_{0 \nu}$-decay, $\langle m\rangle_{e f f}$, depends, in general, on $S_{1}$ and $S_{2}$ [119] and not on $J_{C P}$. Let us note, however, even if $S_{1,2}=0$ (which can take place if, e.g., $\left.\left|U_{e 3}\right|=0\right)$, the two Majorana phases $\phi_{1,2}$ can still be a source of CP non-conservation in the lepton sector provided $\operatorname{Im}\left\{U_{e 1} U_{e 2}^{*}\right\} \neq 0$ and $\operatorname{Im}\left\{U_{\mu 2} U_{\mu 3}^{*}\right\} \neq 0$ [144].

Let us denote the phase in $\tilde{U}_{\ell}$ by $\psi$. We will include it in $\tilde{U}_{\ell}$ in the same way this is done for the phase $\delta$ in the standard parametrization of the $U$ matrix. If we write $P_{\nu}=$ $\operatorname{diag}\left(1, e^{i \phi}, e^{i \omega}\right)$ and $Q_{\nu} \equiv \operatorname{diag}\left(1, e^{i \rho}, e^{i \sigma}\right)$, the Dirac phase $\delta$ in $U$, which has observable consequences in neutrino oscillation experiments, is determined only by the "Dirac phase" in $\tilde{U}_{\nu}$ and the phases $\psi, \phi$ and $\omega$. The Majorana phases in $U$ receive contributions also from the two remaining phases $\rho$ and $\sigma$. Allowing the phases $\delta$ and $\phi_{1,2}$ to vary between 0 and $2 \pi$, permits to constrain (without loss of generality) the mixing angles in $\tilde{U}_{\ell}, \theta_{i j}$, to lie between 0 and $\pi / 2$.

There are interesting specific cases in which there are direct relations between all 3 $\mathrm{CP}$ violating phases in the $U$ matrix [102].

\footnotetext{
${ }^{7}$ We assume that the fields of massive Majorana neutrinos satisfy Majorana conditions which do not contain phase factors.
} 


\section{Testing Seesaw Models}

Although it is far from clear that the seesaw mechanism [67-70] is responsible for neutrino masses, most physicists consider that it is by far the most elegant mechanism. It fits very well into the big picture of other areas of particle physics such as supersymmetry, grand unification etc. It is therefore important to discuss how we can test the seesaw models. Evidently, such tests are indirect, since the right-handed electroweak-singlet neutrinos are much too heavy to be produced at colliders. In this section, we consider two aspects of the seesaw mechanism: (i) indirect signals of seesaw mechanism in lepton flavor violating processes, which can be measured in near future; (ii) leptogenesis, which can give us a hint as to the $\mathrm{CP}$ violating phases in the lepton sector as well as perhaps the spectrum of the $\mathrm{RH}$ neutrinos. The presence of $\mathrm{CP}$ violating phases needed for leptogenesis (see Sec. 4.2) in turn can lead to CP violating low energy observables in the seesaw models. We explore these probes of the seesaw mechanism in this section.

\subsection{Lepton Flavor Violation and Lepton Electric Dipole Mo- ments}

Neutrino oscillation experiments have revealed that the violation of flavor symmetry is much greater in the lepton sector than in the quark sector. We will discuss how this flavor violation manifests itself via the seesaw mechanism in other observable quantities where lepton flavor and/or CP are violated. As we are going to discuss, among the laboratory observables particularly interesting are Lepton Flavor Violating (LFV) decays, like $\mu \rightarrow e \gamma$ and $\tau \rightarrow \mu \gamma$, and Lepton Electric Dipole Moments (LEDM), like $d_{e}$ and $d_{\mu}$.

\begin{tabular}{|c||c|c|c|c|}
\hline & $\mathrm{BR}(\mu \rightarrow e \gamma)$ & $\mathrm{BR}(\tau \rightarrow \mu \gamma)$ & $d_{e}[\mathrm{e} \mathrm{cm}]$ & $d_{\mu}[\mathrm{e} \mathrm{cm}]$ \\
\hline \hline present [145] & $<1.210^{-11}$ & $<1.110^{-6}$ & $<1.510^{-27}$ & $<10^{-18}$ \\
\hline planned [146] & $<10^{-14}$ & $<10^{-8}$ & $<10^{-29(-32)}$ & $<10^{-24(-26)}$ \\
\hline
\end{tabular}

Table 5: Present status and future prospects for LFV decays and LEDM.

Searches for LFV decays and for LEDM are experimentally very promising, since the present upper bounds could be strengthened by many orders of magnitude, as summarized in Table 5. Their impact on theory is also very promising: finding LFV and LEDM means discovering new low energy physics beyond the SM supplemented with the seesaw $[61,147]$, like e.g. supersymmetry. This can be easily understood by identifying the SM with the operators of dimension $d \leq 4$ of a low energy effective theory valid up to a cutoff $\Lambda$. Flavor and CP are accidentally conserved in the leptonic sector of the SM, hence there is no room for neutrino oscillations nor for LFV decays and LEDM. Their possible sources have to be found among the operators of $d>4$ : neutrino masses arise from the $d=5$ operator $\nu^{T} C^{-1} \nu\left\langle H^{0}\right\rangle^{2} / \Lambda$, while LFV decays and LEDM from the $d=6$ operator $\bar{\ell} \sigma^{\mu \nu}\left(1+\gamma_{5}\right) \ell F_{\mu \nu}\left\langle H^{0}\right\rangle / \Lambda^{2}$. In the seesaw lepton flavour is no longer conserved but $\Lambda \sim M_{R} \lesssim 10^{15} \mathrm{GeV}$ and, as a consequence, the $d=6$ operator above is so strongly suppressed that its effects are negligibly small [61,147]. However, if additional physics is present at smaller mass scales and if this additional physics violates LF and/or CP, the 
suppression is milder and LFV decays and LEDM could be raised up to the experimentally interesting range.

This enhancement due to new low-energy physics is precisely what happens in lowenergy supersymmetry ${ }^{8}$ where, due to loops with sleptons and gauginos, the $d=6$ operator is suppressed by powers of $m_{\mathrm{SUSY}}$. The experimental limits on LFV decays and LEDM then imply such severe constraints [150] on the amount of LF and CP violations in slepton masses (defined in the basis where charged fermions are diagonal), that one would expect LF and CP to be exact symmetries of the supersymmetry breaking mass terms defined at the appropriate cutoff scale (the Planck scale for supergravity, the messenger mass for gauge mediation, etc). It is important to stress that, if below this scale there are $\mathrm{LF}$ and $\mathrm{CP}$ violating Yukawa interactions, in the running down to $m_{\mathrm{SUSY}}$ they nevertheless induce a small amount of LF and CP violations in slepton masses.

It is well known that this is the case for the seesaw interactions of the right-handed neutrinos [151] and/or the GUT interactions of the heavy triplets [152]. Remarkably enough, this radiative contribution to the LFV decays and LEDM, which essentially depends on the supersymmetric spectrum and on the pattern of the Yukawa interactions, might be close to or even exceed the present or planned experimental limits. Clearly, this has an impact on seesaw models, possibly embedded also in a GUT framework. In the following we will discuss separately the case of type I and type II seesaw.

\subsubsection{Type I Seesaw: LFV}

For type I seesaw, in the low energy basis where charged leptons are diagonal, the $i j$ mass term of $L$-sleptons, $m^{2}{ }_{i j}^{L L}$, is the relevant one in the decay $\ell_{i} \rightarrow \ell_{j} \gamma$. Assuming for the sake of simplicity the mSUGRA [153] spectrum at $M_{\mathrm{Pl}}$, one obtains at the leading $\log$ [151] (see also [154]):

$$
m^{2}{ }_{i j}^{L L}=\frac{1}{8 \pi^{2}}\left(3 m_{0}^{2}+2 A_{0}^{2}\right) C_{i j}, \quad C_{i j} \equiv \sum_{k}\left(Y_{\nu}\right)_{i k}\left(Y_{\nu}\right)_{j k}^{*} \ln \frac{M_{\mathrm{Pl}}}{M_{k}},
$$

where $Y_{\nu}=M_{\nu}^{D} / v_{\mathrm{wk}}$ and $m_{0}$ and $A_{0}$ are the universal scalar masses and trilinear couplings at $M_{\mathrm{Pl}}$, respectively, and we have chosen the basis where $M_{R}$ is diagonal. For the full RG results, see [155]. The seesaw model dependence thus resides in $\left|C_{i j}\right|$, and an experimental limit on $\operatorname{BR}\left(\ell_{i} \rightarrow \ell_{j} \gamma\right)$ corresponds to an upper bound on $\left|C_{i j}\right|$. For $\mu \rightarrow e \gamma$ and $\tau \rightarrow \mu \gamma$ this bound [156] is shown in Fig. 4 as a function of the lightest charged slepton and gaugino masses.

It has been shown that many seesaw models predict $\left|C_{\mu e}\right|$ and/or $\left|C_{\tau \mu}\right|$ close to the experimentally accessible range $[157,158]$ and, in particular, this might be the case for models based on $U(1)$ flavor symmetries [159]. To reduce the uncertainty due to the supersymmetric spectrum, it is interesting to exploit the correlation between LFV decays and muon $g-2[160]$ or neutralino dark matter [161].

Planned searches could also help in discriminating between categories of seesaw models [156]. To give some hints on the latter issue consider, e.g., hierarchical eigenvalues of $Y_{\nu}$. The different $N^{c}$ thresholds can then be neglected and in first approximation one has

\footnotetext{
${ }^{8}$ It can also happen in quite different theories, such as extended technicolor $[148,149]$.
} 
Upper limit on $\left|C_{\tau \mu}\right|$

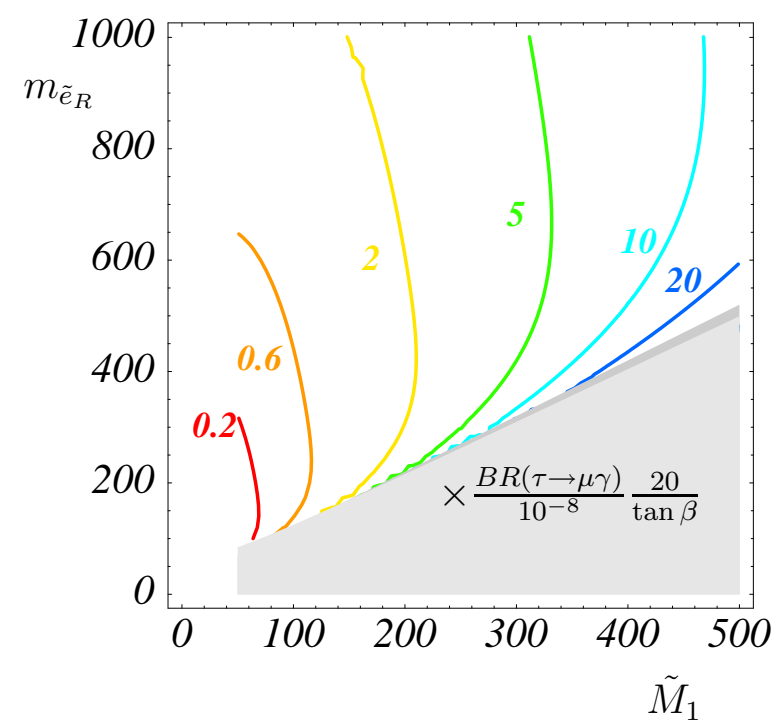

Upper limit on $\left|C_{\mu e}\right|$

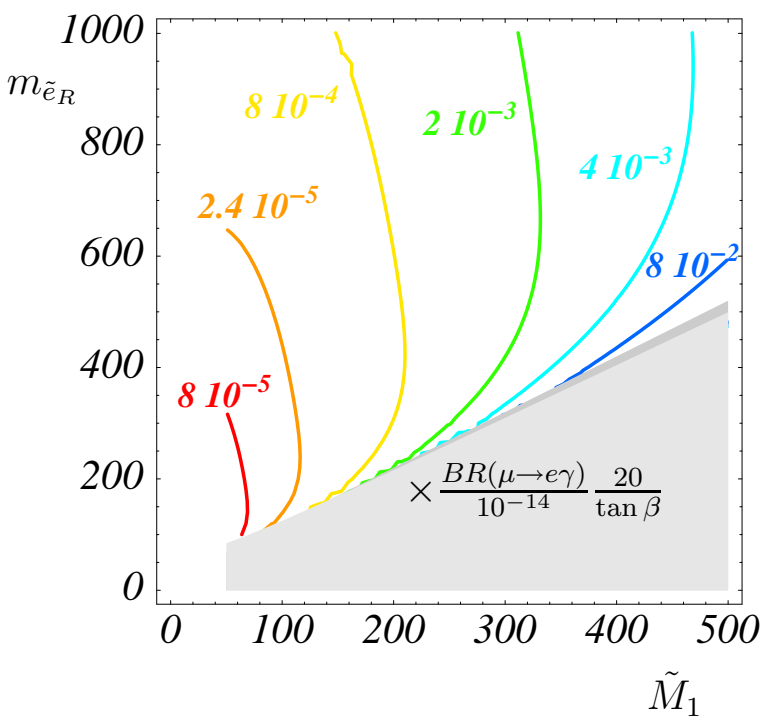

Figure 4: Upper bounds on $\left|C_{\tau \mu}\right|,\left|C_{\mu e}\right|$ in the plane $\left(\tilde{M}_{1}, m_{\tilde{e}_{R}}\right)$, respectively the bino and right slepton masses expressed in $\mathrm{GeV}$. We choose as reference values the planned experimental sensitivities $B R(\tau \rightarrow \mu \gamma)<10^{-8}, B R(\mu \rightarrow e \gamma)<10^{-14}$, with $\tan \beta=20$, reminding how to adapt the numbers in the plots for different values of these parameters. We also assume mSUGRA with $m_{0}=A_{0}$ and $\mu$ from radiative electroweak symmetry breaking. The plots are adapted from those in the last Ref. of [156].

$\left|C_{i j}\right| \approx\left|V_{L i 3}\right|\left|V_{L j 3}\right| y_{3}^{2} \log \left(M_{\mathrm{Pl}} / M_{3}\right)$, where $V_{L}$ is the lepton analog of the CKM mixing matrix. In $S O(10)$-inspired models $y_{3}=y_{t} \sim 1$, and the model dependence essentially resides in the magnitude of $\left|V_{L i 3}\right|\left|V_{L j 3}\right|$, namely on the amount of $L F$ violation present in the left-mixings of $Y_{\nu}$. Under the above assumptions $\left|C_{\tau \mu}\right|=\mathcal{O}\left(10 \times\left|V_{L 32}\right|\right)$. If at high energy LF is strongly violated in the $\tau-\mu$ sector (models with 'lopsided' $y_{\nu}$ as the one studied in [162]) planned searches for $\tau \rightarrow \mu \gamma$ could be successful for a significant region of the supersymmetric parameter space. If this violation is on the contrary tiny like in the quark sector - in which case the large LF violation observed at low energy purely arises from a magnification effect of the seesaw [163] $-\tau \rightarrow \mu \gamma$ would not be observed. Progress in the experimental sensitivity to the latter decay would thus offer precious information. The prediction for $\mu \rightarrow e \gamma$, linked to the product $\left|V_{L 23}\right|\left|V_{L 13}\right|$, is more model dependent but, on the other hand, the present experimental bound is already very severe. For instance, simple $U(1)$ flavor symmetries, those with all lepton charges of the same sign, predict $\left|C_{\mu e}\right|=\mathcal{O}\left(10 \times \Delta m_{\odot}^{2} / \Delta m_{\mathrm{A}}^{2}\right)$ and for LMA are already in crisis $[156,159]$. Since the present limit corresponds to a significantly smaller degree of LFV at high energy, this means that a much richer flavor symmetry has to be at work. Notice also that in the future we could test $\left|C_{\mu e}\right|$ up to the CKM-level [164]; in fact, if $y_{3}=\mathcal{O}(1)$ and $V_{L} \approx V_{C K M}$, then $C_{\mu e}=\mathcal{O}\left(10^{-3}\right)$ which is well inside Fig. 4. 


\subsubsection{Type I Seesaw: EDM}

Let us now discuss the consequences of type I seesaw models for lepton EDM. It is well known that in the simplest supersymmetric models (with or without neutrino mass) the dipole moments of electrons and muons obey a simple scaling law $d_{e} / d_{\mu} \approx m_{e} / m_{\mu}$. Given the present bound on $d_{e}$, this implies $d_{\mu}<10^{-25} \mathrm{e} \mathrm{cm}$, which is at the level of the best experimental prospects.

Things can change in seesaw models due to the fact that interactions involving righthanded neutrinos via radiative corrections can affect the scaling law. In type I seesaw with degenerate $N^{c}$, the radiative contributions to $d_{e}$ and $d_{\mu}$ still preserve the scaling law. However, with hierarchical $N^{c}$ this proportionality is broken due to threshold effects arising from both the flavor conserving $A$-term contribution [165] and by the FV doubleinsertion contributions $[166,167]$, which dominate for $\tan \beta>10$. Nevertheless, if only the type I seesaw radiative contributions are taken into account, $d_{e}$ and $d_{\mu}$ turn out to be barely at hand of future experimental searches (but can be for very particular textures [165]).

Discovering LEDM would then suggest the presence of additional particles and interactions beyond those of the supersymmetric type I seesaw. The heavy color triplets of GUT theories are excellent candidates for this $[152,166,168]$. In particular, it turns out that the limits on $d_{e}$ are competitive with those on proton lifetime in constraining the pattern of GUT theories where heavy triplets and right-handed neutrinos are simultaneously present [169].

\subsubsection{Type II Seesaw}

We will now consider a class of models where the right-handed neutrino mass arises from a renormalizable coupling of the form $f N N \Delta_{R}$, where $N$ is a right-handed neutrino, $f$ is a coupling constant and $\Delta_{R}$ is a Higgs field whose vacuum expectation value (vev) gives mass to the right-handed neutrino. This is a natural feature of models with asymptotic parity conservation, such as those based on $S U(2)_{L} \times S U(2)_{R} \times U(1)_{B-L}$ or any higher gauge group such as $S O(10)$, where the $\Delta_{R}$ field is part of an $S U(2)_{R}$ triplet field. Parity invariance then implies that we also have an $f \nu \nu \Delta_{L}$ coupling term as a parity partner of the $N N \Delta_{R}$ coupling. In this class of theories, whenever $\Delta_{R}$ acquires a vev, so does $\Delta_{L}$

and they are related by the formula $\left\langle\Delta_{L}\right\rangle \equiv v_{L}=\frac{v_{\mathrm{wk}}^{2}}{\gamma v_{R}}$, where $v_{\mathrm{wk}}$ is the weak scale, $v_{R}$ is the $\Delta_{R}$ vev and $\gamma$ is a coupling constant in the Higgs potential. The $\Delta_{L}$ vev contributes a separate seesaw suppressed Majorana mass to neutrinos leading to the type II seesaw formula (see Eq. (44) ) [73]. In the case where right-handed Majorana masses are heavy enough, the second term in the type II seesaw formula can be negligible, and the first term, $M_{L}=f v_{L}$, is dominant. We will call this type II seesaw. The type II seesaw gives rise to the most simple explanation of the neutrino sector and is phenomenologically very successful especially when we try to construct realistic models.

The simplest model that can be imagined for type II seesaw has just MSSM and righthanded neutrinos below the GUT scale and hence there is no new symmetry breaking scale. The right-handed neutrino masses can have hierarchies and therefore get decoupled at different scales below the GUT scale. Due to the radiative corrections from the RGEs, the flavor-violating pieces present in $Y_{\nu}$ and $f$ get transmitted to the flavor universal scalar 
masses and produce lepton flavor violation. The $f$ term contribution is the additional contribution that is typical of type II seesaw models [170]:

$$
\begin{aligned}
d Y_{e} / d t & =\frac{1}{16 \pi^{2}}\left(Y_{\nu} Y_{\nu}^{\dagger}+\cdots\right) Y_{e} \\
d Y_{\nu} / d t & =\frac{1}{16 \pi^{2}}\left(f f^{\dagger}+\cdots\right) Y_{\nu} \\
d m_{L L}^{2} / d t & =\frac{1}{16 \pi^{2}}\left(Y_{\nu} Y_{\nu}^{\dagger} m_{L L}^{2}+m_{L L}^{2} Y_{\nu} Y_{\nu}^{\dagger}+\cdots\right) .
\end{aligned}
$$

Here, $m_{L L}^{2}$ represents the soft left-handed scalar masses. The flavor non-diagonal pieces generate the lepton flavor violation. In the type II seesaw the structure $f$ coupling generates the neutrino mixing parameters. Both $f$ and $Y_{\nu}$ are determined by the particular model which explains the quark and lepton masses.

In order to calculate the BRs of $\mu \rightarrow e \gamma, \tau \rightarrow \mu \gamma$ and the electric dipole moments for the electron and muon, we use the minimal SUGRA universal boundary conditions at the GUT scale. The unifying framework of $S O(10)$ has been chosen and the values of quark masses and the CKM CP violation are satisfied. The values of the universal scalar mass $m_{0}$, universal gaugino mass $m_{1 / 2}$, universal trilinear term $A_{0}, \tan \beta$ and the sign of $\mu$ as free parameters determine the final result. The assumption of universality allows us to probe the flavor violation originating from the neutrino sector. We also assume that there is no phase associated with the SUSY breaking. The Yukawa and/or the Majorana couplings are responsible for $\mathrm{CP}$ violation in these models.

The mSUGRA parameter space is constrained by the experimental lower limit on $m_{h}$, the measurements of $b \rightarrow s \gamma$ and the recent results on dark matter relic density [33]. For low $\tan \beta$, the parameter space has a lower bound on $m_{1 / 2}$ stemming from the light Higgs mass bound of $m_{h} \geq 114 \mathrm{GeV}$. For larger $\tan \beta$ the lower bound on $m_{1 / 2}$ is obtained by the CLEO constraint on $\mathrm{BR}(b \rightarrow s \gamma)$. The lightest neutralino is the dark matter candidate in this model and we satisfy the $2 \sigma$ range of the recent relic density constraint $\Omega_{\mathrm{CDM}}=$ $0.1126_{-0.009}^{+0.008}[33]$ in the parameter space. The allowed parameter space of mSUGRA mostly reduces to the neutralino-stau co-annihilation region for $m_{0}, m_{1 / 2} \leq 1000 \mathrm{GeV}$ and when we satisfy the relic density constraint, $m_{0}$ gets determined within a very narrow band. For example, $m_{0}$ varies between $60-100 \mathrm{GeV}$ for $A_{0}=0$ line in the graph. In Figs. 5 [8], we show $\operatorname{BR}[\mu \rightarrow e \gamma]$ and $\operatorname{BR}[\tau \rightarrow \mu \gamma]$ as a function of $m_{1 / 2}$ for different values of $A_{0}$. We find that the $\mathrm{BR}$ is large in most of the parameter space and can be observable. In addition, $\operatorname{BR}[\tau \rightarrow \mu \gamma]$ can also be observable in the near future. The figures demonstrate that lepton flavor violation typically increases with increasing $\tan \beta$.

The electron EDM is plotted in Fig. 17. We find that the maximum value of EDM is $\sim 10^{-31} \mathrm{e} \mathrm{cm}$. The muon EDM is shown in Fig. 8 and the maximum value shown is about $10^{-29} \mathrm{e} \mathrm{cm}$. The scaling is broken in this model. We do not assume any new CP phases in SUSY parameters, hence all CP phases arise from the Yukawa and Majorana couplings.

It is clear that if the seesaw mechanism eventually turns out to be the explanation of small neutrino mass, the crucial question becomes whether it is of type I or type II. One may then use lepton flavor violation as a way to discriminate between these two possibilities. 


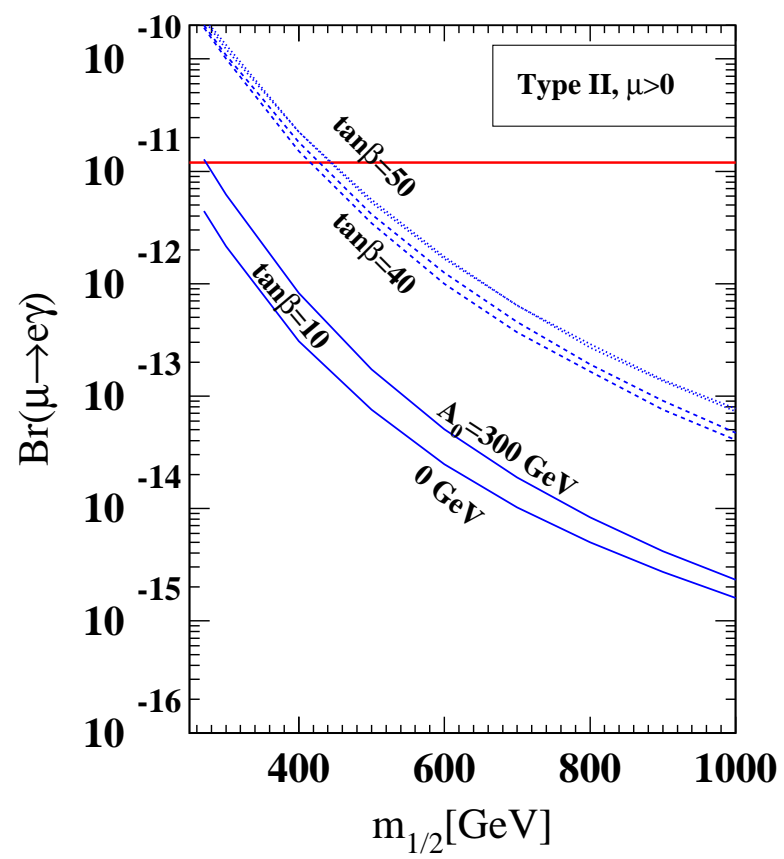

Figure 5: $\operatorname{BR}[\mu \rightarrow e \gamma]$ is plotted as a function of $m_{1 / 2}$ for different values $A_{0}$ and $\tan \beta=10,40$ and 50 in pure type II seesaw.

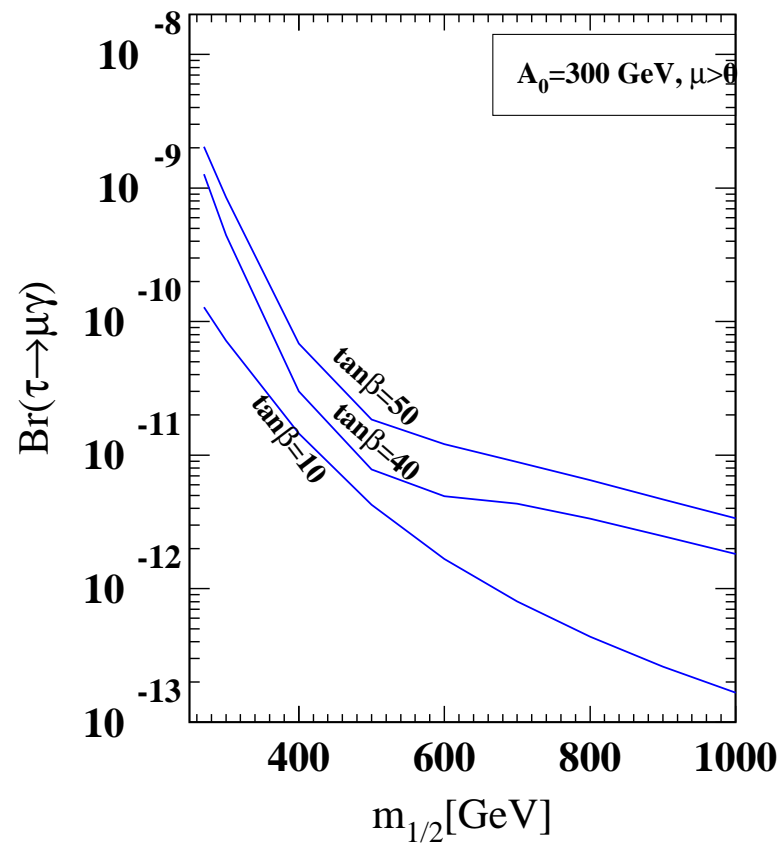

Figure 6: $\operatorname{BR}[\tau \rightarrow \mu \gamma]$ is plotted as a function of $m_{1 / 2}$ for $\tan \beta=10,40$ and 50 in pure type II seesaw. 


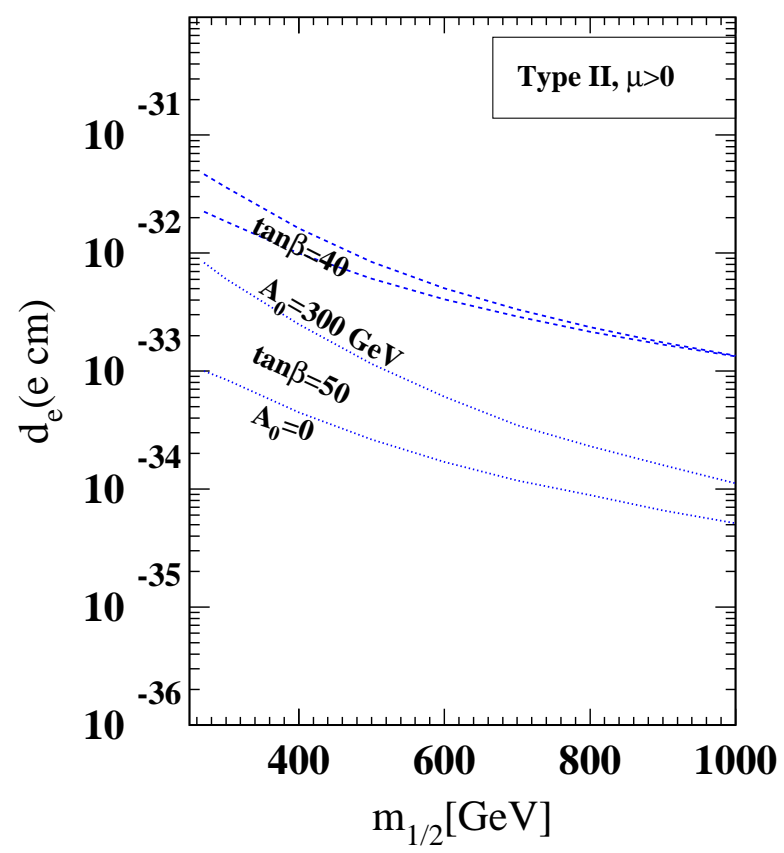

Figure 7: The electron EDM is plotted as a function of $m_{1 / 2}$ for different values $A_{0}$ and $\tan \beta=40$ and 50 in pure type II seesaw.

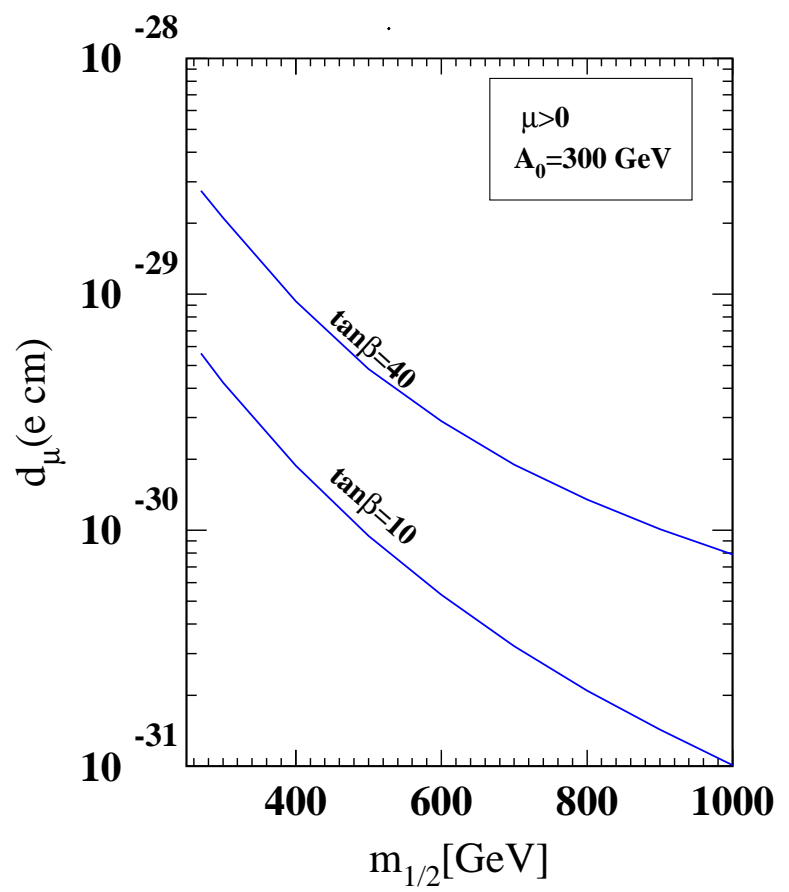

Figure 8: The muon EDM is plotted as a function of $m_{1 / 2}$ for $\tan \beta=10,40$ and 50 in pure type II seesaw. 


\subsection{Leptogenesis in the Type I Seesaw}

The origin of matter is a fundamental puzzle of cosmology and particle physics. The seesaw provides many mechanisms to generate this excess; we discuss what we can learn about neutrino physics, as well as the pattern of right-handed neutrino masses, from the observed baryon asymmetry.

Three ingredients are required to generate the observed Baryon Asymmetry of the Universe [171]: baryon number violation, $\mathrm{C}$ and $\mathrm{CP}$ violation and some out-of-thermal equilibrium dynamics. The seesaw model [66-70], which was introduced to give small neutrino masses, naturally satisfies these requirements, producing the baryon asymmetry by the "leptogenesis" mechanism [172]. It is interesting to investigate the relation between the requirements of successful leptogenesis and the observable neutrino mass and mixing matrices. In particular, does the $\mathrm{CP}$ violation that could be observed in neutrino oscillations bear any relation to leptogenesis? Do the Majorana phases that appear in neutrinoless double beta decay experiments do so?

The next subsection reviews the thermal leptogenesis scenario, focusing on the Type I seesaw, with hierarchical RH neutrinos $\left(M_{1} \lesssim M_{2,3} / 10\right)$. The relation with light neutrino parameters in type I seesaw models with three generations is discussed in Subsection 4.2.2. The situation in type II seesaw is given in Section 4.3, and the case of quasi-degenerate $N_{R}$ masses is discussed in subsection 4.5 .

\subsubsection{Thermal Leptogenesis}

The idea of leptogenesis is to use the lepton number violation of the $N_{R}$ Majorana masses, in conjunction with the $B+L$ violation contained in the Standard Model [173], to generate the baryon asymmetry. The most cosmology-independent implementation is "thermal leptogenesis" [172, 174, 175, 182,183] which will be reviewed in the following paragraph. Other leptogenesis scenarios, where the initial number density of (s)neutrinos is produced non-thermally (by inflaton decay, scalar field dynamics,...) depend on additional parameters of the cosmological model.

If the temperature $T_{R H}$ of the thermal bath after inflation is $\gtrsim M_{R 1}$, the lightest $N_{R}$ will be produced by scattering. If the $N_{R}$ subsequently decay out of equilibrium, a CP asymmetry $\epsilon_{1}$ in the decay produces a net asymmetry of Standard Model leptons. This asymmetry is partially transformed into a baryon asymmetry by the non-perturbative $B+L$ violation [184]. Thermal leptogenesis has been studied in detail $[174,175,177,182$, $183,185]$; the baryon to entropy ratio produced is

$$
Y_{B} \simeq C \kappa \frac{n}{s} \epsilon_{1}
$$

where $\kappa \leq 1$ is an efficiency factor to be discussed in a moment, $n / s \sim 10^{-3}$ is the ratio of the $N_{R}$ equilibrium number density to the entropy density, and $\epsilon_{1}$ is the CP asymmetry in the $N_{R 1}$ decay. $C \sim 1 / 3$ tells what fraction of the produced lepton asymmetry is reprocessed into baryons by the $B+L$ violating processes. $Y_{B}$ depends largely on three parameters: the $N_{R 1}$ mass $M_{R 1}$, its decay rate $\Gamma_{1}$, and the $\mathrm{CP}$ asymmetry $\epsilon_{1}$ in the decay. The decay rate $\Gamma_{j}$ of $N_{R j}$ can be conveniently parametrized as $\Gamma_{j}=\frac{\left[Y_{\nu}^{\dagger} Y_{\nu}\right]_{j j} M_{j}}{8 \pi} \equiv$ 

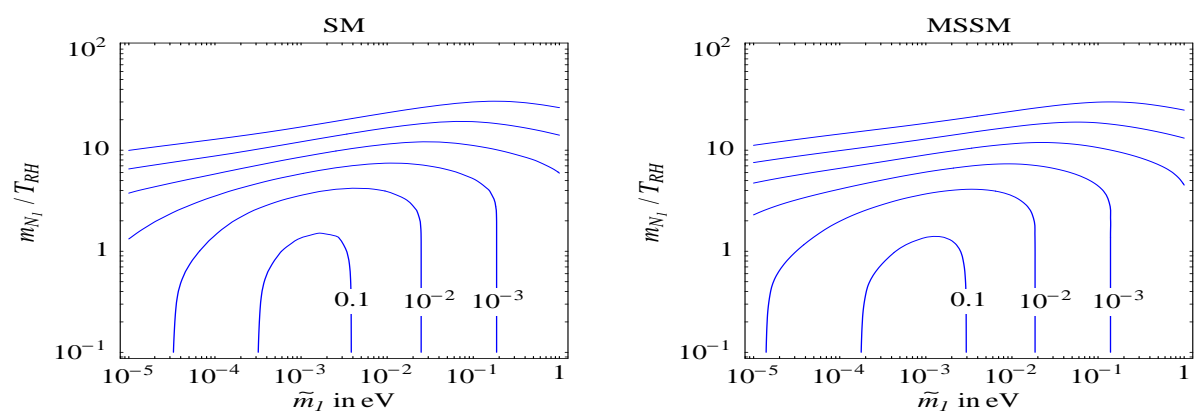

Figure 9: The efficiency parameter $\kappa$ as function of $\left(\tilde{m}_{1}, M_{R 1} / T_{\mathrm{RH}}\right)$ for the Type I Seesaw with hierarchical $N_{R}$, in the SM and MSSM. In this plot $M_{R 1}=10^{10} \mathrm{GeV}$; the plot would only change slightly for $M_{R 1} \ll 10^{14} \mathrm{GeV}$.

$\frac{\tilde{m}_{j} M_{j}^{2}}{8 \pi v_{\mathrm{wk}}^{2}}$, where $\tilde{m}_{j}$ is often of order of the elements of the $\nu_{L}$ mass matrix, although it is a rescaled $N_{R}$ decay rate. The requisite $C P$ violating decay asymmetry $\epsilon_{1}$ is caused by the interference of the tree level contribution and the one-loop corrections in the decay rate of the heavy Majorana neutrinos. For hierarchical neutrinos it is given by:

$$
\begin{aligned}
\epsilon_{1} & \equiv \frac{\Gamma\left(N_{1} \rightarrow \Phi^{-} \ell^{+}\right)-\Gamma\left(N_{1} \rightarrow \Phi^{+} \ell^{-}\right)}{\Gamma\left(N_{1} \rightarrow \Phi^{-} \ell^{+}\right)+\Gamma\left(N_{1} \rightarrow \Phi^{+} \ell^{-}\right)} \\
& \simeq-\frac{3}{16 \pi} \sum_{j \neq 1} \frac{\operatorname{Im}\left(Y_{\nu}^{\dagger} Y_{\nu}\right)_{1 j}^{2}}{\left(Y_{\nu}^{\dagger} Y_{\nu}\right)_{11}} \frac{M_{1}}{M_{j}}
\end{aligned}
$$

where $\Phi$ and $\ell$ indicate the Higgs field and the charged leptons, respectively.

Eq. (27) can be of the order of the observed $Y_{B} \sim 3 \times 10^{-11}$ when the following conditions are satisfied:

1. $M_{R 1}$ should be $\lesssim T_{R H}{ }^{9}$. This temperature is unknown, but bounded above in certain scenarios (e.g. $T_{R H} \lesssim 10^{9} \mathrm{GeV}$ due to gravitino overproduction in some supersymmetric models, see 4.2.6). This can be seen in Figure9, where the efficiency factor $\kappa$ falls off rapidly for $M_{R 1}>T_{R H}$.

2. The $N_{R 1}$ decay rate $\propto \tilde{m}_{1}$ should sit in a narrow range. To be precise, $\tilde{m}_{1}$ must be large enough to produce an approximately thermal number density of $N_{R 1}$, and small enough that the $N_{R 1}$ lifetime is of order the age of the Universe at $T \sim M_{R 1}$ (the out-of-equilibrium decay condition). These two constraints are encoded in the efficiency factor $\kappa$, plotted in Figure 9.

3. $\epsilon_{1}$ must be $\gtrsim 10^{-6}$.

In Figure 10 is plotted the baryon asymmetry, produced by thermal leptogenesis, as a function of $M_{R 1}$ and $\tilde{m}_{1}$, for $T_{R H} \gg M_{R 1}$, and $\epsilon_{1}=10^{-6}$. To reproduce the observations, $M_{R 1}$ and $\tilde{m}_{1}$ must be inside the three neighboring (blue) lines.

\footnotetext{
${ }^{9}$ In the so-called 'strong washout' regime, $T_{R H}$ can be an order of magnitude smaller than $M_{1}$ [185].
} 


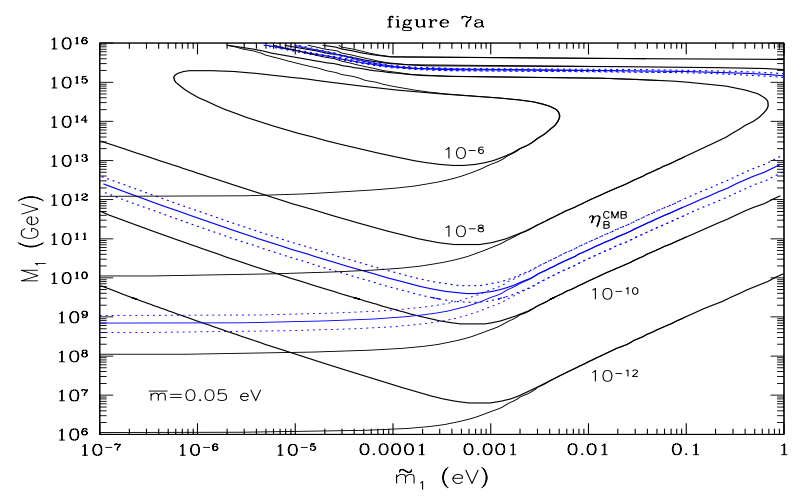

Figure 10: Contour plot, from [186], of the baryon to photon ratio produced in thermal leptogenesis, as a function of $M_{R 1}$ and $\tilde{m}_{1}$. The decay asymmetry $\epsilon_{1}$ was taken to be $10^{-6}$. The three (blue) close-together lines are the observed asymmetry. The horizontal contours, for small $\tilde{m}_{1}$ assume a thermal $N_{R}$ abundance as initial condition.

\subsubsection{Parametrizing the type I seesaw}

Twenty-one parameters are required to determine the three generation lepton sector of the type I seesaw model. This includes the charged lepton masses, and a mixing matrix (with three complex parameters, e.g. the MNSP matrix) in the left-handed lepton sector. The remaining 9 real numbers and 3 phases can be chosen in various ways:

1. "top-down" - input the $N_{R}$ sector: the eigenvalues of the mass matrix $M_{R}$ and of $Y_{\nu}$, and a matrix transforming between the bases where these matrices are diagonal [138] (see also Refs. $[187,188]$ ).

2. "bottom-up" - input the $\nu_{L}$ sector: the eigenvalues of the mass matrix $M_{\nu}^{I}$ and of $Y_{\nu}$, and a matrix transforming between the bases where these matrices are diagonal.

3. "intermediate" - the Casas-Ibarra parametrization [158]: the $M_{R}$ and $M_{\nu}^{I}$ eigenvalues, and a complex orthogonal matrix $R$ which transforms between these two bases.

To relate the RH parameters relevant for leptogenesis to the LH ones, many of which are accessible at low energy, it is useful to consider the first and second parametrization.

\subsubsection{Implications for CP conserving observables}

The second requirement (i.e., the range of $\tilde{m}_{1}$ ) sets an upper bound on the mass scale of light neutrinos. The scaled decay rate $\tilde{m}_{1}$ is usually $\sim m_{2}, m_{3}$; for hierarchical light neutrinos, it naturally sits in the desired range. One can show $[189,190]$ that $m_{1}<\tilde{m}_{1}$, so that $m_{1} \lesssim 0.15 \mathrm{eV}[183,185,191,192]$ is required for thermal leptogenesis in the type I seesaw, with hierarchical $N_{R}$. This is shown in Figure 11.

In type I seesaw models with hierarchical $N_{R}$, the third condition $\left(\epsilon_{1} \gtrsim 10^{-6}\right)$ imposes $M_{R 1} \gtrsim 10^{8} \mathrm{GeV}$, because $\epsilon_{1} \leq 3 M_{R 1}\left(m_{3}-m_{1}\right) /\left(8 \pi v_{\mathrm{wk}}^{2}\right)$ in most of parameter 

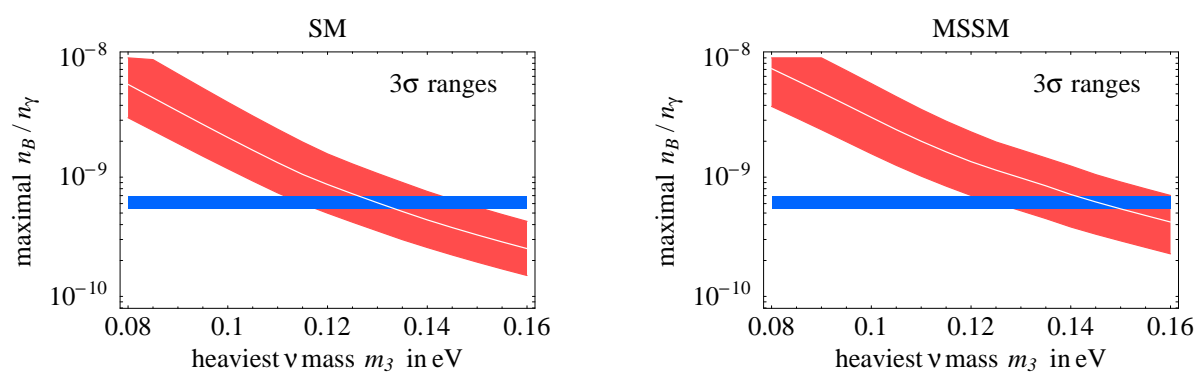

Figure 11: Upper bound on the light neutrino mass scale, assuming hierarchical $N_{R}$, taken from [192]. The plot shows the measured baryon asymmetry (horizontal line) compared with the maximal leptogenesis value as function of the heaviest neutrino mass $m_{3}$.

space $[190,192]$. For three $N_{R}$, the value of $M_{R 1}$ has little implication on low energy neutrino observables. If $\epsilon_{1}$ is maximal - that is, $M_{R 1}$ close to its lower bound, - this sets one constraint on the 21 parameters of the type I seesaw. This has no observable consequences among Standard Model particles, because at most 12 masses, angles and phases are measurable, and $\epsilon_{1}$ can be maximized by choice of the nine other parameters. The situation is more promising $[138,193]$ in SUSY models with universal soft terms, where some of the 9 additional parameters can contribute to slepton RGEs and thereby to lepton flavor violating branching ratios, as discussed in Section 4.1 .

\subsubsection{Relations between Leptogenesis and leptonic CP violation}

The leptogenesis parameter $\epsilon_{1}$ is a $C / P$ asymmetry, suggesting a possible correlation with $\mathrm{CP}$ violation in $\nu$ oscillations (the phase $\delta$ ), or to the low energy Majorana phases $\left(\phi_{1,2}\right)$. Let us assume that $\epsilon_{1}$ is large enough — so thermal leptogenesis works - and concentrate on the implications for low-energy $\mathrm{CP}$ violation.

The first thing that must always be said, in discussing potential connections between phases in the MNSP lepton mixing matrix and leptogenesis, is that there is no linear relation: leptogenesis can work when there is no $C / P$ in MNSP, and measuring low energy leptonic phases does not imply that there is $\mathrm{CP}$ violation available for leptogenesis. This was clearly and elegantly shown by Branco, Morozumi, Nobre and Rebelo in [194]. The problem is that six phases are included in the general three neutrino seesaw scenario it would be astonishing if the $C / P$ parameter we are interested in $\left(\epsilon_{1}\right)$ is proportional to the low energy phases $\left(\delta, \phi_{1,2}\right)$.

Nonetheless, some sort of relation between $\epsilon_{1}$ and the low energy phases would be interesting - so what can we say? Needing more inputs that the data provides is a familiar problem for extensions of the SM. The usual solutions are to scan over unknowns, or to fix them. But this is subtle: any relation depends on the choice of "independent" phases. For instance, if $\epsilon_{1}$ and $\delta$ are chosen as inputs, then it follows that they are unrelated ${ }^{10}$. A choice of parametrization is not obvious: the $C / P$ parameter $\epsilon_{1}$ is a function of $N_{R}$ phases, masses, mixing angles, whereas the observable phases are those of $U$.

A useful step is to write $\epsilon_{1} \propto$ a Jarlskog invariant, which can be done for thermal

\footnotetext{
${ }^{10}$ This arises in the Casas-Ibarra seesaw parametrization.
} 
leptogenesis with hierarchical $N_{R}[195]$ :

$$
\epsilon_{1} \propto \Im\left\{\operatorname{Tr}\left[M_{\nu}^{\dagger} M_{\nu} M_{\nu}^{\dagger}\left(Y_{\nu} Y_{\nu}^{\dagger}\right)^{-1} M_{\nu}\left(Y_{\nu}^{*} Y_{\nu}^{T}\right)^{-1}\right]\right\}
$$

The advantage is that Jarlskog invariants can be evaluated in any basis/parametrization. It is easy to see, evaluating Eq. (29) in the $\nu_{L}$ mass eigenstate basis, that the $C / P$ for leptogenesis is controlled by a matrix $W$ which transforms between the bases were $Y_{\nu}$ and $M_{\nu}$ are diagonal. This matrix is unobservable, verifying the no-go theorem of [194]. However, in many popular/common Yukawa texture models, where $Y_{\nu}$ and $M_{\ell}$ are almost simultaneously diagonalizable, $W \sim U$ and the MNSP phases are relevant for thermal leptogenesis. For $s_{13}$ larger than the mixing angles between diagonal $Y_{\nu}$ and $M_{\ell}$, $\epsilon_{1} \propto \sin 2\left(\phi_{1}-\phi_{2}+\delta\right)$.

\subsubsection{Model dependent approaches}

Within specific models interesting links between the phase relevant for leptogenesis and the phase $\delta$ measurable in neutrino oscillation experiments have been made. The precise link depends on how many "texture" zeroes are assumed to be present in the neutrino Dirac mass matrix. For example within the class of two right-handed neutrino models, if two texture zeroes are assumed then there is a direct link between $\delta$ and the leptogenesis phase, with the sign of $\delta$ being predicted from the fact that we are made of matter rather than antimatter $[196,197]$. Two right-handed neutrino models can be obtained as a limiting case of sequential dominance models, and in such models if only the physically motivated texture zero in the 11 entry of the Dirac mass matrix is assumed, then the link is more indirect [198]. Other approaches which give rise to a link between leptogenesis and CP violation include GUT models [199], or textures [117, 138, 187, 200] or left-right symmetric models, to be discussed in the next Subsection. On the other hand, if the charged lepton sector contributes significantly to the lepton mixing $\theta_{13}$ and therefore also to $\delta$, such links may be spoiled [201].

\subsubsection{Leptogenesis in supersymmetric scenarios}

In supersymmetric scenarios, the history of the early universe is subject to various constraints. Many of them are associated to the gravitino problem [202,203]. In short, unstable gravitinos are notoriously in conflict with nucleosynthesis (see [204] for a recent analysis), while stable ones may 'overclose' the universe. If the gravitino is very light $\left(m_{3 / 2} \lesssim \mathrm{keV}[205]\right)$ or very heavy $\left(m_{3 / 2} \gtrsim 10 \mathrm{TeV}\right)$, these bounds disappear, and thermal leptogenesis works (see, e.g., [206]). For all other masses, nucleosynthesis or 'overclosure' constraints translate into bounds on the gravitino abundance at $T \sim 1 \mathrm{MeV}$ or today, respectively. Assuming that gravitinos are not produced by inflaton decays (see [207]), this gravitino abundance is linear in the reheat temperature [208].

Unstable gravitinos with masses below $\sim 10 \mathrm{TeV}$ lead to severe constraints on the reheat temperature $T_{R H}[204]$ which are in conflict with thermal leptogenesis where $T_{R H} \gtrsim$ $10^{9} \mathrm{GeV}$. There are, however, various alternative leptogenesis scenarios such as nonthermal leptogenesis [209] where the heavy neutrinos are directly produced by inflaton decays, or mechanisms using the superpartner of the neutrino, the sneutrino [210], or 
sneutrino oscillations (see Subsec. 8.3). These scenarios can be consistent with unstable gravitinos.

Stable gravitinos, on the other hand, may evade the constraints from nucleosynthesis provided that the decays of the next-to-lightest superpartner into the gravitino are harmless [211]. However, the 'overclosure' constraint leads to $T_{R H} \lesssim\left(10^{9}-10^{10}\right) \mathrm{GeV}$. Such an upper bound on the reheat temperature is suggested independently by string-theoretical

arguments where $T_{R H} \lesssim \sqrt{m_{3 / 2} M_{\mathrm{P}}}[212]$. Stable gravitinos are thus (marginally) consistent with thermal leptogenesis, and provide a natural dark matter candidate [213]. It is clear that the neutrino mass bound, as discussed in 4.2.3, becomes much tighter now since $\widetilde{m}_{1} \sim 10^{-3} \mathrm{eV}$ (cf. Fig. [10) and $m_{1} \lesssim \widetilde{m}_{1}[189,190]$. This scenario therefore predicts hierarchical light neutrinos as well as gravitino cold dark matter. These predictions will be tested in neutrino experiments and at future colliders [214].

\subsection{Leptogenesis and type II seesaw mechanism}

In type II seesaw scenarios the neutrino mass matrix $M_{\nu}$ reads

$$
M_{\nu}^{I I}=M_{L}-M_{\nu}^{D} M_{R}^{-1}\left(M_{\nu}^{D}\right)^{T} \equiv M_{L}+M_{\nu}^{I}
$$

where we divided the mass matrix in the conventional type I part $M_{\nu}^{I}=-M_{\nu}^{D} M_{R}^{-1}\left(M_{\nu}^{D}\right)^{T}$ and the part characteristic for type II, $M_{L}$. A type II seesaw term can, e.g., be present in $S O(10)$ models, in which the $B-L$ symmetry is broken by a $\mathbf{1 2 6}$ Higgs fields. Depending on the parameters of the model, either $M_{L}$ or $M_{\nu}^{I}$ can be the dominating source of $M_{\nu}^{I I}$. As mentioned above, in case of the conventional type I seesaw mechanism with three families of light and heavy Majorana neutrinos, there are six phases. As already discussed, in this case there is in general no relation between the PMNS phase and leptogenesis phase.

For the type II case the phase counting gives the result of 12 independent CP phases and there is no connection between low and high energy $\mathrm{CP}$ violation either. The number of CP phases can be obtained by going to a basis in which both $M_{L}$ and $M_{R}$ are real and diagonal. Any $\mathrm{CP}$ violation will then stem from the matrices $M_{\nu}^{D}$ and $M_{\ell} M_{\ell}^{\dagger}$ (with $M_{\ell}$ being the charged lepton mass matrix). Those two matrices posses in total $9+3=12$ phases.

The term $M_{L}$ is induced by a $S U(2)_{L}$ Higgs triplet, whose neutral component acquires a vev $v_{L} \propto v_{\mathrm{wk}}^{2} / M_{\Delta_{L}}$, where $M_{\Delta_{L}}$ is the mass of the triplet and $v_{\mathrm{wk}}$ the weak scale. Consequently, the triplet contribution to the neutrino mass matrix is

$$
M_{L}=v_{L} f_{L}
$$

with $f_{L}$ a symmetric $3 \times 3$ coupling matrix. The magnitude of the contribution of $\Delta_{L}$ to $M_{\nu}^{I I}$ is thus characterized by its vev $v_{L}$. In left-right symmetric theories the left-right symmetry necessarily implies the presence of a $S U(2)_{R}$ triplet, whose coupling matrix is given by $f_{R}=f_{L} \equiv f$ and its vev is given by $v_{R}$, where $v_{L} v_{R}=\gamma v_{\mathrm{wk}}^{2}$ with $\gamma$ a model dependent factor of order one. The right-handed Majorana neutrino mass matrix is thus given by $M_{R}=v_{R} f=v_{R} / v_{L} M_{L}$. Before acquiring its vev, the presence of the doubly charged Higgs and the coupling of the Higgs triplet with the doublet introduces the possibility of additional diagrams capable of generating a lepton asymmetry. First, there 

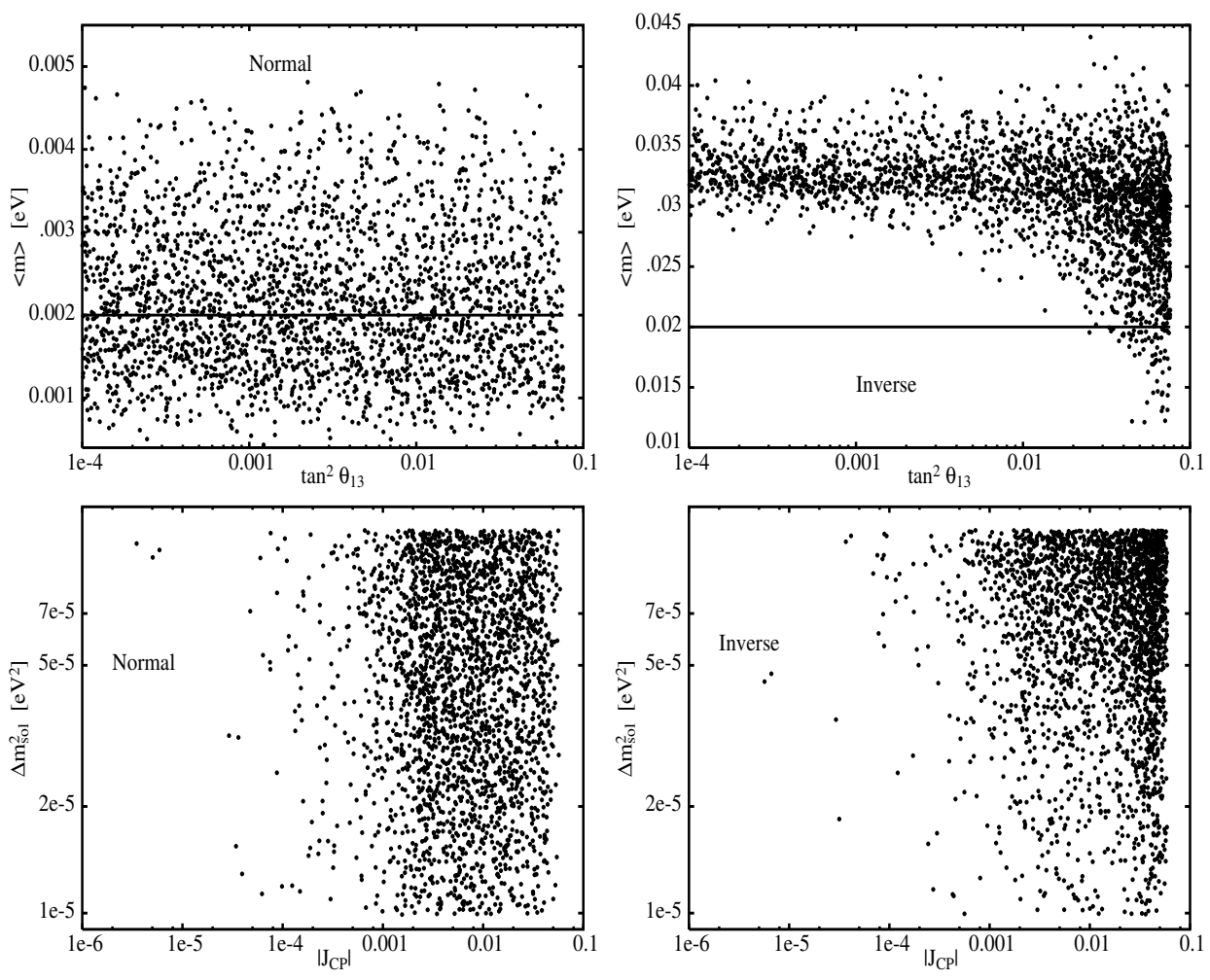

Figure 12: Scatter plots of the effective mass against $\tan \theta_{13}$ and $\Delta m_{\odot}^{2}$ against $J_{C P}$ for the normal (left) and inverted (right) neutrino mass spectrum. Taken from [220].

is the possibility that in the decay $N_{1} \rightarrow L H_{u}$ a virtual Higgs triplet is exchanged in the one-loop diagram, which contributes to the decay asymmetry in the decay of the heavy Majorana neutrinos [215-218]. The corresponding term $\epsilon_{1}^{\Delta}$ adds up to the conventional term $\epsilon_{1}$ whose properties were discussed in the previous Subsection.

The second new diagram possible is given by the decay of the doubly charged Higgs triplet in two charged leptons. One-loop exchange of a heavy Majorana neutrino gives rise to the decay asymmetry [215]

$$
\epsilon_{\Delta} \equiv \frac{\Gamma\left(\Delta_{L} \rightarrow l^{c} l^{c}\right)-\Gamma\left(\Delta_{L}^{*} \rightarrow l l\right)}{\Gamma\left(\Delta_{L} \rightarrow l^{c} l^{c}\right)+\Gamma\left(\Delta_{L}^{*} \rightarrow l l\right)}
$$

If $M_{1} \ll M_{\Delta_{L}}\left(M_{1} \gg M_{\Delta_{L}}\right)$ the decay of the Majorana neutrino (Higgs triplet) will govern the baryon asymmetry. Thus, depending on which term dominates $M_{\nu}^{I I}$, four different situations are possible [217]. The discussion of 3 of the cases has so far not been discussed in as much detail as the conventional leptogenesis in type I seesaw mechanisms.

If $M_{1} \ll M_{\Delta_{L}}$ and the conventional term $M_{\nu}^{I}$ dominates $M_{\nu}^{I I}$, we recover the usual seesaw and leptogenesis mechanisms and the statements given in Sec. 4.2.1 apply.

In situations in which $M_{1} \ll M_{\Delta_{L}}$, the heavy Majorana neutrinos display a hierarchical structure and $M_{L}$ dominates $M_{\nu}^{I I}$, it has been shown in $[217,218]$ that one can rewrite the decay asymmetries such that $\epsilon_{1}^{\Delta}$ depends on $M_{L}$ and $\epsilon_{1}$ on $M_{\nu}^{I}$. However, since matrices are involved, $\epsilon_{1}$ can still be the dominant contribution to the decay asymmetry, a situation which in the context of left-right symmetry has intensively been investigated in [219,220], 
see also [221]. Calculating in this framework the baryon asymmetry in terms of light neutrino parameters (a bottom-up approach) leads typically to a main dependence on the Majorana phases in the PMNS matrix. If $M_{\nu}^{D}$ is given by the up-quark mass matrix and the light neutrinos display a normal hierarchal spectrum, one of the low energy Majorana phases has to be very close to zero or $\pi / 2$ [219]. For $M_{\nu}^{D}$ given by the down quark or charged lepton mass matrix one finds that the in general unknown mass spectrum of the heavy Majorana neutrinos is exactly given by the measurable mass spectrum of the light Majorana neutrinos. In case of a normal hierarchy, the Majorana phases should lie around $\pi / 4$ or $5 \pi / 4$. Both values give comparable results for the rate of neutrinoless double beta decay. Thus, measuring neutrinoless double beta decay fully determines the neutrino mass matrix in this scenario. It is also possible to set limits on the lightest neutrino mass $m_{1}$ because the baryon asymmetry is proportional to $m_{1}$. It should be larger than $10^{-5}$ $\mathrm{eV}$ in order to produce a sufficient baryon asymmetry [220]. For an inverted hierarchy of the neutrinos it turns out that rather sizable values of $\theta_{13}$ are required. Thus, sizable effects of CP violation in future long-baseline neutrino oscillation experiments are possible. The preferred value of the Majorana phase implies in addition a rather sizable rate of neutrinoless double beta decay. Furthermore, the lightest neutrino mass should be heavier than $10^{-3} \mathrm{eV}$. Figure 12 shows for the normal and inverted hierarchy typical examples for the expected values of $\theta_{13}$, the effective mass and the CP violating parameter $J_{C P}$ in neutrino oscillations. A similar example within a framework incorporating spontaneous $\mathrm{CP}$ violating is discussed in Section 5.2.4.

Finally, if neutrinos possess a quasi-degenerate mass spectrum, one of the Majorana phases is required to lie around $\pi$ or $\pi / 2$. A measurement of neutrinoless double beta decay can resolve this ambiguity.

The other possible scenarios have not been discussed in detail in the literature so far (see, e.g., [222]). General statements are however possible. If, e.g., $M_{1} \ll M_{\Delta_{L}}$ and the term $\epsilon_{1}^{\Delta}$ dominates the decay asymmetry, the limits on the light neutrino masses of order $0.1 \mathrm{eV}$ (see Sec. 4.2.3) no longer apply [217], since the couplings responsible for the neutrino masses do not influence the wash-out processes. For hierarchical light neutrinos the upper bounds on the decay asymmetry $\epsilon_{1}$ and $\epsilon_{1}^{\Delta}$ are identical. In case of quasidegenerate neutrinos, however, the limit in case of type II seesaw is weaker by a factor of $2 m_{0}^{2} / \Delta m_{\mathrm{A}}^{2}$ [218], where $m_{0}$ is the common neutrino mass scale. Along the same lines, the lower limit of order $10^{9} \mathrm{GeV}$ on the lightest of the heavy Majorana neutrinos can be relaxed by roughly one order of magnitude [218], thereby making thermal leptogenesis less in conflict with the gravitino problem.

Consider the case when $M_{1} \gg M_{\Delta_{L}}$ and $M_{\nu}^{I}$ dominates $M_{\nu}^{I I}$. Then $\epsilon_{\Delta}$ will produce the baryon asymmetry and again the limits on light neutrino masses do not apply. The same is true when $M_{1} \gg M_{\Delta_{L}}$ and $M_{L}$ is the main contribution to $M_{\nu}^{I I}$. A smaller range of allowed parameters is expected in this case [217].

Therefore, given the fact that quasi-degenerate light neutrinos are hard to reconcile with standard thermal leptogenesis in type I seesaw models, our discussion implies that if we learn from future experiments that neutrinos are indeed quasi-degenerate, triplet induced leptogenesis represents a valid alternative (this was first noted in [223]).

Also possible is — in inflationary scenarios - that the decay of the inflaton into light 
particles together with interference of one-loop diagrams with exchanged $S U(2)$ triplets and heavy Majorana neutrinos generates a lepton asymmetry. Various slepton decays in future colliders are expected to be observable [224].

Up to now the discussion was constrained to the presence of only one triplet. If only one triplet is present, right-handed Majorana neutrinos are necessary to produce a decay asymmetry. Introducing two or more triplets allows self-energy diagrams which can produce a decay asymmetry without heavy Majorana neutrinos [225]. The possibility of lowering the triplet mass scale due to a resonance effect of close-in-mass triplets is possible [226], giving the prospect of collider phenomenology. The presence of light and detectable Majorons is also possible. There are models implementing this kind of triplet self-energy scenarios with light left-handed neutrinos [226] and with quasi-degenerate ones [227]. The latter also predicts a stable proton due to $R$ parity conservation. Introducing the triplet induced neutrino mass matrix of the type II seesaw mechanism along the lines of [228], i.e., by a conjunction of flavor and permutation symmetries will typically include many additional Higgs fields. Rich phenomenology in form of rare charged lepton decays or charged lepton EDMs will be among the interesting consequences.

To sum up, the type II seesaw mechanism displays the most general but more complicated framework of neutrino mass and leptogenesis. Nevertheless, richer phenomenology is expected, most of which remains to be explored.

\subsection{Dirac Leptogenesis}

Since the seesaw mechanism coupled with existing data on neutrino masses and mixings does not give complete information about the RH neutrino sector, one must consider leptogenesis within various scenarios for $\mathrm{RH}$ neutrino masses that correctly explain neutrino observations. In this section, we discuss a possibility that the leptogenesis occurs with Dirac neutrinos. The conventional leptogenesis [172], which we call Majorana leptogenesis for definiteness, was based on the fact that the standard model violates $B+L$ [173], while the Majorana neutrinos violate $L$, and hence both $B$ and $L$ are violated. Therefore it is possible to create $L$ from the decay of right-handed neutrino that is subsequently converted to $B$ [184]. On the other hand, Dirac neutrinos conserve $L$ and hence $B-L$ is an exact symmetry. Therefore $B-L$ stays vanishing throughout the evolution of the universe and it appears impossible to generate non-vanishing baryon asymmetry ${ }^{11}$.

Dirac leptogenesis overcomes this problem by the following simple observation [229]. Recall that the Dirac neutrinos have tiny Yukawa couplings, $M_{\nu}^{D}=Y_{\nu} v_{\mathrm{wk}}, Y_{\nu} \simeq 10^{-13}$. If this is the only interaction of the right-handed neutrinos, thermalization is possible only by processes like $N L \rightarrow H W$ and they do not thermalize for $T \gtrsim g^{2} Y_{\nu}^{2} M_{\mathrm{Pl}} \sim 10 \mathrm{eV} \cdot{ }^{12}$ At this low temperature, obviously both $H$ and $W$ cannot be produced and the thermalization is further delayed until $T_{\nu} \simeq M_{\nu}$ when neutrinos become non-relativistic. Therefore the

\footnotetext{
${ }^{11}$ An obvious exception is electroweak baryogenesis, where $B-L=0$ while $B=L \neq$ after the electroweak phase transition.

${ }^{12}$ In contrast, the Yukawa coupling of the right-handed electron $e_{R}$ is large enough to equilibrate the $e_{R} \mathrm{~s}$ before sphaleronic processes switch off [230].
} 


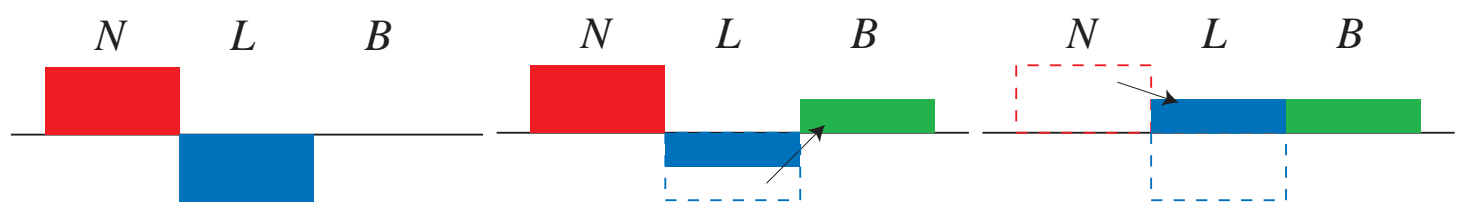

Figure 13: The evolution of the lepton asymmetry in Dirac leptogenesis models. At the first stage, an asymmetry between the ordinary leptons and the right-handed neutrinos is created without lepton-number violation. Then the asymmetry in the ordinary leptons is partially converted to the baryon asymmetry. Finally, the right-handed neutrinos come in thermal equilibrium with the other leptons. The net baryon and lepton asymmetries remain while the overall $B-L$ vanishes.

number of left-handed and right-handed neutrinos are separately conserved practically up to now. We call them $L$ and $N$, respectively, and the total lepton number is $L+N$. The combination $L+N-B$ is strictly conserved.

Suppose the decay of a heavy particle produced an asymmetry $L=-N \neq 0$. The overall lepton number is conserved (see Fig. 13). $N$ is frozen down to $T_{\nu}$. On the other hand, the lepton asymmetry $L$ is partially converted to the baryon asymmetry via the Standard Model anomaly. After the electroweak phase transition $T \lesssim 250 \mathrm{GeV}$, the anomaly is no longer effective. Finally at $T_{\nu}, L$ and $N$ equilibrate. In the end there is a baryon asymmetry $B=-(L+N)$.

The original paper [229] introduced new electroweak doublet scalar $\Phi$ that has the same quantum numbers as the Higgs doublets and Yukawa couplings $\Phi L N$ and $\Phi^{*} L E$. If there are two sets of them, there is CP violation and their decays can create the asymmetry $L=-N \neq 0$. However, these doublets are there just for this purpose and have no other motivations.

On the other hand, light Dirac neutrinos are natural in models where the neutrino Yukawa couplings are tied to the small supersymmetry breaking effects [231,232]. The Dirac neutrino mass is due to the effective operator

$$
\int d^{2} \theta \frac{\chi}{M} L H_{u} N
$$

where $\chi$ is the hidden sector field which acquires a vacuum expectation value $\langle\chi\rangle \simeq$ $m_{3 / 2}+\theta^{2} m_{3 / 2} M_{\mathrm{Pl}}$ and $M$ is the heavy mass scale. The neutrino Yukawa coupling is $Y_{\nu} \simeq m_{3 / 2} / M$, and is naturally small. The operator can be obtained by integrating out (two sets of) new doublets $\phi+\phi^{c}$ that couple as $W=\phi N H_{u}+\phi^{c} L \chi+M \phi \phi^{c}$. The asymmetries $L=-N \neq 0$ are created by the decay of $\phi$ [233]. Then the origin of small neutrino mass and the origin of the lepton asymmetry are tied in the same way as the Majorana leptogenesis. Also concerning the gravitino problem (cf. 4.2.6), Dirac and Majorana leptogenesis are on the same footing [233,234]. Such a scenario may be supported by the lack of neutrinoless double beta decay as well as the existence or righthanded sneutrino at LHC and Linear Collider. 


\subsection{Resonant Leptogenesis}

The right handed neutrino sector of generic seesaw models is almost entirely unconstrained by existing data on neutrino masses and mixings. It is therefore necessary to consider all the various possibilities for the structure of the $\mathrm{RH}$ neutrino sector which are compatible with current experimental data. In this section, we consider the case where two or more right-handed neutrinos are nearly degenerate in mass.

An important, further motivation for this possibility comes from the severe limits on the right handed neutrino sector that exist in models of thermal leptogenesis with hierarchical right handed neutrinos. In particular there exists a bound on the mass of the lightest right handed neutrino, $M_{R 1} \lesssim T_{R H}$, discussed in section 4.2.1. In supersymmetric theories with unstable gravitinos, this bound can be in conflict with the bound $T_{R H} \lesssim 10^{9} \mathrm{GeV}$, coming from nucleosynthesis considerations (see 4.2.6). This motivates us to consider scenarios where the scale of the right handed neutrino masses can be lowered whilst still being compatible with thermal leptogenesis [175, 176, 239]. This may be achieved naturally in scenarios with nearly degenerate right handed neutrinos [175], in complete accordance with current neutrino data, and with the advantage that the final baryon asymmetry generated is independent of the initial lepton, baryon or heavy neutrino abundances [177].

If the mass difference between two heavy Majorana neutrinos happens to be much smaller than their masses, the self-energy ( $\epsilon$-type) contribution to the leptonic asymmetry becomes larger than the corresponding ( $\epsilon^{\prime}$-type) contribution from vertex effects [174, 175]. Resonant leptogenesis can occur when this mass difference of two heavy Majorana neutrinos is of the order of their decay widths, in which case the leptonic asymmetry could be even of order one $[175,177]$. As a result, one can maintain the RH neutrino masses around the GUT scale [242] or one can contemplate the possibility that the heavy neutrino mass scale relevant to thermal leptogenesis is significantly lower, for example in the TeV range [175]. This of course requires a different realization of the seesaw mechanism [90] but it can be in complete accordance with the current neutrino data [177].

The magnitude of the $\epsilon$-type $\mathrm{CP}$ violation occurring in the decay of a heavy Majorana neutrino $N_{i}$ is given by [175],

$$
\epsilon_{N_{i}}=\frac{\operatorname{Im}\left(Y_{\nu}^{\dagger} Y_{\nu}\right)_{i j}^{2}}{\left(Y_{\nu}^{\dagger} Y_{\nu}\right)_{i i}\left(Y_{\nu}^{\dagger} Y_{\nu}\right)_{j j}} \frac{\left(m_{N_{i}}^{2}-m_{N_{j}}^{2}\right) m_{N_{i}} \Gamma_{N_{j}}^{(0)}}{\left(m_{N_{i}}^{2}-m_{N_{j}}^{2}\right)^{2}+m_{N_{i}}^{2} \Gamma_{N_{j}}^{(0) 2}}
$$

where $\Gamma_{N_{i}}^{(0)}$ is the tree level total decay width of $N_{i}$. It is apparent that the CP asymmetry will be enhanced, possibly to $\epsilon \sim 1$, provided

$$
\begin{gathered}
m_{N_{2}}-m_{N_{1}} \sim \frac{1}{2} \Gamma_{N_{1,2}}^{(0)}, \\
\frac{\operatorname{Im}\left(Y_{\nu}^{\dagger} Y_{\nu}\right)_{i j}^{2}}{\left(Y_{\nu}^{\dagger} Y_{\nu}\right)_{i i}\left(Y_{\nu}^{\dagger} Y_{\nu}\right)_{j j}} \sim 1 .
\end{gathered}
$$

It is important to note that Eq. (34) is only valid for the mixing of two heavy Majorana neutrinos. Its generalization to the three neutrino mixing case is more involved and is given in [177]. 


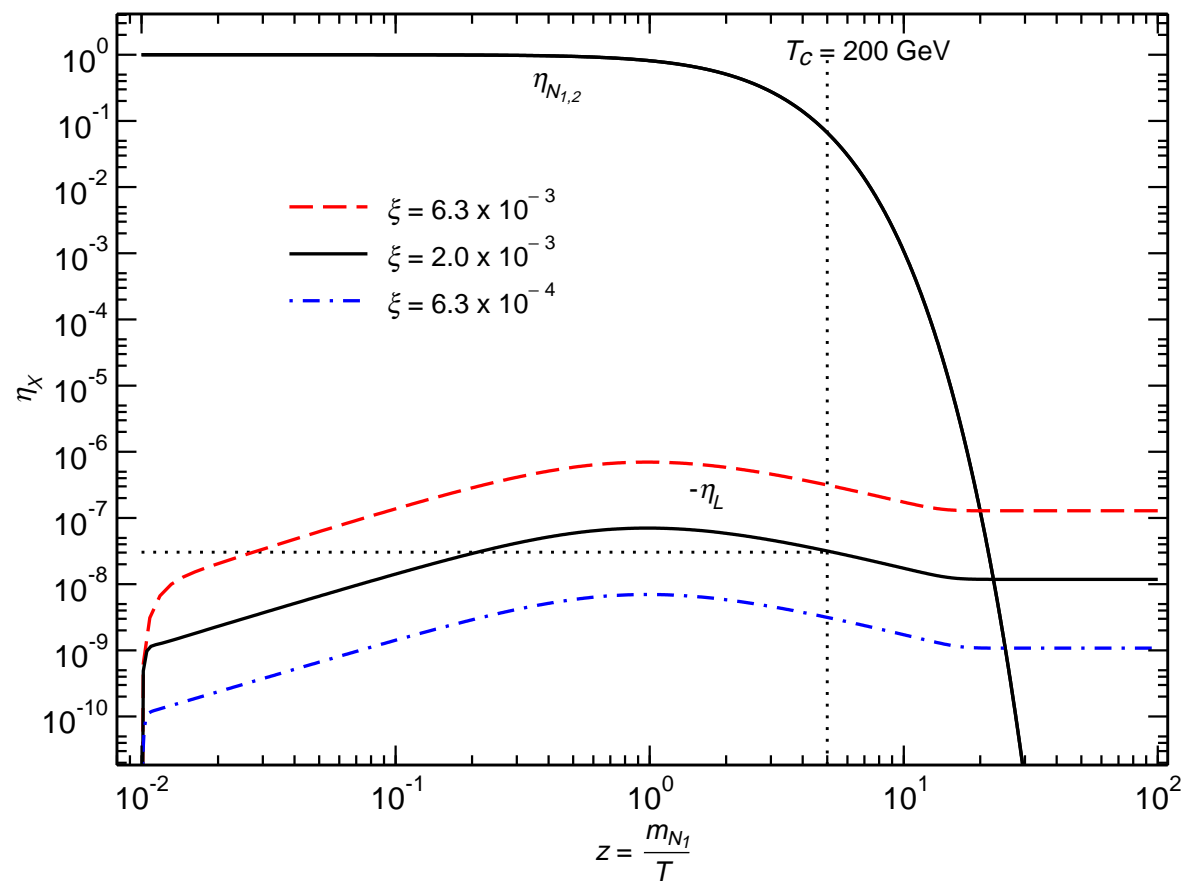

Figure 14: Numerical estimates of the lepton to photon, and neutrino to photon ratios, $\eta_{L}$ and $\eta_{N_{1,2}}$ as functions of $z=m_{N_{1}} / T$ for scenarios with $m_{N_{1}}=1 \mathrm{TeV}$. The model is based on the Froggatt-Nielsen mechanism and is completely consistent with all current neutrino data (see [177] for details). It naturally provides a degeneracy of $\frac{m_{N_{2}}}{m_{N_{1}}}-1=9.2 \times 10^{-11}$, with $K$-factors and CP asymmetries of $K_{1}=K_{2}=6570$ and $\delta_{N_{1}}=\delta_{N_{2}}=-0.003$. $\xi$ is a free parameter. The horizontal dotted line shows the value of $\eta_{L}$ needed to produce the observed $\eta_{B}$. The vertical dotted line corresponds to $T=T_{c}=200 \mathrm{GeV}$.

Successful leptogenesis requires conditions out of thermal equilibrium. To quantify this, we introduce the parameter, $K_{N_{i}}^{l}=\Gamma_{N_{i}}^{l} / H\left(T=m_{N_{i}}\right)$ where $H(T)$ is the Hubble parameter and $\Gamma_{N_{i}}^{l}$ is the total decay width of $N_{i}$ into a lepton species $l(l=e, \mu, \tau)$. In typical hierarchical leptogenesis scenarios $K_{N_{i}}^{l}$ is small, usually $K_{N_{i}}^{l} \sim 1$. This constraint can be translated directly into an upper bound on the Yukawa couplings of the neutrinos which can be expressed in terms of effective light neutrino masses, $\widetilde{m}_{i}$,

$$
\widetilde{m}_{i} \equiv \frac{v^{2}\left(Y_{\nu}^{\dagger} Y_{\nu}\right)_{i i}}{2 m_{N_{i}}} \simeq 10^{-3} K_{N_{i}} \mathrm{eV}
$$

where $K_{N_{i}}=\sum_{l} K_{N_{i}}^{l}$. However, resonant leptogenesis can be successful with values of $K_{N_{i}}$ larger than 1000 [177] (see Figure 14). This has implications for leptogenesis bounds on the absolute mass scale of the light neutrinos. If a large, $\gtrsim 0.2 \mathrm{eV}$, Majorana mass was seen in neutrinoless double beta decay, this could be naturally accommodated with resonant leptogenesis, whereas thermal leptogenesis models based on hierarchical heavy neutrinos would be strongly disfavored, as they naturally require smaller values of $K_{N_{i}}$ [186,235].

Conditions close to thermal equilibrium (with large $K_{N_{i}}$ ) endow resonant leptogenesis models with another particularly attractive feature; the final baryon asymmetry generated is almost independent of the initial baryon, lepton or heavy neutrino abundances $[177,179]$. 
For $K_{N_{i}} \gtrsim 1$, and under the assumption that the neutrino Yukawa couplings are the same for each lepton flavour, an order of magnitude estimate for the baryon to photon ratio may be obtained by [177]

$$
\eta_{B}^{\text {univ. }} \sim-10^{-2} \times \sum_{N_{i}} e^{-\left(m_{N_{i}}-m_{N_{1}}\right) / m_{N_{1}}} \frac{\delta_{N_{i}}}{K}
$$

where $\delta_{N_{i}}$ is the leptonic CP asymmetry in the decay of $N_{i}, K=\sum_{l} K_{l}$ and

$$
K_{l}=\sum_{N_{i}} e^{-\left(m_{N_{i}}-m_{N_{1}}\right) / m_{N_{1}}} K_{N_{i}}^{l}
$$

It is apparent that if the CP-asymmetry is enhanced, for example through resonant effects, then $K$ can be increased without an impact on the final baryon asymmetry.

In resonant leptogenesis scenarios with neutrino Yukawa couplings that are not universal for each lepton flavour, the effects of individual lepton flavours on the resultant baryon asymmetry may become very important $[178,179]$. This also applies to scenarios with mildly hierarchical RH neutrinos [179]. These effects may result in an enhancement of the baryon asymmetry predicted using (37) by a factor as large as $10^{6}$ in some models of resonant leptogenesis. An order of magnitude estimate for the final baryon asymmetry, when the neutrino Yukawa couplings are not flavour-universal, may be obtained by [179]

$$
\eta_{B} \sim-10^{-2} \times \sum_{l=1}^{3} \sum_{N_{i}} e^{-\left(m_{N_{i}}-m_{N_{1}}\right) / m_{N_{1}}} \delta_{N_{i}}^{l} \frac{K_{N_{i}}^{l}}{K_{l} K_{N_{i}}}
$$

where $\delta_{N_{i}}^{l}$ is the CP asymmetry in the decay of $N_{i}$ to leptons of flavour $l$. For a more precise computation of the resultant baryon asymmetry, a network of Boltzmann equations must be solved, one for each heavy Majorana neutrino species and one for each lepton flavour [179]. By adding a further Boltzmann equation for the baryon abundance, and including effects due to the rate of $B+L$ violating sphaleron transitions, it can be shown that successful resonant leptogenesis is possible with heavy Majorana neutrinos as light as the electroweak scale [179].

In particular, models of resonant $\tau$-leptogenesis [178], where a lepton asymmetry is generated predominantly in the $\tau$-family, can allow large Yukawa couplings between the $e$ and $\mu$ lepton families and some of the $\mathrm{RH}$ neutrinos. These couplings, in conjunction with the low RH neutrino scale, lead to a significant amount of accessible phenomenology, such as potentially observable neutrinoless double beta decay, $\mu \rightarrow e \gamma, \mu \rightarrow$ eee and coherent $\mu \rightarrow e$ conversion in nuclei, and the possibility of the collider production of heavy Majorana neutrinos [178, 179].

The conditions for resonant leptogenesis can be met in several ways. Models based on the Froggatt-Nielsen mechanism can naturally provide nearly degenerate heavy Majorana neutrinos satisfying Eq. (35) and provide a light neutrino spectrum fulfilling all experimental constraints. It can be shown that a model like this can produce the observed baryon asymmetry by solving the network of Boltzmann equations - including gauge mediated scattering effects [177] (see Figure 14). In this model the 'heavy' Majorana neutrinos can be as light as $1 \mathrm{TeV}$. 
$S O(10)$ models with a "type III seesaw mechanism" naturally predict pairs of nearly degenerate heavy Majorana neutrinos suitable for resonant leptogenesis [236]. In addition, a model of neutrino mass from SUSY breaking has been shown to naturally lead to conditions suitable for resonant leptogenesis [238].

In the radiative leptogenesis mechanism [181], small mass differences arise through renormalization group corrections between $\mathrm{RH}$ neutrinos which are exactly degenerate in mass at some high scale. The leptonic CP asymmetry induced in this scenario is sufficient to produce the observed baryon asymmetry.

In soft leptogenesis [180,237], soft SUSY breaking terms lead to small mass differences between sneutrinos. Resonant effects allow sneutrino decay to generate the required CP asymmetry.

Several other mechanisms for leptogenesis where the right handed neutrinos can be at a $\mathrm{TeV}$ scale have been suggested [239]. Clearly for the seesaw mechanism to operate in such models, the Dirac mass must be constrained, e.g., by a leptonic global U(1) symmetry [240]. 


\section{The Heavy Majorana Mass Matrix}

\subsection{General Considerations}

We have already seen from the Introduction that the type I seesaw mechanism requires the existence of right-handed neutrinos $N_{R}$, and then the light Majorana mass matrix is given as

$$
M_{\nu}=-M_{\nu}^{D} M_{R}^{-1} M_{\nu}^{D^{T}}
$$

where $M_{\nu}^{D}$ is the Dirac neutrino matrix (to be thought of as perhaps similar to the quark and charged lepton mass matrices) and $M_{R}$ is the heavy Majorana mass matrix. While the elements of $M_{\nu}^{D}$ must be at or below the electroweak scale, the characteristic scale of right-handed neutrino masses can and must be much higher. Having introduced righthanded neutrinos into the Standard Model for the purpose of accounting for light physical neutrino masses via the type I seesaw mechanism ${ }^{13}$ it is clearly an important question to understand the mass spectrum and couplings of the right-handed neutrinos. Since their only couplings are their Yukawa couplings to Higgs and left-handed neutrino fields, it will clearly not be an easy task to answer this question. However there are three areas where important clues may emerge: the light-neutrino mass matrix $M_{\nu}$; the baryon asymmetry of the universe; and (assuming supersymmetry) lepton flavor violation. Taken together with other theoretical ideas, we shall show that it may be possible to shed light on the right-handed neutrino sector of the theory.

\subsubsection{The Three Right-Handed Neutrino Paradigm}

It is most common to assume that there are exactly three right-handed neutrinos. Such an assumption is motivated by unified theories such as $S O(10)$ which predicts that the number of right-handed neutrinos is equal to the number of quark and lepton families, since a single right-handed neutrino makes up each 16-plet of the theory. In fact this prediction also follows more generally from any theory which contains a gauged righthanded group $S U(2)_{R}$, such as left-right symmetric theories, Pati-Salam and so on.

Assuming three right-handed neutrinos one can ask whether their mass spectrum is hierarchical, or contains an approximate two or three-fold degeneracy. From the point of view of the type I seesaw mechanism in Eq. (40) it is clear that the answer to this question depends on the nature of the Dirac neutrino mass matrix $M_{\nu}^{D}$. For example, suppose that the right-handed neutrinos had a three-fold degeneracy $M_{R}=\operatorname{diag}(M, M, M)$, then the Eq. (40) would predict

$$
M_{\nu}=-\frac{M_{\nu}^{D} M_{\nu}^{D^{T}}}{M} .
$$

Then if the Dirac neutrino mass matrix were hierarchical and approximately proportional to the up-type quarks, for example, then Eq. (41) would imply

$$
m_{1}: m_{2}: m_{3} \approx m_{u}^{2}: m_{c}^{2}: m_{t}^{2}
$$

\footnotetext{
${ }^{13}$ Here we shall assume $M_{\nu}=M_{\nu}^{\mathrm{I}}$. We shall later comment also on the type II seesaw mechanism.
} 
which is much too strong a mass hierarchy compared to the rather mild experimentally measured ratio $0.1 \leq m_{2} / m_{3} \leq 1$.

The remaining two possibilities are that the three right-handed neutrinos are either hierarchical or contain an approximate two-fold degeneracy. In either case it is convenient to work in a basis where their mass matrix is diagonal:

$$
M_{R}=\left(\begin{array}{ccc}
X^{\prime} & 0 & 0 \\
0 & X & 0 \\
0 & 0 & Y
\end{array}\right)
$$

The neutrino Dirac mass matrix $M_{\nu}^{D}$ in this basis can be written as

$$
M_{\nu}^{D}=\left(\begin{array}{lll}
a^{\prime} & a & d \\
b^{\prime} & b & e \\
c^{\prime} & c & f
\end{array}\right)
$$

where in this convention the first column of Eq. (44) couples to the first right-handed neutrino, the second column of Eq. (44) couples to the second right-handed neutrino and so on. Note that in the hierarchical case in Eq. (43) we do not specify which of the three right-handed neutrinos $X^{\prime}, X, Y$ is the lightest one, which is the intermediate one and which is the heaviest one, since the columns of $M_{\nu}^{D}$ and the eigenvalues $X^{\prime}, X, Y$ of $M_{R}$ may simultaneously be re-ordered without changing $M_{\nu}$.

Having displayed the unknown Yukawa couplings associated with the Dirac neutrino mass matrix in Eq. (44) it is clear that without further input it is not possible to say anything about the right-handed neutrino masses or couplings from the experimentally determined light Majorana mass matrix $M_{\nu}$. On the other hand, rather natural theoretical assumptions can lead to a great deal of information about the unknown masses and couplings of the right-handed neutrinos, as we now discuss.

Regarding the implementation of the type I seesaw mechanism there seem to be two possible options: either all the right-handed neutrinos contribute equally (democratically) to each element of $M_{\nu}$, or some right-handed neutrinos contribute more strongly than others. In the second case, called right-handed neutrino dominance [241], a rather natural implementation of the seesaw mechanism is possible. For example if the right-handed neutrino of mass $Y$ contributes dominantly to the physical neutrino mass $m_{3}$, and the right-handed neutrino of mass $X$ contributes dominantly to the physical neutrino mass $m_{2}$, while the right-handed neutrino of mass $X^{\prime}$ contributes dominantly to the physical neutrino mass $m_{1}$, then a sequential dominance of these three contributions leads to a neutrino mass hierarchy $m_{1} \ll m_{2} \ll m_{3}$. With such sequential dominance the mixing angles are then given as simple ratios of Dirac neutrino mass matrix elements: $\tan \theta_{23} \approx$ $e / f, \tan \theta_{12} \approx \sqrt{2} a /(b-c)$, which can be naturally large independently of the neutrino mass hierarchy.

The physical neutrino masses are given by $m_{3} \approx\left(e^{2}+f^{2}\right) / Y, m_{2} \approx 4 a^{2} / X, m_{1} \approx$ $\left(a^{\prime}, b^{\prime}, c^{\prime}\right)^{2} / X^{\prime}$ and the mass ordering of the right-handed neutrino masses $X^{\prime}, X, Y$ is not determined unless further information is specified about the Dirac neutrino masses. In 
general there are six possible mass orderings of the three right-handed neutrinos:

$$
\begin{aligned}
& Y<X<X^{\prime} \\
& Y<X^{\prime}<X \\
& X<Y<X^{\prime} \\
& X^{\prime}<Y<X \\
& X^{\prime}<X<Y \\
& X<X^{\prime}<Y
\end{aligned}
$$

The dominant right-handed neutrino of mass $Y$ (the one mainly responsible for the mass $m_{3}$ ) may thus be the lightest one as in Eqs. (45, 46), the intermediate one as in Eqs. (47. 48), or the heaviest one as in Eqs. (49, 501). The neutrino of mass $X^{\prime}$ is essentially irrelevant from the point of view of the light Majorana mass matrix $M_{\nu}$, since the lightest physical neutrino of mass $m_{1}$ is approximately zero in the hierarchical case. Thus $X^{\prime}$ cannot be determined from any low energy experiments such as neutrino oscillations or neutrinoless double beta decay. If $X^{\prime}$ happens to be the heaviest right-handed neutrino, as in Eqs. (45) 47) then if its mass is above the GUT scale then it completely decouples from observable physics. In this case the three right-handed neutrino model becomes effectively a two right-handed neutrino model [241]. However even in this case there is a remaining ambiguity about whether the dominant right-handed neutrino is the lightest one or the next-to-lightest one as in Eqs. (45, 477).

\subsubsection{Grand Unification}

It is clear that further theoretical input is required in order to elucidate the nature of the masses and couplings of the right-handed neutrinos. In some GUT model, one can expect generically that the Dirac neutrino masses are related to the other quark and charged lepton masses and this additional information about $M_{\nu}^{D}$ can then be input into the type I seesaw formula Eq. (40) to help to yield information about the right-handed neutrino mass matrix $M_{R}$. For example assuming that $M_{\nu}^{D} \approx M^{u}$, the up-type quark mass matrix, and inputting the approximately determined light Majorana mass matrix, the seesaw formula can then be rearranged to yield right-handed neutrino masses with a very hierarchical mass spectrum [242]:

$$
M_{1}: M_{2}: M_{3} \approx m_{u}^{2}: m_{c}^{2}: m_{t}^{2},
$$

which can be compared to the naive expectation for the physical neutrino masses in Eq. (42). Numerically Eq. (51) yields the order of magnitude estimates $M_{1} \sim 10^{5} \mathrm{GeV}$, $M_{2} \sim 10^{10} \mathrm{GeV}, M_{1} \sim 10^{15} \mathrm{GeV}$, with an uncertainty of a least one or two orders of magnitude in each case. In addition there may be special cases which completely invalidate these estimates.

In specific GUT models the above expectations can also be very badly violated. For example in the $S O(10)$ model with $S U(3)$ family symmetry [243], although the neutrino Dirac mass matrix is strikingly similar to the up-type quark mass matrix, a very different pattern of right-handed neutrino masses emerges:

$$
M_{1}: M_{2}: M_{3} \approx \epsilon^{6} \bar{\epsilon}^{3}: \epsilon^{6} \bar{\epsilon}^{2}: 1
$$


where $\epsilon \approx 0.05, \bar{\epsilon} \approx 0.15$. In this model the dominant right-handed neutrino is the lightest one $Y=M_{1}$, with $X=M_{2}$, while the heaviest right-handed neutrino is decoupled $X^{\prime}=M_{3}$ as in Eq. (45). This model therefore acts effectively as a two right-handed neutrino model, with the two right-handed neutrinos being very similar in mass, with interesting implications for leptogenesis, to which we now turn.

\subsubsection{Leptogenesis}

Leptogenesis and lepton flavor violation are important indicators which can help to resolve the ambiguity of right-handed neutrino masses in Eqs. (45) [50). In the simplest case of two right-handed neutrino models, leptogenesis has been well studied with some interesting results [196-198]. In general, successful thermal leptogenesis for such models requires the mass of the lightest right-handed neutrino model to be quite high, and generally to exceed the gravitino constraints, required if supersymmetry is assumed. Such a strong bound is also at odds with the strong right-handed neutrino mass hierarchy expected from GUTs as in Eqs. (51, 52). In unified theories with type II see-saw, this potential problem can be resolved (see e.g. [244]).

In three right-handed neutrino models with sequential dominance, if the dominant right-handed neutrino is the lightest one, then the washout parameter $\tilde{m}_{1} \sim \mathcal{O}\left(m_{3}\right)$ is rather too large compared to the optimal value of around $10^{-3} \mathrm{eV}$ for thermal leptogenesis. However, if the dominant right-handed neutrino is either the intermediate or the heaviest one then one finds $\tilde{m}_{1} \sim \mathcal{O}\left(m_{2}\right)$ or arbitrary $\tilde{m}_{1}$, which can be closer to the desired value [245].

\subsubsection{Sneutrino Inflation}

It has been suggested that a right-handed sneutrino, the superpartner to a right-handed neutrino, could be a candidate for the inflaton in theories of cosmological inflation. This has interesting consequences for the masses and couplings of the right-handed neutrinos. For example in the case of chaotic sneutrino inflation, the mass of the right-handed sneutrino inflaton must be about $10^{13} \mathrm{GeV}$ [246], while in sneutrino hybrid inflation its mass could be considerably lighter [247]. In both scenarios, the decaying sneutrino inflaton can be responsible for non-thermal leptogenesis, and can give a reheat temperature compatible with gravitino constraints providing its Yukawa couplings are sufficiently small. This typically implies that the associated right-handed neutrino must be effectively decoupled from the see-saw mechanism, so that it corresponds to the decoupled right-handed neutrino of mass $X^{\prime}$ in sequential dominance discussed above.

\subsubsection{Type II Seesaw Models}

Once the more general type II seesaw framework is permitted [73], then it apparently becomes more problematic to determine the properties of the right-handed neutrinos which contribute to $M_{\nu}$ via the type I part of the seesaw mechanism. On the other hand, the type II seesaw mechanism provides the most direct way of raising the neutrino mass scale to a level that will be observable in neutrinoless double beta decay. Furthermore, the difficulties of providing consistency of leptogenesis scenarios with the gravitino bound in 
supersymmetric theories, or simply with the strong right-handed neutrino mass hierarchy expected from GUT models, motivates a more general type II seesaw framework which can in principle help resolve some of these difficulties. It has recently been shown $[104,248]$ how to construct natural models for partially degenerate neutrinos by using an $S O(3)$ family symmetry to add a type II contribution to the light neutrino Majorana mass matrix proportional to the unit matrix, with large neutrino mixing originating from sequential dominance. Compared to the pure type I limit, the masses of the right-handed neutrinos become larger if the mass scale of the light neutrinos is increased via the type II contribution. This can also help to resolve the potential conflict between the typical predictions for $M_{1}$ as in Eqs. $(47,48)$ and thermal leptogenesis [244]. In addition, increasing the neutrino mass scale has interesting phenomenological consequences, such as a decreasing CP violating phase $\delta$ and a decreasing mixing angle $\theta_{13}$, testable in future experiments.

\subsubsection{Right-Handed Neutrinos in Extended Technicolor}

In the mechanism that has been constructed for producing light neutrinos in extended Technicolor $[92,93,441,442]$, there are two right-handed neutrinos. Interestingly, this mechanism involves a seesaw, but one in which the relevant Dirac neutrino mass matrix elements are greatly suppressed down to the level of a few $\mathrm{keV}$, and the Majorana masses are also suppressed, of order $100 \mathrm{MeV}$ to $1 \mathrm{GeV}$. The origin of this suppression stems from the fact that the left- and right-handed chiral components of neutrinos transform differently under the ETC gauge group. Although the mechanism does involve a seesaw, it does not involve any GUT-scale masses. This is clear, since extended Technicolor models do not contain any such mass scales. It serves as an existence proof of how a seesaw mechanism can work with much lower Dirac and Majorana mass scales than the usual GUT-scale seesaw.

\subsection{Seesaw Neutrino mass and Grand unification}

One of the major ideas for physics beyond the Standard Model is supersymmetric grand unification (SUSY GUT) [249]. It is stimulated by a number of observations that are in accord with the general expectations from SUSY GUTs : (i) A solution to the gauge hierarchy problem i.e why $v_{\text {wk }} \ll M_{\mathrm{Pl}}$; (ii) unification of electroweak, i.e. $S U(2)_{L} \times$ $U(1)_{Y}$ and strong $S U(3)_{c}$ gauge couplings assuming supersymmetry breaking masses are in the $\mathrm{TeV}$ range, as would be required by the solution to the gauge hierarchy; (iii) a natural way to understand the origin of electroweak symmetry breaking. Supersymmetric grand unified theories generically predict proton decay via dimension-five operators, which typically give large branching ratios for modes like $p \rightarrow \bar{\nu}_{\mu} K^{+}$. The current lower limits on proton decay modes place significant constraints on these theories and probably rule out a number of simpler SUSY GUT models [250]. Nevertheless, the idea of grand unification is so attractive that we will proceed on the basis that appropriate modifications allow supersymmetric GUTs to evade current nucleon decay limits.

Gauge coupling unification leads to a unification scale of about $10^{16} \mathrm{GeV}$ and simple seesaw intuition leads to a seesaw scale of $10^{15} \mathrm{GeV}$ to fit atmospheric neutrino data. This suggests that seesaw scale could be the GUT scale itself; thus the smallness of neutrino 
mass could go quite well with the idea of supersymmetric grand unification. However, in contrast with the items (i) through (iii) listed above, the abundance of information for neutrinos makes it a highly nontrivial exercise to see whether the neutrino mixings indeed fit well into SUSY GUTs. In turn, the freedom in constructing realistic GUT models allows many different ways to explain current neutrino observations. Thus even though neutrino mass is a solid evidence for physics beyond the Standard Model, the true nature of this physics still remains obscure. The hope is that the next round of the experiments will help to narrow the field of candidate theories a great deal.

To see how this is likely to come about, the first point is the choice of the grand unification group. Even though attempts to implement the seesaw mechanism using an extension of the $\mathrm{SU}(5)$ with the addition of a right-handed neutrinos have been made, a more natural GUT gauge group from the point of view of neutrino mass is $\mathrm{SO}(10)$ since its basic spinor representation contains the right-handed neutrino automatically along with the other fifteen fermions of the Standard Model (for each family). Thus in some sense one could argue that small neutrino masses have already chosen $S O(10)$ GUT as the most natural way to proceed beyond the Standard Model. $S O(10)$ has therefore rightly been the focus of many attempts to understand neutrino mixings. It turns out that within the $S O(10)$ SUSY GUTs there are many ways to understand large mixings. We outline below only the major differences among the different ideas. The hope is that they differ in their predictions sufficiently so that they can be tested by planned experiments.

One of the features that distinguishes $S O(10)$ from $\mathrm{SU}(5)$ is the presence of local $B-L$ symmetry as a subgroup and the $S O(10)$ models divide into two classes depending on whether $B-L$ symmetry is broken by a 16 Higgs field or an $\mathbf{1 2 6}$. In the first case the right-handed neutrino mass necessarily arises out a nonrenormalizable coupling whereas in the second case it arises from a renormalizable one. Secondly, the breaking of $B-L$ by 16 Higgs necessarily leads to low energy MSSM with R-parity breaking so that the model cannot have cold dark matter without additional assumptions, whereas $\mathbf{1 2 6}$ breaking of $B-L$ preserves R-parity at low energies so that the low energy MSSM that derives from such an $S O(10)$ has a natural dark matter candidate i.e. the lightest SUSY particle.

As noted in the Introduction, the $S O(10)$ model has in general the type II seesaw formula for neutrino masses which can reduce to type I for some range of parameters. For instance, in the $\mathbf{1 6}$ based models, the first term in type II seesaw formula is negligible and therefore the neutrino masses are dictated by type I seesaw formula. In contrast in 126 Higgs models, the neutrino mass can be given either by the first term or the second term in the type II seesaw formula or both.

\subsubsection{A minimal 126-based $S O(10)$ model}

As mentioned, in $S O(10)$ models where a 126 Higgs breaks $B-L$ symmetry, the righthanded neutrino masses can arise from renormalizable couplings. A minimal model of this type based on a single $\mathbf{1 0}$ and a single $\mathbf{1 2 6}$ field has a number of attractive features [251, 252]. Since the $\mathbf{1 2 6}$ field also contributes to charged fermion masses through the MSSM doublets in it, this model unifies the flavor structure in the quark and the neutrino sector thereby increasing the predictivity of the model in the neutrino sector. In fact in the absence of $\mathrm{CP}$ violation, the model has only 12 free parameters all of which are 


\begin{tabular}{|c||c|}
\hline Model & $\theta_{13}$ \\
\hline 126 based models & \\
Goh, Mohapatra, Ng & 0.18 \\
Chen, Mahanthappa & 0.15 \\
\hline $\mathbf{1 6}$ based models & \\
Babu, Pati, Wilczek & 0.0005 \\
Albright, Barr & 0.014 \\
Ross, Velasco-Sevilla & 0.07 \\
Blazek, Raby, Tobe & 0.05 \\
\hline
\end{tabular}

Table 6: The table lists some typical predictions for $\theta_{13}$ in different $S O(10)$ models and shows how the next generation of experiments can narrow the field of possible $\mathrm{SO}(10)$ unification models.

determined by the quark masses and mixings along with the charged lepton masses. As a result all mixings and masses are predicted by the model up to an overall scale. It has been shown that if one uses the type I seesaw formula, the model fails to reproduce the observed solar mixing angle and also the solar mass difference squared and is therefore ruled out [253]. It has however been shown recently that if one uses the type II seesaw mechanism with the first term dominating the neutrino mass matrix, the large mixings come out due to b-tau mass convergence in a very natural manner [254]. In particular, an interesting prediction of this model is that $\theta_{13} \simeq 0.18$ making it quite accessible to the next generation of experiments such as long-baseline, off-axis and the reactor experiments.

\subsubsection{6-based models}

The main characteristic of the $S O(10)$ models where a 16 Higgs breaks $B-L$ is that righthanded neutrino masses arise from nonrenormalizable couplings in the superpotential, the implicit assumption being that there is a high-scale theory (perhaps string theory or a renormalizable high scale theory with heavier fields) that below the heavy scale leads to this version of $S O(10)$. This means that without additional symmetry restriction, there are more parameters than the physical inputs. Often in these models symmetries that tend to explain quark mixings restrict the number of couplings somewhat and one can make predictions in the neutrino sector. There exist several interesting examples of this kind of models [255]. Several of these models tend to give values for $\theta_{13}$ which are much below the range that can be probed by the next generation of planned experiments. We give a very small sample of the different predictions for $\theta_{13}$ in models with both $\mathbf{1 6}$ and 126 in Table 6 .

\subsubsection{Summary of what we can learn about $S O(10)$}

A review on different neutrino mass models based on $S O(10)$ can be found in Ref. [256]. From these models, we learn that

1. First a very generic prediction of all $S O(10)$ models is that the neutrino mass hierarchy is normal. The basic reason for this is the quark lepton symmetry inherent in the model, which tends to make the neutrino Dirac mass to be of similar hierarchy as the quarks, which via seesaw mechanism implies normal hierarchy for neutrinos. 
leading to $\Delta m_{23}^{2} \geq 0$. Again this is a result that can be probed in the long-baseline oscillation or neutrinoless double beta decay experiments;

2. The second point about the $S O(10)$ models is that they make definite predictions about the mixing angle $\theta_{13}$ as given in Table [6] and often for the other mixing angles. The planned experiments will therefore considerably narrow the field of viable $S O(10)$ models through their measurement or upper limit on these mixing parameters.

\subsubsection{Implications of Models with Spontaneous CP Violation}

Relations between leptogenesis and CP violation in low energy processes generally do not exist due to the presence of un-known mixing angles and phases in the heavy neutrino sector, as discussed in Sec. 4.2.4. In models with spontaneous CP violation, all Yukawa coupling constants are real. CP violation occurs due to the presence of the phases in the expectation values of the scalar fields, which break the gauge symmetry spontaneously. Recently it has been shown that [257] in the minimal left-right $S U(2)_{L} \times S U(2)_{R}$ symmetric model [72] with spontaneous CP violation, there exist very pronounced relations between the $\mathrm{CP}$ violation in low energy processes, such as neutrino oscillation and neutrinoless double beta decay, and leptogenesis, which occurs at a very high energy scale. The minimal left-right symmetric model contains a bi-doublet and a pair of triplet Higgses. Using the gauge degrees of freedom, one can rotate away all but two phases present in the expectation values of the scalar fields. Thus there are only two intrinsic phases, the relative phase between the two vevs in the bi-doublet and that between the left- and right-handed triplets, to account for all $\mathrm{CP}$ violation in the quark sector and in the lepton sector. The relative phase between the two vevs in the bi-doublet is responsible for CP violation observed in the quark sector, while $\mathrm{CP}$ violation in the lepton sector dominantly comes from the relative phase between the vevs of the two triplet Higgses. The relative phase between the two vevs in the bi-doublet appears in the lepton sector only at the sub-leading order due to the large hierarchy in the bi-doublet vevs required by a realistic quark sector. As a result, the relation between $\mathrm{CP}$ violation in the quark sector and $\mathrm{CP}$ violation in the lepton sector is rather weak. Due to the left-right parity, the $\mathrm{RH}$ and LH neutrino Majorana mass terms are proportional to each other, which further reduces the unknown parameters in the model. In this model, both leptogenesis and the leptonic Jarlskog invariant are proportional to the sine of the relative phase between the vevs of the two triplet Higgses. Using the experimentally measured neutrino oscillation parameters as inputs, to obtain sufficient amount of lepton number asymmetry, the leptonic Jarlskog invariant has to be larger than $\sim 10^{-5}$. As the Type-II seesaw mechanism is at work, the hierarchy in the heavy neutrino sector required to obtain the observed neutrino oscillation parameters is very small, leading to a heavier mass for the lightest RH neutrino, compared to the case utilizing the Type-I seesaw mechanism. As a result, the requirement that the decay of the lightest $\mathrm{RH}$ neutrino is out-of-equilibrium in thermal leptogenesis can be easily satisfied.

Similar attempts have been made to induce spontaneous CP violation from a single source. In one such attempt SM is extended by a singlet scalar field which develops a complex VEV which breaks CP symmetry [258]. Another attempt assumes that there is 
one complex VEV of the field which breaks the $B-L$ symmetry in $\mathrm{SO}(10)$ [259]. Unlike in the minimal left-right symmetry model described above [257], there is no compelling reason why all other vevs have to be real in these models.

\subsection{Renormalization group evolution of neutrino parameters}

Neutrino masses and mixing parameters are subject to the renormalization group (RG) evolution or running, i.e. they depend on energy. As theoretical predictions for these quantities typically arise from models at high energy scales such as the GUT scale, this implies that in general RG corrections have to be included in the testing of model predictions. In the case of leptonic mixing angles and $\mathrm{CP}$ phases the changes can be large for partially or nearly degenerate neutrino masses. On the other hand, strongly hierarchical masses bring about very small RG corrections for the mixing angles, while the running of the mass squared differences is sizable even in this case.

\subsubsection{Running masses, mixings and CP phases below the seesaw scale}

At energies below the seesaw scale $M_{\mathrm{R}}$, the masses of the light neutrinos can be described in a rather model-independent way by an effective dimension 5 operator if they are Majorana particles. The RG equation of this operator in the SM and MSSM [80, 260-263] leads to differential equations for the energy dependence of the mass eigenvalues, mixing angles and CP phases [264-268]. Up to $\mathcal{O}\left(\theta_{13}\right)$ corrections, the evolution of the mixing angles is given by [266]

$$
\begin{aligned}
& \dot{\theta}_{12}=-\frac{C y_{\tau}^{2}}{32 \pi^{2}} \sin 2 \theta_{12} s_{23}^{2} \frac{\left|m_{1} e^{i \varphi_{1}}+m_{2} e^{i \varphi_{2}}\right|^{2}}{\Delta m_{\odot}^{2}}, \\
& \dot{\theta}_{13}=\frac{C y_{\tau}^{2}}{32 \pi^{2}} \sin 2 \theta_{12} \sin 2 \theta_{23} \frac{m_{3}}{\Delta m_{\mathrm{A}}^{2}(1+\zeta)} \times I\left(m_{i}, \varphi_{i}, \delta\right), \\
& \dot{\theta}_{23}=-\frac{C y_{\tau}^{2}}{32 \pi^{2}} \sin 2 \theta_{23} \frac{1}{\Delta m_{\mathrm{A}}^{2}}\left[c_{12}^{2}\left|m_{2} e^{i \varphi_{2}}+m_{3}\right|^{2}+s_{12}^{2} \frac{\left|m_{1} e^{i \varphi_{1}}+m_{3}\right|^{2}}{1+\zeta}\right],
\end{aligned}
$$

where the dot indicates the differentiation $d / d t=\mu d / d \mu$ ( $\mu$ being the renormalization scale), $s_{i j}:=\sin \theta_{i j}, c_{i j}:=\cos \theta_{i j}, \zeta:=\Delta m_{\odot}^{2} / \Delta m_{\mathrm{A}}^{2}, C=-3 / 2$ in the SM and $C=1$ in the MSSM, and $I\left(m_{i}, \varphi_{i}, \delta\right):=\left[m_{1} \cos \left(\varphi_{1}-\delta\right)-(1+\zeta) m_{2} \cos \left(\varphi_{2}-\delta\right)-\zeta m_{3} \cos \delta\right] . y_{\tau}$ denotes the $\tau$ Yukawa coupling, and one can safely neglect the contributions coming from the electron and muon. For the matrix $K$ containing the Majorana phases, we use the convention $K=\operatorname{diag}\left(e^{-i \varphi_{1} / 2}, e^{-i \varphi_{2} / 2}, 1\right)$ here. For a discussion of RG effects in the case of exactly degenerate neutrino masses, where the above expressions cannot be applied, see e.g. [269-277]. ¿From Eqs. (53) one can easily understand the typical size of RG effects as well as some basic properties. First, in the SM and in the MSSM with small $\tan \beta$, the RG evolution of the mixing angles is negligible due to the smallness of the $\tau$ Yukawa coupling. Next, the RG evolution of the angles is the stronger the more degenerate the mass spectrum is. For a strong normal mass hierarchy, it is negligible even in the MSSM with a large $\tan \beta$, but for an inverted hierarchy a significant running is possible even if the lightest neutrino is massless $[278,279]$. Furthermore, non-zero phases $\delta, \varphi_{1}$ and $\varphi_{2}$ 
can either damp or enhance the running. For instance, the running of $\theta_{12}$ can be damped by non-zero Majorana phases $[81,280,281]$. Typically, $\theta_{12}$ undergoes the strongest RG evolution because the solar mass squared difference is much smaller than the atmospheric one. Finally, in the MSSM, $\theta_{12}$ runs from smaller values at high energies to larger values at low energies [282].

The RG equations for the CP phases [265-268] show that their changes are proportional to $1 / \Delta m_{\odot}^{2}$. Therefore, whenever the mixing angles run sizably, the same happens for the CP phases. This is very important for the relation between the phases relevant for high-energy processes like leptogenesis and those appearing in neutrino oscillations and neutrinoless double beta decay. The evolution of the Dirac CP phase can be especially drastic for a small CHOOZ angle, since $\dot{\delta}$ contains a term proportional to $1 / \theta_{13}$. It is also possible to generate a non-zero value of this phase radiatively if at least one of the Majorana phases is non-zero [265]. An exception is the CP conserving case where all phases are 0 or $\pi$ and do not change with energy.

Finally, the neutrino masses always change significantly with energy due to flavor-blind terms in the RG equations which contain large quantities like gauge couplings and the top Yukawa coupling. For strongly hierarchical masses and small $\tan \beta$, these terms dominate, so that the masses experience a common rescaling which is virtually independent of the mixing parameters [281].

Radiative corrections for Dirac neutrino masses have also been studied [283, 284]. Roughly speaking, the RGEs for the Dirac case are obtained from Eqs. (153) by averaging over the Majorana phases.

\subsubsection{Details of the running in seesaw models}

In order to obtain precise results, one has to go beyond the simple approximations listed above and solve the RG equations numerically. This involves solving a rather complex system of coupled differential equations, as all parameters of the theory have to be evolved from high to low energy.

A further complication arises in seesaw models with heavy singlet neutrinos which are in general non-degenerate in mass. The running above their mass thresholds is typically at least as important as the evolution below both in the SM and in the MSSM unless the neutrino Yukawa couplings are tiny [82,270,271,285-289]. This part of the RG evolution depends on many parameters of the model. An analytic understanding has been obtained only recently [290,291]. If the singlet masses are non-degenerate, one can calculate the evolution of the neutrino mass parameters by considering a series of effective theories arising from integrating out the singlets successively at the respective thresholds $[285,286]$. In general, it is not a good approximation to integrate out all singlets at the same energy scale, since the threshold corrections can be very large.

\subsubsection{Implications for model building}

As discussed above, predictions of high-energy mass models can differ substantially from low-energy experimental results due to the running. Therefore, RG corrections have to be included in the analysis. The RG evolution also opens up new interesting possibilities for model building, like the radiative magnification of mixing angles. In particular, small 
or vanishing solar mixing at high energy can be magnified to the observed large mixing at low energy (see e.g. $[82,84,292,293]$ ). Vice versa, the large but non-maximal solar mixing $\theta_{12}$ can also be reached starting from bimaximal mixing at the GUT scale $[287,294,295]$ (for examples, see Fig. 15). It is, however, important to stress that large mixing is no fixed
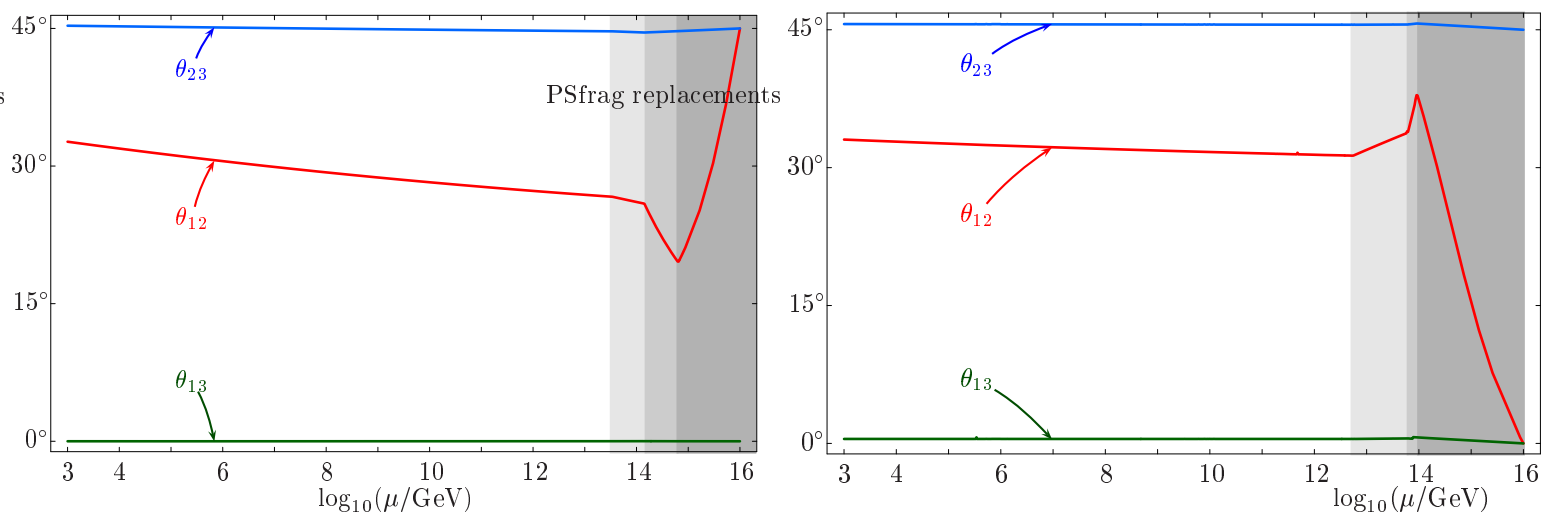

Figure 15: Examples for the RG evolution of the lepton mixing angles from the GUT scale to the SUSY-breaking scale (taken to be $\approx 1 \mathrm{TeV}$ ) in the MSSM extended by 3 heavy singlets (right-handed neutrinos) [82,287]. The masses of the lightest neutrinos for these examples are around $0.05 \mathrm{eV}$. The figures illustrate how the large but non-maximal value of the solar mixing angle $\theta_{12}$ is reached by RG running if one starts with bimaximal lepton mixing or with vanishing solar mixing at the GUT scale. The kinks in the plots correspond to the mass thresholds at the seesaw scales, where the heavy singlets are successively integrated out. The gray-shaded regions mark the various effective theories between the seesaw scales.

point under the RGE in the usual see-saw framework. It has been observed that in SUSY models large mixing can be a fixed point for different (i.e. non-seesaw) types of neutrino mass operators $[296,297]$. In addition, the small neutrino mass squared differences can be produced from exactly degenerate neutrino masses at high energy (see e.g. [275-277,298$300]$ ), if the neutrino masses are nearly degenerate. For further specific models where the $\mathrm{RG}$ evolution is relevant for neutrino masses and mixings see, for example, [248,267,301304].

\subsubsection{High-scale mixing unification and large mixings}

Another question one can ask is whether starting with small mixing angles at the seesaw scale (as would be naively expected in models with quark-lepton unification) can one get large mixings at the weak scale due to $\mathrm{RG}$ extrapolation. In a specific model where neutrino masses are quasi-degenerate, starting with neutrino mixings that are equal to quark mixings at the GUT scale i.e. $\theta_{12} \simeq V_{u s}, \theta_{23} \simeq V_{c b}$ and $\theta_{13} \simeq V_{u b}$, one can indeed get mixing angles at the weak scale which are consistent with present observations as shown in Fig. 16] below. We have chosen $\tan \beta=55$ in this calculation. An interesting point is that this mechanism works only if the common mass of the neutrinos is bigger than $0.1 \mathrm{eV}$, a prediction which can easily be tested in the proposed neutrinoless double beta decay experiments [81, 83,305-307]. 


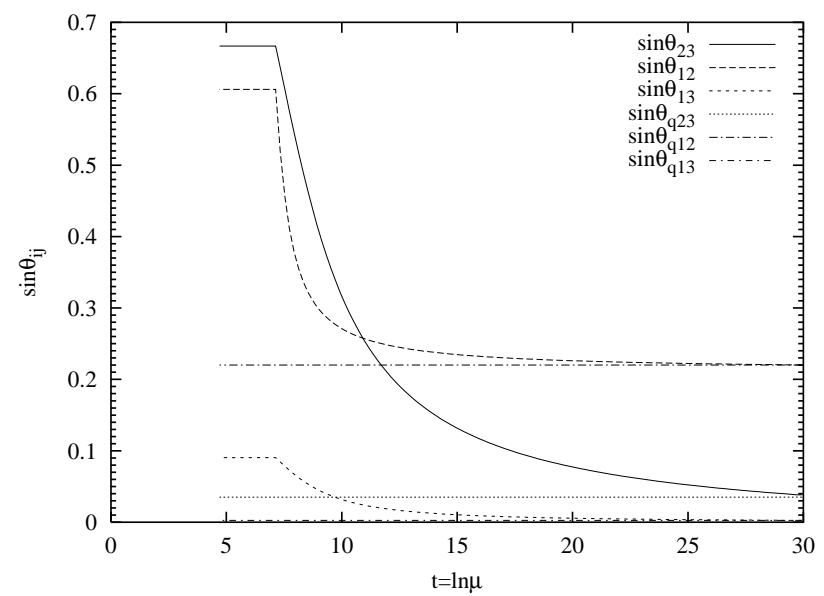

Figure 16: Evolution of small quark-like mixings at the seesaw scale to bi-large neutrino mixings at low energies for the seesaw scale $M_{R}=10^{13} \mathrm{GeV}$ with $\tan \beta=55, M_{\mathrm{SUSY}}=1$ $\mathrm{TeV}$. The solid, long-dashed and short-dashed lines represent $\sin \theta_{23}, \sin \theta_{13}$, and $\sin \theta_{12}$, respectively. The evolution of the sines of quark mixing angles, $\sin \theta_{q i j}(i, j=1,2,3)$, is presented by almost horizontal lines.

\subsubsection{Deviations of $\theta_{13}$ from 0 and of $\theta_{23}$ from $\pi / 4$ due to RG effects}

At present, observations are compatible with $\theta_{13}=0$ and $\theta_{23}=\pi / 4$. New experiments are being planned to lower the limits on deviations from these values. Additional motivation for this kind of measurements is provided by the RG: although it is possible that a symmetry produces an exactly vanishing $\theta_{13}$ and exactly maximal atmospheric mixing, this symmetry would typically operate at a high scale, and therefore its predictions would be subject to the RG evolution. Hence, without fine-tuning, one expects non-zero values of $\theta_{13}$ and $\theta_{23}-\pi / 4$ at low energy [266,277,288,308]. For example, in the MSSM one finds a shift $\Delta \sin ^{2} 2 \theta_{13}>0.01$ for a considerable parameter range, i.e. one would expect to measure a finite value of $\theta_{13}$ already in the next generation of experiments [266]. On the other hand, there are special configurations of the parameters, especially the phases, where RG effects are suppressed. Furthermore, there may be symmetries which stabilize some mixing angle completely against radiative corrections. For instance, for an inverted hierarchy with $m_{3}=0, \theta_{13}=0$ is stable under the RG [309] (see also Eqs. (53)). Hence, if future precision measurements do not find $\theta_{13}$ and $\theta_{23}-\pi / 4$ of the size of the generic $\mathrm{RG}$ change, one can restrict parameters, or even obtain evidence for a new symmetry.

\subsubsection{Implications for leptogenesis}

As has been discussed in Sec. 4.2, the requirement of successful baryogenesis via leptogenesis places an upper bound on the mass of the light neutrinos. It is important to note that in order to relate constraints on the neutrino mass spectrum coming from physics at $M_{1}$ to observation, one has to take into account radiative corrections. It turns out that there are two effects operating in opposite directions $[183,185,266,310]$ which partially cancel each other: since the mass scale is increasing, the washout driven by Yukawa couplings is stronger. On the other hand, larger $\Delta m^{2}$ s allow for a larger decay asymmetry. Taking 
into account all these effects, one finds that the upper bound on the neutrino mass scale becomes more restrictive $[183,185,266]$. The RG evolution, together with thermal effects or spectator processes [311], gives rise to the most important corrections to the mass bound [185].

\section{Non-Standard Neutrino Interactions}

\subsection{Neutrino magnetic moments}

Once neutrinos are massive, they can have transition magnetic and electric magnetic dipole moments and, in the Dirac case, also diagonal magnetic and electric dipole moments [48, 49], [40], [51]- [59]. The lepton-number conserving (diagonal and transition) magnetic and electric dipole moment operators are given by $(1 / 2) \bar{\nu}_{i} \sigma^{\mu \nu} \nu_{j} F_{\mu \nu}$ and $(-i / 2) \bar{\nu}_{i} \sigma^{\mu \nu} \gamma_{5} \nu_{j} F_{\mu \nu}$. Analogous $\Delta L=2$ expressions hold (with $i \neq j$ because of their antisymmetry under $i \leftrightarrow j$ ) for Majorana neutrinos. Therefore, a magnetic or electric dipole moment always connects one species of neutrino with another. These moments are defined for mass eigenstates. In the case of a Dirac mass eigenstate, one sees that the operator connects a left-handed electroweak-doublet neutrino to a right-handed electroweaksinglet (sterile) neutrino. In the case of Majorana mass eigenstates, the (transition) dipole moment operators connect two neutrino fields of the same chirality. The two have fundamentally different physical implications. We have reviewed some basic properties of these magnetic and electric dipole moments above.

Neutrino magnetic moments can be directly measured in terrestrial experiments using the neutrino beam from the Sun as in Super-K [312] or with neutrinos from close by nuclear reactors as in the MUNU [313] and in the Texono [314] experiments. These experiments have put upper bounds of the order of $10^{-10} \mu_{B}$ on the effective neutrino magnetic moment (defined below). It is interesting that in models involving right-handed charged currents the diagonal and transition neutrino magnetic moments $[40,48-51,53]$ are not suppressed by the neutrino mass (as in models with just the Standard Model interactions [52]) and could thus be somewhat larger. However, in general, the same interactions that can enhance neutrino magnetic moments can give corrections enhancing neutrino masses, so in a particular model one must be careful to avoid excessive loop contributions to the latter. The Borexino prototype detector has recently been utilized to put a bound of $\left|\mu_{e f f}\right|_{M S W}<5.5 \times 10^{-10} \mu_{B}$ at $90 \%$ C.L. using the elastic scattering of electrons by the solar $p p$ and ${ }^{7} B e$ neutrinos [315]. At these sub-MeV energies, the solar neutrino beam contains roughly equal proportion of $\nu_{1}$ and $\nu_{2}\left(P_{1}=P_{2}=0.5\right)$. As a result, for the same $\nu_{\text {eff }}$ the bounds on $\mu_{11}, \mu_{13}$ would be much better in experiments utilizing the $p p,{ }^{7} \mathrm{Be}$ neutrinos compared to the bounds that can be obtained by using the ${ }^{8} B$ neutrinos (which are predominantly $\nu_{2}$ ).

Neutrinos with non-zero magnetic moments contribute to the elastic scattering of electrons in water Cerenkov detectors [316-318]. The effective neutrino magnetic moment $\mu_{\text {eff }}$ (we neglect the contribution of the electric dipole moment here) responsible for the scattering event $\nu_{i}+e^{-} \rightarrow \nu_{j}+e^{-}$is proportional to the incoherent sum of outgoing 
neutrino states $\nu_{j}$ as follows:

$$
\left|\mu_{e f f}\right|^{2}=\sum_{j}\left|\sum_{i} A_{i}(L) \mu_{i j}\right|^{2}
$$

where $A_{i}(L)$ is the probability amplitude of a neutrino produced as a flavor eigenstate (lets say $\nu_{e}$ or $\bar{\nu}_{e}$ ) to be in the $i^{\prime}$ th mass eigenstate on propagating over the source-detector distance $L$. For vacuum oscillations $A_{i}(L)=U_{e i} \exp \left(-i E_{i} L\right)$ and the effective magnetic moment depends upon the $\Delta m^{2}$ and the mixing angles as follows

$$
\begin{aligned}
\left|\mu_{e f f}\right|_{V O}^{2} & =c_{12}^{2}\left(\mu_{11}^{2}+\mu_{12}^{2}+\mu_{13}^{2}\right)+s_{12}^{2}\left(\mu_{21}^{2}+\mu_{22}^{2}+\mu_{23}^{2}\right) \\
& +2 c_{12} s_{12}\left(\mu_{11} \mu_{21}+\mu_{12} \mu_{22}+\mu_{13} \mu_{23}\right) \cos \left(\frac{\Delta m_{12}^{2} L}{2 E_{\nu}}\right)
\end{aligned}
$$

In the expression for $\left|\mu_{e f f}\right|_{V O}^{2}$ above we have assumed that $U_{e 3}$ is negligibly small and the atmospheric mixing angle is maximal $\left(s_{23}^{2}=1 / 2\right)$. We have also dropped CP violating phases. In MUNU [313] and Texono [314], $\bar{\nu}_{e}$ from nuclear reactors were detected by the elastic scattering with $e^{-}$. The source-detector distance is small ( $L=18 \mathrm{~m}$ in MUNU and $L=28 \mathrm{~m}$ in TEXONO) compared to the $\nu_{1}-\nu_{2}$ oscillation length so that the Cosine term in (55) is unity. The magnetic moment matrix $\mu_{i j}$ is symmetric for Dirac neutrinos and anti-symmetric if the neutrinos are Majorana. It is clear from (55) that there exists a possibility that there may be a cancellation between the last (interference) term which could be negative and the first two terms. So experimental upper bounds on $\left|\mu_{e f f}\right|_{V O}$ which is $0.9 \times 10^{-10} \mu_{B}$ (MUNU) [313] and $1.3 \times 10^{-10} \mu_{B}$ (Texono) (both at $90 \%$ C.L.) do not constrain the elements of the $\mu_{i j}$ matrix without making added assumptions that there is no cancellation between the different terms in (5.5).

This problem does not arise for solar neutrinos as the interference term averages to zero since $2 E_{\nu} / \Delta m_{12}^{2}<<L_{\text {earth-sun. }}$. For the solar neutrinos the expression for $\left|\mu_{e f f}\right|^{2}$ reduces to a sum of two positive definite quantities

$$
\left|\mu_{e f f}\right|_{M S W}^{2}=P_{1}\left(\mu_{11}^{2}+\mu_{12}^{2}+\mu_{13}^{2}\right)+P_{2}\left(\mu_{21}^{2}+\mu_{22}^{2}+\mu_{23}^{2}\right)
$$

where $P_{1}=\left|A_{e 1}(L)\right|^{2}$ and $P_{2}=\left|A_{e 2}(L)\right|^{2}=1-P_{1}$ are the probabilities of the solar neutrinos to be in the mass eigenstate $\nu_{1}$ and $\nu_{2}$ respectively at the earth. The recent upper bound $\left|\mu_{e f f}\right|_{M S W}<1.1 \times 10^{-10} \mu_{B}$ at $90 \%$ C.L. established by Super-Kamiokande [312] can be translated into bounds on individual elements of $\mu_{i j}$ without extra assumptions. The ${ }^{8} B$ neutrinos which are detected by electron scattering at Super-K are predominantly $\nu_{2}$ state $\left(P_{2}=0.94\right.$ and $\left.P_{1}=0.06\right)$. The Super-K bound on $\left|\mu_{e f f}\right|_{M S W}$ therefore implies $\left|\mu_{12}\right|<1.1 \times 10^{-10} \mu_{B} ;\left|\mu_{22}\right|,\left|\mu_{23}\right|<1.13 \times 10^{-10} \mu_{B}$ and $\left|\mu_{11}\right|,\left|\mu_{13}\right|<4.49 \times 10^{-10} \mu_{B}$.

It is also possible to put bounds on $\mu_{i j}$ from SNO-NC data using the fact that neutrinos with non-zero magnetic moments can dissociate deuterium [319] in addition to the weak neutral currents. The bounds established from SNO-NC data do not depend upon the oscillation parameters unlike in the case of Super-K. However the bounds are poorer due to the large uncertainty in our theoretical knowledge of the theoretical ${ }^{8} B$ flux from the sun $[320]$.

We will see in a subsequent section (the one on extra dimensions) that the effective magnetic moment of the neutrinos can get substantially enhanced in a certain class of 
extra dimensions models. Searching for $\mu_{\nu}$ can therefore be used to put limits on theories with extra dimensions.

\subsection{Flavor changing and conserving nonstandard neutral cur- rent interactions}

The latest results of neutrino oscillation experiments indicate that the conversion mechanism between different neutrino flavors is driven by a non-vanishing mass difference between mass eigenstates together with large mixing angles between families. These analyses are done supposing that no non-standard neutrino interactions (NSNI) are present. In the presence of electroweak-doublet and electroweak-singlet neutrinos, the neutral weak current is, in general, nondiagonal in mass eigenstate neutrino fields [40,41]. This is the same type of nondiagonality in the neutral weak current that was present in the original Weinberg electroweak model before the advent of the Glashow-Iliopoulos-Maiani (GIM) mechanism. It will be recalled that in this original Weinberg model the $d$ and $s$ quarks were assigned to a left-handed $\mathrm{SU}(2)_{L}$ doublet $\left(u, d \cos \theta_{C}+s \sin \theta_{C}\right)_{L}$ and a left-handed $\mathrm{SU}(2)_{L}$ singlet $-d \sin \theta_{C}+s \sin \theta_{C}$. The necessary condition for the diagonality of the neutral weak current is that all of the fermions of a given charge and chirality must transform according to the same weak $T$ and $T_{3}$. Alternatively, NSNI in the form of nondiagonal couplings between different neutrino flavor eigenstates [321] and/or neutrino flavor-diagonal but flavor nonsymmetric couplings, can exist [322]. Including NSNI can modify the characteristics of neutrino conversion, and in general large values of NSNI parameters worsen the quality of the fit to data. We summarize here the present limits that can be obtained to NSNI parameters, using the result of neutrino oscillation experiments.

\subsubsection{Atmospheric neutrinos}

As repeatedly mentioned, atmospheric neutrino data are well described by the oscillation driven by one mass scale, $\Delta m_{32}^{2}$, and with maximal mixing between second and third families. One important prediction for these numbers is that the high-energy neutrino events that generate the through-going muon data are well described together with the lowenergy neutrino events, due to the energy dependence of the Hamiltonian that describes the neutrino evolution.

Assuming a non-vanishing NSNI acting together with mass and mixing, the solution to the atmospheric neutrino discrepancy can be spoiled if the NSNI parameters have too large values. This happens because the NSNI entries in the Hamiltonian that describes the neutrino evolution are energy independent [322]. Since a simultaneous explanation of low-energy and high-energy neutrino events requires a strong energy dependence in the $\nu_{\mu}, \nu_{\tau}$ conversion probability, inclusion of energy independent terms in the Hamiltonian tends to decrease the quality of the theoretical predictions fit to atmospheric neutrino data.

The NSNI can be parametrized as a relative strength of such interactions to the $\epsilon_{i j}^{f}=$ $\frac{G_{\nu_{i} \nu_{j}}^{f}}{G_{f}}$ where $f$ stands for the fermion involved in the new interaction: 


$$
\begin{aligned}
\epsilon & =\frac{G_{\nu_{\mu} \nu_{\mu}}^{d}-G_{\nu_{e} \nu_{e}}^{d}}{G_{f}}=\frac{G_{\nu_{\tau} \nu_{\tau}}^{d}-G_{\nu_{e} \nu_{e}}^{d}}{G_{f}}(\mathrm{~d}-\text { quarks }) \\
& =\frac{G_{\nu_{\mu} \nu_{\mu}}^{u}-G_{\nu_{e} \nu_{e}}^{u}}{G_{f}}=\frac{G_{\nu_{\tau} \nu_{\tau}}^{u}-G_{\nu_{e} \nu_{e}}^{u}}{G_{f}} \quad(\mathrm{u}-\text { quarks }) \\
& =\frac{G_{\nu_{\mu} \nu_{\mu}}^{e}-G_{\nu_{e} \nu_{e}}^{e}}{G_{f}}=\frac{G_{\nu_{\tau} \nu_{\tau}}^{e}-G_{\nu_{e} \nu_{e}}^{e}}{G_{f}} \quad \text { (electrons) }
\end{aligned}
$$

The solar neutrino data is able to stablish new limits in the flavor-diagonal NSNI (57). With the definitions written above, these limits stands for:

In [323] an analysis of atmospheric neutrinos and NSNI is performed, and found the following limits, at $3 \sigma$ :

$$
\begin{aligned}
\left|\epsilon_{\mu \tau}\right| & <0.03 \\
\left|\epsilon_{\mu \mu}^{\prime}-\epsilon_{\tau \tau}^{\prime}\right| & <0.05
\end{aligned}
$$

These bounds refer to NSNI with d-quarks, and were obtained assuming a two-flavor $\left(\nu_{\mu}\right.$ and $\left.\nu_{\tau}\right)$ system. A three family analysis significantly relaxes some of the bounds above [324], such that order one values for some of the $|\epsilon| \mathrm{s}$ are not currently ruled out. For many more details, see [324].

For u-quarks the bounds are expected to be of the same order, and for NSNI with electrons we expect bounds looser by a factor of $\sim 3$.

\subsubsection{KamLAND and solar neutrinos}

The excellent agreement between the LMA parameter region that provides a solution to the solar neutrino problem and the parameter region compatible with KamLAND dataprovides us with an opportunity to use these data sets to establish a limit on nonstandard neutrino interactions (NSNI).

The effect of NSNI is negligible in KamLAND due to the short distance traveled inside the earth, but due to the high density and long travel distances in the sun, the presence of a NSNI could displace the best fit point of solar neutrino analyses, and spoil the agreement between solar and KamLAND allowed regions.

The oscillation of solar neutrinos is driven by only one mass scale, $\Delta m_{21}^{2}$. The higher mass scale $\Delta m_{32}^{2}$, relevant for atmospheric neutrino oscillations, decouples, and the mixing between the first and third family is very small, and will be set to zero in what follows.

In this approach, after rotating out the third family from the evolution equation, we can write the $2 \times 2$ Hamiltonian that describes the neutrino evolution as

$$
\begin{aligned}
H_{M S W} & =\left[\begin{array}{cc}
+\sqrt{2} G_{F} N_{e}(r)-\frac{\Delta m^{2}}{4 E} \cos 2 \theta & \frac{\Delta m^{2}}{4 E^{2}} \sin 2 \theta \\
\frac{\Delta m^{2}}{4 E} \sin 2 \theta & \frac{\Delta m^{2}}{4 E} \cos 2 \theta
\end{array}\right] \\
+ & {\left[\begin{array}{cc}
0 & \sqrt{2} G_{F} \epsilon_{f} N_{f}(r) \\
\sqrt{2} G_{F} \epsilon_{f} N_{f}(r) & \sqrt{2} G_{F} \epsilon_{f}^{\prime} N_{f}(r)
\end{array}\right] }
\end{aligned}
$$


where $N_{f}(r)$ is an effective density felt by the neutrino, given by $N_{f}=N_{p}+2 N_{n}$ for d-quarks, $N_{f}=2 N_{p}+N_{n}$ for u-quarks and $N_{f}=N_{p}$ for electrons.

The NSNI parameters can be written as:

$$
\begin{aligned}
\epsilon_{f}^{\prime} & =\frac{\epsilon_{\tau \tau}+\epsilon_{\mu \mu}}{2}+\frac{\epsilon_{\tau \tau}-\epsilon_{\mu \mu}}{2} \cos 2 \theta_{23}-\epsilon_{\mu \tau} \sin 2 \theta_{23}-\epsilon_{e e} \\
\epsilon_{f} & =\epsilon_{e \mu} \cos \theta_{23}-\epsilon_{e \tau} \sin \theta_{23} .
\end{aligned}
$$

The atmospheric neutrino analyses have put strong bounds on $\epsilon_{\mu \tau}$ and $\epsilon_{\mu \mu}-\epsilon_{\tau \tau}$, and we expect a near to maximal mixing between second and third families $\left(\cos 2 \theta_{23} \simeq 0\right)$. By further assuming that $\epsilon_{f}=0$ (which would be the case if $\epsilon_{e \mu}$ and $\epsilon_{e \tau}$ were negligible) we can write $\epsilon_{f}^{\prime}$ as

$$
\epsilon_{f}^{\prime} \sim \epsilon_{\mu \mu}-\epsilon_{e e}=\epsilon_{\tau \tau}-\epsilon_{e e}
$$

With the present data set and the assumptions listed above, we are able to establish the following limits to the NSNI parameters, at $1 \sigma(2 \sigma)$ :

$$
\begin{array}{lll}
-0.20<\epsilon^{\prime}<0.12 \quad\left(\epsilon^{\prime}<0.30\right) & \text { d-quarks } \\
-0.18<\epsilon^{\prime}<0.10\left(\epsilon^{\prime}<0.30\right) & \text { u-quarks } \\
-0.55<\epsilon^{\prime}<0.25\left(\epsilon^{\prime}<0.86\right) & \text { electrons } .
\end{array}
$$

The limits obtained at $2 \sigma$ reflect the weak bounds on $\Delta m^{2}$ obtained by KamLAND. At present there are a number of possible "islands" in the parameter region compatible with KamLAND data, so the displacement in $\Delta m^{2}$ could make the best fit point of a combined analysis jump between consecutive islands. The increase of statistics at KamLAND will determine in which of these islands the true values of neutrino parameters lie, avoiding this kind of jump and improving the limits on the NSNI parameters.

Simulating 1 kton-year of data at KamLAND, the allowed range of $\Delta m^{2}$ would be reduced, and in the case of an agreement between the neutrino parameters coming from KamLAND and solar neutrino analysis (that would indicate a vanishing NSNI) new limits could be obtained. Although the $1 \sigma$ regions do not change significantly, at $2 \sigma$ we have:

$$
\begin{array}{r}
-0.42<\epsilon^{\prime}<0.24 \quad \text { (d-quarks) } \\
-0.40<\epsilon^{\prime}<0.18 \quad \text { (u-quarks) } \\
\epsilon^{\prime}<0.40 \quad \text { (electrons). }
\end{array}
$$

Further increase of KamLAND statistics will not improve these bounds, which are now determined by the uncertainty in $\Delta m^{2}$ in the solar neutrino analysis.

Details of the analysis presented here can be found in [325]. Similar analyses were also done in [326]. It should be pointed out that more severe constraints on (or a positive hint

of) NSNI can be obtained in next-generation solar neutrino experiments, especially those sensitive to neutrino energies below $6 \mathrm{MeV}$ [326].

\subsubsection{Bounds from non-oscillating phenomena}

Apart from phenomena that involve neutrino oscillations, bounds on NSNI can also come from the effects of such non-standard interactions on the charged leptons [327,328]. We 
should only be careful in translating such bounds to the neutrino sector, since usually this translation can only be possible with a few assumptions on the model that generates the non-standard interactions. Some bounds can also be found using neutrino scattering experiments $[329,330]$. We quote here some of the numbers obtained by these analyses. Details of the calculations can be found in the references. The following tables should be read as limits on $\epsilon_{i, j}$, where $i$ and $j$ stand for $e, \mu$ and $\tau$, and are the lines and columns of the tables.

The values quoted here depend on details of the models that generate the NSNI, such as $S U(2)_{L}$ breaking effects, absence of fine-tuning cancellations and the scale of new physics. Also, since neutrino oscillations are sensitive only to the vector coupling constant of the NSNI, correlations between the limits in $\epsilon_{L}$ and $\epsilon_{R}$ should be taken into account in order to compare the numbers presented here with the ones coming from neutrino oscillation experiments.

\section{Electrons:}

\begin{tabular}{c|c|c|c} 
& $e$ & $\mu$ & $\tau$ \\
\hline$e$ & $-0.07<\epsilon_{L}<0.1$, & $\epsilon<10^{-6}[328]$ & $\epsilon<4.2 \times 10^{-3}[328]$ \\
& $-1<\epsilon_{R}<0.5[330]$ & $\epsilon_{L, R}<5 \times 10^{-4}[330]$ & $\left|\epsilon_{L}\right|<0.4,\left|\epsilon_{R}\right|<0.7[330]$ \\
\hline$\mu$ & & $\left|\epsilon_{L, R}\right|<0.03[330]$ & $\epsilon<3.1 \times 10^{-3}[327]$ \\
\hline$\tau$ & & $\epsilon_{L, R}<0.1[330]$ \\
\hline$\tau$ & & {$[-0.05 ; 0.05]_{L, R}[330]$}
\end{tabular}

\section{d-quarks:}

\begin{tabular}{c|c|c|c} 
& $e$ & $\mu$ & $\tau$ \\
\hline$e$ & $\left|\epsilon_{L}\right|<0.3$, & $\epsilon<10^{-5}[328]$ & $\epsilon<10^{-2}[328]$ \\
& $-0.6<\epsilon_{R}<0.5[330]$ & $\epsilon_{L, R}<7.7 \times 10^{-4}[330]$ & $\epsilon_{L, R}<0.5[330]$ \\
\hline$\mu$ & & $\epsilon<0.1[328]$ & $\epsilon<1.2 \times 10^{-2}[327]$ \\
& & $\left|\epsilon_{L}\right|<0.003,-0.008<\epsilon_{R}<0.015[330]$ & $\epsilon_{L, R}<0.05[330]$ \\
\hline$\tau$ & & & $\left|\epsilon_{L}\right|<1.1$, \\
& & & $\left|\epsilon_{R}\right|<6[330]$
\end{tabular}

\section{u-quarks:}

\begin{tabular}{c|c|c|c} 
& $e$ & $\mu$ & $\tau$ \\
\hline$e$ & $-1<\epsilon_{L}<0.3$, & $\epsilon<10^{-5}[328]$ & $\epsilon<10^{-2}[328]$ \\
& $-0.4<\epsilon<0.7[330]$ & $\epsilon_{L, R}<7.7 \times 10^{-4}[330]$ & $\epsilon_{L, R}<0.5[330]$ \\
\hline$\mu$ & & $\epsilon<0.1[328]$ & $\epsilon<1.2 \times 10^{-2}[327]$ \\
& & $\left|\epsilon_{L}\right|<0.003,-0.008<\epsilon_{R}<0.003[330]$ & $\epsilon_{L, R}<0.05[330]$ \\
\hline$\tau$ & & $\left|\epsilon_{L}\right|<1.4,\left|\epsilon_{R}\right|<3[330]$
\end{tabular}

Concluding, using the neutrino oscillation data we are able to find limits to NSNI parameters, without assuming any detail about the nature of new physics behind these interactions. More work is needed to improve the situation. 


\section{Beyond the three neutrino picture}

\subsection{The search for other light neutrinos}

A neutrino that does not participate in Standard Model interactions (i.e., is sterile) might seem of little interest, but this concept includes reasonable theoretical constructs such as right-handed neutrinos themselves. We note in passing that in one-family Technicolor theories there are also technineutrinos that would couple with the usual strength to the $W$ and $Z$ but, because of their Technicolor interactions, are confined and gain large dynamical masses of order several hundred GeV; they are therefore not relevant for usual low-energy neutrino oscillation experiments. The hypothesis of 'sterility' concerns the weak forces; gravity is expected to be felt anyway, and we cannot exclude that the 'sterile' neutrino participates in new forces, perhaps, mostly coupled to quarks; or carried by new heavy mediators; or that sterile neutrinos have preferential couplings with new particles - say, with Majorons. Even putting aside these possibilities, we can probe sterile neutrinos by the search for observable effects due to their mixing with the ordinary neutrinos. In this section, we will further restrict our attention on 'light' sterile neutrinos (say, below $10 \mathrm{eV}$ ) and discuss the impact on oscillations. We make extensive reference to Ref. [331], an updated overview on the phenomenology of one extra sterile neutrinos.

\subsubsection{Issues of theoretical justification}

Many extensions of the Standard Model incorporate particles behaving as sterile neutrinos. The main question is: Why are these light and do they have the couplings needed to mix with ordinary neutrinos? A recent discussion is in Ref. [95]. Models with mirror matter [332,333] contain mirror neutrinos [334,335] and offer a straightforward answer: ordinary and mirror neutrinos are light for the same reason. It is easy to arrange a 'communication' term between ordinary and mirror worlds, e.g., due to the operator $\sim \nu \phi \nu^{\prime} \phi^{\prime} / M_{\mathrm{Pl}}$. This leads to long-wavelength oscillations into sterile neutrinos. (see Fig. [17. from [339]). There are many other possibilities. For example, higher-dimensional operators in the superpotential in models involving a scalar field with an intermediate scale expectation value can naturally lead to small Dirac and Majorana masses of the same magnitude, and therefore to light ordinary and sterile neutrinos which can mix [95]. Already with mirror matter, the $\operatorname{VEV}\left\langle\phi^{\prime}\right\rangle$ could be different from $\langle\phi\rangle=174 \mathrm{GeV}$, and this has important carryings for the phenomenology [334,335]. Alternatively, one could guess on dimensional ground the value $\mathrm{TeV}^{2} / M_{\mathrm{Pl}}$ as the mass (or mixing) of sterile neutrinos, and relate the $\mathrm{TeV}$-value, e.g., to supersymmetry breaking [336] or high GUT theories such as $E_{6}$ [337]. In theories with dynamical electroweak symmetry breaking, the mechanism that has been found for light neutrinos predicts also that there are sterile neutrinos with masses of order 100's of MeV to GeV [92, 93,441,442].

Right-handed neutrino masses of about 1 or $100 \mathrm{TeV}$ lead to exceedingly large masses that pose a question for extra dimensions if "large" scales should be absolutely avoided and if the Dirac Yukawa couplings are not small on their own. A popular way out is to postulate that the masses are Dirac in character. As a benefit, one explains why neutrino masses are small when right-handed neutrinos propagate in the bulk [338]. But even if neutrinos turn out to be Dirac particles we would not have an evidence for these 
theories, since (1) Dirac neutrino masses are possible in conventional 4-dimensional theories; (2) even in theories with extra dimensions, one can assume that the usual dim.-5 term is the dominant source of neutrino masses. We just need to fix the scale of mass to the desired value of $10^{13-15} \mathrm{GeV}$, and neutrinos receive (presumably large) Majorana masses [339]. To summarize, small neutrino masses are possible, and even more interestingly an infinite number of sterile neutrinos, but neutrino masses seem not to be a clean signal of low scale gravity theories. Oscillations have special character and there are interesting phenomenological constraints [340].

We believe that there should be increased attention paid not only toward phenomenology, but also toward theories of (light- or heavy-mass) sterile neutrinos. The fact that we do not understand usual fermion masses should be not taken as an excuse to avoid confrontation with theory in our view. With these considerations in mind, we will give some emphasis to mirror neutrinos, and at the same time point out the interest of understanding the unknown $\mathcal{O}(1)$ coefficients in these theories, that have an important impact on the phenomenological implications.

\subsubsection{Phenomenological manifestations}

In the following discussion, we will be concerned mostly with oscillations. However, the implications can be also elsewhere. To see that, it is sufficient to recall that when we add 3 sterile neutrinos we can form Dirac masses, which means that there is no contribution to the neutrinoless double beta decay process.

Terrestrial oscillation experiments Broadly speaking, there are two types of terrestrial experiments. The first one includes several disappearance experiments and LSND; the second one includes atmospheric neutrinos and long-baseline experiments. The first type is sensitive mostly to the mixing of $\nu_{e}$ and a sterile state, the other one also to $\nu_{\mu}$ or $\nu_{\tau}$. Both types of experiments probe only relatively large mixing angles, $\theta_{s} \sim 0.1$. Sterile neutrinos within the sensitivity regions are disfavored if standard cosmology (mostly $\mathrm{BBN}$ ) applies; further important tests will be done by CMB+LSS or BBN data. None of these experiments alone requires the existence of sterile neutrinos. A case for sterile neutrinos can be made interpreting in terms of oscillations LSND together with solar and atmospheric data [341]. The hypothesis that LSND signal is due to a relatively heavy and mostly sterile neutrino should be regarded as conservative [342], even though it leads to some problems with disappearance in terrestrial experiments, and interesting predictions for cosmology (BBN and CMB+LSS spectra). In view of this situation, the test of the LSND result is of essential importance. At the same time, we should not forget that sterile neutrinos could manifest themselves in other manners.

Solar and KamLAND neutrinos The solar and KamLAND data can be explained well without sterile neutrinos. Even more, the 'LMA' solution received significant confirmations: the sub-MeV energy regions have been probed by Gallium experiments and the super-MeV ones by SNO and Kamiokande, and LMA is in agreement with KamLAND. Thus we are led to consider minor admixtures of sterile neutrinos, presumably not more than $20 \%$. In many interesting cases sterile neutrinos are invisible at KamLAND but 

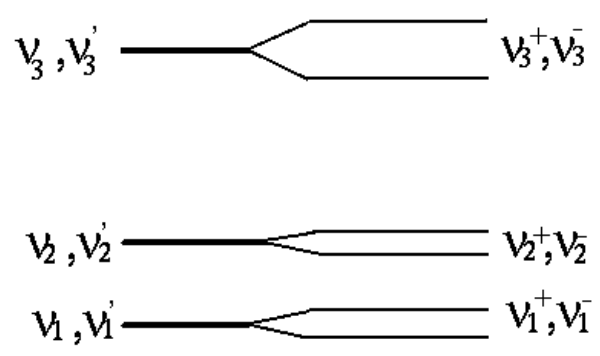

Figure 17: The double degeneracy between mass eigenstates of ordinary and mirror world $\left(\nu_{i}\right.$ and $\left.\nu_{i}^{\prime}\right)$ is lifted when the small mixing terms are included in the $6 \times 6$ mass matrix. The new mass eigenstates $\left(\nu_{i}^{+}\right.$and $\left.\nu_{i}^{-}\right)$are in good approximation maximal superpositions of $\nu_{i}$ and $\nu_{i}^{\prime}$.

affect the survival probability of solar neutrinos. Quite generally, to test the hypothesis of oscillations into sterile states it would be important to improve on (or measure precisely) the fluxes from Beryllium and pp-neutrinos. A few selected cases are shown in Fig. 18 from [331].

Neutrinoless double beta decay Massive Majorana sterile neutrinos, mixed with the active ones, would participate in mediating neutrinoless double beta decay. In the case of one light sterile neutrino, $\langle m\rangle_{e f f}$ is given by the sum of the contributions of four massive neutrinos [343]:

$$
\langle m\rangle_{e f f}=\sum_{i=1,4} m_{i} U_{e i}^{2},
$$

where $m_{i}$ denotes the mass of the massive eigenstates and $U_{e i}$ indicates their mixing with the electron neutrino.

Supernovae Supernovae are an ideal place to search for manifestations of sterile neutrinos, either through long wavelength vacuum oscillations of a galactic supernova (whose distance is in the kpc scale-SN1987A was at $52 \mathrm{kpc}$ ) or through MSW effects (since nuclear densities are reached during the collapse); the main trouble is that we are still not able to understand supernova explosions theoretically (the data from SN1987A do not contradict the simplest current picture for neutrino emission, but they have puzzling features that suggest caution). Vacuum oscillations induced by mirror neutrinos (as in Fig. 17) can led to a disappearance of half of the emitted flux [339]. MSW oscillations into sterile neutrinos can produce even more dramatic suppressions; in certain regions of the parameter space this can reach $80 \%$ [331]. The last type of oscillations are due to the fact that the SN core is deleptonized, and require that the electron neutrino mixes with the sterile one. The trouble to verify these predictions is the accuracy of the expectations on the emitted neutrino energy, that amounts roughly to a factor of 2 ; thus it seems that, with present knowledge of supernovae, and using the data from SN1987A, we can only safely exclude 

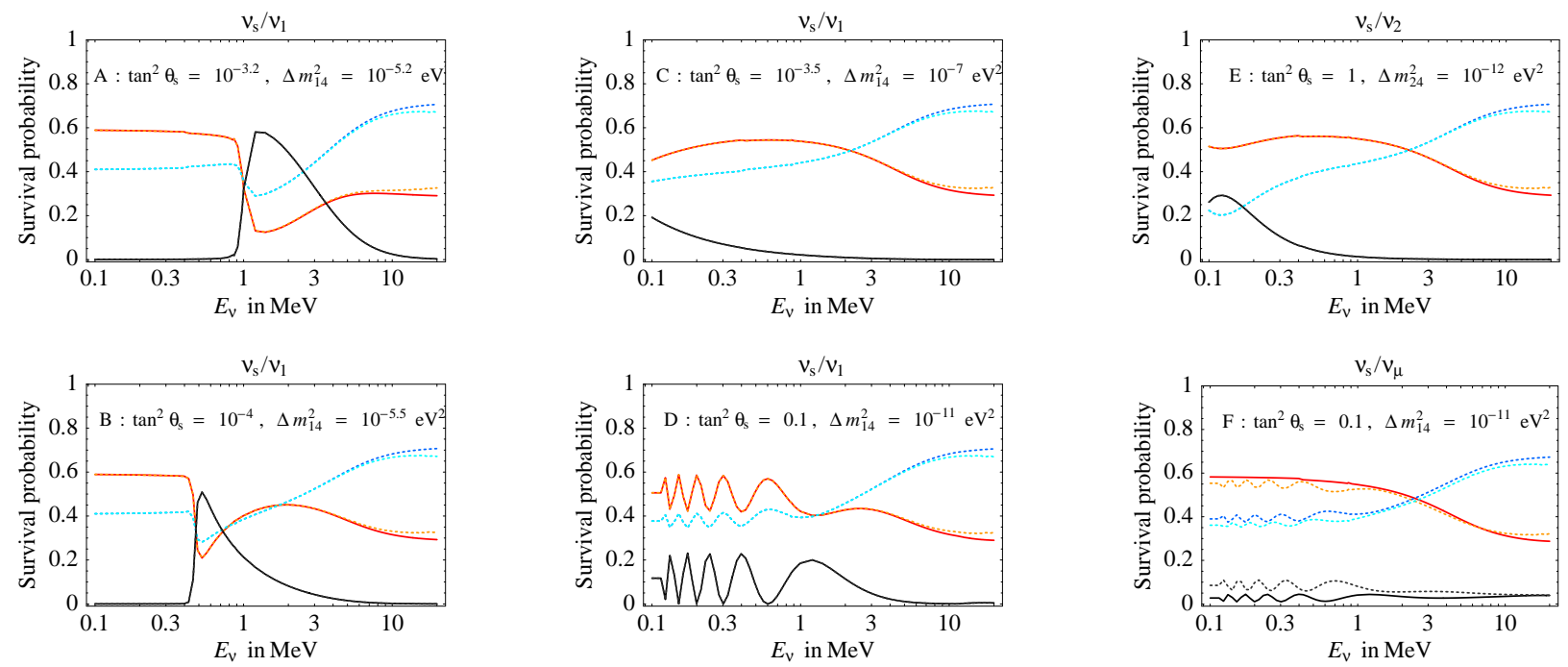

Figure 18: Examples of oscillation into sterile neutrinos allowed from present solar neutrino data. In the plots are shown the 3 probabilities of oscillations $P\left(\nu_{e} \rightarrow \nu_{e}\right)$ (decreasing red curve), $P\left(\nu_{e} \rightarrow \nu_{\mu, \tau}\right)$ (increasing blue curve) and $P\left(\nu_{e} \rightarrow \nu_{\mathrm{s}}\right)$ (lower black curve), as a function of the neutrino energy. The continuous (dotted) curve are the values during day (night).

the occurrence of dramatic MSW effects. We remark that with a quantitative theory of core collapse supernovae, this test of sterile neutrinos would become very powerful. There are other interesting effects possibly related to heavy sterile neutrinos, such as r-process nucleosynthesis, re-heating of the shock, rocketing of pulsars, not discussed here.

Big-bang nucleosynthesis and other cosmological probes An often stressed doubt concerning cosmology is about neglected or unknown effects. This said, we must recall that there has been impressive progress in the last years. The impact on neutrinos can be summarized as follows: (1) The number of neutrinos at the photon decoupling is bounded to be $3 \pm 2$. (2) The contribution to the energy density of the universe $\Omega_{\nu} h^{2}$ is below or at about $1 \%$, or, equivalently, the sum of neutrino masses is below or at about $1 \mathrm{eV}$. (It should be recalled that there is an interesting, not universally accepted claim that the cosmology gives not a bound but a value for neutrino masses [344]. This testifies the high sensitivity reached by these methods and points to the interest in the value of the bias parameter $\sigma_{8}$ ). (3) The effective number of neutrinos at nucleosynthesis time is $3 \pm 2$, when extracted from deuterium abundance, or $2.4 \pm 0.7$, when extracted from helium abundance. These numbers already imply strong bounds on sterile neutrinos, but do not rule out the sterile hypothesis for interesting regions of the parameters. One example is given by mirror neutrinos, when the new mass splittings are small enough. Another one is given by a new sterile state, that has only small mixing with the ordinary neutrinos. A second possibility is to have post-BBN phase transition involving the vacuum expectation value 
of a light scalar field which mixes the active and sterile neutrinos so that at the time of $\mathrm{BBN}$, the active and sterile neutrinos are unmixed. There are however strong constraints on the nature and interaction of the scalar field [94,345] and an interesting feature of these models is that they leave an imprint on the cosmic microwave background that can be tested in future experiments such as the Planck satellite mission. Yet another possibility is a large electron neutrino asymmetry in the early universe, which can compensate the effects of a number of extra neutrino types [346].

It has also been pointed out that the production of sterile neutrinos in the Early Universe is strongly suppressed in cosmological scenarios for which the reheating temperature is as low as few $\mathrm{MeV}$ [347]. In this case the bounds on the sterile neutrino parameters which can be derived from BBN and from the contribution of $\Omega_{\nu} h^{2}$ to the Dark Matter are much weaker than in the standard case.

Ultra-high energy neutrinos Although there is a great interest in the search for ultrahigh energy neutrinos, the number of reasonable (or even, less reasonable) mechanisms that have been discussed to produce them is not large. The reason is that neutrinos are produced along with electromagnetic radiation, that can be observed in a variety of ways, even when this is reprocessed. Following this line of thought, the astrophysical mechanism that can be conceived to overcome such a structure is the concept of a 'hidden source'. Another escape way from this constraint involves sterile neutrinos. Indeed, if there are ultra-high energy mirror neutrinos, they inevitably oscillate into neutrinos from our world on cosmic scales [348]. This scenario can provide intense fluxes of ultra-high energy neutrinos, subject only to the observable electromagnetic radiation from their interaction with the relic neutrino sea.

\subsubsection{Summary of what we can learn on light sterile neutrinos}

In the view of many, there is something embarrassing in the hypothesis of (light) sterile neutrinos, and some of us believe that this is 'a solution searching for a problem'. However, history tells that the most prominent characteristic of neutrinos is that they are amazing! Said more seriously, we should certainly aim to measure neutrino properties, but we should not forget that we could make discoveries. And, when we think to new experiments, we should evaluate their potential for the investigation of sterile neutrinos.

In this section, we recalled that light sterile neutrinos can play a role not only for LSND but also in terrestrial oscillations experiment, solar neutrinos, supernovae, astrophysics and cosmology. There are links between the various observables, but it is not impossible to conceive that sterile neutrinos have an important role only in astrophysics or cosmology (e.g., in core collapse supernovae or big-bang nucleosynthesis). More measurements and theoretical progresses will lead to important tests of the idea that the neutrinos that we know are not the full story.

\subsection{What can we learn about four-neutrino mass matrices}

The solar, atmospheric and LSND data require three different (mass) ${ }^{2}$ splittings. These cannot be accommodated by three neutrino flavors, which provide only two independent 
$\Delta m^{2}$. Additional degrees of freedom are necessary in order to understand all this data put together. The easiest option is to add a sterile neutrino and interpret the data in terms of oscillations of four neutrino flavors.

The MiniBooNE experiment at Fermilab is crucial for confirming or refuting the LSND evidence for neutrino oscillations. If the LSND result is confirmed, a very exciting epoch in neutrino physics is just about to begin, as the number of questions that need to be answered becomes even larger than in the standard three-flavor case.

A general 4-neutrino Majorana mass matrix is described by 4 masses, 6 mixing angles and $6 \mathrm{CP}$ violating phases, 3 of which would affect oscillations.

In this case there are 6 possible mass spectra, as shown in Fig. 19] These can be divided in two categories: " $3+1$ " and " $2+2$ ". The " $3+1$ " mass patterns are comprised of one sterile neutrino separated by $\Delta m_{\mathrm{LSND}}^{2}$ from the other three. The group of three is the usual group of "active" neutrinos, one pair separated by $\Delta m_{\odot}^{2}$ and the third separated from these by $\Delta m_{\mathrm{A}}^{2}$. The " $2+2$ " patterns are comprised of two pairs of neutrinos, one separated by $\Delta m_{\odot}^{2}$ and the other by $\Delta m_{\mathrm{A}}^{2}$. The two pairs are separated by $\Delta m_{\mathrm{LSND}}^{2}$.

Both categories are already very strongly constrained by experiment $[349,350]$.

In the " $3+1$ " scenario the 3 states relevant for solar and atmospheric oscillations are mostly active and the forth state is almost entirely sterile. This pattern has the usual three active flavor scenario as a limiting case, so it agrees very well with all solar and atmospheric data. It is however harder to accommodate short baseline neutrinos oscillation experiments. This is related to the irony of the fact that LSND is an active flavor appearance experiment, showing $\bar{\nu}_{\mu} \rightarrow \bar{\nu}_{e}$ oscillations, but its solution has to involve an almost entirely sterile neutrino, while the other experiments remain unaffected by the presence of the sterile state. The bounds on $\sin ^{2} 2 \theta_{\text {LSND }}$ coming from KARMEN, CDHS, $\mathrm{CHOOZ}$ and atmospheric data are rather strong, almost conflicting with the value required to explain the LSND signal. The fit to all data is not very good, but the " $3+1$ " scenario is not completely excluded at this point.

In the ' $2+2$ " scenario both solar and atmospheric neutrino oscillations involve some fraction of conversion into a sterile state. This fractions are now very strongly constrained by the atmospheric, solar and reactor data, making the fit to the data to be rather poor in the " $2+2$ " case. The global analysis are usually performed by considering three mixing angles and neglecting the other three, which are known to be small. Including these additional small angles might improve the quality of the fits, but the " $2+2$ " scenario is strongly disfavored.

The presence of the fourth, sterile neutrino also has implications in cosmology and cosmological observations impose further constraints on the allowed parameter space, as discussed in the previous section.

The first question regarding four neutrino mass matrices, namely if they are indeed necessary to interpret the experimentally observed neutrino oscillation data will soon be answered by the MiniBooNE experiment. Assuming the answer is positive, a whole new set of questions arises. Just as in the three flavor case, one would like better measurements of all $\Delta m^{2}$ 's and mixing angles. Given the much larger number of phases involved in the 4 neutrino case, the possibilities for observing $\mathrm{CP}$ violation in the neutrino sector become very rich and maybe more easily accessible [351]. If the LSND signal is confirmed, than there must be a state with mass higher than $\sqrt{\Delta m_{\mathrm{LSND}}^{2}}$. This would be in the range of 
sensitivity of future tritium $\beta$ decay experiments like KATRIN, raising the possibility of determining the absolute scale of neutrino mass. For Majorana neutrinos, neutrinoless double beta decay might also be accessible $[343,352]$. By combining data from all types of experiments, the specific mass pattern could also be determined.
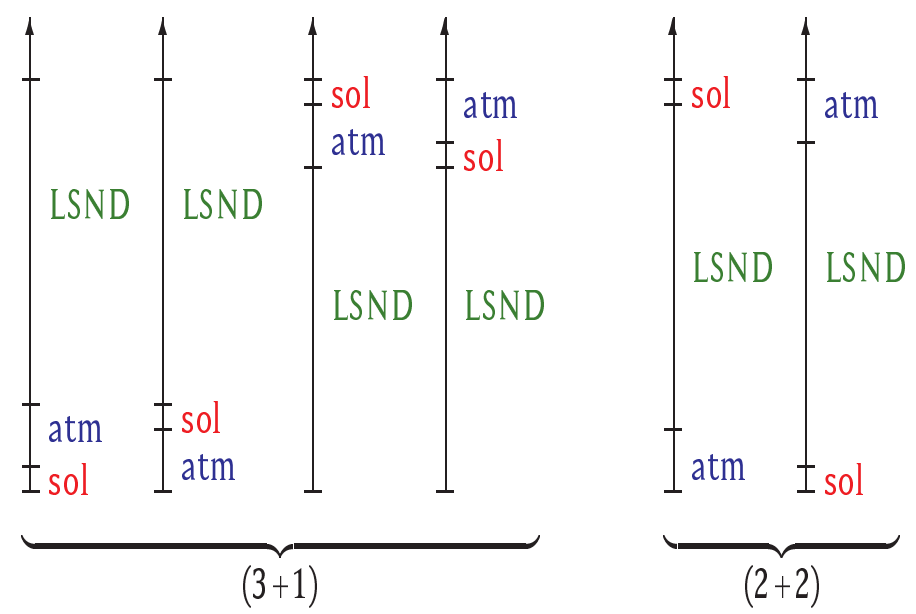

Figure 19: Four neutrino mass spectra.

\subsection{Heavy sterile neutrinos}

The general motivation for considering sterile neutrinos has already been discussed. Many extensions of the Standard Model imply the existence of more than one sterile neutrino with couplings to the active ones. The right-handed neutrinos participating in the seesaw mechanism, mirror neutrinos, the neutrinos in extra-dimensional models, and the righthanded technisinglet neutrinos in the ETC model discussed in other sections are some examples of such sterile neutrinos.

"Light" (below $\sim 10 \mathrm{eV}$ ) sterile neutrinos have been discussed in Sec. [7.1. Here we concentrate on "heavy" ones, by which we mean sterile neutrinos with masses above $10 \mathrm{eV}$, but below $\sim 1 \mathrm{GeV}$. As noted, the mechanism constructed in Refs. [92, 93, 441] for light neutrinos in Technicolor theories leads to (two) heavy neutrino mass eigenstates in this range. We do not discuss here "very heavy" neutrinos (e.g. GUT scale), whose properties have been talked about above. Once sterile neutrinos are introduced, there is no definitive prediction for either the number or the masses of these light neutral fermions. Answering the question of the total number of neutrinos (active and sterile) and their masses is fundamental, as it would lead to much progress in understanding physics beyond the Standard Model. It is thus very important to address these issues from the experimental/observational point of view.

Heavy sterile neutrinos with couplings to the active ones have profound implications in cosmology and astrophysics. They can also be constrained by several types of laboratory experiments. We discuss here the present status and future prospects for determining the properties of these heavy sterile neutrinos. 


\subsubsection{Laboratory Experiments}

The existence and mixing of heavy sterile neutrinos has many effects on particle and nuclear decays. These include contributions to $\mu^{+} \rightarrow e^{+} \gamma, \mu^{+} \rightarrow e^{+} e^{+} e^{-}, K_{L} \rightarrow \mu^{ \pm} e^{\mp}$, and $K^{+} \rightarrow \pi^{+} \mu^{ \pm} e^{\mp}$, among others. However, given limits on mixing, these contributions are expected to be quite small (see further below on $\mu^{+} \rightarrow e^{+} \gamma$ ).

The two-body leptonic decays of charged pions and kaons, and also measurement of the differential decay distribution in $\mu$ decay, can be used to search for, and set bounds on, the emission of massive neutrinos via lepton mixing [353,354]. The experimental signature for the emission of a massive neutrino via lepton mixing in $\pi_{\ell 2}^{+}$or $K_{\ell 2}^{+}$decay would be the appearance of an additional peak in the momentum spectrum of the outgoing charged lepton $\ell^{+}=\mu^{+}$or $e^{+}$. The position of the extra peak is determined by the mass of the heavy neutrino and the size of the extra peak is proportional to the mixing $\left|U_{\ell h}\right|^{2}$, where $\ell=e$ or $\mu$, between the extra state and the neutrino $\nu_{\ell}$. Initial bounds from retroactive data analyses were given in [353], and dedicated searches were carried out in $\pi_{\mu 2}^{+}$[355], $K_{\mu 2}^{+}$[356], $\pi_{e 2}^{+}$[357], and $K_{e 2}^{+}$[358] decays. Because of renewed interest [359], some recent searches in $\pi_{\mu 2}^{+}$are reported in [360]. Resultant upper bounds on $\left|U_{\ell h}\right|^{2}$ range down to $10^{-5}$ $-10^{-7}$ in the mass range from several $\mathrm{MeV}$ to $\sim 300 \mathrm{MeV}$. Admixed massive neutrinos also affect the observed ratio of branching ratios $B R\left(\pi_{e 2}^{+}\right) / B R\left(\pi_{\mu 2}^{+}\right)$and this has been used to set limits (e.g., [361]).

The nuclear beta decay spectrum is also affected by the presence of heavy neutrinos mixed with $\nu_{e}$. The spectrum would have a "kink" at the endpoint energy $E_{\text {max }}\left(m_{h}\right)$ $[353,362-364]$. The position of the kink determines the mass of the heavy state, $m_{h}$, and the change in the slope of the spectrum determines the mixing $\left|U_{e h}\right|^{2}$. Many experiments searching for such kinks in the Kurie plots of nuclear beta decays were carried out in the 1980's and beginning of the 1990's; although a few claimed positive results, these were refuted [3]. Resultant upper limits were of the order $\left|U_{e h}\right|^{2} \approx 10^{-3}$ for masses between 10 $\mathrm{keV}$ and $\sim 1 \mathrm{MeV}$.

The nuclear transition involving electron capture, $e^{-}+(Z, A) \rightarrow(Z-1, A)+\nu_{e}[365]$ and muon capture, $\mu^{-}+(Z, A) \rightarrow(Z-1, A)+\nu_{\mu}$ [366] can also be used to search for, and put limits on, massive neutrino emission via mixing.

Assuming that the additional sterile neutrinos are Majorana, there are several $|\Delta L|=$ 2 transitions and meson and hyperon decays (processes analogous to neutrinoless nuclear double beta decay) that can occur. One of these is the nuclear transition $\mu^{-}+(A, Z)->$ $\mu^{+}+(A, Z-2)$ [367]. Meson decays include $K^{+} \rightarrow \pi^{-} \mu^{+} \mu^{+}$. A first upper limit on the branching ratio for this decay was set in [368], and this has been greatly improved by a dedicated search in a recent BNL experiment [369] (see also [370-373]). Analogous $|\Delta L|=2$ decays of heavy-quark mesons are also of interest. Early searches include one by the Mark II detector at PEP for the decays $D^{+} \rightarrow K^{-} \mu^{+} \mu^{+}$and $D^{+} \rightarrow \pi^{-} \mu^{+} \mu^{+}$[374] and one by the CLEO experiment at CESR for $B^{+} \rightarrow K^{-} \mu^{+} \mu^{+}$[375]; current limits are given in Ref. [3]. One can also consider $|\Delta L|=2$ hyperon decays such as $\Xi^{-} \rightarrow p \mu^{-} \mu^{-}$ and $\Sigma^{-} \rightarrow p \mu^{-} \mu^{-}$. A first upper limit, on $B R\left(\Xi^{-} \rightarrow p \mu^{-} \mu^{-}\right)$, was set in Ref. [376]; a recent dedicated search reporting a much improved limit is by the HyperCP experiment at Fermilab [377].

Mixing between heavy and light neutrinos also leads to neutrino decay [378-380]. 
A number of searches for neutrino decays in accelerator experiments have been carried out [381]. Depending on the assumed mass of the neutrino mass eigenstate, various decays are possible, including $\nu_{h} \rightarrow \nu^{\prime} e^{+} e^{-}, \nu_{h} \rightarrow \nu^{\prime} \mu^{ \pm} e^{\mp}, \nu_{h} \rightarrow \nu^{\prime} \mu^{+} \mu^{-}$. Bounds on various combinations of mixing angle factors from these experiments are reported in [381]; published limits range down to $\left|U_{\ell h}\right|^{2}<10^{-9}, \ell=e, \mu$, for heavy neutrino masses of several hundred $\mathrm{MeV}[3,381]$. In addition to weak charged current contributions to these neutrino decays, there are also contributions from the weak neutral current, since, in the presence of sterile neutrinos, the weak neutral current is not, in general, diagonal in mass eigenstates $[40,41,382]$. Present and future experiments as MiniBooNE and MINOS might have the possibility to improve on some of the present bounds on heavy neutrino decays [382]. Searches for heavy neutrino production and decay have also been carried out at $e^{+} e^{-}$colliders; see [3] for limits. For masses between a few $\mathrm{GeV}$ and $m_{Z}$ the best bounds come from measurements at the $\mathrm{Z}$ pole, where the $\mathrm{Z}$ boson could decay into a standard neutrino and a sterile one, that would further decay [3].

\subsubsection{Astrophysics and cosmology}

The existence of sterile neutrinos with even very small mixing to the active ones can have dramatic consequences in astrophysics and cosmology. These are discussed by a different working group [9]. Because astrophysical and cosmological observations provide the strongest constraints and prospects for future answers regarding heavy sterile neutrinos, we do, however, include here an overview of this subject.

\section{Cosmology}

Massive sterile neutrinos could be produced in the early universe and can provide some or even all the required dark matter. Heavy sterile neutrinos can be produced by scattering-induced conversion of active neutrinos [383]. These neutrinos are produced non-resonantly and they can be a warm dark matter candidate. A different mechanism of production of heavy sterile neutrinos appears if there is a non-vanishing initial lepton number in the Universe [384]. In this case sterile neutrinos can be produced resonantly and the energy spectrum is in this case highly non-thermal. The sterile neutrino can act then as a warm, cool or even cold dark matter [385].

Cosmological observations impose strong constraints on massive sterile neutrinos [385, 386]. The radiative decay of such neutrinos to a light neutrino and a photon would affect the diffuse extragalactic background radiation, by producing a large number of photons of energy of the order $m_{H}$. The DEBRA experiment is now constraining the parameter space of sterile neutrinos based on this. The Chandra X-ray observatory has a great potential of resolving a considerable fraction of the observed X-ray background and consequently imposing much stronger constraints or potentially detecting X-ray fluxes from dark matter sterile neutrinos in the gravitational potential wells of clusters of galaxies.

Heavy sterile neutrino decay prior to cosmic microwave background (CMB) decoupling increases the energy density in relativistic particles, leading to further constraints on the allowed parameter space.

Big bang nucleosynthesis (BBN) is one of the big successes of the Standard Model of cosmology, successfully predicting the primordial abundance of light elements. The energy density in the sterile neutrino sea prior to BBN must not be too high in order not 
to spoil the successful predictions of BBN. Photoproduction of deuterium and ${ }^{6} \mathrm{Li}$ from decay of sterile neutrinos after BBN also imposes additional constraints on the sterile neutrino parameter space.

Large scale structure observations are also essential, as they can constrain the nature of the dark matter (hot, warm or cold), consequently setting the scale for the mass of the sterile state.

Cosmological constraints are illustrated in Fig. 20 (from [385]).

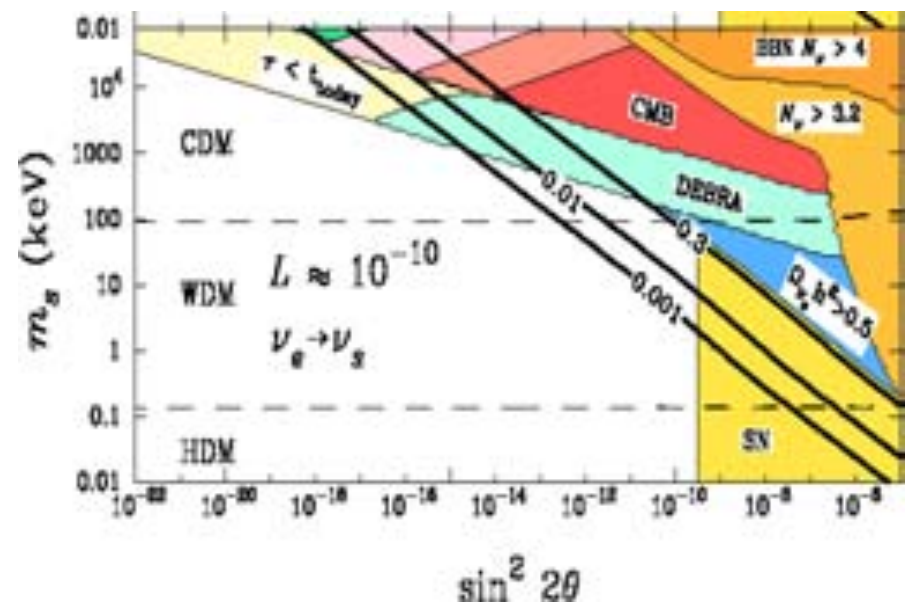

Figure 20: Cosmological/astrophysical constraints on heavy sterile neutrinos.

\section{Supernovae}

Neutrinos play a dominant role in core collapse supernovae. Even small admixtures of heavy sterile neutrinos can have profound implications for supernova physics. Too much active neutrino conversion into sterile states can lead to too much energy loss to sterile neutrinos, contradicting observations from supernova SN1987a [385,386]. The energy emitted in sterile neutrinos depends on the mixing angle between the sterile and active states in matter. For a long time it has been thought that most of the emission occurs in the resonant region, where the effective mixing angle becomes $\pi / 4$. This is not necessarily true because of a non-linear effect that limits the resonant emission [385]. The effective matter potential for the $\nu_{e} \leftrightarrow \nu_{s}$ is given by

$$
V=G_{F} \rho / \sqrt{2} / m_{N}\left(3 Y_{e}-1+4 Y_{\nu_{e}}+2 Y_{\nu_{\mu}}+2 Y_{\nu_{\tau}}\right)
$$

where $Y_{i}=\left(n_{i}-n_{\bar{i}}\right) / n_{B}$. For antineutrinos the matter potential changes sign. Due to the presence of the $Y_{\nu}$ terms, coming from neutral current neutrino-neutrino scattering, this effective potential has zero as a fixed point. Once a resonance is reached for, let's say, neutrinos, $\nu_{e}$ 's start converting with maximal effective mixing angle into $\nu_{s}$. This decreases the $Y_{\nu}$ term, driving the parameters off resonance and thus limiting the emission. The matter potential is effectively driven to zero on a time scale of less than a second and the emission continues with vacuum mixing angle. The parameter space where the sterile neutrino emission from supernovae could be relevant is also marked on Fig. 20.

It is interesting to note that a sterile neutrino in the few $\mathrm{keV}$ region could also account for pulsar kicks [387]. In the presence of a strong magnetic field, neutrinos are emitted 
asymmetrically in a supernova core. This asymmetry is washed out for active neutrinos which are trapped. If some conversion to sterile neutrinos occurs, these can escape the core of the star preserving the initial asymmetry. Only a few percent asymmetry is sufficient to account for the initial kick of the star that would explain the unusually high velocities of pulsars.

To summarize, the presence of heavy sterile neutrinos with some (even very small) mixing to active neutrinos has numerous implications in astrophysics and cosmology. At present, a sterile neutrino with mass of the order of a few $\mathrm{keV}$ and very small mixing $\left(\sin ^{2} 2 \theta \approx 10^{-8}\right)$ with an active one seems to be allowed by constraints, could account for all or some fraction of the dark matter in the Universe, would affect emission of supernovae neutrinos, could explain the pulsar kicks and might lead to observable contributions to $\mathrm{X}$-ray measurements. In the future, more and more precise cosmological observations can constrain very strongly the parameter space for such sterile neutrinos, potentially closing the window or leading to detection of some signal. 


\section{Supersymmetry and neutrinos}

Neutrino masses are not the only motivation to extend the standard model. One also likes to extend it in order to solve the gauge hierarchy problem. Models of low-energy supersymmetry are attractive candidates for the theory of $\mathrm{TeV}$ scale physics. In the minimal supersymmetric extension of the Standard Model (MSSM) neutrinos are massless. Thus, we need to consider supersymmetric extensions of the Standard Model that allow for neutrino masses.

There are basically three questions we like to answer when we talk about the relations between supersymmetry and neutrinos

1. Can successful predictions for neutrino masses of non-supersymmetric extensions of the Standard Model be retained once these models are supersymmetrized? In particular, can supersymmetry help in making such models more motivated?

2. Are there models where neutrino masses arise only due to supersymmetry?

3. Are there interesting phenomena in the slepton sector that can shed light on the issue of neutrino masses, lepton number violation and lepton flavor violation?

The questions in the first item were already discussed in previous sections of this review. Generically, supersymmetry does not upset model predictions regarding neutrinos, and in some cases it in fact helps. For example, in the case of GUT, making the model supersymmetric helps to achieve coupling unification and thus to make the model realistic.

In the following we concentrate on the last two items. We briefly describe two frameworks where neutrino masses are tightly connected to supersymmetry, or more precisely, to supersymmetry breaking, neutrino masses from R-parity violation and from supersymmetry breaking. We then discuss two effects, that of sneutrino oscillation and sneutrino flavor oscillation, that can help us disentangle the origin of neutrino masses using supersymmetric probes. The aspects of supersymmetric seesaw models, i.e. the connection to decays such as $\mu \rightarrow e \gamma$ have been discussed in previous Sections.

\subsection{Neutrino masses from R-parity violation}

Neutrino masses from R-parity violation have been extensively studied. Here we briefly summarize the main results. For a more complete reference list where more details can be found, see, for example, [388].

Once R-parity is violated there is no conserved quantum number that would distinguish between the down-type Higgs doublet and the lepton doublets. (For definitions and notation see, for example, [389].) Thus, these fields in general mix. Such mixings generate neutrino masses; in fact, they generically produce too large masses. One neutrino gets a tree level mass which depends on the mixings between the Higgs and the sneutrinos [390]. The other two neutrinos get their masses at the one loop level, and thus their masses are smaller by, roughly, a loop factor.

There are many different one loop contributions to the neutrino masses. (For a complete list see [391,392].) The "standard" diagrams are those that arise from the R-parity violating trilinear couplings in the superpotential, the so-called $\lambda$ and $\lambda^{\prime}$ couplings. These 
are the only contributions that are present in the supersymmetric limit. Once supersymmetry breaking is included, there are many more contributions (in fact, also the tree level contribution is present only due to supersymmetry breaking). These contribution are likely to be much larger than the $\lambda$ and $\lambda^{\prime}$ loop induced masses. The dominant diagrams are likely to be those that arise due to bilinear couplings [388]. These are the couplings that mix the scalar components of the Higgs and the neutrino superfields. The basic reason that the $\lambda$ and $\lambda^{\prime}$ loop induced masses are very small is that they are proportional to the small down-type quark or charged lepton Yukawa couplings. This suppression factor is absent in the bilinear induced masses.

The most attractive feature of R-parity violation models of neutrino masses is that they naturally generate hierarchical neutrino masses with large mixing angles. This is due to the fact that only one neutrino gets a mass at tree level, while the other neutrinos only acquire loop induced masses. Numerically, however, the predicted mass hierarchy is in general somewhat too strong. Models with R-parity violation also predict observable lepton-number violating processes at possibly observable rates (e.g., [371, 376, 393]).

The biggest puzzle posed by R-parity violation models is to understand the smallness of the neutrino masses. There must be a mechanism that generates very small R-parity violating couplings. There are several ideas of how to do it. For example, the small R-parity violation couplings can be a result of an Abelian horizontal symmetry [394].

\subsection{Neutrino masses from supersymmetry breaking}

The smallness of neutrino masses can be directly related to the mechanism of supersymmetry breaking, in particular, to the mechanism that ensures a weak scale $\mu$ parameter $[231,232,395-398]$. In general, there is no reason why the MSSM $\mu$ parameter is of the order of the weak scale. Generically, it is expected to be at the cut-off scale of the theory, say the Planck or the GUT scale. Phenomenologically, however, $\mu$ is required to be at the weak scale. One explanation, which is known as the Giudice-Masiero mechanism, is that a $\mu$ term in the superpotential is not allowed by a global symmetry. The required effective weak scale $\mu$ is generated due to supersymmetry breaking effects.

The Giudice-Masiero mechanism can be generalized to generate small neutrino masses. It might be that the large Majorana mass term that drives the seesaw mechanism is forbidden by a global symmetry. Effective Majorana mass terms for the right-handed neutrinos, of the order of the weak scale, are generated due to supersymmetry breaking. The same global symmetry can also suppress the Dirac mass between the right and lefthanded neutrinos. Then, the left-handed neutrinos have very small Majorana or Dirac masses as desired.

The emerging neutrino spectrum depends on the exact form of the global symmetry that is used to implement the Giudice-Masiero mechanism. Nevertheless, the feature that the left-handed neutrino masses are very small is generic.

\subsection{Sneutrino oscillation}

As already discussed in this report, it is interesting to find out whether neutrinos have

Majorana masses. In other words, we would like to find out if total lepton number is 
violated in nature. As already discussed, the most promising way to do it, is to look for neutrinoless double beta decay. If supersymmetry is realized in nature we may have other probes. Here we describe one example, that of sneutrino-anti-sneutrino mixing and oscillation [399].

Consider a supersymmetric extension of an extended Standard Model that contains Majorana neutrino masses. For simplicity we consider only one neutrino generation. In such models, the effect of $\Delta L=2$ operators is to introduce a mass splitting and mixing into the sneutrino-anti-sneutrino system. This phenomena is analogous to the effect of a small $\Delta B=2$ perturbation to the leading $\Delta B=0$ mass term in the $B$-system which results in a mass splitting between the heavy and light neutral $B$ mesons. The very small mass splitting can be measured by observing flavor oscillations. The flavor is tagged in $B$-decays by the final state lepton charge. Since $\Delta m_{B} \sim \Gamma_{B}$, there is time for the flavor to oscillate before the meson decays.

The sneutrino system can exhibit similar behavior. The lepton number is tagged in sneutrino decay using the charge of the outgoing lepton. The relevant scale is the sneutrino width. If the sneutrino mass splitting is large, namely when

$$
x_{\tilde{\nu}} \equiv \frac{\Delta m_{\tilde{\nu}}}{\Gamma_{\tilde{\nu}}} \gtrsim 1
$$

and the sneutrino branching ratio into final states with a charged lepton is significant, then a measurable same sign dilepton signal is expected. Any observation of such oscillation will be an evidence for total lepton number violation, namely for Majorana neutrino masses.

The size of the same sign lepton signal is model dependent. It is generically expected that the sneutrino mass splittings are of the order of the neutrino masses, as both are related to $\Delta L=2$ operators. The sneutrino width can vary a lot. When two body decay channels are dominant the width is roughly of the order of $\Gamma_{\tilde{\nu}} \sim \alpha M_{\tilde{\nu}} \sim \mathcal{O}(1 \mathrm{GeV})$, and thus $x_{\tilde{\nu}} \ll 1$ and a very small signal is expected. In models where the two body decay channels are forbidden the sneutrino width can be much smaller. For example, in models where the stau is the LSP there can be a situation where the sneutrino has only three body decay channels allowed. Then $x_{\tilde{\nu}}$ may be large enough for the oscillation signal to be observed.

\subsection{Sneutrino flavor oscillation}

As we know there are large mixing angles in the lepton sector. An independent probe of lepton flavor violation can be provided in supersymmetric models via the slepton sector [400-403]. While generally the slepton mixings are not directly related to the lepton mixings, both are lepton flavor violating effects.

Slepton flavor oscillations arise if the sleptons mass eigenstates are not flavor eigenstates. Experimentally, the signal consists of a lepton flavor imbalance. For example, in a linear collider one can search for a signal like $e^{+} e^{-} \rightarrow \tilde{\nu} \tilde{\nu}^{*} \rightarrow X e^{+} \mu^{-}$, where $X$ is the non leptonic part of the final state. In hadron colliders, one can look for signals like $\chi^{-} \rightarrow \tilde{\nu} \mu^{-} \rightarrow X e^{+} \mu^{-}$, where $\chi^{-}$is a chargino. The oscillation probabilities depend on the slepton mass splittings and their mixing angles. In principle, oscillation signals can 
be used to measure these parameters. Even if this cannot be achieved in practice, just the observation of an oscillation signal will provide an independent confirmation for lepton flavor non-conservation.

In many proposals for supersymmetry breaking, a high degree of degeneracy among sleptons is predicted. As a result, there is the potential for large flavor mixing among the sleptons. This can lead to substantial and observable flavor violating signals at colliders. To be readily observable, it is necessary that mass splittings between the states are not much smaller than the decay widths, and that the mixing angles are not very small. In a large class of supersymmetry breaking models, the splittings can be comparable or even larger than the widths, and the mixing angles may be large. In such cases, dramatic collider signatures are possible. 


\section{Expectations in Superstring Constructions}

There has been relatively little work on the implications of superstring theories for neutrino masses. However, it is known that some of the ingredients employed in grand unified theories and other four-dimensional models may be difficult to implement in known types of constructions. For example, the chiral supermultiplets that survive in the effective four-dimensional field theory are generally bi-fundamental in two of the gauge group factors (including the case of fundamental under one factor and charged under a $U(1))$ for lowest level heterotic constructions, or either bi-fundamental or adjoint for intersecting brane constructions. This makes it difficult to break the GUT symmetry, and even more so to find the high-dimensional Higgs representations (such as the $\mathbf{1 2 6}$ of $S O(10)$ ) usually employed in GUT models for neutrino and other fermion masses. Thus, it may be difficult to directly embed many of the models, especially GUT models involving high-dimensional representations rather than higher-dimensional operators, in a string framework. Perhaps more likely is that the underlying string theory breaks directly to an effective four-dimensional theory including the Standard Model and perhaps other group factors [404]. Some of the aspects of grand unification, especially in the gauge sector, may be maintained in such constructions. However, the GUT relations for Yukawa couplings are often not retained [405-407] because the matter multiplets of the effective theory may have a complicated origin in terms of the underlying string states. Another difference is that Yukawa couplings in string derived models may be absent due to symmetries in the underlying string construction, even though they are not forbidden by any obvious symmetries of the four-dimensional theory, contrary to the assumptions in many non-string models. Finally, higher-dimensional operators, suppressed by inverse powers of the Planck scale, are common.

Much activity on neutrino masses in string theory occurred following the first superstring revolution. In particular, a number of authors considered the implications of an $E_{6}$ subgroup of the heterotic $E_{8} \times E_{8}$ construction [405]- [410]. Assuming that the matter content of the effective theory involves three 27's, one can avoid neutrino masses altogether by fine-tuned assumptions concerning the Yukawa couplings [405]. However, it is difficult to implement a canonical type I seesaw. Each 27 contains two Standard Model singlets, which are candidates for right-handed neutrinos, and for a field which could generate a large Majorana mass for the right-handed neutrinos if it acquires a large vacuum expectation value and has an appropriate trilinear coupling to the neutrinos. However, there are no such allowed trilinear couplings involving three 27's (this is a reflection of the fact that the $\mathbf{2 7}$ does not contain a $\mathbf{1 2 6}$ of the $S O(10)$ subgroup). $E_{6}$ string-inspired models were constructed to get around this problem by invoking additional fields not in the $\mathbf{2 7}[26,407]$ or higher-dimensional operators [410], typically leading to extended versions of the seesaw model involving fields with masses/vevs at the $\mathrm{TeV}$ scale.

Similarly, more recent heterotic and intersecting brane constructions, e.g., involving orbifolds and twisted sectors, may well have the necessary fields for a type I seesaw, but it is again required that the necessary Dirac Yukawa couplings and Majorana masses for the right-handed neutrinos be present simultaneously. Dirac couplings need not emerge at the renormalizable level, but can be of the form

$$
\left\langle S_{1}^{\prime} \cdots S_{d-3}^{\prime}\right\rangle N L H_{u} / M_{\mathrm{PL}}^{d-3},
$$


where the $S_{i}^{\prime}$ are standard model singlets which acquire large expectation values $(d=3$ corresponds to a renormalizable operator). Similarly, Majorana masses can be generated by the operators

$$
\left\langle S_{1} \cdots S_{n-2}\right\rangle N N / M_{\mathrm{PL}}^{n-3} .
$$

Whether such couplings are present at the appropriate orders depends on the underlying string symmetries and selection rules, which are often very restrictive. It is also necessary for the relevant $S$ and $S^{\prime}$ fields to acquire the needed large expectation values, presumably without breaking supersymmetry at a large scale. Possible mechanisms involve approximately flat directions of the potential, e.g., associated with an additional $U(1)^{\prime}$ gauge symmetry [95,411], stringy threshold corrections $[412,413]$, or hidden sector condensates [414].

There have been surprisingly few investigations of neutrino masses in explicit semirealistic string constructions. It is difficult to obtain canonical Majorana masses in intersecting brane constructions [415], because there are no interactions involving the same intersection twice. Two detailed studies [416,417] of nonsupersymmetric models with a low string scale concluded that lepton number was conserved, though a small Dirac mass might emerge from a large internal dimension. Large enough internal dimensions for the supersymmetric case may be difficult to achieve, at least for simple toroidal orbifolds.

There are also difficulties for heterotic models. An early study of $Z_{3}$ orbifolds yielded no canonical Dirac neutrino Yukawas [418] at low order. Detailed analyses of free fermionic models and their flat directions were carried out in $[414,419]$ and $[420,421]$. Both studies concluded that small Majorana masses could be generated if one made some assumptions about dynamics in the hidden sector. In $[414,419]$ the masses were associated with an extended seesaw involving a low mass scale. The seesaw found in $[420,421]$ was of the canonical type I type, but in detail it was rather different from GUT-type models. A seesaw was also claimed in a heterotic $Z_{3}$ orbifold model with $E_{6}$ breaking to $S U(3) \times S U(3) \times S U(3)$ [422]. A recent study of $Z_{6}$ orbifold constructions found Majorana-type operators [423], but (to the order studied) the $S_{i}$ fields did not have the required expectation values.

In [424] a large class of vacua of the bosonic $Z_{3}$ orbifold were analyzed with emphasis on the neutrino sector to determine whether the minimal type I seesaw is common, or if not to find possible guidance to model building, and possibly to get clues concerning textures and mixing if examples were found. Several examples from each of 20 patterns of vacua were studied, and the nonzero superpotential terms through degree 9 determined. There were huge number of $D$ flat directions, with the number reduced greatly by the $F$ flatness condition. Only two of the patterns had Majorana mass operators, while none had simultaneous Dirac operators of low enough degree to allow neutrino masses larger than $10^{-5} \mathrm{eV}$. (One apparently successful model was ruined by off-diagonal Majorana mass terms.) It is not clear whether this failure to obtain a minimal seesaw is a feature of the particular class of construction, or whether it is suggesting that stringy constraints and selection rules might make string vacua with minimal seesaws rare. Systematic analyses of the neutrino sector of other classes of constructions would be very useful.

There are other possibilities for obtaining small neutrino masses in string constructions, such as extended seesaws and small Dirac masses from higher dimension operators. Small Dirac neutrino masses from type I anisotropic compactifications have been dis- 
cussed recently in [425]. The possibility of embedding type II seesaw ideas (involving Higgs triplets) in string constructions was considered in [426]. It is possible to obtain a Higgs triplet of $S U(2)$ with non-zero hypercharge in a higher level construction (in which $S U(2) \times S U(2)$ is broken to a diagonal subgroup). In this case, because of the underlying $S U(2) \times S U(2)$ symmetry the Majorana mass matrix for the light neutrinos should involve only off-diagonal elements (often with one of the three off-diagonal elements small or vanishing), with profound phenomenological consequences, including an inverted hierarchy and two large mixings. This is a top-down motivation for the texture $\mathrm{C}$ in Table 2 . 


\section{Theories with a TeV-scale $U(1)^{\prime}$}

Many extensions of the Standard Model and MSSM include the existence of additional non-anomalous $U(1)^{\prime}$ gauge symmetries. These include many superstring constructions [427], grand unified theories, Little Higgs models, and models of dynamical symmetry breaking (DSB) [428]. In a regular grand unified theory the $U(1)^{\prime}$ breaking needs to be at a large scale, because scalars that can mediate proton decay can have masses no larger than the $U(1)^{\prime}$ breaking scale. In string theories, the $U(1)^{\prime}$ symmetry breaking is usually induced by soft supersymmetry breaking effects at the TeV scale $[427,429,430]$, although in some cases there is the possibility of breaking along an $F$ and $D$ flat direction at an intermediate scale [411] (depending on the sign of a combination of soft mass-squares). The Little Higgs and DSB models are at the TeV scale.

A TeV scale $Z^{\prime}$ has many interesting phenomenological consequences [431], but here we are concerned with neutrino masses. In the intermediate $Z^{\prime}$-scale case, higher-dimensional operators, involving one or more powers of the fields with intermediate-scale vevs but suppressed by powers of the Planck mass, can yield naturally small Dirac neutrino masses [95, 411]. Variants can also lead to mixing between light ordinary and sterile neutrinos, as suggested by the LSND results, or even to a type I seesaw.

Models in which the $U(1)^{\prime}$ breaking is at the TeV scale generally do not allow a canonical type I seesaw model, because the Majorana mass for the right-handed neutrino $N_{R}$ requires $U(1)^{\prime}$ breaking (unless the $N_{R}$ carries no $U(1)^{\prime}$ charge). However, a number of other possibilities are allowed for the neutrino masses [432], including small Dirac masses (e.g., associated with a second $U(1)^{\prime}$ broken at an intermediate scale), and Majorana masses associated with a TeV-scale seesaw [26] or a heavy Higgs triplet (Type II seesaw) [226]. The small Dirac mass case involves a strong constraint from Big Bang Nucleosynthesis, because the $Z^{\prime}$ interactions could efficiently produce the right-handed components prior to nucleosynthesis, leading to too much ${ }^{4} \mathrm{He}$ [433]. For generic couplings of the $Z^{\prime}$ to the $N_{R}$ the observed abundance implies a $Z^{\prime}$ mass larger than around 4 $\mathrm{TeV}$, stronger than indirect or collider constraints [434]. This can be evaded or weakened if the $N_{R}$ carries no $U(1)^{\prime}$ charge (as can occur naturally in some models involving two $U(1)^{\prime}$ factors $\left.[431,432]\right)$ or if the mass is Majorana. 


\section{Neutrino Masses in Theories with Dynamical Sym- metry Breaking}

The source of electroweak symmetry breaking (EWSB) remains unknown, and a dynamical origin of this breaking is an appealing possibility. This can be realized via the formation of a bilinear condensate involving fermions with a new strong gauge interaction, generically called Technicolor (TC) $[435,436]$. Indeed, one may recall that in both of the well-known two cases in which scalar fields have been used in models of spontaneous symmetry breaking, namely the Ginzburg-Landau effective Hamiltonian for superconductivity and the Gell-Mann Levy sigma model for hadronic chiral symmetry breaking, the scalar fields were not fundamental, and the true underlying physics responsible for these respective phenomena involved the formation of bilinear fermion condensates (Cooper pairs in superconductivity and the $\langle\bar{q} q\rangle$ condensate in QCD). In order to communicate this symmetry breaking in the Technicolor sector to the standard-model (technisinglet) fermions, one embeds the Technicolor model in a larger, extended Technicolor (ETC) theory $[437,438]$. To satisfy constraints from flavor-changing neutral-current processes, the ETC vector bosons that mediate generation-changing transitions must have large masses. These masses arise from the sequential breaking of the ETC gauge symmetry on mass scales ranging from $10^{3} \mathrm{TeV}$ down to the TeV level. Precision measurements place tight constraints on these models, suggesting that there are a small number of new degrees of freedom at the $\mathrm{TeV}$ scale and that the Technicolor theory has a slowly running ("walking") gauge coupling with large anomalous dimensions [439].

Since ETC models do not involve any superheavy GUT-scale mass, there was for a long time a puzzle of how one could explain light neutrino masses in these models. A possible solution to this puzzle was given in Ref. [92] and studied further in Refs. [93, 441, 442]. This does involve a seesaw, but one of a new type not involving any superheavy GUT scale. The resultant formula $M_{\nu} \simeq\left(M_{\nu}^{D}\right)^{2} / m_{R}$ holds, with the largest Dirac neutrino masses of order a few keV and the relevant Majorana neutrino mass of order $\mathrm{O}(0.1) \mathrm{GeV}$ to $\mathrm{O}(100) \mathrm{GeV}$. These Dirac and Majorana neutrino masses are greatly suppressed relative to conventional values. This suppression is a natural consequence of the representations of the ETC gauge group for the various neutrino fields. These ETC models are not yet developed sufficiently to make detailed predictions for leptonic mixing angles, but it seems possible to get substantial neutrino mixing. One interesting feature of this mechanism for neutrino masses is that there are only two, rather than three right-handed electroweaksinglet neutrinos, in contrast, e.g., to $S O(10)$ GUT models.

The ETC gauge group $\mathrm{SU}\left(N_{E T C}\right)$ commutes with the Standard Model (SM) group $G_{S M}$. The ETC group gauges the three generations of technisinglet fermions and connects them with the technicolored fermions. The ETC gauge symmetry is chiral, so that when it becomes strong, sequential breaking occurs naturally. The ETC symmetry breaking takes place in stages, leaving the residual exact technicolor gauge symmetry $\mathrm{SU}\left(N_{T C}\right)$. This entails the relation $N_{E T C}=N_{\text {gen }}+N_{T C}=3+N_{T C}$, where $N_{\text {gen }}$ is the number of standard-model fermion generations. The choice of $N_{T C}=2$ is required for the mechanism of Ref. [92] to work. This thus implies $N_{E T C}=5$; i.e., one uses an $S U(5)_{E T C}$ gauge theory. A related $S U(5)_{E T C}$ theory had previously been studied in Ref. [440]. The choice $N_{T C}=2$ 
has two other motivations: (a) it minimizes the TC contributions to the electroweak $S$ parameter, which is a stringent constraint on TC theories, (b) with a standard-model family of technifermions, $Q_{L}=\left(\begin{array}{c}U \\ D\end{array}\right)_{L}, L_{L}=\left(\begin{array}{c}N \\ E\end{array}\right)_{L}, U_{R}, D_{R}, N_{R}, E_{R}$ transforming according to the fundamental representation of $\mathrm{SU}(2)_{T C}$, it can yield an approximate infrared fixed point and the associated walking behavior. This sequential breaking of the $S U(5)_{E T C}$ is driven by the condensation of SM-singlet fermions.

One can explore whether this dynamical neutrino mass mechanism could take place in the context of ETC theories in which the strong-electroweak gauge group is extended beyond that of the Standard Model. Theories with the left-right symmetry group $G_{L R}=$ $\mathrm{SU}(3)_{c} \times \mathrm{SU}(2)_{L} \times \mathrm{SU}(2)_{R} \times \mathrm{U}(1)_{B-L}[72]$ and the group $G_{422}=\mathrm{SU}(4)_{P S} \times \mathrm{SU}(2)_{L} \times \mathrm{SU}(2)_{R}$ [443] are of particular interest here, where $B$ and $L$ denote baryon and lepton number and $S U(4)_{P S}$ combines color and lepton number. Ref. [93] presented a full ETC model in which these extended strong-electroweak gauge symmetries can be broken dynamically and showed that the mechanism of Ref. [92] can also hold here. Dynamical symmetry breaking of $G_{L R}$ has also been studied in Ref. [444]. Further, dynamical symmetry breaking of the electroweak symmetry can be triggered by a neutrino condensate [445]. ETC theories have many other testable implications. Some recent work has focused on ETC contributions to dimension-5 dipole moment operators $[148,149]$ and dimension- 6 four-fermion operators and their effects [442]. 


\section{Neutrinos in extra dimensions}

The pioneering idea by Kaluza and Klein (KK) [447] that our world may have more than four dimensions has attracted renewed interest over the last ten years [448-451]. The possible existence of extra dimensions has enriched dramatically our perspectives in searching for physics beyond the Standard Model. Obviously, extra dimensions have to be sufficiently compact to explain why they have escaped detection so far, although their allowed size is highly model-dependent [452]. This means that the derived constraints not only depend on the number of the fields sensitive to extra dimensions and their transformation properties with respect to those, but also on the geometry and/or the shape of the new dimensions. In the latter case, higher-dimensional theories may be distinguished between those formulated on a flat space and those that utilize a warped geometry.

Higher-dimensional theories may also provide interesting alternatives for explaining the smallness of the observed light neutrino masses. Their predictions for the lightneutrino spectrum can be confronted with recent neutrino oscillation data. In the following, we discuss a generic higher-dimensional neutrino scenario in which a flat geometry is realized.

The original formulation of higher-dimensional neutrino models $[453,454]$ relies on the possible existence of singlet neutrinos that propagate in a higher $[1+(3+\delta)]$-dimensional space which is usually termed the bulk, where $\delta$ is the number of the additional spatial compact dimensions. In this formulation, the ordinary SM particles reside in a $(1+$ 3)-dimensional Minkowski subspace, which is called the wall. Hence, the left-handed neutrinos and the Higgs bosons live on the wall. The overlap of their wave-functions with the bulk neutrinos is suppressed by the volume of the extra-dimensional space $\left(R M_{F}\right)^{\delta / 2} \approx$ $M_{\mathrm{P}} / M_{\mathrm{F}}$, where $R$ is the common compactification radius, $M_{\mathrm{F}}$ is the fundamental gravity scale and $M_{\mathrm{P}} \approx 10^{16} \mathrm{TeV}$ is the usual Planck mass. This volume-suppression factor gives rise to effective neutrino Yukawa couplings that are naturally very small, i.e. of order $M_{\mathrm{F}} / M_{\mathrm{P}} \sim 10^{-15}$, for $M_{\mathrm{F}}=10 \mathrm{TeV}$, although the original higher-dimensional Yukawa couplings of the theory could be of order unity. This suppression mechanism $[453,454]$ is a generic feature of these higher-dimensional neutrino models realized on an toroidal bulk; it has some dependence on the compactification properties of the bulk neutrinos and the structure of the Higgs sector of the theory [455, 456].

To illuminate the discussion that follows, we consider a minimal extension of the SM where one 5-dimensional (bulk) sterile neutrino $N$ has been added. Furthermore, the extra dimension, say $y$, is compactified on a $S^{1} / Z_{2}$ orbifold. The SM fields are localized on a 4-dimensional Minkowski subspace at the orbifold fixed point $y=0$. Different minimal models may be constructed depending on the way that lepton number is broken:

(i) One may add lepton-number violating bilinears of the Majorana type in the Lagrangian [453], e.g. operators of the form $N^{T} C^{(5)-1} N$, where $C^{(5)}=-\gamma_{1} \gamma_{3}$ is the charge conjugation operator.

(ii) Lepton-number-violating mass terms can be generated through the Scherk-Schwartz mechanism [457]. This mechanism turns out to be equivalent to (i), after KK reduction. 
(iii) Lepton number can be broken if the $Z_{2}$-even and $Z_{2}$-odd two-component spinors of the bulk neutrino couple simultaneously to the same left-handed charged lepton state. This is only possible if the 3-brane describing our observable world is shifted from the $S^{1} / Z_{2}$ orbifold fixed point $[453,458]$.

(iv) Violation of the lepton number can be achieved by introducing operators of higher dimension in the number of fields, e.g. $(L \Phi)^{2} / M_{\mathrm{F}}$. Such operators may be generated through gravity effects [459].

The current neutrino oscillation data provide an important test for singling out a good candidate model that includes higher-dimensional neutrinos. For example, orbifold models with one bulk neutrino [453,455,459-463], based on models of type (i) and/or (ii) mentioned above, seem to prefer the Small Mixing Angle (SMA) solution which is highly disfavored by recent neutrino data analyses. Alternatively, if all neutrino data are to be explained by oscillations of active neutrinos with a small admixture of a sterile KK component, then the compactification scale has to be much higher than the brane-Dirac mass terms. After integrating out the bulk neutrino of the model, the resulting effective light-neutrino mass matrix is of rank 1. Because of this restricted form of the neutrino mass matrix, two out of the three active neutrinos are massless. Since only one neutrinomass difference can be formed in this case, it proves difficult to accommodate all neutrino oscillation data in these models [461-463].

\subsection{Three bulk neutrinos}

One way to avoid this problem is to add three bulk neutrinos, one for each generation [460, 463]. This model, in the absence of CP phases, is characterized by seven parameters: three neutrino masses $m_{1,2,3}$ and three mixing angles for left handed neutrinos as defined earlier and the radius of the large extra dimension. Since the three mixing angles are arbitrary, the model can easily accommodate the bilarge mixing solution preferred by oscillation data. In the diagonal mass basis, the bulk neutrinos are associated with mass eigenstates. The mixing of the $i$ th active neutrino with the nth KK mode of the corresponding bulk neutrino is given by $\xi_{i, n} \simeq m_{i} R / n$. It is interesting that all mixings are intimately connected with the masses. There are limits on $\xi_{i}$ from laboratory data such as CHOOZPalo-Verde as well as from big bang nucleosynthesis [464]. BBN constraints for one extra dimension give $\xi_{3}^{2} \leq 1.7 \times 10^{-4}(\mathrm{eV} . R)^{0.92}$. For a hierarchical pattern, using $\xi=m_{3} R$, one gets $R \leq 0.03 \mathrm{eV}^{-1}$. The bounds from neutrino data such as solar and atmospheric etc. are less stringent [463] and are roughly given by: $R \leq 4 \mathrm{eV}^{-1}$.

Among the consequences of this model, two are especially interesting. Both of these concern the KK tower of sterile neutrinos.

(i) In the presence of the infinite tower of states, the magnetic moment of the neutrino gets contribution from all the states [465]. For instance in the scattering of a neutrino of energy $E \approx 10 \mathrm{MeV}$ (corresponding to a reactor neutrino beam) the number of states contributing to the magnetic moment is given by $(E R)^{2} \sim 10^{18}$. Since all the states add incoherently, the effective magnetic moment is increased from $10^{-20} \mu_{B}$ to $10^{-11} \mu_{B}\left(\mu_{B}\right.$ is the Bohr magneton). The effect on the differential cross section $d \sigma / d T$, where $T$ is the electron recoil energy has recently been calculated and given in Fig. 12.1] [466]. 


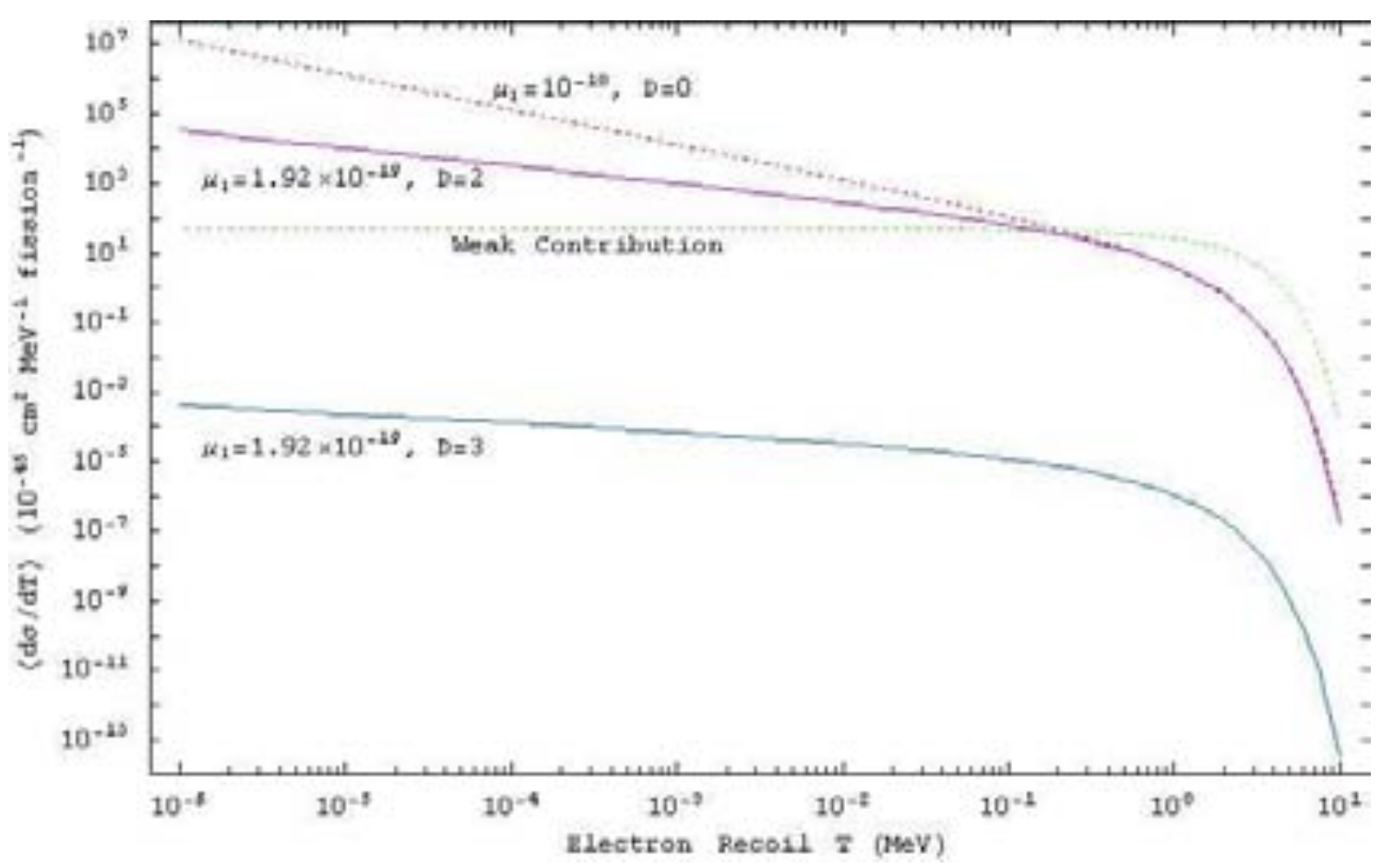

Figure 21: The figures give the contribution of neutrino magnetic moment for the case of single Dirac neutrino, for two large extra dimensions (and a comparison between the two) to differential cross section $\frac{d \sigma}{d T}$ (where $T$ is the electron recoil energy) for neutrino electron scattering and compares it with the case of one right-handed neutrino ("Standard Model with one right-handed neutrino").

(ii) A second consequence of the existence of the KK tower of sterile neutrinos is the possibility that when neutrinos travel through dense matter there can be MSW resonances [455] and give rise to a dip pattern $[455,467]$ in the neutrino survival probability corresponding to energies spaced by $E \approx \Delta m_{\nu_{F} \nu_{K}}^{2} / 2 \sqrt{2} G_{F} N_{e}$ (i.e. $E, 4 E, 9 E, \ldots$ ). The dip arises because typically the survival probability goes like $e^{-c \frac{\Delta m^{2}}{E}}$. Therefore at lower energies, there is more suppression which with increase in energy becomes less and less effective. Also the resonance condition is not satisfied as energy increases, if it was satisfied at lower energies. For the solar neutrinos, such dip structure is quite pronounced [467]. In the hierarchical pattern for neutrino masses, this would correspond to $E \approx 10 \mathrm{MeV}$ for densities comparable to solar core. The value of the energy clearly depends on the size of the extra dimensions, growing with $R^{-1}$. This is a very interesting phenomenon which could be used to probe this class of extra dimension models.

\subsection{Lepton number breaking in the bulk}

In the three bulk neutrino picture, all the neutrinos are Dirac neutrinos since the model has an additional global $B-L$ symmetry. An interesting possibility is the scenario 


\begin{tabular}{|cccc|}
\hline Observable & $\begin{array}{c}h_{e}=h_{\mu}=h_{\tau}=h \underset{\sim}{z} \\
\text { Lower limit on } M_{F} / h^{2}[\mathrm{TeV}] \\
\delta=2\end{array}$ & $\delta=3$ & $\delta=6$ \\
\hline $\operatorname{BR}(\mu \rightarrow e \gamma)$ & 75 & 43 & 33 \\
\hline $\operatorname{BR}(\mu \rightarrow e e e)$ & 250 & 230 & 200 \\
\hline $\operatorname{BR}\left(\mu_{22}^{48} \mathrm{Ti} \rightarrow e_{22}^{48} \mathrm{Ti}\right)$ & 380 & 320 & 300 \\
\hline
\end{tabular}

Table 7: One-loop-level limits on $M_{F} / h^{2}$ from [468].

(iii) described above, where sizable lepton-number violation is induced by shifting the $y=0$ brane by an amount $a \sim 1 / M_{W}$. In this scenario, the tree-level rank-1 form of the effective neutrino mass matrix can be significantly modified through lepton-number violating Yukawa terms, thus offering sufficient freedom to describe the neutrino oscillation data [458].

In addition to constraints from neutrino oscillation data, other experiments can also play an important role in constraining higher-dimensional neutrino models. Specifically, strong limits on $M_{F}$ and the Yukawa couplings of the theory may be obtained from the non-observation of lepton-flavor-violating decays in muon and tau decays and from the absence of $\mu$-e conversion in nuclei $[468,469]$. Table 7 gives a brief summary of these limits. These phenomenological constraints are complementary to those obtained from pure theoretical considerations, such as perturbative unitarity [470].

Another low-energy experiment of great importance is the neutrinoless double beta decay of a nucleus. The recently claimed experimental evidence [31] of an effective neutrino mass of order $0.4 \mathrm{eV}$ (see however [32]), combined with information from solar and atmospheric neutrino data, restricts the admissible forms of the light-neutrino mass hierarchies in 4-dimensional models with 3 left-handed (active) neutrinos. The allowed scenarios contain either degenerate neutrinos or neutrinos that have an inverse mass hierarchy [471]. A positive interpretation of the recently reported $0 \nu \beta \beta$ signal [31] imposes additional constraints on model-building. For example, higher-dimensional models that utilize the shining mechanism from a distant brane [472] may accommodate an effective neutrino mass of $0.4 \mathrm{eV}$ but also predict the emission of Majorons. On the other hand, 5-dimensional models formulated on a warped geometric space [473] face difficulties to account for the observable excess in [31].

In the context of $S^{1} / Z_{2}$ orbifold models, one has to solve an additional theoretical problem. The resulting KK neutrinos group themselves into approximately degenerate pairs of opposite CP parities. Because of this, the lepton-number-violating KK-neutrino effects cancel each other leading to unobservably small predicted values for the $0 \nu \beta \beta$ decay. These disastrous CP parity cancellation effects can be avoided by arranging the opposite CP parity KK neutrinos to couple to the $W^{ \pm}$bosons with unequal strength. This feature can naturally be implemented if the $y=0$ wall displaced from one of the $S^{1} / Z_{2}$ orbifold fixed points by an amount of order $1 / M_{W}$. A unique prediction of such a model [458] is that the effective neutrino mass and the scale of the light neutrino masses can be completely decorrelated. 


\section{Other new physics and neutrinos}

\subsection{New long range forces}

Long range forces in the context of particle physics originated with the ideas of Yang and Lee [474] and Okun [475] who proposed that gauging the baryon number or lepton number would give rise to a composition dependent long range force which could be tested in the classic Eotovos type experiments [476]. A special class of long range forces which distinguish between leptonic flavors have far reaching implications for the neutrino oscillations $[477,478]$ which may be used as a probe of such forces.

The standard model Lagrangian is invariant under four global symmetries corresponding to the Baryon and three Lepton numbers $L_{\alpha}(\alpha=e, \mu, \tau)$. Of these, only three combinations [479] of lepton numbers (i) $L_{e}-L_{\mu}$, (ii) $L_{e}-L_{\mu}$ or (iii) $L_{\mu}-L_{\tau}$, can be gauged in an anomaly free way without extending the matter content. The existence of neutrino oscillations implies that these symmetries have to be broken but the relevant gauge bosons can still be light if the corresponding couplings are very weak. It is possible in this case to obtain light gauge boson induced forces having terrestrial range (e.g. the Sun-Earth distance) without invoking extremely low mass scales [477]. The exchange of such boson would induce matter effects in terrestrial, solar and atmospheric neutrino oscillations. For example, the electrons inside the sun generate a potential $V_{L R}$ at the earth surface given by

$$
V_{L R}=\alpha \frac{N_{e}}{R_{e s}} \approx\left(1.04 \times 10^{-11} \mathrm{eV}\right)\left(\frac{\alpha}{10^{-50}}\right)
$$

where $\alpha \equiv \frac{g^{2}}{4 \pi}$ corresponds to the gauge coupling of the $L_{e}-L_{\mu, \tau}$ symmetry,$N_{e}$ is the number of electrons inside the sun and $R_{e s}$ is the earth-sun distance $\approx 7.6 \times 10^{26} \mathrm{GeV}^{-1}$. The present bound on the $Z$-dependent force with range $\lambda \sim 10^{13} \mathrm{~cm}$ is given [476] by $\alpha<3.3 \times 10^{-50}$. Eq. (68) then shows that the potential $V_{L R}$ can introduce very significant matter-dependent effects in spite of the very strong bound on $\alpha$. One can define a parameter

$$
\xi \equiv \frac{2 E_{\nu} V_{L R}}{\Delta m^{2}}
$$

which measures the effect of the long range force in any given neutrino oscillation experiment. Given the terrestrial bound on $\alpha$, one sees that $\xi$ is given by $\xi_{a t m} \sim 27.4$ in atmospheric or typical long-baseline experiments while it is given by $\xi_{\text {solar }} \sim 7.6$ in the case of the solar or KamLAND type of experiments. In either case, the long range force would change the conventional oscillation analysis. Relatively large value of $\alpha$ suppresses the oscillations of the atmospheric neutrinos. The observed oscillations then can be used to put stronger constraints on $\alpha$ which were analyzed in [477]. One finds the improved 90\% CL bound .

$$
\alpha_{e \mu} \leq 5.5 \times 10^{-52} \quad ; \quad \alpha_{e \tau} \leq 6.4 \times 10^{-52},
$$

in case of the $L_{e}-L_{\mu, \tau}$ symmetries, respectively.

Although these bounds represent considerable improvement over the conventional fifth force bound, they still allow interesting effects which can be used as a probe of such long range forces in future long-baseline experiments with super beam or at neutrino factories. As a concrete example, let us consider the influence of the $L_{e}-L_{\mu}$ gauge interactions 
on the long-baseline oscillations of muon neutrinos of $\mathcal{O}(\mathrm{GeV})$ energy. The oscillations of these neutrinos are governed by the following $3 \times 3$ (mass) ${ }^{2}$ matrix in the flavor basis:

$$
M_{\nu}^{2}=U^{*} \operatorname{Diag}\left(m_{1}^{2}, m_{2}^{2}, m_{3}^{2}\right) U^{\dagger}+\operatorname{Diag}\left(A_{C C}+A_{L R},-A_{L R}, 0\right) .
$$

$U$ denotes the (vacuum) mixing matrix for which we adopt the conventional parametrization. $A_{C C}=2 E_{\nu} \sqrt{2} G_{F} n_{e} \approx\left(1.04 \times 10^{-13} \mathrm{eV}\right) 2 E_{\nu}$ describes the conventional MSW matter contribution generated by the earth matter (density $\rho \sim 2.8 \mathrm{gm} / \mathrm{cm}^{3}$; electron fraction $\left.Y_{e} \sim 0.49\right)$. The $A_{L R} \approx\left(1.04 \times 10^{-13} \mathrm{eV}\right) 2 E_{\nu} \alpha_{52}$ with $\alpha_{52}$ denoting the coupling of the long range force measured in units of $10^{-52}$. The $A_{L R}$ term dominates over $A_{C C}$ if $\alpha$ saturates the bound in Eq. (69). The matter induced terms in Eq. (170) modify the neutrino

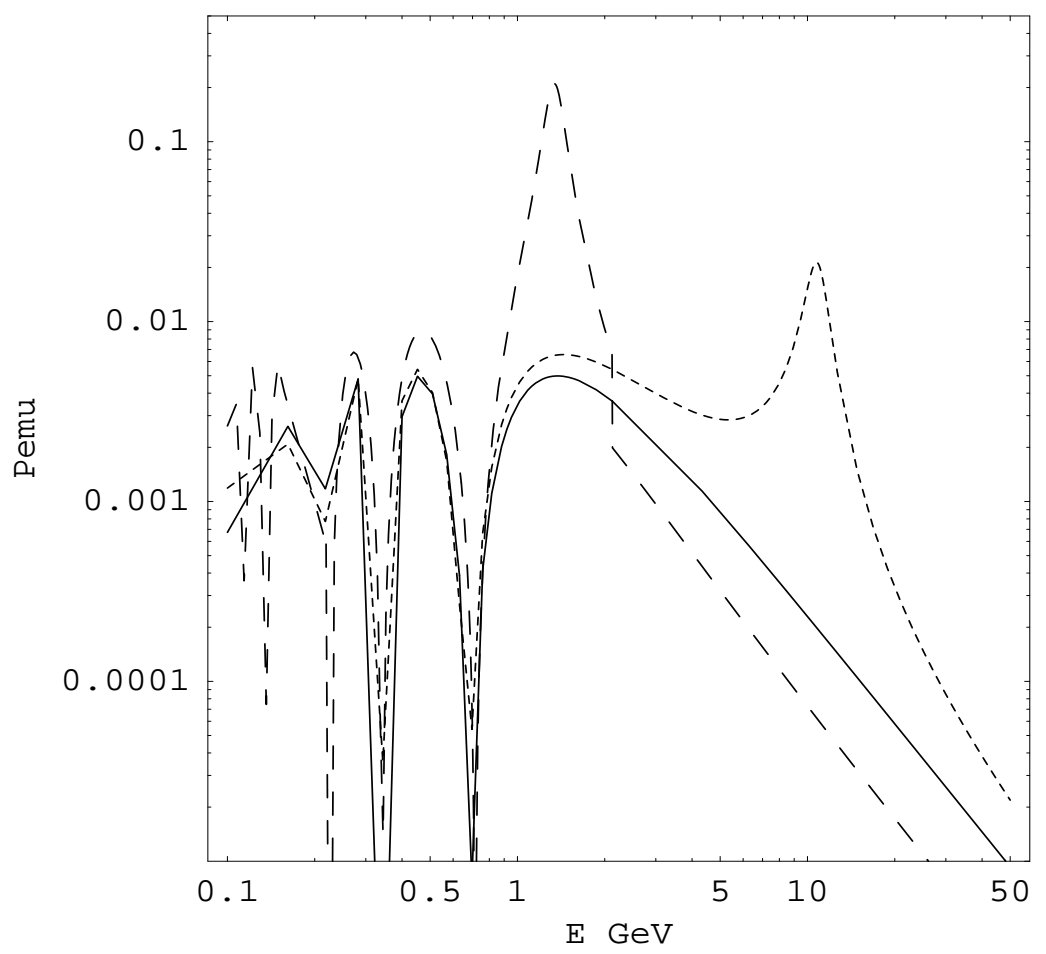

Figure 22: The long-baseline neutrino oscillation probability $P_{e \mu}$ in case of vacuum (solid), earth matter effects (dotted) and with inclusion of the long range potential $V_{L R}$ (dashed). The plotted curves correspond to a baseline of $740 \mathrm{~km}, \Delta m_{32}^{2}=2.5 \times 10^{-5} \mathrm{eV}^{2}, \Delta m_{21}^{2}=7.0 \times 10^{-5}$ $\mathrm{eV}^{2},\left(\theta_{12}, \theta_{23}\right)=\left(32^{\circ}, 45^{\circ}\right), \alpha_{e \mu}=5.5 \times 10^{-52}$ and $\sin \theta_{13}=0.05$.

oscillation in a non-trivial manner. This effect is analyzed in the limit of the vanishing solar scale and $A_{L R}=0$ in [480]. The 23 mixing angle remains unaffected by the matter induced contribution but the 13 angle can get resonantly enhanced for a neutrino energy 
given by

$$
E_{\nu} \approx \frac{\cos 2 \theta_{13} \Delta m_{32}^{2}}{2 \sqrt{2} G_{F} n_{e}} \approx 11.8 \mathrm{GeV} .
$$

This leads to a rise in the oscillation probability $P_{e \mu}$, Figure(22). The additional long range contribution results in a noticeable shift in the resonance energy as seen from Fig. (22). We assumed normal hierarchy in this figure. The resonance behavior would be absent in case of the inverted hierarchy or in case of the anti-neutrino beam. While more detailed study is required to distinguish these cases, it is clear that future observations of matter effects in the long-baseline neutrino experiments provide a good probe of additional long range forces.

\subsection{Lorentz noninvariance, CPT violation and decoherence}

\subsubsection{CPT Violation}

In this section, we discuss neutrino oscillation phenomenology in the presence of CPT violation. CPT is a symmetry in any theory that satisfies the three assumptions that are normally taken for granted: (1) locality, (2) Lorentz invariance, and (3) hermiticity of the Hamiltonian. In particular, it predicts that the mass is common for a particle and its anti-particle. Any violation of CPT therefore would have a profound consequence on fundamental physics.

The best limit on CPT violation is in the neutral kaon system, $\left|m\left(K^{0}\right)-m\left(\bar{K}^{0}\right)\right|<$ $10^{-18} m_{K}=0.50 \times 10^{-18} \mathrm{GeV}$ [481]. Having such a stringent bound does not seem a sizable CPT violation in neutrino at the first sight. However, the kinematic parameter is mass-squared instead of mass, and the constraint may naturally be considered on the CPT-violating difference in mass-squared $\left|m^{2}\left(K^{0}\right)-m^{2}\left(\bar{K}^{0}\right)\right|<0.25 \mathrm{eV}^{2}$. In comparison, the combination of SNO and KamLAND data leads to the constraint $\left|\Delta m_{\nu}^{2}-\Delta m_{\bar{\nu}}^{2}\right|<$ $1.3 \times 10^{-3} \mathrm{eV}^{2}(90 \% \mathrm{CL})$ and hence currently the best limit on CPT violation [482].

Having seen that the CPT violation in neutrino masses may be of size relevant to neutrino oscillation, it is useful to discuss how it may affect the phenomenology. In fact, the primary motivation for recent discussions on CPT violation in neutrino oscillation has been to reconcile LSND data with other data [483]. It is well known that the LSND data is not quite consistent with the other oscillation evidence and limits even if a sterile neutrino state is introduced, both for $2+2$ and $3+1$ spectra (see [349] for a recent analysis; adding more sterile states helps [47]). The main point is that the LSND oscillation is primarily an anti-neutrino oscillation $\bar{\nu}_{\mu} \rightarrow \bar{\nu}_{e}$, while the solar neutrino oscillation is purely neutrinos $\nu_{e} \rightarrow \nu_{\mu, \tau}$. It was shown to fit the LSND, solar, and atmospheric neutrino data simultaneously without invoking a sterile neutrino at that time [483-485]. Phenomenology had been further developed in Refs. [486, 487].

However, KamLAND data shows $\bar{\nu}_{e} \rightarrow \bar{\nu}_{\mu, \tau}$ oscillation with parameters consistent with the solar neutrino oscillation, and the CPT-violation alone cannot explain LSND. A new proposal tried to explain LSND and atmospheric anti-neutrino oscillations with a single $\Delta m^{2}$ [487], which was excluded by a global fit in [488]. Currently the best fit to the data is obtained by allowing for one sterile neutrino and CPT violation [489]. Because the short-baseline experiments that are constraining the interpretation of LSND data with 


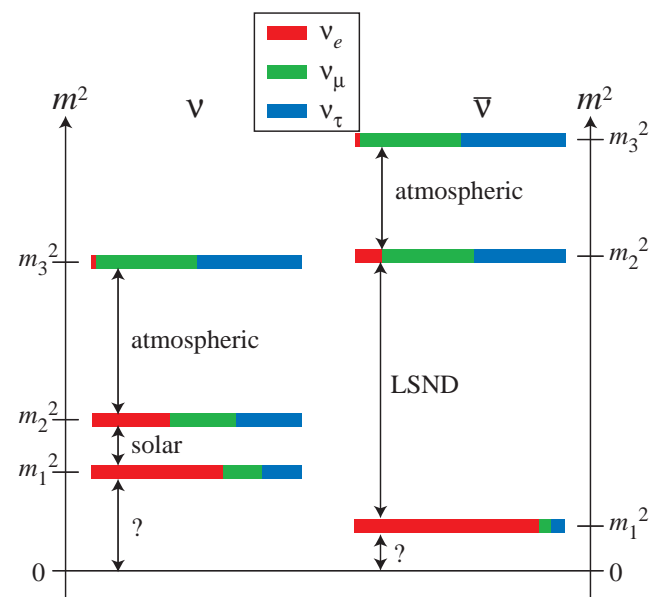

Figure 23: The proposed spectra of neutrinos and anti-neutrinos in [483] and [484]. Excluded by KamLAND.

sterile neutrino are mostly in neutrinos but not in anti-neutrinos, the $3+1$ spectrum is allowed if there is little mixing of the sterile state with others in neutrinos.
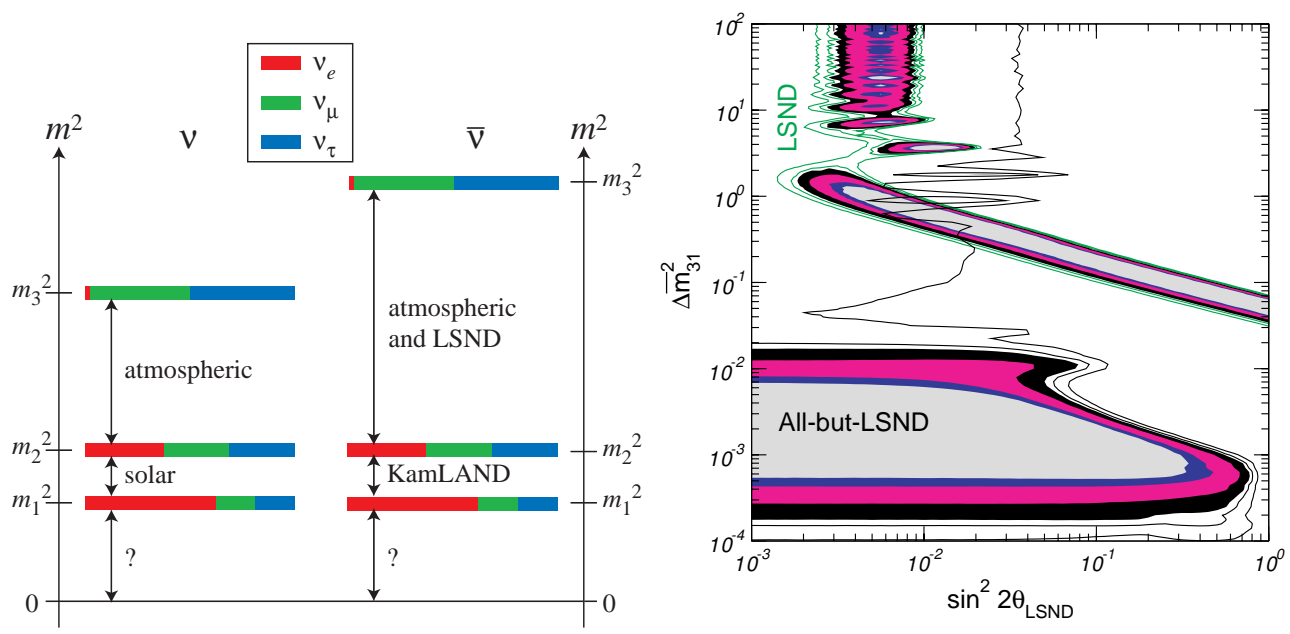

Figure 24: The revised proposal in [487]. Excluded by the analysis taken from [488].

Even though arbitrarily changing the neutrino and anti-neutrino masses seems to preserve Lorentz invariance, interacting theory also violates Lorentz invariance [490]. All discussions above assumed Lorentz invariance and hence should be regarded as purely phenomenological exercise. One theoretically well-defined way to break CPT is to introduce a cosmological "matter effect," namely a background number density coupled to neutrinos. However, such framework does not explain data consistently [491]. See also [492] for a different framework of CPT violation and a recent discussion on the use of decoherence and CPT violation [493]. Lorentz invariance violation in the neutrino sector can arise via the see-saw mechanism. As discussed in [494] this could explain why it would not be seen in the charged lepton sector. 


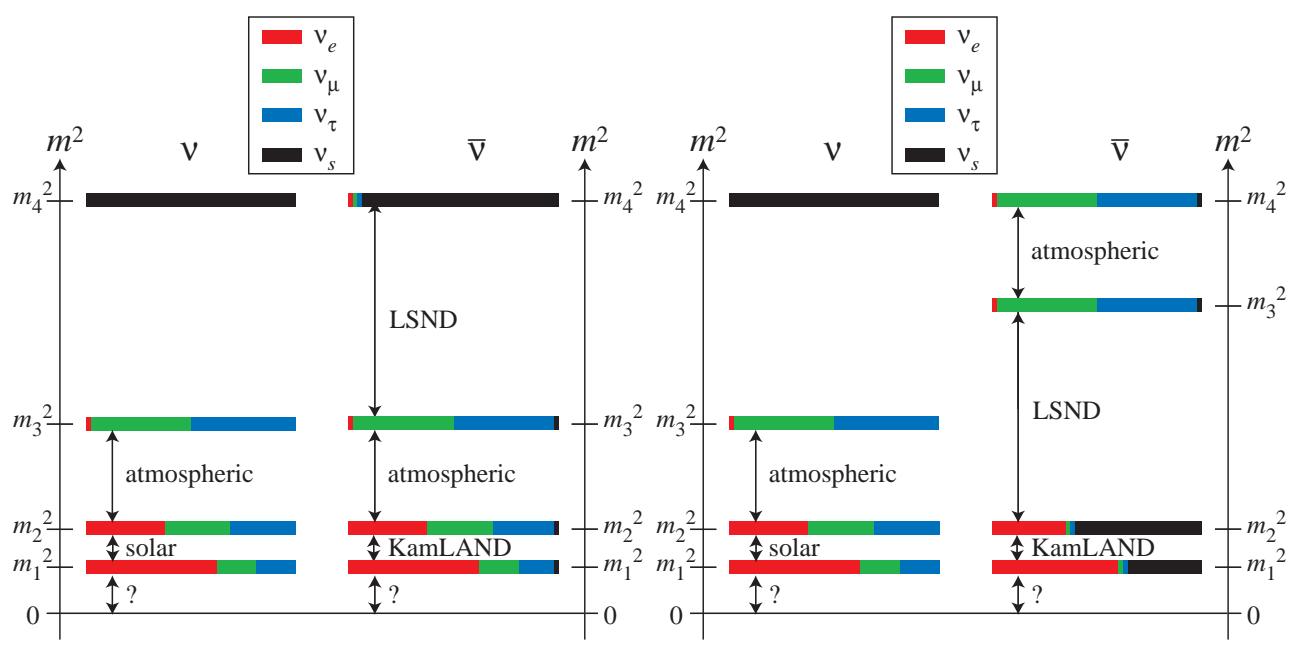

Figure 25: The revised proposal in [489] that combines CPT violation and a sterile neutrino. The neutrinos always have $2+2$ spectrum, while the anti-neutrinos may have either $3+1$ or $2+2$ spectrum.

\subsubsection{Decoherence}

So far, CPT violation as an inequality of masses between particles and antiparticles was the only way we understood CPT violation in high energy physics. However, is this CPT violation the only way a violation of this symmetry can manifest itself in nature? Such a question becomes extremely relevant for the case of LSND, because it is possible that other mechanisms leading to CPT violation exist, unrelated, in principle, to mass differences between particles and antiparticles. Such additional mechanisms for CPT violation may well be capable of explaining the LSND results within a three generation scenario without invoking a sterile neutrino (a scenario which, on the other hand, is getting totally excluded as new experimental data become available). It is therefore necessary to explore whether alternative ways exist to account for the LSND result without invoking extra (sterile) neutrino states.

Quantum decoherence is the key to answer this question. Indeed, quantum decoherence in matter propagation occurs when the matter subsystem interacts with an 'environment', according to the rules of open-system quantum mechanics. At a fundamental level, such a decoherence may be the result of propagation of matter in quantum gravity spacetime backgrounds with 'fuzzy' properties, which may be responsible for violation of CPT in a way not necessarily related to mass differences between particles and antiparticles.

A characteristic example of such a violation occurs in quantum gravity models that involve singular space-time configurations, integrated over in a path integral formalism, which are such that the axioms of quantum field theory, as well as conventional quantum mechanical behavior, cannot be maintained. Such configurations consist of wormholes, microscopic (Planck size) black holes, and other topologically non-trivial solitonic objects, such as geons etc. Collectively, we may call such configurations space time foam.

It has been argued that, as result, a mixed state description must be used ( $Q G$-induced decoherence) [495], given that such objects cannot be accessible to low-energy observers, and as such must be traced over in an effective field theory context. As a consequence of 
that CPT invariance in its strong form must be abandoned in a foamy quantum gravity theory. Such a breakdown of CPT symmetry is a fundamental one, and, in particular, implies that a proper CPT operator may be ill defined in such QG decoherence cases.

Some caution should be paid regarding CPT violation through decoherence. From a formal view point, the non-invertibility of the S-matrix, which implies a strong violation of CPT, does not preclude a softer form of CPT invariance, in the sense that any strong form of CPT violation does not necessarily have to show up in any single experimental measurement. This implies that, despite the general evolution of pure to mixed states, it may still be possible in the laboratory to ensure that the system evolves from an initial pure state to a single final state, and that the weak form of CPT invariance is manifested through the equality of probabilities between these states. If this is the case, then the decoherence-induced CPT violation will not show up in any experimental measurement.

In the parametrization of [495] for the decoherence effects, one uses three decoherence parameters with dimensions of energy, $\alpha, \beta, \gamma$, where the positivity of $\rho$, required by the fact that its diagonal elements express probability densities, implies $\alpha, \gamma \geq 0$, and $\alpha \gamma \geq \beta^{2}$. If the requirement of a completely positive map $\rho(t)$ is imposed in the two generation case, then $\mathcal{L}$ becomes diagonal, with only one non vanishing entry occupied by the decoherence parameter $\gamma>0$ [496]. Following this approach, for a three generation scenario, we will assume for the $9 \times 9$ decoherence matrix $\mathcal{L}:\left[\mathcal{L}_{\mu \nu}\right]=$ Diag $\left(0,-\gamma_{1},-\gamma_{2},-\gamma_{3},-\gamma_{4},-\gamma_{5},-\gamma_{6},-\gamma_{7},-\gamma_{8}\right)$ in direct analogy with the two-level case of complete positivity [496,497], although there is no strong physical motivation behind such restricted forms of decoherence. This assumption, however, leads to the simplest possible decoherence models, and, for our phenomenological purposes in this work, we will assume the above form, which we will use to fit all the available neutrino data. It must be clear to the reader though, that such a simplification, if proved to be successful (which, as we shall argue below, is the case here), just adds more in favor of decoherence models, given the restricted number of available parameters for the fit in this case.

In order to check these models, we have performed a $\chi^{2}$ comparison (as opposed to a $\chi^{2}$ fit) to SuperKamiokande sub-GeV and multi GeV data (40 data points), CHOOZ data (15 data points) and LSND (1 datum), for a sample point in the vast parameter space of our extremely simplified version of decoherence models. Let us emphasize that we have not performed a $\chi^{2}$-fit and therefore the point we are selecting (by "eye" and not by $\chi$ ) is not optimized to give the best fit to the existing data. Instead, it must be regarded as one among the many equally good sons in this family of solutions, being extremely possible to find a better fitting one through a complete (and highly time consuming) scan over the whole parameter space.

Cutting the long story short, and to make the analysis easier, we have set all the $\gamma_{i}$ in the neutrino sector to zero, restricting this way, all the decoherence effects to the antineutrino one where we have assumed for the sake of simplicity, $\overline{\gamma_{1}}=\overline{\gamma_{2}}=2 \cdot 10^{-18}$. $E$ and $\overline{\gamma_{3}}=\overline{\gamma_{8}}=1 \cdot 10^{-24} / E$, where $E$ is the neutrino energy, and barred quantities refer to the antineutrinos, given that decoherence takes place only in this sector in our model. All the other parameters are assumed to be zero. All in all, we have introduced only two new parameters, two new degrees of freedom, $\overline{\gamma_{1}}$ and $\overline{\gamma_{3}}$, and we shall try to explain with them all the available experimental data. (For further details we refer the reader to [493]). 
At this point it is important to stress that the inclusion of two new degrees of freedom is not sufficient to guarantee that one will indeed be able to account for all the experimental observations. We have to keep in mind that, in no-decoherence situations, the addition of a sterile neutrino (which comes along with four new degrees of freedom -excluding the possibility of CP violating phases) did not seem to be sufficient for matching all the available experimental data, at least in CPT conserving situations.

In order to test our model we have calculated the $\chi^{2}$ of the 56 data points mentioned above for different scenarios, The results with which we hope all our claims become crystal clear are summarized in the table, were we present the $\chi^{2}$ comparison for the following cases: (a) pure decoherence in the antineutrino sector, (b) pure decoherence in both sectors, (c) mixing plus decoherence in the antineutrino sector, (d) mixing plus decoherence in both sectors, and (e) mixing only - the standard scenario:

\begin{tabular}{|c|c|c|}
\hline Model & $\chi^{2}$ without LSND & $\chi^{2}$ including LSND \\
\hline \hline (a) & 980.7 & 980.8 \\
\hline (b) & 979.8 & 980.0 \\
\hline (c) & 52.2 & 52.3 \\
\hline (d) & 54.4 & 54.6 \\
\hline (e) & 53.9 & 60.7 \\
\hline
\end{tabular}

From the table it becomes clear that the mixing plus decoherence scenario in the antineutrino sector can easily account for all the available experimental information, including LSND. It is important to stress once more that our sample point was not obtained through a scan over all the parameter space, but by an educated guess, and therefore plenty of room is left for improvements. On the other hand, for the mixing-only/nodecoherence scenario, we have taken the best fit values of the state of the art analysis and therefore no significant improvements are expected. At this point a word of warning is in order: although superficially it seems that scenario (d), decoherence plus mixing in both sectors, provides an equally good fit, one should remember that including decoherence effects in the neutrino sector can have undesirable effects in solar neutrinos, especially due to the fact that decoherence effects are weighted by the distance traveled by the neutrino, something that may lead to seizable (not observed!) effects in the solar case.

One might wonder then, whether decohering effects, which affect the antineutrino sector sufficiently to account for the LSND result, have any impact on the solar-neutrino related parameters, measured through antineutrinos in the KamLAND experiment. In order to answer this question, it will be sufficient to calculate the electron survival probability for KamLAND in our model, which turns out to be $\left.P_{\bar{\nu}_{e} \rightarrow \bar{\nu}_{e}}\right|_{\text {KamLAND }} \simeq .63$, in perfect agreement with observations. It is also interesting to notice that in our model, the LSND effect is not given by the phase inside the oscillation term (which is proportional to the solar mass difference) but rather by the decoherence factor multiplying the oscillation term. Therefore the tension between LSND and KARMEN data is naturally eliminated, because the difference in length leads to an exponential suppression.

Having said that, it is now clear that decoherence models (once neutrino mixing is taken into account) are the best (and arguably the only) way to explain all the observations including the LSND result. This scenario, which makes dramatic predictions for the 
upcoming neutrino experiments, expresses a strong observable form of CPT violation in the laboratory, and in this sense, our fit gives a clear answer to the question asked in the introduction as to whether the weak form of CPT invariance is violated in Nature. It seems that, in order to account for the LSND results, we should invoke such a decoherenceinduced CPT violation, which however is independent of any mass differences between particles and antiparticles.

This CPT violating pattern, with equal mass spectra for neutrinos and antineutrinos, will have dramatic signatures in future neutrino oscillation experiments. The most striking consequence will be seen in MiniBooNE, according to our picture, MiniBooNE will be able to confirm LSND only when running in the antineutrino mode and not in the neutrino one, as decoherence effects live only in the former. Smaller but experimentally accessible signatures will be seen also in MINOS, by comparing conjugated channels (most noticeably, the muon survival probability). Higher energy neutrino beams or longbaseline experiments, will have significant deviations from the non-decoherence models, as our effects scale with energy and distance traveled, being therefore the best tool to explore decoherence models.

If the neutrino masses are actually related to decoherence as a result of quantum gravity, this may have far reaching consequences for our understanding of the Early stages of our Universe, and even the issue of Dark Energy that came up recently as a result of astrophysical observations on a current acceleration of the Universe from either distant supernovae data or measurements on Cosmic Microwave Background temperature fluctuations from the WMAP satellite. Indeed, decoherence implies an absence of a welldefined scattering S-matrix, which in turn would imply CPT violation in the strong form. A positive cosmological constant $\Lambda>0$ will also lead to an ill definition of an S-matrix, precisely due to the existence, in such a case, of an asymptotic-future de Sitter (inflationary) phase of the universe, with Hubble parameter $\sim \sqrt{\Lambda}$, implying the existence of a cosmic (Hubble) horizon. This in turn will prevent a proper definition of pure asymptotic states.

We would like to point out at this stage that the claimed value of the dark energy density component of the (four-dimensional) Universe today, $\Lambda \sim 10^{-122} M_{P}^{4}$, with $M_{P} \sim 10^{19} \mathrm{GeV}$ (the Planck mass scale), can actually be accounted for (in an amusing coincidence?) by the scale of the neutrino mass differences used in order to explain the oscillation experiments. Indeed, $\Lambda \sim\left[\left(\Delta m^{2}\right)^{2} / M_{P}^{4}\right] M_{P}^{4} \sim 10^{-122} M_{P}^{4}$ for $\Delta m^{2} \sim 10^{-5} \mathrm{eV}^{2}$, the order of magnitude of the solar neutrino mass difference assumed in oscillation experiments (which is the one that encompasses the decoherence effects). The quantum decoherence origin of this mass then would be in perfect agreement with the decoherence properties of the cosmological constant vacuum, mentioned previously. 


\section{NuTeV Physics}

The NuTeV experiment [498] at Fermilab has measured the ratios of neutral to charged current events in muon (anti)neutrino-nucleon scattering:

$$
\begin{aligned}
& R_{\nu}=\frac{\sigma\left(\nu_{\mu} N \rightarrow \nu_{\mu} X\right)}{\sigma\left(\nu_{\mu} N \rightarrow \mu^{-} X\right)}=g_{L}^{2}+r g_{R}^{2} \\
& R_{\bar{\nu}}=\frac{\sigma\left(\bar{\nu}_{\mu} N \rightarrow \bar{\nu}_{\mu} X\right)}{\sigma\left(\bar{\nu}_{\mu} N \rightarrow \mu^{+} X\right)}=g_{L}^{2}+\frac{g_{R}^{2}}{r}
\end{aligned}
$$

where

$$
r=\frac{\sigma\left(\bar{\nu}_{\mu} N \rightarrow \mu^{+} X\right)}{\sigma\left(\nu_{\mu} N \rightarrow \mu^{-} X\right)} \sim \frac{1}{2},
$$

and has determined the parameters $g_{L}^{2}$ and $g_{R}^{2}[499]$ to be

$$
\begin{aligned}
& g_{L}^{2}=0.30005 \pm 0.00137 \\
& g_{R}^{2}=0.03076 \pm 0.00110
\end{aligned}
$$

The Standard Model (SM) predictions of these parameters based on a global fit to non$\mathrm{NuTeV}$ data, cited as $\left[g_{L}^{2}\right]_{\mathrm{SM}}=0.3042$ and $\left[g_{R}^{2}\right]_{\mathrm{SM}}=0.0301$ in Ref. [498], differ from the $\mathrm{NuTeV}$ result by $3 \sigma$ in $g_{L}^{2}$. Alternatively, if the $\mathrm{SM}$ is fit to the $\mathrm{NuTeV}$ result, the preferred range of the Higgs mass is $660 \mathrm{GeV}<m_{H}$ (90\% C.L.) [500], well above the value of $m_{H} \sim 90 \mathrm{GeV}$ preferred by the non-NuTeV global fit [501].

The significance of the NuTeV result remains controversial [502], and a critical examination of the initial analysis is ongoing. Several groups are evaluating potential theoretical uncertainties arising from purely Standard Model physics which might be comparable to or larger than the quoted experimental uncertainty of the $\mathrm{NuTeV}$ result. Candidate sources of large theoretical uncertainty include next-to-leading-order (NLO) QCD corrections [503], NLO electroweak corrections [504], and parton distribution functions (especially as involves assumptions about sea-quark asymmetries) [505]. The effect of the former has been estimated to be comparable in size to the $\mathrm{NuTeV}$ experimental uncertainty, while the latter two might give rise to effects comparable in size to the full $\mathrm{NuTeV}$ discrepancy with the Standard Model. Elucidation of the actual impact of these effects on the NuTeV result awaits a reanalysis of the $\mathrm{NuTeV}$ data. However, it remains a distinct possibility that the discrepancy with the Standard Model prediction is genuine and that its resolution lies in physics beyond the Standard Model. Indeed, as Chanowitz has emphasized [500], the precision electroweak data indicate new physics whether anomalous data are excluded from global fits (since the preferred Higgs mass is then well below the direct search limit) or included in the fits (in which case anomalous data themselves demand a new physics explanation).

Note that the $\mathrm{NuTeV}$ value for $g_{L}^{2}$ in Eq. (74) is smaller than its SM prediction. This is a reflection of the fact that the ratios $R_{\nu}$ and $R_{\bar{\nu}}$ were smaller than expected by the SM. (The $g_{R}^{2}$ term is smaller than the $g_{L}^{2}$ term by an order of magnitude and is insignificant.) Thus, possible new physics explanations of the $\mathrm{NuTeV}$ anomaly would be those that suppress the neutral current cross sections over the charged current cross sections, or enhance the charged current cross sections over the neutral current cross sections. Two classes of models have been proposed which accomplish this task. 
The first class comprises models which suppress $R_{\nu}$ and $R_{\bar{\nu}}$ with the introduction of new neutrino-quark interactions, mediated by leptoquarks or extra $U(1)$ gauge bosons $\left(Z^{\prime}\right.$ 's), which interfere either destructively with the $Z$-exchange amplitude, or constructively with the $W$-exchange amplitude [502]. In order to preserve the excellent agreement between the SM and non-NuTeV data, the new interactions must selectively interfere with the $\nu_{\mu} N\left(\bar{\nu}_{\mu} N\right)$ scattering process, but little else. This severely restricts the types of interactions that may be introduced.

Ref. [502] proposes a model in which the $Z^{\prime}$ couples to $B-3 L_{\mu}$. This model must be fine-tuned to avoid $Z$ - $Z^{\prime}$ mixing [506] which would disrupt, among other things, lepton universality at the $Z$-pole. Fitting the $\mathrm{NuTeV}$ anomaly requires

$$
\frac{M_{Z^{\prime}}}{g_{Z^{\prime}}} \approx 3 \mathrm{TeV} .
$$

Bounds from direct $Z^{\prime}$ searches at the Tevatron and LEP limit the possible range of $M_{Z^{\prime}}$ to $M_{Z^{\prime}}>600 \mathrm{GeV}$ for $g_{Z^{\prime}} \sim 1$, or $2 \mathrm{GeV}<M_{Z^{\prime}}<10 \mathrm{GeV}$ for $g_{Z^{\prime}} \sim 10^{-3}$.

The $Z^{\prime}$ in the model proposed in Ref. [507] does not couple the neutrinos and quarks directly, since the gauged charge is $L_{\mu}-L_{\tau}$. Rather, it is a tunable $Z-Z^{\prime}$ mixing in the model which is responsible for suppressing the neutral channel cross section. The same mixing violates lepton universality on the $Z$-pole and prevents the mechanism from completely mitigating the $\mathrm{NuTeV}$ anomaly. $Z^{\prime}$ masses in the range $60 \mathrm{GeV}<M_{Z^{\prime}}<$ $72 \mathrm{GeV}$, or $M_{Z^{\prime}}>178 \mathrm{GeV}$ brings the theoretical value of $g_{L}^{2}$ within $1.6 \sigma$ of the NuTeV value while keeping lepton universality violation within $2 \sigma$.

In general, models in this class are constrained strongly by lepton universality, because $\nu_{\ell}$ is the $S U(2)_{L}$ partner of $\ell_{L}^{-}$. New interactions which respect the $S U(2)_{L}$ gauge symmetry cannot affect neutrino couplings alone: they necessarily affect couplings of the charged leptons. Nevertheless, they provide possible explanations of the $\mathrm{NuTeV}$ anomaly, and predict a flavor-selective gauge boson in the several $100 \mathrm{GeV}$ to $\mathrm{TeV}$ range, well within reach of the LHC.

Models of the second class suppress the $Z \nu \nu$ coupling by mixing the neutrino with heavy gauge singlet states (neutrissimos, i.e. right-handed neutrinos) [508-511]. For instance, if the $S U(2)_{L}$ active $\nu_{\mu}$ is a linear combination of two mass eigenstates with mixing angle $\theta$,

$$
\nu_{\mu}=(\cos \theta) \nu_{\text {light }}+(\sin \theta) \nu_{\text {heavy }}
$$

then the $Z \nu_{\mu} \nu_{\mu}$ coupling is suppressed by a factor of $\cos ^{2} \theta$ (assuming the heavy states are too massive to be created on-shell). Likewise, the $W \mu \nu_{\mu}$ coupling is suppressed by $\cos \theta$. Although both the numerators and denominators of $R_{\nu}$ and $R_{\bar{\nu}}$ are suppressed in such a model, the suppression of the numerators exceeds that of the denominators, and the ratios are therefore diminished. More generally, if the $Z \nu_{\ell} \nu_{\ell}$ coupling $(\ell=e, \mu, \tau)$ is suppressed by a factor of $\left(1-\varepsilon_{\ell}\right)$, then the $W \ell \nu_{\ell}$ coupling is suppressed by $\left(1-\varepsilon_{\ell} / 2\right)$, and $R_{\nu}$ and $R_{\bar{\nu}}$ are suppressed by $\left(1-\varepsilon_{\mu}\right)$.

The effect of such suppressions of the neutrino-gauge couplings is not limited to $\mathrm{NuTeV}$ observables alone. In addition to the obvious suppression of the $Z$ invisible width by a factor of $\left[1-(2 / 3)\left(\varepsilon_{e}+\varepsilon_{\mu}+\varepsilon_{\tau}\right)\right]$, all SM observables will be affected through the Fermi 
constant $G_{F}$ which is no longer equal to the muon decay constant $G_{\mu}$ :

$$
G_{F}=G_{\mu}\left(1+\frac{\varepsilon_{e}+\varepsilon_{\mu}}{2}\right)
$$

This shift in $G_{F}$ will destroy the excellent agreement between the SM and $Z$-pole observables. However, since $G_{F}$ always appears in the combination $\rho G_{F}$ in neutral current amplitudes, the agreement can be recovered by absorbing the shift in $G_{F}$ into a shift in $\rho$, or equivalently, in the oblique correction parameter $T$ [512]. Indeed, it was shown in Ref. [510], that the $Z$-pole, $\mathrm{NuTeV}$, and $W$ mass data can all be fit with the oblique correction parameters $S, T, U$, and a flavor universal suppression parameter $\varepsilon=\varepsilon_{e}=\varepsilon_{\mu}=\varepsilon_{\tau}$, the best fit values given by

$$
\begin{aligned}
S & =-0.03 \pm 0.10 \\
T & =-0.44 \pm 0.15 \\
U & =0.62 \pm 0.16 \\
\varepsilon & =0.0030 \pm 0.0010
\end{aligned}
$$

for a reference SM with $m_{H}=115 \mathrm{GeV}$. Therefore, for this class of models to work, neutrino mixing with heavy gauge singlet states must be accompanied by new physics contributions to $S, T$, and $U$. The values of $S$ and $T$ can be accommodated within the SM by simply increasing the Higgs mass to hundreds of $\mathrm{GeV}$, but the $W$ mass requires a large and positive $U$ parameter which cannot be generated within the SM. Thus, the models are not complete until some mechanism is found which explains the $W$ mass. But then, if the SM is fit to the $W$ mass alone, the preferred Higgs mass is far below direct search limits [500], which could be an indication that the $W$ mass requires new physics regardless of $\mathrm{NuTeV}$.

At first blush, the preferred value of $\varepsilon$ above is also problematic. This implies a large mixing angle, $\theta=0.055 \pm 0.010$, if interpreted as due to mixing with a single heavy state. The commonly accepted seesaw mechanism [67-70] relates the mixing angle to the ratio of the neutrino masses:

$$
\frac{m_{\text {light }}}{m_{\text {heavy }}} \approx \theta^{2}
$$

Choosing $m_{\text {light }} \sim 0.1 \mathrm{eV}$ and $m_{\text {heavy }} \sim 100 \mathrm{GeV}\left(m_{\text {heavy }}>M_{Z}\right.$ is needed to suppress $\left.\Gamma_{\text {inv }}\right)$ we find the mixing angle orders of magnitude too small: $\theta \sim 10^{-6}$. However, this result does not mean that it is impossible to have a large enough mixing angle between the light and heavy states. As pointed out in Ref. [509], in models with more than one generation, the generic mass matrix includes enough degrees of freedom to allow us to adjust all the masses and mixings independently. Concrete examples of models with large mass hierarchies AND large mixing angles can be found in Refs. [511,513]. What is sacrificed, however, is the traditional seesaw explanation of the small neutrino mass: i.e. since the Majorana mass $M$ in the neutrino mass matrix should be of the order of the GUT scale, the neutrino mass $m_{\text {light }} \sim m^{2} / M$ is naturally suppressed if the Dirac mass $m$ is comparable to that of the other fermions. An alternative mechanism is used in Ref. [511]. There, an intergenerational symmetry is imposed on the neutrino mass texture which reduces its rank, generating naturally light (massless) mass eigenstates. 
Abandoning the seesaw mechanism also frees the masses of the heavy states from being fixed at the GUT scale. Indeed, in the model discussed in Ref. [511], the assumption that neutrinos and up-type quarks have a common Dirac mass implies that the masses of the heavy state could be a few $\mathrm{TeV}$, well within the reach of the LHC. Without quark-lepton unification $m_{\text {heavy }}$ could be even lighter, rendering them accessible to Tevatron Run II.

Because of the large mixing angles between the light and heavy states in this class of models, flavor changing processes mediated by the heavy states may be greatly enhanced $[40,511,513,514]$. As a result, stringent constraints can be placed on the models from the experimental limits on $\mu \rightarrow e \gamma, \tau \rightarrow \mu \gamma$, [145] $\mu$-e conversion in nuclei [515,516], muoniumantimuonium oscillation $[517,518]$, etc. For instance, the MEGA limit on $\mu \rightarrow e \gamma$ leads to the constraint [511]

$$
\varepsilon_{e} \varepsilon_{\mu} \approx 0
$$

Therefore, lepton universality among the $\varepsilon_{\ell}$ must be broken maximally. Ref. [519] shows that it is possible to fit the $Z$-pole, $\mathrm{NuTeV}$, and lepton universality data while satisfying this condition.

The MEG (Mu-E-Gamma) experiment at PSI [520] plans to improve upon the MEGA limit by about two orders of magnitude. The MECO (Muon on Electron COnversion) experiment at Brookhaven [521] aims to improve the limits on $\mu-e$ conversion in nuclei by three orders of magnitude. Further constraints can be obtained from muon $g-2[522,523]$, and the violation of CKM unitarity [524-526].

The $\mathrm{NuTeV}$ anomaly, even if it does not ultimately endure sustained scrutiny, stirs us to look past orthodoxies in our model-building (seesaw, SUSY, GUTs,...) and to ask broadly what is permitted by the data. The neutrino mixing solution is relatively conservative in its use of the neutrino sector to address the $\mathrm{NuTeV}$ question. Nonetheless, it makes interesting predictions about new particles at LHC, can be probed by a wide range of neutrino oscillation experiments, precision measurements and rare decay searches, and introduces an alternative to the seesaw paradigm. Whether this or another solution resolves the $\mathrm{NuTeV}$ anomaly, the $\mathrm{NuTeV}$ result serves to focus the imagination of the theorist on the opportunities presented by the experiments. 


\section{Conclusions}

In this report, we have presented a brief review of the present ${ }^{14}$ knowledge of neutrino physics and what we can learn from the planned experiments in the next decade. Three very important measurements that are guaranteed to have a significant impact on the search for physics beyond the Standard Model are: (i) the rate of $\beta \beta_{0 \nu}$, which will inform us not only whether the neutrino is a Majorana or Dirac particle but may also provide information about the neutrino masses; (ii) the value of $\theta_{13}$, which will considerably narrow the field of flavor models and (iii) the sign of the $\Delta m_{13}^{2}$, which determines the neutrino mass hierarchy and will also help guide our understanding of flavor physics. Within the three neutrino picture, more precise measurements of the solar and atmospheric mixing angles will be helpful in discriminating among various new physics possibilities.

Important though somewhat model-dependent constraints can be drawn from experimental searches for charged lepton flavor violating processes, such as $\mu \rightarrow e \gamma$ or $\mu \rightarrow e$ conversion in nuclei, and from searches for nonzero electric dipole moments of leptons. Keep in mind that the matter-antimatter symmetry of the Universe may have its explanation in the very same mechanism that generates the small neutrino masses, and that we may be able to test this hypothesis with enough low-energy information.

Beyond the three neutrino picture, a very important issue is the status of the LSND result and whether the existence of light sterile neutrinos can be inferred from terrestrial neutrino oscillations experiments. The results of MiniBooNE, assuming they confirms those from LSND, have the potential to revolutionize our current understanding of neutrinos. Even if MiniBooNE does not confirm the LSND result, sterile neutrino effects can still be present in different channels at a more subdominant level, as has been suggested in several theoretical models.

Another important issue in neutrino physics is the magnetic moment of the neutrino, which is expected at to be nonzero but very small within the standard picture of $\mathrm{eV}$ sized neutrino masses and in the absence of new physics at the $\mathrm{TeV}$ scale. Thus, evidence for a nonzero neutrino magnetic moment close to the current astrophysical limit of $10^{-11} \mu_{B}$ would have to be interpreted as evidence of $\mathrm{TeV}$ scale new physics such as $\mathrm{TeV}$ scale leftright models, horizontal models, or large extra dimensions. Other unique probes of $\mathrm{TeV}$ scale physics are provided by neutrino oscillation experiments, thanks to their sensitivity to non-standard neutrino interactions.

Finally, one can use results in neutrino physics to test the limits of the assumptions on which the Standard Model is based, including Lorentz and CPT invariance.

\section{Acknowledgments}

The work of R.N.M. is supported by the National Science Foundation grant No. PHY0099544 and PHY-0354401. The work of W.R. and M.L. is supported by the "Deutsche Forschungsgemeinschaft" in the "Sonderforschungsbereich 375 für Astroteilchenphysik" and under project number RO-2516/3-1 (W.R.). The work of M.R. is partially supported by the EU 6th Framework Program MRTN-CT-2004-503369 "Quest for Unification" and

\footnotetext{
${ }^{14}$ As of the end of summer, 2005.
} 
MRTN-CT-2004-005104 "ForcesUniverse". The work of R.S. is supported in part by the NSF grant NSF-PHY-00-98527. The work of J.K. is supported by the "Impuls- und Vernetzungsfonds" of the Helmholtz Assciation, contract number VH-NG-006. The work of S.A. is supported by the PPARC grant PPA/G/O/2002/00468. The work of A.d.G. is sponsored in part by the US Department of Energy Contract DE-FG02-91ER40684. We thank F. Vissani for participating in the shorter version of this report. 


\section{References}

[1] R.N. Mohapatra et al., hep-ph/0412099.

[2] B.T. Cleveland et al., Astrophys. J. 496 (1998) 505; Y. Fukuda et al., Phys. Rev. Lett. 77 (1996) 1683; V. Gavrin, Nucl. Phys. Proc. Suppl. 91 (2001) 36; W. Hampel et al., Phys. Lett. B447 (1999) 127; M. Altmann et al., Phys. Lett. B490 (2000) 16; Super-Kamiokande Coll., Y. Fukuda et al., Phys. Rev. Lett. 86 (2001) 5651; Q. R. Ahmad et al. [SNO Collaboration], Phys. Rev. Lett. 87 (2001) 071301 nucl-ex/0106015; Q. R. Ahmad et al. [SNO Collaboration], Phys. Rev. Lett. 89 (2002) 011301 nucl-ex/0204008]; S. N. Ahmed et al. [SNO Collaboration], Phys. Rev. Lett. 92, 181301 (2004) nucl-ex/0309004; K. Eguchi et al. [KamLAND Collaboration], Phys. Rev. Lett. 90 (2003) 021802 hep-ex/0212021; Y. Fukuda et al. [Super-Kamiokande Collaboration], Phys. Rev. Lett. 81, 1562 (1998) hep-ex/9807003. W. Allison et al., Phys. Lett. B391, 491 (1997); Phys. Lett. B449, 137 (1999). Reviews of solar neutrinos include J. Bahcall's URL http://www.ias.edu jnb and a review of atmospheric neutrino data is C. K. Jung, C. McGrew, T. Kajita, and T. Mann, Ann. Rev. Nucl. Part. Sci. 51, 451 (2001); see also the web site http://neutrinooscillation.org.

[3] Current experimental results are compiled in the Particle Data Group, Review of Particle Physics, at http://pdg.lbl.gov.

[4] R. N. Mohapatra and P. B. Pal, Massive Neutrinos in Physics and Astrophysics, 3rd ed. (World Scientific, Singapore, 2004); V. Barger, K. Whisnant and D. Marfatia, Int. J. Mod. Phys. E12, 569 (2003); C. Gonzales-Garcia and Y. Nir, Rev. Mod. Phys. 75, 345 (2003); A. Smirnov, Int. J. Mod. Phys. A 19, 1180 (2004) hep-ph/0311259; S. Pakvasa and J. W. F. Valle, hep-ph/0301061; S. T. Petcov, hep-ph/0412410; M. Fukugita and T. Yanagida, Physics of Neutrinos and Applications in Astrophysics (Springer, Berlin, 2003). For some earlier reviews, see S. M. Bilenky, C. Giunti and W. Grimus, Prog. Part. Nucl. Phys. 43 (1999) 1; F. Boehm and P. Vogel, Physics of Massive Neutrinos (2nd ed., Cambridge Univ. Press, Cambridge, 1992); C. W. Kim and A. Pevsner, Neutrinos in Physics and Astrophysics (Harwood, Reading, 1992); S. M. Bilenky and S. T. Petcov, Rev. Mod. Phys. 59, 67 (1987). See also a recent focus issue on Neutrinos by "New Journal of Physics" edited by F. Halzen, M. Lindner and A. Suzuki; there is an extensive web site that not only reviews the history of the early developments in the field but also provides a very up-to-date list of references of the important papers maintained by C. Giunti and Marco Leveder; entitled "Neutrino Unbound" at http://www.nu.to.infn.it/

[5] H. Back et al., hep-ex/0412016

[6] E. Anderson et al., available online at http://www.aps.org/neutrino/index.cfm Part of the APS Neutrino Study.

[7] C. Albrightet al., available online at http://www.aps.org/neutrino/index.cfm

[8] C. Aalseth et al., hep-ph/0412300. 
[9] S.W. Barwick et al., astro-ph/0412544.

[10] B. Pontecorvo, Zh. Eksp. Teor. Fiz. 33 (1957) 549 and 34 (1958) 247; Z. Maki, M. Nakagawa and S. Sakata, Prog. Theor. Phys. 28 (1962) 870 (discussed the $2 \times 2$ case); B. Pontecorvo, Zh. Eksp. Teor. Fiz. 53 (1967) 1717; V. N. Gribov and B. Pontecorvo, Phys. Lett. B 28, 493 (1969).

[11] S. M. Bilenky, J. Hosek and S. T. Petcov, Phys. Lett. B94 (1980) 495;

[12] J. Schechter and J.W.F. Valle, Phys. Rev. D22 (1980) 2227; M. Doi et al., Phys. Lett. B102 (1981) 323.

[13] For recent analyses, see J. N. Bahcall, M. C. Gonzalez-Garcia and C. Pena-Garay, JHEP 0408, 016 (2004) [arXiv;hep-ph/0406294]; C. Gonzalez-Garcia and M. Maltoni, hep-ph/0406056; M. Maltoni, T. Schwetz, M. A. Tortola and J. W. F. Valle, hep-ph/0405172; A. Bandyopadhyay et al., hep-ph/0406328; S. Goswami, A. Bandyopadhyay and S. Choubey, hep-ph/0409224; G. Fogli, E. Lisi, in Altarelli, G. (ed.) et al.: Neutrino mass, 2003, pp. 135.

[14] M. Apollonio et al., Phys. Lett. B466 (1999) 415; F. Boehm et al., Phys. Rev. D62 (2000) 072002.

[15] L. Wolfenstein, Phys. Rev.D 17, 2369 (1978); S. P. Mikheyev and A. Smirnov, Nuovo Cimento C 9, 17 (1986).

[16] V. Barger, R. Phillips and K. Whisnant, Phys. Rev. D 34, 980 (1986); H. Bethe, Phys. Rev. Lett. 56, 1305 (1986); W. C. Haxton, Phys. Rev. Lett. 57, 1271 (1986); S. Parke, Phys. Rev. Lett. 57, 1275 (1986); S. P. Rosen and J. Gelb, Phys. Rev. D34, 969 (1986); T. K. Kuo and J. Pantaleone, Phys. Rev. Lett. 57, 1805 (1986); P. Langacker et al., Nucl. Phys. B 282 (1987) 589; S.T. Petcov, Phys. Lett. B200 (1988) 373; A. Friedland, Phys. Rev. D64 (2001) 013008; E. Lisi et al., Phys. Rev. D 63 (2001) 093002.

[17] J. N. Bahcall, M. C. Gonzalez-Garcia and C. Pena-Garay, JHEP 0408, 016 (2004) [arXiv:hep-ph/0406294.

[18] G. M. Fuller, J. R. Primack and Y. Z. Qian, Phys. Rev. D 52, 1288 (1995) [arXiv:astro-ph/9502081; D. O. Caldwell and R. N. Mohapatra, Phys. Lett. B 354, 371 (1995) [arXiv:hep-ph/9503316; G. Raffelt and J. Silk, Phys. Lett. B 366, 429 (1996) [arXiv:hep-ph/9502306.

[19] D. Caldwell and R. N. Mohapatra, Phys. Rev. D 48 , 3259 (1993); A. Joshipura, Phys. Rev. D51, 1321 (1995); K. S. Babu, E. Ma and J. W. F. Valle, hep-ph/0206292 S. Antusch and S. F. King, Nucl. Phys. B 705 (2005) 239, hep-ph/0402121.

[20] C. Kraus et al., Eur. Phys. J. C 40, 447 (2005) [arXiv:hep-ex/0412056.

[21] V. M. Lobashev et al., Phys. Lett. B 460, 227 (1999). 
[22] A. Osipowicz et al. [KATRIN Collaboration], hep-ex/0109033.

[23] S.M. Bilenky and S.T. Petcov in [4].

[24] S. R. Elliott and P. Vogel, Ann. Rev. Nucl. Part. Sci. 52 (2002) 115; A. Morales and J. Morales, Nucl. Phys. Proc. Suppl. 114, 141 (2003) hep-ph/0211332].

[25] R. N. Mohapatra and J. Vergados, Phys. Rev. Lett. 47, 1713 (1981); R. N. Mohapatra, Phys. Rev. D 34, 3457 (1986); B. Brahmachari and E. Ma, Phys. Lett. B536, 259 (2002).

[26] J. Schechter, J. W. F. Valle, Phys. Rev. D25 (1982) 2951; E. Takasugi, Phys. Lett. B149 (1984) 372.

[27] H. V. Klapdor-Kleingrothaus et al., Eur. Phys. J. A 12, 147 (2001) hep-ph/0103062.

[28] C. E. Aalseth et al. [16EX Collaboration], Phys. Rev. D 65, 092007 (2002) hep-ex/0202026.

[29] G. L. Fogli et al., Phys. Rev. D 70, 113003 (2004).

[30] H. V. Klapdor-Kleingrothaus, I. V. Krivosheina, A. Dietz and O. Chkvorets, Phys. Lett. B 586, 198 (2004) hep-ph/0404088.

[31] H. V. Klapdor-Kleingrothaus, A. Dietz, H. L. Harney and I. V. Krivosheina, Mod. Phys. Lett. A16 (2001) 2409-2420 hep-ph/0201231.

[32] C. E. Aalseth et al., Mod. Phys. Lett. A 17, 1475 (2002) hep-ex/0202018; H. V. Klapdor-Kleingrothaus, hep-ph/0205228; H. L. Harney, hep-ph/0205293;

[33] C. L. Bennett et al., Astrophys. J. Suppl. 148, 1 (2003) astro-ph/0302207.

[34] S. Hannestad, hep-ph/0310220.

[35] A. D. Dolgov, K. Kainulainen and I. Z. Rothstein, Phys. Rev. D 51, 4129 (1995) hep-ph/9407395.

[36] B. Kayser, in CP violation, ed. C. Jarlskog (World Scientific, 1988); Z.-z. Xing, Int. J. Mod. Phys. A 19, 1 (2004) hep-ph/0307359.

[37] A. de Gouvêa, B. Kayser and R. N. Mohapatra, Phys.Rev. D67, 053004 (2003).

[38] H. Minakata, H. Nunokawa and S. Parke, Phys. Rev. D66, 093012 (2002) hep-ph/0208163; J. Burguet-Castell, M. B. Gavela, J. J. Gomez-Cadenas, P. Hernandez and O. Mena, Nucl. Phys. B646 (2002) 301 (2002); H. Minakata, hep-ph/0402197 and references therein.

[39] S.M. Bilenky et al., Phys. Rev. D56 (1996) 4432.

[40] B. W. Lee and R. E. Shrock, Phys. Rev. D16, 1444 (1977). 
[41] J. Schechter and J. W. F. Valle, Phys. Rev. D 22, 2227 (1980).

[42] D. Caldwell and R. N. Mohapatra, Phys. Rev. D 46, 3259 (1993); J. Peltoniemi and J. W. F. Valle, Nucl. Phys. B 406, 409 (1993); J. Peltoniemi, D. Tommasini and J. W. F. Valle, Phys. Lett. B 298, 383 (1993).

[43] LSND collaboration, Phys. Rev. Lett. 77, 3082 (1996).

[44] B. Armbruster et al. Phys. Rev. D65, 112001 (2002).

[45] A. O. Bazarko, [BooNE collaboration], hep-ex/9906003.

[46] S. Bilenky, W. Grimus, C. Giunti and T. Schwetz, hep-ph/9904316, V. Barger, B. Kayser, J. Learned, T. Weiler and K. Whisnant, Phys. Lett. B 489, 345 (2000); for a review, see S. Bilenky, C. Giunti and W. Grimus, Prog. Part. Nucl. Phys. 43, 1 (1999).

[47] M. Sorel, J. Conrad and M. Shavitz, hep-ph/0305255.

[48] R. Shrock, Phys. Rev. D 9, 743 (1974).

[49] J. Kim, Phys. Rev. D 14, 3000 (1976).

[50] W. Marciano and A. I. Sanda, Phys. Lett. B 67, 303 (1977).

[51] M. Beg, W. Marciano, and M. Ruderman, Phys. Rev. D 17, 1395 (1978).

[52] K. Fujikawa and R. E. Shrock, Phys. Rev. Lett. 45, 963 (1980).

[53] R. E. Shrock, Nucl. Phys. B206, 359 (1982).

[54] P. Pal and L. Wolfenstein, Phys. Rev. D25, 766 (1982).

[55] B. Kayser, Phys. Rev. D26, 1662 (1982).

[56] C. S. Lim and W. Marciano, Phys. Rev. D37, 1368 (1988).

[57] R. Barbieri and R. N. Mohapatra, Phys. Lett. B218, 225 (1989).

[58] K.S. Babu and R.N. Mohapatra, Phys. Rev. Lett. 63, 228 (1989); Phys. Rev. Lett. 64, 1705 (1990).

[59] R. N. Mohapatra, S.-P. Ng, and H.-B. Yu, Phys. Rev. D70, 057301 (2004).

[60] See G. Raffelt, Stars as Laboratories for Fundamental Physics, Chicago University Press (1996).

[61] S. T. Petcov, Sov. J. Nucl. Phys. 25 (1977) 340; (E) 25 (1977) 698.

[62] F. Wilczek and A. Zee, Phys. Rev. Lett. 38 (1977) 531.

[63] Y. Chikashige, R. N. Mohapatra and R. D. Peccei, Phys. Lett. B 98, 265 (1981). 
[64] J. Schechter and J. W. F. Valle, Phys. Rev. D 25, 774 (1982); J. W. F. Valle, Phys. Lett. B 131, 87 (1983);G. B. Gelmini and J. W. F. Valle, Phys. Lett. B 142, 181 (1984).

[65] J. F. Beacom, N. F. Bell, D. Hooper, S. Pakvasa and T. J. Weiler, Phys. Rev. D 69, 017303 (2004) hep-ph/0309267.

[66] P. Minkowski, Phys. Lett. B67 , 421 (1977).

[67] T. Yanagida, in Proceedings of the Workshop on the Unified Theory and the Baryon Number in the Universe (O. Sawada and A. Sugamoto, eds.), KEK, Tsukuba, Japan, 1979 , p. 95.

[68] M. Gell-Mann, P. Ramond, and R. Slansky, Complex spinors and unified theories, in Supergravity (P. van Nieuwenhuizen and D. Z. Freedman, eds.), North Holland, Amsterdam, 1979, p. 315.

[69] S. L. Glashow, The future of elementary particle physics, in Proceedings of the 1979 Cargèse Summer Institute on Quarks and Leptons (M. Lévy, J.-L. Basdevant, D. Speiser, J. Weyers, R. Gastmans, and M. Jacob, eds.), Plenum Press, New York, 1980, pp. 687-713.

[70] R. N. Mohapatra and G. Senjanović, Phys. Rev. Lett. 44 (1980), 912.

[71] A. de Gouvêa, hep-ph/0501039.

[72] J. C. Pati and A. Salam, Phys. Rev. D10, 275 (1974); R. N. Mohapatra and J. C. Pati, Phys. Rev. D 11, 566, 2558 (1975); G. Senjanović and R. N. Mohapatra, Phys. Rev. D 12, 1502 (1975).

[73] G. Lazarides, Q. Shafi and C. Wetterich, Nucl.Phys.B181, 287 (1981); R. N. Mohapatra and G. Senjanović, Phys. Rev. D 23, 165 (1981).

[74] R. N. Mohapatra and R. E. Marshak, Proceedings of the Orbis Scientiae, January, 1980, p. 277 (Plenum Press, ed. B. Korsonoglu et al); J. Schechter and J. W. F. Valle, Phys. Rev. D 22, 2227 (1980). T. P. Cheng and L. F. Li, Phys. Rev. D 22, 2860 (1980).

[75] R. N. Mohapatra and P. B. Pal, Massive neutrinos in physics and astrophysics, vol. 1, 1990, p. 127 and 128; Eq. 7.19; E. Ma and U. Sarkar, Phys. Rev. Lett. 80, 5716 (1998).

[76] E. Ma, Phys. Rev. Lett.81, 1171 (1998).

[77] For recent reviews, see S. F. King, Rept. Prog. Phys. 67, 107 (2004); G. Altarelli and F. Feruglio, hep-ph/0405048; New J.Phys. 6 , 106 (2004). 
[78] M. Raidal, hep-ph/0404046 H. Minakata and A. Y. Smirnov, hep-ph/0405088; P. H. Frampton and R. N. Mohapatra, hep-ph/0407139; W. Rodejohann, Phys. Rev. D 69, 033005 (2004); J. Ferrandis and S. Pakvasa, hep-ph/0412038 S. Antusch, S. F. King and R. N. Mohapatra, arXiv hep-ph/0504007; S. K. Kang, C. S. Kim and J. Lee, hep-ph/0501029 N. Li and B. Q. Ma, hep-ph/0501226; K. Cheung, S. K. Kang, C. S. Kim and J. Lee, hep-ph/0503122; T. Ohlsson, hep-ph/0506094.

[79] Leptonic symmetries $L_{e}-L_{\mu} \pm L_{\tau}$ and $L_{e} \pm L_{\mu}-L_{\tau}$ of neutrino masses were discussed early on in S. T. Petcov, Phys. Lett. B 110, 245 (1982); The specific combination $L_{e}-L_{\mu}-L_{\tau}$ were discussed in R. Barbieri, L. Hall, D. Smith, A. Strumia and N. Weiner, JHEP 12, 017 (1998); A. Joshipura and S. Rindani, Eur. Phys. J. C14, 85 (2000); R. N. Mohapatra, A. Perez-Lorenzana, C. A. de S. Pires, Phys. Lett. B474, 355 (2000); T. Kitabayashi and M. Yasue, Phys. Rev. D63, 095002 (2001); Phys. Lett. B508, 85 (2001); Phys. Lett. B524, 308 (2002) hep-ph/0110303; L. Lavoura, Phys. Rev. D62, 093011 (2000); W. Grimus and L. Lavoura, Phys. Rev. D62, 093012 (2000); J. High Energy Phys. 09, 007 (2000); J. High Energy Phys. 07, 045 (2001); R. N. Mohapatra, Phys. Rev. D64, 091301 (2001) hep-ph/0107264|; K. S. Babu and R. N. Mohapatra, Phys. Lett. B532, 77 (2002); H. S. Goh, R. N. Mohapatra and S. P. Ng, Phys. Lett. B542, 116 (2002) hep-ph/0205131|; D. A. Dicus, H.-J. He, J. N. Ng, Phys. Lett. B536, 83 (2002); Q. Shafi and Z. Tavartkiladze, Phys. Lett. B482, 1451 (2000); S. T. Petcov and W. Rodejohann, hep-ph/0409135.

[80] K. S. Babu, C. N. Leung, and J. Pantaleone, Phys. Lett. B319 (1993), 191-198 hep-ph/9309223.

[81] K. R. S. Balaji, A. S. Dighe, R. N. Mohapatra, and M. K. Parida, Phys. Rev. Lett. 84 (2000), 5034-5037 hep-ph/0001310.

[82] S. Antusch and M. Ratz, JHEP 0211, 010 (2002) [arXiv:hep-ph/0208136].

[83] R. N. Mohapatra, M. K. Parida and G. Rajasekaran, Phys. Rev. D 69, 053007 (2004) hep-ph/0301234.

[84] C. Hagedorn, J. Kersten and M. Lindner, Phys. Lett. B 597, 63 (2004) [arXiv hep-ph/0406103.

[85] C. H. Albright, K. S. Babu and S. M. Barr, Phys. Rev. Lett. 81, 1167 (1998) hep-ph/9802314.

[86] A. Zee, Phys. Lett. B93, 389 (1980).

[87] T. P. Cheng and L. F. Li, Phys. Rev. D 22, 2860 (1980).

[88] K. S. Babu, Phys. Lett. B 203, 132 (1988).

[89] D. Chang and R. N. Mohapatra, Phys. Rev. Lett. 58,1600 (1987)

[90] R. N. Mohapatra, Phys. Rev. Lett. 56, 561 (1986). R. N. Mohapatra and J. W. F. Valle, Phys. Rev. D 34, 1642 (1986); E. Witten, Nucl. Phys. B 268, 79 (1986). 
[91] J. Gluza, Acta Phys. Polon. B33 (2002) 1735.

[92] T. Appelquist and R. Shrock, Phys. Lett. B 548, 204 (2002).

[93] T. Appelquist and R. Shrock, Phys. Rev. Lett. 90, 201801 (2003).

[94] Z. Chacko, L. Hall, S. Oliver and M. Perelstein, hep-ph/0405067.

[95] P. Langacker, Phys. Rev. D 58, 093017 (1998).

[96] A. Ilakovac and A. Pilaftsis, Nucl. Phys. B 437, 491 (1995).

[97] G. Barenboim, G. C. Branco, A. de Gouvêa and M. N. Rebelo, Phys. Rev. D 64, 073005 (2001).

[98] see, for example, M. Frigerio and A.Yu. Smirnov, Nucl. Phys. B 640, 233 (2002); Phys. Rev. D 67, 013007 (2003).

[99] V. Barger, S.L. Glashow, P. Langacker and D. Marfatia, Phys. Lett. B 540, 247 (2002).

[100] S. Pascoli, S. T. Petcov and W. Rodejohann, Phys. Lett. B 549, 177 (2002).

[101] A. Broncano, M. B. Gavela, E. Jenkins, Nucl. Phys. B672, 163 (2003).

[102] P. H. Frampton, S. T. Petcov and W. Rodejohann, Nucl. Phys. B 687, 31 (2004) hep-ph/0401206.

[103] for recent studies see, for example, G. Altarelli, F. Feruglio and I. Masina, Nucl. Phys. B 689, 157 (2004); A. Romanino, hep-ph/0402258; and references therein.

[104] S. Antusch and S. F. King, Phys. Lett. B 591 (2004) 104, hep-ph/0403053.

[105] L.J. Hall, H. Murayama and N. Weiner, Phys. Rev. Lett. 84, 2572 (2000); N. Haba and H. Murayama, Phys. Rev. D 63, 053010 (2001).

[106] A. de Gouvêa and H. Murayama, Phys. Lett. B 573, 94 (2003).

[107] A. de Gouvêa, Phys. Rev. D 69, 093007 (2004) [arXiv:hep-ph/0401220].

[108] F. Vissani, hep-ph/9708483 V. Barger, S. Pakvasa, T. Weiler and K. Whisnant, Phys. Lett. B 437, 107 (1998); A. Baltz, A.S. Goldhaber and M. Goldhaber, Phys. Rev. Lett. 815730 (1998); G. Altarelli and F. Feruglio, Phys. Lett. B 439, 112 (1998); M. Jezabek and Y. Sumino, Phys. Lett. B 440, 327 (1998); D. V. Ahluwalia, Mod. Phys. Lett. A13, 2249 (1998).

[109] S. Lavignac, I. Masina and C.A. Savoy, Nucl. Phys. B 633 (2002) 139, hep-ph/0202086. 
[110] T. Fukuyama and H. Nishiura, hep-ph/9702253; R. N. Mohapatra and S. Nussinov, Phys. Rev. D60 (1999) 013002; E. Ma and M. raidal, Phys. Rev. Lett. 87 (2001) 011802; C. S. Lam, hep-ph/0104116; T. Kitabayashi and M. Yasue, Phys.Rev. D67 015006 (2003); W. Grimus and L. Lavoura, hep-ph/0305046; 0309050; Y. Koide, Phys.Rev. D69 (2004) 093001; A. Ghosa, Mod. Phys. Lett. A 19 (2004) 2579.

[111] R. N. Mohapatra, Slac Summer Inst. lecture; http://www-conf.slac.stanford.edu/ssi/2004 hep-ph/0408187 JHEP, 10, 027 (2004); W. Grimus, A. S.Joshipura, S. Kaneko, L. Lavoura, H. Sawanaka, M. Tanimoto, hep-ph/0408123; R. N. Mohapatra and W. Rodejohann, Phys. Rev. D 72, 053001 (2005) [arXiv hep-ph/0507312].

[112] P. F. Harrison, D. H. Perkins and W. G. Scott, Phys. Lett. B 458, 79 (1999); Phys. Lett. B 530, 167 (2002); P. F. Harrison and W. G. Scott, Phys. Lett. B 535, 163 (2002); Phys. Lett. B 557, 76 (2003); Z. Z. Xing, Phys. Lett. B 533, 85 (2002). X. G. He and A. Zee, Phys. Lett. B 560, 87 (2003); E. Ma, Phys. Rev. Lett. 90, 221802 (2003); Phys. Lett. B 583, 157 (2004); C. I. Low and R. R. Volkas, Phys. Rev. D 68, 033007 (2003); S. H. Chang, S. K. Kang and K. Siyeon, Phys. Lett. B 597, 78 (2004); E. Ma, Phys. Rev. D 70, 031901 (2004); F. Plentinger and W. Rodejohann, Phys. Lett. B 625, 264 (2005); P. F. Harrison and W. G. Scott, hep-ph/0508012; S. Luo and Z. Z. Xing, hep-ph/0509065 W. Grimus and L. Lavoura, hep-ph/0509239; Originally, a very similar, but with recent data incompatible form of $U$ has been proposed already in L. Wolfenstein, Phys. Rev. D 18, 958 (1978).

[113] G. Altarelli, F. Ferruglio, hep-ph/0504165 E. Ma, hep-ph/0505209 S. F. King, JHEP 0508, 105 (2005); I. de Medeiros Varzielas and G. G. Ross, hep-ph/0507176; K. S. Babu and X. G. He, hep-ph/0507217.

[114] P. Langacker et al., Nucl. Phys. B 282 (1987) 589.

[115] W. Rodejohann, J. Phys. G 28, 1477 (2002).

[116] I-H. Lee, Phys. Lett. B 138, 121 (1984); Nucl. Phys. B 246, 120 (1984).

[117] J. Ellis and M. Raidal, Nucl. Phys. B643 (2002) 229 hep-ph/0206174.

[118] S. Pascoli, S.T. Petcov and C.E. Yaguna, Phys. Lett. B564 (2003) 241 hep-ph/0301095.

[119] S.M. Bilenky, S. Pascoli and S.T. Petcov, Phys. Rev. D64 (2001) 053010.

[120] S.T. Petcov and A.Yu. Smirnov, Phys. Lett. B322 (1994) 109.

[121] F. Feruglio, A. Strumia and F. Vissani, Nucl. Phys. B 637 (2002) 345 [Addendumibid. B 659 (2003) 359].

[122] S. Pascoli and S.T. Petcov, hep-ph/0308034 
[123] S.M. Bilenky et al., Phys. Lett. B465 (1999) 193.

[124] V. Barger and K. Whisnant, Phys. Lett. B456 (1999) 194; H. Minakata and O. Yasuda, Nucl. Phys. B523 (1998) 597; T. Fukuyama et al., Phys. Rev. D57 (1998) 5844 and Mod. Phys. Lett. A17 (2002) 2597; P. Osland and G. Vigdel, Phys. Lett. B520 (2001) 128; D. Falcone and F. Tramontano, Phys. Rev. D64 (2001) 077302; T. Hambye, Eur. Phys. J. direct C4 (2002) 13;

[125] F. Vissani, JHEP 06 (1999) 022; M. Czakon et al., Phys. Lett. B465 (1999) 211, hep-ph/0003161 and Phys. Rev. D65 (2002) 053008; H. V. Klapdor-Kleingrothaus, H. Pas and A. Yu. Smirnov, Phys. Rev. D63 (2001) 073005; H. Minakata and H. Sugiyama, Phys. Lett. B526 (2002) 335; N. Haba, N. Nakamura and T. Suzuki, hep-ph/0205141.

[126] W. Rodejohann, Nucl. Phys. B 597 (2001) 110, and hep-ph/0203214.

[127] S. Pascoli and S.T. Petcov, Phys. Lett. B 580 (2004) 280.

[128] A. Bandyopadhyay et al., Phys. Lett. B 583 (2004) 134.

[129] G.L. Fogli et al., Phys. Rev. D67 (2003) 093006.

[130] G. L. Fogli, E. Lisi, A. Marrone, D. Montanino, A. Palazzo and A. M. Rotunno, Phys. Rev. D 69, 017301 (2004) hep-ph/0308055.

[131] S. Pascoli and S.T. Petcov, Phys. Lett. B544 (2002) 239.

[132] A. Bandyopadhyay et al., hep-ph/0406328.

[133] H. Murayama and C. Peña-Garay, Phys. Rev. D69 (2004) 031301.

[134] S. Pascoli, S.T. Petcov and L. Wolfenstein, Phys. Lett. B524 (2002) 319; S. Pascoli and S. T. Petcov, Phys. Atom. Nucl. 66, 444 (2003) [Yad. Fiz. 66, 472 (2003)] hep-ph/0111203.

[135] V. A. Rodin et al., Phys. Rev. C68 (2003) 044302.

[136] S. Pascoli, S.T. Petcov and W. Rodejohann, Phys. Lett. B558 (2003) 141.

[137] K.S. Babu and R. N. Mohapatra, Phys. Rev. Lett. 75 (1995) 2276; M. Hirsch, H.V. Klapdor-Kleingrothaus and S.G. Kovalenko Phys. Lett. B398 (1977) 311; ibid. B459 (1999) 450.

[138] S. Pascoli, S. T. Petcov and W. Rodejohann, Phys. Rev. D 68, 093007 (2003) hep-ph/0302054.

[139] S.F. King, JHEP 0209 (2002) 011.

[140] C. Jarlskog, Z. Phys. C 29, 491 (1985); Phys. Rev. D 35, 1685 (1987).

[141] P. I. Krastev and S. T. Petcov, Phys. Lett. B 205 (1988) 84. 
[142] J. F. Nieves and P. B. Pal, Phys. Rev. D 36, 315 (1987), and Phys. Rev. D 64, 076005 (2001).

[143] G. C. Branco, L. Lavoura and M. N. Rebelo, Phys. Lett. B 180, 264 (1986).

[144] J. A. Aguilar-Saavedra and G. C. Branco, Phys. Rev. D 62, 096009 (2000).

[145] For $\mu \rightarrow e \gamma:$ R. Bolton et al., Phys. Rev. D 38 (1988) 2077; M.L. Brooks et al. [MEGA Collaboration], Phys. Rev. Lett. 83 (1999) 1521, For $\tau \rightarrow \mu \gamma$ : S. Ahmed et al. [CLEO Collaboration], Phys. Rev. D 61 (2000) 071101, hep- For $d_{e}$ : E.D. Commins, S.B. Ross, D. Demille, B.C. Regan, Phys. Rev. A 50 (1994) 2 For $d_{\mu}$ : CERN-Mainz-Daresbury Collaboration, Nucl. Phys. B 150 (1979) 1.

[146] For $\mu \rightarrow e \gamma$ : L.M. Barkov et al., proposal for an experiment at PSI, http://meg.web.psi.ch; For $\tau \rightarrow \mu \gamma$ : D.F. Carvalho, J.R. Ellis, M.E. Gomez, S. Lola and J.C. Romao, hep-ph/0206148; For $d_{e}$ : S.K. Lamoreaux, nucl-ex/0109014, J. Aysto et al., hep-ph/0109217; Y.K. Semertzidis, hep-ex/0401016 For $d_{\mu}$ : R. Carey et al., Letter of Intent to BNL (2000); Y.K. Semertzidis et al., hep-ph/0012087; J. Aysto et al., hep-ph/0109217,

[147] T. P. Cheng and L.-F. Li, Phys. Rev. Lett. 45 (1980) 1908.

[148] T. Appelquist, M. Piai, and R. Shrock, Phys. Lett. B 593, 175 (2004).

[149] T. Appelquist, M. Piai, and R. Shrock, Phys. Lett. B 595, 442 (2004).

[150] F. Gabbiani, E. Gabrielli, A. Masiero, L. Silvestrini, Nucl. Phys. B 477 (1996) 321, hep-ph/9604387. For a recent re-analysis see: I. Masina and C.A. Savoy, Nucl. Phys. B 661 (2003) 365, hep-ph/0211283.

[151] F. Borzumati and A. Masiero, Phys. Rev. Lett. 57 (1986) 961.

[152] R. Barbieri and L.J. Hall, Phys. Lett. B 338 (1994) 212; R. Barbieri, L.J. Hall and A. Strumia, Nucl. Phys. B 445 (1995) 219, hep-ph/9501334.

[153] R. Barbieri, S. Ferrara, C.A. Savoy, Phys. Lett. B 119 (1982) 343; A. Chamsheddine, R. Arnowitt, P. Nath, Phys. Rev. Lett. 49 (1982) 970; L. Hall, J. Lykken, S. Weinberg, Phys. Rev. D 27 (1983) 2359; A. Chamsheddine, R. Arnowitt, P. Nath, N=1 Supergravity, World Scientific, Singapore (1984); N. Ohta, Prog. Theor. Phys. 70 (1983) 542.

[154] J. Hisano, T. Moroi, K. Tobe and M. Yamaguchi, Phys. Rev. D 53 (1996) 2442 [arXiv hep-ph/9510309; S. F. King and M. Oliveira, Phys. Rev. D 60 (1999) 035003 [arXiv:hep-ph/9804283.

[155] S. T. Petcov, S. Profumo, Y. Takanishi and C. E. Yaguna, Nucl. Phys. B 676, 453 (2004) hep-ph/0306195. 
[156] S. Lavignac, I. Masina and C.A. Savoy, Phys. Lett. B 520 (2001) 269, hep-ph/0106245 I. Masina, in Proceedings of SUSY02 Supersymmetry and unification of fundamental interactions, vol.1 331, Hamburg (2002), hep-ph/0210125.

[157] See for instance: J. Sato, K. Tobe and T. Yanagida, Phys. Lett. B 498 (2001) 189, hep-ph/0010348; T. Blazek and S.F. King, Nucl. Phys. B 662 (2003) 359, hep-ph/0211368 M. Ciuchini, A. Masiero, L. Silvestrini, S.K. Vempati, O. Vives, Phys. Rev. Lett. 92 (2004) 071801, hep-ph/0307191; S.M. Barr, Phys. Lett. B 578 (2004) 394, hep-ph/0307372 M.-C. Chen and K. T. Mahanthappa, hep-ph/0409096; hep-ph/0409165.

[158] J.A. Casas and A. Ibarra, Nucl. Phys. B 618 (2001) 171, hep-ph/0103065.

[159] W. Buchmüller, D. Delepine and F. Vissani, Phys. Lett. B 459 (1999) 171, hep-ph/9904219 J. Sato and K. Tobe, Phys. Rev. D 63 (2001) 116010; K.S. Babu, Ts. Enkhbat, I. Gogoladze, Nucl. Phys. B 678 (2004) 233, hep-ph/0308093; I. Masina and C.A. Savoy, hep-ph/0501166.

[160] J. Ellis, M.E. Gomez, G.K. Leontaris, S. Lola and D.V. Nanopoulos, Eur. Phys. J. C14 (2000) 319; J. Hisano and K. Tobe, Phys. Lett. B 510 (2001) 197, hep-ph/0102315.

[161] A. Masiero, S. Profumo, S.K. Vempati, C.E. Yaguna, hep-ph/0401138.

[162] T. Blazek and S. F. King, Phys. Lett. B 518 (2001) 109 [arXiv hep-ph/0105005].

[163] A.Yu. Smirnov, Phys. Rev. D 48 (1993) 3264, hep-ph/9304205; G. Altarelli, F. Feruglio, I. Masina, Phys. Lett. B 472 (2000) 382, hep-ph/9907532.

[164] A. Masiero, S.K. Vempati, O. Vives, Nucl. Phys. B 649 (2003) 189, hep-ph/0209303.

[165] J. R. Ellis, J. Hisano, S. Lola and M. Raidal, Nucl. Phys. B 621, 208 (2002) [arXiv hep-ph/0109125]; J. Ellis, J. Hisano, M. Raidal, Y. Shimizu, Phys. Lett. B 528 (2002) 86, hep-ph/0111324.

[166] I. Masina, Nucl. Phys. B 671 (2003) 432, hep-ph/0304299.

[167] Y. Farzan and M.E. Peskin, hep-ph/0405214.

[168] A. Romanino and A. Strumia, Nucl. Phys. B 622 (2002) 73, hep-ph/0108275.

[169] I. Masina and C.A. Savoy, Phys. Lett. B579 (2004) 99, hep-ph/0309067.

[170] K. S. Babu, B. Dutta and R. N. Mohapatra, Phys. Rev. D 67, 076006 (2003); B. Dutta and R. N. Mohapatra, Phys.Rev. D68, 113008 (2003).

[171] A. D. Sakharov, Pisma Zh. Eksp. Teor. Fiz. 5 (1967) 32 [JETP Lett. 5 (1967 SOPUA,34,392-393.1991 UFNAA,161,61-64.1991) 24].

[172] M. Fukugita and T. Yanagida, Phys. Lett. B174, 45 (1986). 
[173] G. 't Hooft, Phys. Rev. Lett. 37, 8 (1976).

[174] M. Flanz, E. A. Paschos and U. Sarkar, Phys. Lett. B 345 (1995) 248 [Erratum-ibid. B 382 (1996) 447]; L. Covi, E. Roulet and F. Vissani, Phys. Lett. B 384 (1996) 169.

[175] A. Pilaftsis, Phys. Rev. D 56 (1997) 5431; Nucl. Phys. B 504 (1997) 61.

[176] A. Pilaftsis, Int. J. Mod. Phys. A 14, 1811 (1999).

[177] A. Pilaftsis and T. E. J. Underwood, Nucl. Phys. B 692, 303 (2004) [arXiv:hep-ph/0309342].

[178] A. Pilaftsis, Phys. Rev. Lett. 95, 081602 (2005).

[179] A. Pilaftsis and T. E. J. Underwood, arXiv hep-ph/0506107.

[180] E. J. Chun, Phys. Rev. D 69, 117303 (2004); arXiv:hep-ph/0508050

[181] R. Gonzalez Felipe, F. R. Joaquim and B. M. Nobre, Phys. Rev. D 70, 085009 (2004); G. C. Branco, R. Gonzalez Felipe, F. R. Joaquim and B. M. Nobre, arXiv:hep-ph/0507092.

[182] W. Buchmüller and M. Plümacher, Int. J. Mod. Phys. A 15 (2000) 5047

[183] G. F. Giudice et al., Nucl. Phys. B 685, 89 (2004) [arXiv hep-ph/0310123.

[184] V. A. Kuzmin, V. A. Rubakov, and M. E. Shaposhnikov, Phys. Lett. B 155, 36 (1985); J. A. Harvey and M. S. Turner, Phys. Rev. D 42, 3344 (1990).

[185] W. Buchmüller, P. Di Bari and M. Plümacher, hep-ph/0401240.

[186] W. Buchmüller, P. Di Bari and M. Plümacher, Nucl. Phys. B 643 (2002) 367 hep-ph/0205349.

[187] J. R. Ellis, J. Hisano, S. Lola and M. Raidal, Nucl. Phys. B 621, 208 (2002) [arXiv:hep-ph/0109125].

[188] S. Davidson and A. Ibarra, JHEP 0109, 013 (2001) [arXiv:hep-ph/0104076.

[189] M. Fujii, K. Hamaguchi and T. Yanagida, Phys. Rev. D 65, 115012 (2002) [arXiv:hep-ph/0202210].

[190] S. Davidson and A. Ibarra, Phys. Lett. B 535 (2002) 25 hep-ph/0202239.

[191] W. Buchmüller, P. Di Bari and M. Plümacher, Nucl. Phys. B 665 (2003) 445 hep-ph/0302092.

[192] T. Hambye, Y. Lin, A. Notari, M. Papucci and A. Strumia, hep-ph/0312203.

[193] S. Davidson, JHEP 0303 (2003) 037 hep-ph/0302075. 
[194] G. C. Branco, T. Morozumi, B. M. Nobre and M. N. Rebelo, Nucl. Phys. B 617 (2001) 475 hep-ph/0107164; see also M. N. Rebelo, Phys. Rev. D 67, 013008 (2003) hep-ph/0207236.

[195] S. Davidson and R. Kitano, hep-ph/0312007.

[196] P. H. Frampton, S. L. Glashow and T. Yanagida, Phys. Lett. B 548, 119 (2002) hep-ph/0208157; T. Endoh, S. Kaneko, S. K. Kang, T. Morozumi and M. Tanimoto, Phys. Rev. Lett. 89, 231601 (2002) hep-ph/0209020.

[197] R. Kuchimanchi and R. N. Mohapatra, Phys. Rev. D 66, 051301 (2002) hep-ph/0207110; M. Raidal and A. Strumia, Phys. Lett. B 553, 72 (2003) hep-ph/0210021; B. Dutta and R. N. Mohapatra, Phys. Rev. D 68, 056006 (2003) hep-ph/0305059 ; A. Ibarra and G. G. Ross, hep-ph/0312138.

[198] S. F. King, Phys. Rev. D 67, 113010 (2003) hep-ph/0211228.

[199] G. C. Branco, R. Gonzalez Felipe, F. R. Joaquim and M. N. Rebelo, Nucl. Phys. B 640 (2002) 202 hep-ph/0202030; H. B. Nielsen and Y. Takanishi, Nucl. Phys. B 636, 305 (2002) [arXiv:hep-ph/0204027]; M. S. Berger and K. Siyeon, Phys. Rev. D 65 (2002) 053019 hep-ph/0110001; D. Falcone and F. Tramontano, Phys. Rev. D 63 (2001) 073007 hep-ph/0011053.

[200] S. Kaneko and M. Tanimoto, Phys. Lett. B 551 (2003) 127 hep-ph/0210155; S. Kaneko, M. Katsumata and M. Tanimoto, JHEP 0307 (2003) 025 hep-ph/0305014; L. Velasco-Sevilla, JHEP 10 (2003) 035, hep-ph/0307071; V. Barger, D. A. Dicus, H. J. He and T. Li, Phys. Lett. B583 (2004) 173 hep-ph/0310278; W. Rodejohann, Eur. Phys. J. C 32, 235 (2004); B. Dutta and R. N. Mohapatra,hep-ph/0307163; R. N. Mohapatra, S. Nasri and H. B. Yu, Phys. Lett. B 615, 231 (2005) [arXiv:hep-ph/0502026.

[201] S. Antusch and S. F. King, arXiv hep-ph/0508044.

[202] M. Y. Khlopov and A. D. Linde, Phys. Lett. B 138, 265 (1984);

J. R. Ellis, J. E. Kim and D. V. Nanopoulos, Phys. Lett. B 145, 181 (1984);

M. Kawasaki and T. Moroi, Prog. Theor. Phys. 93, 879 (1995) [arXiv:hep-ph/9403364;

M. Kawasaki, K. Kohri and T. Moroi, Phys. Rev. D 63, 103502 (2001) [arXiv:hep-ph/0012279.

[203] T. Moroi, H. Murayama and M. Yamaguchi, Phys. Lett. B 303, 289 (1993);

A. de Gouvêa, T. Moroi and H. Murayama, Phys. Rev. D 56, 1281 (1997) [arXiv:hep-ph/9701244.

[204] R. H. Cyburt, J. R. Ellis, B. D. Fields and K. A. Olive, Phys. Rev. D 67, 103521 (2003) [arXiv astro-ph/0211258;

M. Kawasaki, K. Kohri and T. Moroi, arXiv:astro-ph/0408426.

[205] H. Pagels and J. R. Primack, Phys. Rev. Lett. 48, 223 (1982). 
[206] M. Ibe, R. Kitano, H. Murayama and T. Yanagida, Phys. Rev. D 70, 075012 (2004) [arXiv hep-ph/0403198.

[207] H. P. Nilles, M. Peloso and L. Sorbo, Phys. Rev. Lett. 87, 051302 (2001) [arXiv:hep-ph/0102264;

H. P. Nilles, K. A. Olive and M. Peloso, Phys. Lett. B 522, 304 (2001) [arXiv hep-ph/0107212.

[208] M. Bolz, A. Brandenburg and W. Buchmüller, Nucl. Phys. B 606, 518 (2001) [arXiv hep-ph/0012052.

[209] G. Lazarides and Q. Shafi, Phys. Lett. B 258 (1991) 305;

K. Kumekawa, T. Moroi and T. Yanagida, Prog. Theor. Phys. 92, 437 (1994) [arXiv:hep-ph/9405337;

G. Lazarides, R. K. Schaefer and Q. Shafi, Phys. Rev. D 56, 1324 (1997) [arXiv,hep-ph/9608256;

G. Lazarides, Springer Tracts Mod. Phys. 163, 227 (2000) [arXiv/hep-ph/9904428];

G. F. Giudice, M. Peloso, A. Riotto and I. Tkachev, JHEP 9908, 014 (1999) [arXiv hep-ph/9905242;

T. Asaka, K. Hamaguchi, M. Kawasaki and T. Yanagida, Phys. Lett. B 464, 12 (1999) [arXiv:hep-ph/9906366;

T. Asaka, K. Hamaguchi, M. Kawasaki and T. Yanagida, Phys. Rev. D 61, 083512 (2000) [arXiv:hep-ph/9907559];

M. Kawasaki, M. Yamaguchi and T. Yanagida, Phys. Rev. D 63, 103514 (2001) [arXiv:hep-ph/0011104].

[210] H. Murayama, H. Suzuki, T. Yanagida and J. Yokoyama, Phys. Rev. Lett. 70, 1912 (1993);

H. Murayama and T. Yanagida, Phys. Lett. B 322, 349 (1994) [arXiv hep-ph/9310297;

K. Hamaguchi, H. Murayama and T. Yanagida, Phys. Rev. D 65 (2002) 043512 hep-ph/0109030;

for a review see, e.g., K. Hamaguchi, arXiv hep-ph/0212305

[211] M. Fujii, M. Ibe and T. Yanagida, Phys. Lett. B 579, 6 (2004) [arXiv:hep-ph/0310142;

J. L. Feng, S. Su and F. Takayama, Phys. Rev. D 70, 075019 (2004);

L. Roszkowski and R. Ruiz de Austri, arXiv:hep-ph/0408227.

[212] W. Buchmüller, K. Hamaguchi and M. Ratz, Phys. Lett. B 574, 156 (2003) [arXiv:hep-ph/0307181;

W. Buchmüller, K. Hamaguchi, O. Lebedev and M. Ratz, Nucl. Phys. B 699, 292 (2004) [arXiv:hep-th/0404168];

R. Kallosh and A. Linde, JHEP 0412, 004 (2004) [arXiv/hep-th/0411011;

W. Buchmüller, K. Hamaguchi, O. Lebedev and M. Ratz, JCAP 0501, 004 (2005) [arXiv:hep-th/0411109]. 
[213] M. Bolz, W. Buchmüller and M. Plümacher, Phys. Lett. B 443, 209 (1998) [arXiv:hep-ph/9809381;

M. Fujii and T. Yanagida, Phys. Lett. B 549, 273 (2002) [arXiv/hep-ph/0208191;

M. Fujii, M. Ibe and T. Yanagida, Phys. Rev. D 69, 015006 (2004) [arXiv:hep-ph/0309064;

J. R. Ellis, D. V. Nanopoulos and S. Sarkar, Nucl. Phys. B 259, 175 (1985).

[214] W. Buchmüller, K. Hamaguchi, M. Ratz and T. Yanagida, Phys. Lett. B 588, 90 (2004) [arXiv:hep-ph/0402179];

G. Weiglein et al., arXiv/hep-ph/0410364.

[215] P. J. O'Donnell and U. Sarkar, Phys. Rev. D 49, 2118 (1994) hep-ph/9307279].

[216] G. Lazarides and Q. Shafi, Phys. Rev. D 58, 071702 (1998).

[217] T. Hambye and G. Senjanovic, Phys. Lett. B 582, 73 (2004).

[218] S. Antusch and S. F. King, Phys. Lett. B 597, (2004) 199, hep-ph/0405093.

[219] A. S. Joshipura and E. A. Paschos, hep-ph/9906498 A. S. Joshipura, E. A. Paschos and W. Rodejohann, Nucl. Phys. B 611, 227 (2001).

[220] A. S. Joshipura, E. A. Paschos and W. Rodejohann, JHEP 0108, 029 (2001); W. Rodejohann, Phys. Lett. B 542, 100 (2002).

[221] S. Nasri, J. Schechter and S. Moussa, hep-ph/0402176.

[222] W. Rodejohann, Phys. Rev. D 70, 073010 (2004) hep-ph/0403236; P. h. Gu and X. j. Bi, hep-ph/0405092; N. Sahu and S. Uma Sankar, arXiv/hep-ph/0406065. W. l. Guo, hep-ph/0406268.

[223] E. J. Chun and S. K. Kang, Phys. Rev. D 63, 097902 (2001).

[224] T. Dent, G. Lazarides and R. Ruiz de Austri, hep-ph/0312033.

[225] E. Ma and U. Sarkar, Phys. Rev. Lett. 80, 5716 (1998).

[226] T. Hambye, E. Ma and U. Sarkar, Nucl. Phys. B 602, 23 (2001).

[227] G. Lazarides, Phys. Lett. B 452, 227 (1999).

[228] R. N. Mohapatra, A. Perez-Lorenzana and C. A. de Sousa Pires, Phys. Lett. B 474, $355(2000)$.

[229] K. Dick, M. Lindner, M. Ratz and D. Wright, Phys. Rev. Lett. 84, 4039 (2000) hep-ph/9907562.

[230] B. A. Campbell, S. Davidson, J. R. Ellis and K. A. Olive, Phys. Lett. B 297, 118 (1992) [arXiv hep-ph/9302221]. 
[231] N. Arkani-Hamed, L. J. Hall, H. Murayama, D. R. Smith and N. Weiner, Phys. Rev. D 64, 115011 (2001) hep-ph/0006312.

[232] F. Borzumati and Y. Nomura, Phys. Rev. D 64, 053005 (2001) hep-ph/0007018.

[233] H. Murayama and A. Pierce, Phys. Rev. Lett. 89, 271601 (2002) hep-ph/0206177.

[234] M. Boz and N. K. Pak, Eur. Phys. J. C 37, 507 (2004).

[235] W. Buchmüller, P. Di Bari and M. Plümacher, Phys. Lett. B 547 (2002) 128.

[236] C. H. Albright and S. M. Barr, hep-ph/0312224.

[237] G. D'Ambrosio, G. F. Giudice and M. Raidal, Phys. Lett. B 575 (2003) 75; Y. Grossman, T. Kashti, Y. Nir and E. Roulet, Phys. Rev. Lett. 91 (2003) 251801.

[238] T. Hambye, J. March-Russell and S. M. West, hep-ph/0403183.

[239] R. N. Mohapatra and X. M. Zhang, Phys. Rev. D 46, 5331 (1992); L. Boubekeur, T. Hambye and G. Senjanović, Phys. Rev. Lett. 93 (2004) 111601 [arXiv:hep-ph/0404038; N. Sahu and U. Yajnik, hep-ph/0410075; S. F. King and T. Yanagida, hep-ph/0411030.

[240] K. S. Babu and R. N. Mohapatra, Phys. Lett. B 267, 400 (1991).

[241] S. F. King, Phys. Lett. B 439, 350 (1998) hep-ph/9806440; S. F. King, Nucl. Phys. B 562, 57 (1999) hep-ph/9904210|; S. F. King, Nucl. Phys. B 576 (2000) 85 hep-ph/9912492; S. F. King, JHEP 0209, 011 (2002) hep-ph/0204360; for a review see S. Antusch and S. F. King, New J. Phys. 6 (2004) 110 hep-ph/0405272.

[242] E. K. Akhmedov, M. Frigerio and A. Y. Smirnov, JHEP 0309, 021 (2003) [arXiv:hep-ph/0305322].

[243] S. F. King and G. G. Ross, Phys. Lett. B 520 (2001) 243 [arXiv hep-ph/0108112]; S. F. King and G. G. Ross, Phys. Lett. B 574 (2003) 239 [arXiv hep-ph/0307190].

[244] S. Antusch and S. F. King, arXiv:hep-ph/0507333.

[245] M. Hirsch and S. F. King, Phys. Rev. D 64 (2001) 113005 [arXiv hep-ph/0107014].

[246] H. Murayama, H. Suzuki, T. Yanagida and J. Yokoyama, Phys. Rev. Lett. 70 (1993) 1912; J. R. Ellis, M. Raidal and T. Yanagida, Phys. Lett. B 581, 9 (2004) hep-ph/0303242.

[247] S. Antusch, M. Bastero-Gil, S. F. King and Q. Shafi, hep-ph/0411298.

[248] S. Antusch and S. F. King, Nucl. Phys. B 705 (2005) 239, hep-ph/0402121.

[249] For a review, see S. Raby, Particle Data group book: Phys. Rev. D 66, 010001 (2002). 
[250] H. Murayama and A. Pierce, Phys. Rev. D65, 055009 (2002).

[251] K. S. Babu and R. N. Mohapatra, Phys. Rev. Lett. 70, 2845 (1993).

[252] D. G. Lee and R. N. Mohapatra, Phys. Rev. D 51, 1353 (1995).

[253] K. Matsuda, Y. Koide and T. Fukuyama, Phys. Rev. D 64, 053015 (2001) hep-ph/0010026; K. Matsuda, hep-ph/0401154 K. Matsuda, Y. Koide, T. Fukuyama and H. Nishiura, Phys. Rev. D 65, 033008 (2002); T. Fukuyama and N. Okada, JHEP 0211, 011 (2002); B. Bajc, G. Senjanovic and F. Vissani, hep-ph/0402140; B. Dutta, Y. Mimura and R. N. Mohapatra, Phys.Rev. D69, 115014 (2004) ; Phys.Lett. B603, 35 (2004); Phys.Rev.Lett.94, 091804 (2005); hep-ph/0507319; T. Fukuyama, A. Ilakovac, T. Kikuchi, S. Meljanac and N. Okada, hep-ph/0401213, hep-ph/0405300, B. Bajc, A. Melfo, G. Senjanovic and F. Vissani, Phys. Rev. D 70, 035007 (2004); C. S. Aulakh and A. Girdhar, hep-ph/0204097; S. Bertolini and M. Malinsky, Phys. Rev. D 72, 055021 (2005) [arXiv/hep-ph/0504241];

[254] B. Bajc, G. Senjanovic and F. Vissani, Phys. Rev. Lett. 90, 051802 (2003); H. S. Goh, R. N. Mohapatra and S. P. Ng, Phys. Lett. B 570, 215 (2003) hep-ph/0303055; H. S. Goh, R. N. Mohapatra and S. P. Ng, Phys. Rev. D68, 115008 (2003) [hep-ph/0308197].

[255] K. S. Babu, J. C. Pati and F. Wilczek, hep-ph/9812538, Nucl. Phys. B566, 33 (2000); C. Albright and S. M. Barr, Phys. Rev. Lett. 85, 244 (2001); T. Blazek, S. Raby and K. Tobe, Phys. Rev. D62, 055001 (2000); Z. Berezhiani and A. Rossi, Nucl. Phys. B594, 113 (2001); R. Kitano and Y. Mimura, Phys. Rev. D63, 016008 (2001); for a recent review, see C. Albright, hep-ph/0212090.

[256] M.-C. Chen and K. T. Mahanthappa, Int. J. Mod. Phys. A 18, 5819 (2003); AIP Conf. Proc. 721, 269 (2004).

[257] M.-C. Chen and K. T. Mahanthappa, hep-ph/0411158

[258] G. C. Branco, P. A. Parada, M. N. Rebelo, hep-ph/0307119.

[259] Y. Achiman, Phys. Lett. B599, 75 (2004).

[260] P. H. Chankowski and Z. Pluciennik, Phys. Lett. B 316, 312 (1993) [arXiv,hep-ph/9306333.

[261] S. Antusch, M. Drees, J. Kersten, M. Lindner and M. Ratz, Phys. Lett. B 519, 238 (2001) [arXiv:hep-ph/0108005].

[262] S. Antusch, M. Drees, J. Kersten, M. Lindner and M. Ratz, Phys. Lett. B 525, 130 (2002) [arXiv hep-ph/0110366].

[263] S. Antusch and M. Ratz, JHEP 07 (2002), 059 [arXiv hep-ph/0203027]. 
[264] P. H. Chankowski, W. Krolikowski, and S. Pokorski, Phys. Lett. B473 (2000), 109 [arXiv:hep-ph/9910231.

[265] J. A. Casas, J. R. Espinosa, A. Ibarra, and I. Navarro, Nucl. Phys. B573 (2000), 652 [arXiv hep-ph/9910420].

[266] S. Antusch, J. Kersten, M. Lindner, and M. Ratz, Nucl. Phys. B674 (2003), 401 [arXiv:hep-ph/0305273.

[267] J. w. Mei and Z. z. Xing, Phys. Rev. D 69, 073003 (2004) [arXiv hep-ph/0312167].

[268] S. Luo, J. w. Mei and Z. z. Xing, Phys. Rev. D 72, 053014 (2005) [arXiv:hep-ph/0507065].

[269] J. R. Ellis and S. Lola, Phys. Lett. B 458, 310 (1999) [arXiv hep-ph/9904279].

[270] J. A. Casas, J. R. Espinosa, A. Ibarra and I. Navarro, Nucl. Phys. B 556, 3 (1999) [arXiv:hep-ph/9904395.

[271] J. A. Casas, J. R. Espinosa, A. Ibarra and I. Navarro, Nucl. Phys. B 569, 82 (2000) [arXiv:hep-ph/9905381.

[272] R. Adhikari, E. Ma and G. Rajasekaran, Phys. Lett. B 486, 134 (2000) [arXiv:hep-ph/0004197.

[273] A. S. Joshipura and S. D. Rindani, Phys. Lett. B 494, 114 (2000) [arXiv:hep-ph/0007334.

[274] E. J. Chun, Phys. Lett. B 505, 155 (2001) [arXiv:hep-ph/0101170.

[275] A. S. Joshipura, S. D. Rindani and N. N. Singh, Nucl. Phys. B 660, 362 (2003) [arXiv:hep-ph/0211378.

[276] A. S. Joshipura and S. D. Rindani, Phys. Rev. D 67, 073009 (2003) [arXiv:hep-ph/0211404.

[277] N. N. Singh and M. K. Das, (2004), hep-ph/0407206.

[278] N. Haba and N. Okamura, Eur. Phys. J. C 14, 347 (2000) [arXiv hep-ph/9906481].

[279] T. Miura, E. Takasugi and M. Yoshimura, Prog. Theor. Phys. 104, 1173 (2000) [arXiv:hep-ph/0007066].

[280] N. Haba, Y. Matsui, and N. Okamura, Eur. Phys. J. C17, 513 (2000) [arXiv hep-ph/0005075]; N. Haba, Y. Matsui, N. Okamura, and M. Sugiura, Prog. Theor. Phys. 103, 145 (2000) [arXiv hep-ph/9908429.

[281] P. H. Chankowski and S. Pokorski, Int. J. Mod. Phys. A 17, 575 (2002) [arXiv:hep-ph/0110249. 
[282] T. Miura, T. Shindou, and E. Takasugi, Phys. Rev. D66 (2002), 093002 [arXiv:hep-ph/0206207.

[283] C. W. Chiang, Phys. Rev. D 63, 076009 (2001) [arXiv:hep-ph/0011195].

[284] M. Lindner, M. Ratz and M. A. Schmidt, arXiv:hep-ph/0506280.

[285] S. F. King and N. N. Singh, Nucl. Phys. B591 (2000), 3-25 [arXiv hep-ph/0006229].

[286] S. Antusch, J. Kersten, M. Lindner, and M. Ratz, Phys. Lett. B538 (2002), 87 [arXiv:hep-ph/0203233.

[287] S. Antusch, J. Kersten, M. Lindner, and M. Ratz, Phys. Lett. B544 (2002), 1 [arXiv:hep-ph/0206078.

[288] J.-w. Mei and Z.-z. Xing, Phys. Rev. D70 (2004), 053002 [arXiv hep-ph/0404081.

[289] J. Ellis, A. Hektor, M. Kadastik, K. Kannike and M. Raidal, arXiv:hep-ph/0506122.

[290] S. Antusch, J. Kersten, M. Lindner, M. Ratz and M. A. Schmidt, JHEP 0503, 024 (2005) [arXiv:hep-ph/0501272.

[291] J. w. Mei, Phys. Rev. D 71, 073012 (2005) [arXiv hep-ph/0502015.

[292] G. Dutta, arXiv:hep-ph/0203222.

[293] G. Bhattacharyya, A. Raychaudhuri and A. Sil, Phys. Rev. D 67 (2003), 073004 [arXiv:hep-ph/0211074.

[294] T. Miura, T. Shindou and E. Takasugi, Phys. Rev. D 68 (2003), 093009 [arXiv:hep-ph/0308109.

[295] T. Shindou and E. Takasugi, Phys. Rev. D 70 (2004), 013005 [arXiv:hep-ph/0402106].

[296] J. A. Casas, J. R. Espinosa and I. Navarro, Phys. Rev. Lett. 89 (2002), 161801 [arXiv:hep-ph/0206276].

[297] J. A. Casas, J. R. Espinosa, and I. Navarro, JHEP 09 (2003), 048 [arXiv:hep-ph/0306243.

[298] P. H. Chankowski, A. Ioannisian, S. Pokorski, and J. W. F. Valle, Phys. Rev. Lett. 86 (2001), 3488 [arXiv:hep-ph/0011150].

[299] M.-C. Chen and K. T. Mahanthappa, Int. J. Mod. Phys. A 16, 3923 (2001) [arXiv:hep-ph/0102215].

[300] K. S. Babu, E. Ma and J. W. F. Valle, Phys. Lett. B 552 (2003), 207 [arXiv:hep-ph/0206292].

[301] N. Haba, Y. Matsui, N. Okamura and T. Suzuki, Phys. Lett. B 489 (2000), 184 [arXiv:hep-ph/0005064. 
[302] T. K. Kuo, S. H. Chiu and G. H. Wu, Eur. Phys. J. C 21 (2001), 281 [arXiv:hep-ph/0011058.

[303] R. Gonzalez Felipe and F. R. Joaquim, JHEP 0109 (2001), 015 [arXiv hep-ph/0106226].

[304] M. K. Parida, C. R. Das and G. Rajasekaran, Pramana 62, 647 (2004) [arXivihep-ph/0203097.

[305] K. R. S. Balaji, A. S. Dighe, R. N. Mohapatra, and M. K. Parida, Phys. Lett. B481 (2000), 33 [arXiv:hep-ph/0002177].

[306] K. R. S. Balaji, R. N. Mohapatra, M. K. Parida and E. A. Paschos, Phys. Rev. D 63, 113002 (2001) [arXiv:hep-ph/0011263].

[307] K. S. Babu and R. N. Mohapatra, Phys. Lett. B532 (2002), 77 [arXiv hep-ph/0201176].

[308] S. Antusch, P. Huber, J. Kersten, T. Schwetz and W. Winter, Phys. Rev. D 70 (2004) 097302, hep-ph/0404268.

[309] W. Grimus and L. Lavoura, arXiv/hep-ph/0410279.

[310] R. Barbieri, P. Creminelli, A. Strumia and N. Tetradis, Nucl. Phys. B 575, 61 (2000) hep-ph/9911315v3].

[311] W. Buchmüller and M. Plümacher, Phys. Lett. B 511, 74 (2001) hep-ph/0104189.

[312] D.W.Liu et al [Super-Kamiokande collaboration], hep-ex/0402015

[313] Z. Paraktchieva et al [MUNU collaboration], Phys Lett B 564, 190 (2003); Phys.Lett.B615:153-159,2005.

[314] H.B.Li et al [Texono collaboration], Phys Rev Lett ,90 , (2003).

[315] H.O. Back et al, Phys. Lett. B 563, 35 (2003).

[316] J.F. Beacom and P.Vogel, Phys Rev Lett, 83,5222 (1999).

[317] A.S. Joshipura and S. Mohanty, Phys. Rev. D 66, 012003 (2002).

[318] W. Grimus et al, Nucl Phys B 648, 376 (2003).

[319] A.J. Grifols, E. Masso and S. Mohanty, hep-ph/0401144

[320] J.N. Bahcall and M.H. Pinsonneault, astro-ph/0402114.

[321] L. Wolfenstein, Phys. Rev. D 17 (1978) 2369.

[322] M. Guzzo, A. Masiero and S. T. Petcov, Phys. Lett. B260 (1991) 154. 
[323] N. Fornengo, M. Maltoni, R. Tomas Bayo, J. W. F. Valle, Phys. Rev. D 65013010 (2002).

[324] A. Friedland, C. Lunardini and M. Maltoni, Phys. Rev. D 70, 111301 (2004); Phys.Rev. D 72, 053009 (2005); hep-ph/0506143.

[325] M. M. Guzzo, P. C. de Holanda, O. L. G. Peres, hep-ph/0403134.

[326] Alexander Friedland, Cecilia Lunardini, Carlos Peña-Garay, Phys. Lett. B 594, 347 (2004) [arXiv:hep-ph/0402266.

[327] S. Bergmann, Y. Grossman, D. M. Pierce Phys. Rev. D61 053005 (2000).

[328] S. Bergmann, M. M. Guzzo, P. C. de Holanda, P. I. Krastev, H. Nunokawa Phys. Rev. D 62, 073001 (2000).

[329] Zurab Berezhiani, Anna Rossi, Phys. Lett. B 535, 207 (2002).

[330] S. Davidson, C. Peña-Garay, N. Rius, A. Santamaria JHEP 0303 (2003) 011

[331] M. Cirelli, G. Marandella, A. Strumia and F. Vissani, hep-ph/0403158.

[332] T.D. Lee and C.N. Yang, Phys. Rev. 104, 254 (1956), A. Salam, Nuovo Cim. 5, 299 (1957), V. Kobzarev, L. Okun, and I. Pomeranchuk, Sov.J.Nucl.Phys. 3, 837 (1966).

[333] L.B. Okun, Sov.Phys. JETP 52, 351 (1980), S.I. Blinnikov and M. Yu. Khlopov, Sov. Astron. Jour. 60, 632 (1983), B. Holdom, Phys. Lett. B166, 196 (1985), S.L. Glashow, Phys. Lett. B167, 35 (1986), E.D. Carlson and S.L. Glashow, Phys. Lett. B193, 168 (1987), M.Yu. Khlopov et al., Sov. Astron. Jour. 68, 42 (1991), E. Kolb, D. Seckel and M. Turner, Nature, 514, 415 (1985), Z.K. Silagadze, Mod. Phys. Lett. A 14, 2321 (1999) and Acta Phys. Polon. B 32, 99 (2001).

[334] R. Foot, H. Lew and R.R. Volkas, Phys. Lett. B271, 67 (1991) and Mod. Phys. Lett. A7, 2567 (1992), R. Foot, Mod. Phys. Lett. A9, 169 (1994), R. Foot and R.R. Volkas, Phys. Rev. D 52, 6595 (1995).

[335] Z. G. Berezhiani and R. N. Mohapatra, Phys. Rev. D 52, 6607 (1995), Z.G. Berezhiani, A.D. Dolgov and R.N. Mohapatra, Phys. Lett. B375, 26 (1996), Z.G. Berezhiani, Acta Phys. Polonica, B27, 1503 (1996), R.N. Mohapatra and V.L. Teplitz, Astrophys. J. 478, 29 (1997), Z. Berezhiani, D. Comelli and F.L. Villante, Phys. Lett. B503, 362 (2001).

[336] K. Benakli and A. Y. Smirnov, Phys. Rev. Lett. 79, 4314 (1997)

[337] Z. Chacko and R. N. Mohapatra, Phys. Rev. D 61, 053002 (2000) [arXiv:hep-ph/9905388; M. Frank, M. Sher and I. Turan, arXiv hep-ph/0412090; hep-ph/0503084.

[338] R. N. Mohapatra and A. Perez-Lorenzana, Nucl. Phys. B 576, 466 (2000) 
[339] V. Berezinsky, M. Narayan and F. Vissani, hep-ph/0401029.

[340] G. Cacciapaglia, M. Cirelli and A. Romanino, Phys. Rev. D 68, 033013 (2003); For a review, see R. Volkas, Prog. in Part. and Nucl. Phys. 48, 161 (2002).

[341] S. M. Bilenky, C. Giunti and W. Grimus, Eur. Phys. J. C 1, 247 (1998)

[342] A. Strumia, Phys. Lett. B 539, 91 (2002)

[343] S. M. Bilenky, S. Pascoli and S. T. Petcov, Phys. Rev. D 64, 113003 (2001) [arXiv:hep-ph/0104218; R. N. Mohapatra, S. Nasri and H. B. Yu, Phys. Rev. D 72, 033007 (2005).

[344] S.W. Allen, R.W. Schmidt and S.L. Bridle, Mon. Not. Roy. Astron. Soc. 346, 593 (2003)

[345] R. N. Mohapatra and S. Nasri, hep-ph/0407194.

[346] V. Barger, J. P. Kneller, P. Langacker, D. Marfatia and G. Steigman, Phys. Lett. B 569, 123 (2003) [arXiv hep-ph/0306061].

[347] G. Gelmini, S. Palomares-Ruiz and S. Pascoli, Phys. Rev. Lett. 93, 081302 (2004) [arXiv astro-ph/0403323.

[348] V. S. Berezinsky and A. Vilenkin, Phys. Rev. D 62, 083512 (2000)

[349] M. Maltoni, T. Schwetz, M. A. Tortola and J. W. F. Valle, hep-ph/0305312, hep-ph/0405172.

[350] M.C. Gonzalez-Garcia, M. Maltoni, C. Pena-Garay, Phys. Rev. D64, 093001 (2001); M. Maltoni, T. Schwetz, M. Tórtola and J. W. F. Valle, Nucl. Phys. B643, 321 (2002); H. Päs, L. Song, T. Weiler, Phys. Rev. D67, 073019 (2003).

[351] A. Donini, M. Lusignoli, D. Meloni, Nucl. Phys. B624, 405 (2002).

[352] S. Pakvasa, P. Roy, Phys. Lett. B535, 181 (2002).

[353] R.E. Shrock, Phys. Lett. B96, 159 (1980); Phys. Rev. D24, 1232 (1981).

[354] R. E. Shrock, Phys. Rev. D24, 1275 (1981); Phys. Lett. B112, 382 (1982).

[355] R. Abela et al., Phys. Lett. B105, 263 (1981); F. Calaprice et al., Phys. Lett. B106, 175 (1981); R. Minehart et al., Phys. Rev. Lett. 52, 804 (1984); M. Daum et al., Phys. Rev. D36, 2624 (1987).

[356] Y. Asano et al., Phys. Lett. B104, 84 (1981); R. Hayano et al., Phys. Rev. Lett. 49, 1305 (1982).

[357] D. Bryman et al., Phys. Rev. Lett. 50, 1546 (1983); G. Azuelos et al., Phys. Rev. Lett. 56, 2241 (1986); N. De Leener-Rosier et al., Phys. Rev. D43, 3611 (1991); D. Britton et al., Phys. Rev. D46, R885 (1992). 
[358] T. Yamazaki, in the Neutrino-84 Conference.

[359] B. Armbruster et al., Phys. Lett. B348, 19 (1995).

[360] D. Bryman and T. Numao, Phys. Rev. D53, 558 (1996); J. Formaggio et al., Phys. Rev. Lett. 84, 443 (2000); M. Daum et al., Phys. Rev. Lett. 85, 1515 (2000) P. Astier, Phys. Lett. B527, 23 (2002).

[361] D. Britton et al., Phys. Rev. Lett. 68, 3000 (1992).

[362] M. Nakagawa, H. Okonagi, S. Sakata, and A. Toyoda, Prog. Theor. Phys. 30, 727 (1963).

[363] B. McKellar, Phys. Lett. B97, 93 (1980).

[364] I. Kobzarev, B. Martemyanov, L. Okun, and M. Schepkin, Yad. Fiz. 32, 1590 (1980) [Sov. J. Nucl. Phys. 32, 823 (1980)].

[365] A. De Rujula, Nucl. Phys. B188, 414 (1981).

[366] J. Deutsch, M. Lebrun, and R. Prieels, Phys. Rev. D27, 1644 (1983).

[367] J. H. Missimer, R. N. Mohapatra, and N. C. Mukhopahyay, Phys. Rev. D 50, 2067 (1994).

[368] L. Littenberg and R. Shrock, Phys. Rev. Lett. 68443 (1992).

[369] R. Appel et al., Phys. Rev. Lett. 85, 2877 (2000).

[370] L. Littenberg and R. Shrock, Phys. Lett. B491, 285-290 (2000).

[371] C. Dib, V. Gribanov, S. Kovalenko and M. Schmidt, Phys. Lett. B 493, 82 (2000).

[372] A. Belyaev et al., hep-ph/0107046.

[373] Two recent reviews on rare $K$ decays include L. Littenberg, hep-ex/0201026 and J. Rosner and B. Winstein, eds., Kaon Physics (University of Chicago Press, Chicago, 2001);

[374] A. Weir et al., Phys. Rev. D 41, 1384 (1990).

[375] P. Avery et al., Phys. Lett. B 223, 470 (1989).

[376] L. Littenberg and R. Shrock, Phys. Rev. D46, R892 (1992).

[377] D. Rajaram et al., Phys. Rev. Lett. 94, 181801 (2005).

[378] R. E. Shrock, Phys. Lett. B 96, 159 (1980); Phys. Rev. D24, 1232, 1275 (1981); Phys. Lett. B 112, 382 (1982).

[379] M. Gronau, Phys. Rev. D28, 2762 (1983). 
[380] M. Gronau, C. N. Leung, and J. L. Rosner, Phys. Rev. D 29, 2539 (1984).

[381] Some early accelerator experiments include F. Bergsma et al., Phys. Lett. B128, 361 (1983); A. Cooper-Sarkar et al., B160, 267 (1985); J. Dorenbos et al., Phys. Lett. B166, 473 (1986); G. Bernardi et al., Phys. Lett. B166, 479 (1986); L. Oberauer et al., Phys. Lett. B198, 113 (1987); G. Bernardi et al., Phys. Lett. B203, 332 (1988). A more recent search is A. Vaitaitis et al., Phys. Rev. Lett. 83, 4943 (1999). See [3] for further references.

[382] A. Kusenko, S. Pascoli and D. Semikoz, arXiv hep-ph/0405198.

[383] S. Dodelson, L. M. Widrow, Phys. Rev. Lett. 72, 17 (1994).

[384] X. Shi, G. Fuller, Phys. Rev. Lett. 82, 2832 (1999)

[385] K. Abazajian, G. Fuller, M. Patel, Phys. Rev. D64, 023501 (2001).

[386] A.D. Dolgov, S.H. Hansen, G. Raffelt, D.V. Semikoz, Nucl. Phys. B590, 562 (2000).

[387] A. Kusenko, G. Segre, Phys. Lett. B396, 197 (1997); G. Fuller, A. Kusenko, I. Mocioiu, S. Pascoli, Phys. Rev. D68, 103002 (2003).

[388] Y. Grossman and S. Rakshit, Phys. Rev. D 69, 093002 (2004), hep-ph/0311310.

[389] Y. Grossman and H. E. Haber, Phys. Rev. D 59, 093008 (1999) hep-ph/9810536.

[390] A. S. Joshipura and M. Nowakowski, Phys. Rev. D 51, 2421 (1995) hep-ph/9408224

[391] S. Davidson and M. Losada, JHEP 0005, 021 (2000) hep-ph/0005080.

[392] S. Davidson and M. Losada, Phys. Rev. D 65, 075025 (2002) hep-ph/0010325.

[393] R. N. Mohapatra, Prog. in Part. and Nucl. Phys. 31, 39 (1993).

[394] T. Banks, Y. Grossman, E. Nardi and Y. Nir, Phys. Rev. D 52, 5319 (1995) hep-ph/9505248 ; M. Nowakowski and A. Pilaftsis, Nucl. Phys. B 461, 19 (1996) hep-ph/9508271.

[395] N. Arkani-Hamed, L. J. Hall, H. Murayama, D. R. Smith and N. Weiner, hep-ph/0007001.

[396] F. Borzumati, K. Hamaguchi, Y. Nomura and T. Yanagida, hep-ph/0012118

[397] S. Abel, A. Dedes and K. Tamvakis, hep-ph/0402287.

[398] J. March-Russell and S. West, hep-ph/0403067.

[399] Y. Grossman and H. E. Haber, Phys. Rev. Lett. 78, 3438 (1997) hep-ph/9702421.

[400] N. V. Krasnikov, Phys. Lett. B 388, 783 (1996) hep-ph/9511464. 
[401] N. Arkani-Hamed, H. C. Cheng, J. L. Feng and L. J. Hall, Phys. Rev. Lett. 77, 1937 (1996) hep-ph/9603431.

[402] N. Arkani-Hamed, J. L. Feng, L. J. Hall and H. C. Cheng, Nucl. Phys. B 505, 3 (1997) hep-ph/9704205.

[403] M. Dine, Y. Grossman and S. Thomas, eConf C010630, P332 (2001) [Int. J. Mod. Phys. A 18, 2757 (2003)] hep-ph/0111154.

[404] For a recent discussion, see P. Langacker, hep-ph/0308033.

[405] M. Dine, V. Kaplunovsky, M. L. Mangano, C. Nappi and N. Seiberg, Nucl. Phys. B 259, 549 (1985).

[406] J. D. Breit, B. A. Ovrut and G. C. Segre, Phys. Lett. B 158, 33 (1985).

[407] E. Witten, Nucl. Phys. B 268, 79 (1986).

[408] R. N. Mohapatra, see Ref. [90].

[409] R. N. Mohapatra and J. W. F. Valle, see vRef. [90].

[410] S. Nandi and U. Sarkar, Phys. Rev. Lett. 56, 564 (1986).

[411] G. Cleaver, M. Cvetic, J. R. Espinosa, L. L. Everett and P. Langacker, Phys. Rev. D 57, 2701 (1998) hep-ph/9705391.

[412] M. Cvetic and P. Langacker, Phys. Rev. D 46, 2759 (1992) hep-th/9205029.

[413] D. Mochinaga, Phys. Lett. B 312, 405 (1993).

[414] A. E. Faraggi and E. Halyo, Phys. Lett. B 307, 311 (1993) hep-th/9303060|.

[415] R. Blumenhagen, M. Cvetic, P. Langacker and G. Shiu, arXiv:hep-th/0502005.

[416] L. E. Ibanez, F. Marchesano and R. Rabadan, JHEP 0111, 002 (2001).

[417] I. Antoniadis, E. Kiritsis, J. Rizos and T. N. Tomaras, Nucl. Phys. B 660, 81 (2003).

[418] A. Font, L. E. Ibanez, F. Quevedo and A. Sierra, Nucl. Phys. B 331, 421 (1990).

[419] C. Coriano and A. E. Faraggi, Phys. Lett. B 581, 99 (2004).

[420] J. R. Ellis, G. K. Leontaris, S. Lola and D. V. Nanopoulos, Phys. Lett. B 425, 86 (1998).

[421] J. R. Ellis, G. K. Leontaris, S. Lola and D. V. Nanopoulos, Eur. Phys. J. C 9, 389 (1999).

[422] J. E. Kim, Phys. Lett. B 591, 119 (2004) [arXiv hep-ph/0403196.

[423] T. Kobayashi, S. Raby and R. J. Zhang, Nucl. Phys. B 704, 3 (2005) [arXiv hep-ph/0409098. 
[424] J. Giedt, G. L. Kane, P. Langacker and B. D. Nelson, Phys. Rev. D 71, 115013 (2005) [arXiv:hep-th/0502032.

[425] S. Antusch, O. J. Eyton-Williams and S. F. King, JHEP 0508 (2005) 103 [arXiv:hep-ph/0505140.

[426] P. Langacker and B. D. Nelson, Phys. Rev. D 72, 053013 (2005) [arXiv:hep-ph/0507063.

[427] M. Cvetic and P. Langacker, Phys. Rev. D 54, 3570 (1996) hep-ph/9511378.

[428] C. T. Hill and E. H. Simmons, Phys. Rept. 381, 235 (2003) [Erratum-ibid. 390, 553 (2004)] hep-ph/0203079.

[429] M. Cvetic and P. Langacker, in Perspectives in Supersymmetry (World Scientific, Singapore, 1998), ed. G. L. Kane, p 312, hep-ph/9707451.

[430] J. Erler, P. Langacker and T. j. Li, Phys. Rev. D 66, 015002 (2002) hep-ph/0205001.

[431] For recent reviews, see P. Langacker, Int. J. Mod. Phys. A 18, 4015 (2003) hep-ph/0304053, and hep-ph/0402203.

[432] J. Kang, P. Langacker, and T. Li, Phys. Rev. D 71, 015012 (2005) [arXiv:hep-ph/0411404.

[433] K. A. Olive, D. N. Schramm and G. Steigman, Nucl. Phys. B 180, 497 (1981).

[434] V. Barger, P. Langacker and H. S. Lee, Phys. Rev. D 67, 075009 (2003) hep-ph/0302066.

[435] S. Weinberg, Phys. Rev. D 19, 1277 (1979); L. Susskind, ibid. D 20, 2619 (1979).

[436] Earlier work on dynamical symmetry breaking of gauge symmetries includes R. Jackiw and K. Johnson, Phys. Rev. D 8, 2386 (1973); J. Cornwall and R. Norton, ibid. D 8, 3338 (1973); M. Weinstein, Phys. Rev. D 7, 1854 (1973); S. Weinberg, ibid. D 13, 974 (1976).

[437] S. Dimopoulos, L. Susskind, Nucl. Phys. B155, 23, (1979); E. Eichten, K. Lane, Phys. Lett. B 90, 125 (1980).

[438] Some recent reviews are K. Lane, hep-ph/0202255 C. Hill and E. Simmons, Phys. Rep. 381, 235 (2003); R. S. Chivukula, M. Narain, J. Womersley, in Ref. [3].

[439] B. Holdom, Phys. Lett. B 150 (1985) 301; K Yamawaki, M. Bando, K. Matumoto, Phys. Rev. Lett. 56 (1986) 1335; T. Appelquist, D. Karabali, L.C.R. Wijewardhana, Phys. Rev. Lett. 57 (1986) 957; T. Appelquist and L.C.R. Wijewardhana, Phys. Rev. D 35 (1987) 774.

[440] T. Appelquist, J. Terning, Phys. Rev. D 50, 2116 (1994). 
[441] T. Appelquist and R. Shrock, in Neutrino Factories and Superbeams, NuFact03, A.I.P. Conf. Proc. 721 (A.I.P., New York, 2004), p. 261.

[442] T. Appelquist, M. Piai, and R. Shrock, Phys. Rev. D 69, 015002 (2004); T. Appelquist, N. Christensen, M. Piai, and R. Shrock, Phys. Rev. D 70, 093010 (2004).

[443] J. C. Pati and A. Salam, Phys. Rev. D 10, 275 (1974).

[444] E. Akhmedov, M. Lindner, E. Schnapka, J. Valle, Phys. Rev. D 53, 2752 (1996).

[445] S. P. Martin, Phys. Rev. D 44, 2892 (1991);

S. Antusch, J. Kersten, M. Lindner and M. Ratz, Nucl. Phys. B 658, 203 (2003) [arXiv:hep-ph/0211385].

[446] T. Appelquist, N. Christensen, M. Piai, and R. Shrock, Phys. Rev. D 70, 093010 (2004).

[447] T. Kaluza, Sitzungsber. d. Preuss. Akad. d. Wiss. Berlin, (1921) 966; O. Klein, Z. Phys. 37 (1926) 895.

[448] I. Antoniadis, Phys. Lett. B246 (1990) 377; J.D. Lykken, Phys. Rev. D54 (1996) 3693.

[449] E. Witten, Nucl. Phys. B471 (1996) 135; P. Hořava and E. Witten, Nucl. Phys. B460 (1996) 506; Nucl. Phys. B475 (1996) 94.

[450] N. Arkani-Hamed, S. Dimopoulos and G. Dvali, Phys. Lett. B429 (1998) 263; I. Antoniadis, N. Arkani-Hamed, S. Dimopoulos and G. Dvali, Phys. Lett. B436 (1998) 257.

[451] K.R. Dienes, E. Dudas and T. Gherghetta, Phys. Lett. B436 (1998) 55; Nucl. Phys. B537 (1999) 47.

[452] G.F. Giudice, R. Rattazzi and J.D. Wells, Nucl. Phys. B544 (1999) 3; T. Han, J.D. Jykken and R.-J. Zhang, Phys. Rev. D59 (1999) 105006; J.L. Hewett, hep-ph/9811356 E.A. Mirabelli, M. Perelstein and M.E. Peskin, Phys. Rev. Lett. 82 (1999) 2236; S. Nussinov and R. Shrock, Phys. Rev. D59 (1999) 105002; T.G. Rizzo, Phys. Rev. D59 (1999) 115010; P. Nath and M. Yamaguchi, Phys. Rev. D60 (1999) 116006; A. Mück, A. Pilaftsis and R. Rückl, Phys. Rev. D 65 (2002) 085037; hep-ph/0312186.

[453] K.R. Dienes, E. Dudas and T. Gherghetta, Nucl. Phys. B557 (1999) 25.

[454] N. Arkani-Hamed, S. Dimopoulos, G. Dvali and J. March-Russell, hep-ph/9811448.

[455] G. Dvali and A. Yu. Smirnov, Nucl. Phys. B563 (1999) 63.

[456] A. Pilaftsis, Phys. Rev. D60 (1999) 105023.

[457] J. Scherk and J.H. Schwarz, Phys. Lett. B82 (1979) 60; Nucl. Phys. B153 (1979) 61; P. Fayet, Phys. Lett. B159 (1985) 121; Nucl. Phys. B263 (1986) 649. 
[458] G. Bhattacharyya, H.-V. Klapdor-Kleingrothaus, H. Päs and A. Pilaftsis, Phys. Rev. D67 (2003) 113001.

[459] R.N. Mohapatra, S. Nandi and A. Perez-Lorenzana, Phys. Lett. B466 (1999) 115.

[460] R.N. Mohapatra and A. Perez-Lorenzana, Nucl. Phys. B576 (2000) 466.

[461] R. Barbieri, P. Creminelli and A. Strumia, Nucl. Phys. B585 (2000) 28; A. Ioannisian and J.W.F. Valle, Phys. Rev. D63 (2001) 073002; D.O. Caldwell, R.N. Mohapatra and S.J. Yellin, Phys. Rev. D64 (2001) 073001; K.R. Dienes and I. Sarcevic, Phys. Lett. B500 (2001) 133; A. de Gouvêa, G.F. Giudice, A. Strumia and K. Tobe, Nucl. Phys. B623 (2002) 395.

[462] A. Lukas, P. Ramond, A. Romanino and G.G. Ross, JHEP 0104 (2001) 010.

[463] H. Davoudiasl, P. Langacker and M. Perelstein, Phys. Rev. D65 (2002) 105015.

[464] H. S. Goh and R. N. Mohapatra, Phys. Rev. D 65, 085018 (2002) hep-ph/0110161.

[465] G. Mclaughlin and J. N. Ng, Phys. Rev. D 63, 053002 (2001).

[466] H. Yu, S.-P. Ng and R. N. Mohapatra, Phys.Rev.D70 057301 (2004).

[467] D.O. Caldwell, R.N. Mohapatra and S.J. Yellin, Phys. Rev. D64 (2001) 073001.

[468] A. Ioannisian and A. Pilaftsis, Phys. Rev. D62 (2000) 066001.

[469] A.E. Faraggi and M. Pospelov, Phys. Lett. B458 (1999) 237; K. Agashe and G. H. Wu, Phys. Lett. B498 (2001) 230; B. He, T.P. Cheng and L.F. Li, Phys. Lett. B553 (2003) 277.

[470] Q.-H. Cao, S. Gopalakrishna and C.P. Yuan, hep-ph/0312339. G. Moreau, hep-ph/0407177; J. L. Hewett, P. Roy and S. Roy, Phys. Rev. D 70, 051903 (2004).

[471] H.V. Klapdor-Kleingrothaus and U. Sarkar, Mod. Phys. Lett. A16 (2001) 2469.

[472] R.N. Mohapatra, A. Perez-Lorenzana and C.A. de S. Pires, Phys. Lett. B491 (2000) 143.

[473] S.J. Huber and Q. Shafi, Phys. Lett. B544 (2002) 295.

[474] T.D.Lee and C.N.Yang, Phys rev 98 , 1501 (1955).

[475] L.B.Okun, Sov J Nucl Phys , 10206 (1969); For a review see A.D.Dolgov , Phys Rept 320, 1 (1999).

[476] E.G.Adelberger, B.R.Heckel and A.E.Nelson, hep-ph/0307284.

[477] A.S.Joshipura and S. Mohanty , Phys. Lett. B584 103 (2004)(hep-ph/0310210).

[478] J.A.Grifols and E. Masso, Phys Lett B 579, 123(2004). 
[479] R.Foot, Mod Phys Lett A 6, 527 (1991); X.-G.He, G.C.Joshi , H.Lew and R.R.Volkas, Phys Rev D44, 2118 (1991); R.Foot et al, Phys Rev D 50, 4571(1994).

[480] H. W. Zaglaur and K. H. Schwarzer, Z. Phys. C40 273(1998); A. Bueno, M. Campanelli and A. Rubbia, Nucl. Phys. 589577 (2000).

[481] K. Hagiwara et al. [Particle Data Group Collaboration], Phys. Rev. D 66, 010001 (2002).

[482] H. Murayama, hep-ph/0307127. For more recent (and more stringent bounds) see A. de Gouvêa and C. Peña-Garay, hep-ph/0406301. Equivalent bounds in the atmospheric sector can be found in A. de Gouvêa, Nucl. Phys. Proc. Suppl. 143, 167 (2005) [arXiv hep-ph/0408246; H. Minakata, H. Nunokawa, W.J.C. Teves and R. Zukanovich Funchal, Phys. Rev. D 71, 013005 (2005) [arXiv hep-ph/0407326].

[483] H. Murayama and T. Yanagida, Phys. Lett. B 520, 263 (2001) hep-ph/0010178.

[484] G. Barenboim, L. Borissov, J. Lykken and A. Y. Smirnov, JHEP 0210, 001 (2002) hep-ph/0108199.

[485] A. Strumia, Phys. Lett. B 539, 91 (2002) hep-ph/0201134.

[486] G. Barenboim, L. Borissov and J. Lykken, Phys. Lett. B 534, 106 (2002) hep-ph/0201080.

[487] G. Barenboim, L. Borissov and J. Lykken, hep-ph/0212116.

[488] M. C. Gonzalez-Garcia, M. Maltoni and T. Schwetz, Phys. Rev. D 68, 053007 (2003) hep-ph/0306226.

[489] V. Barger, D. Marfatia and K. Whisnant, Phys. Lett. B 576, 303 (2003) hep-ph/0308299.

[490] O. W. Greenberg, Phys. Rev. Lett. 89, 231602 (2002) hep-ph/0201258.

[491] A. de Gouvêa, Phys. Rev. D 66, 076005 (2002) hep-ph/0204077.

[492] A. V. Kostelecky and M. Mewes, hep-ph/0308300; S. Choubey and S. F. King, Phys. Lett. B 586, 353 (2004) hep-ph/0311326|.

[493] G. Barenboim and N. E. Mavromatos, hep-ph/0404014.

[494] S. Choubey and S. F. King, Phys. Lett. B 586 (2004) 353 [arXiv:hep-ph/0311326].

[495] J. Ellis, J. Hagelin, D.V. Nanopoulos and M. Srednicki, Nucl. Phys. B241, 381 (1984).

[496] F. Benatti and R. Floreanini, Phys. Lett. B 468 (1999) 287 hep-ph/9910508.

[497] E. Lisi, A. Marrone and D. Montanino, Phys. Rev. Lett. 85, 1166 (2000) hep-ph/0002053. 
[498] [NuTeV Collaboration] G. P. Zeller et al., Phys. Rev. Lett. 88, 091802 (2002) hep-ex/0110059; Phys. Rev. D 65, 111103 (2002) hep-ex/0203004; K. S. McFarland et al., hep-ex/0205080; G. P. Zeller et al., hep-ex/0207052.

[499] C. H. Llewellyn Smith, Nucl. Phys. B 228, 205 (1983).

[500] M. S. Chanowitz, Phys. Rev. D 66, 073002 (2002) hep-ph/0207123.

[501] The LEP Collaborations, the LEP Electroweak Working Group, and the SLD Heavy Flavor and Electroweak Groups, CERN-EP/2003-091, hep-ex/0312023.

[502] S. Davidson, S. Forte, P. Gambino, N. Rius and A. Strumia, JHEP 0202, 037 (2002) hep-ph/0112302; S. Davidson, hep-ph/0209316; P. Gambino, hep-ph/0211009.

[503] B. A. Dobrescu and R. K. Ellis, hep-ph/0310154.

[504] K. P. O. Diener, S. Dittmaier and W. Hollik, hep-ph/0310364.

[505] P. Gambino, hep-ph/0311257.

[506] W. Loinaz and T. Takeuchi, Phys. Rev. D 60, 115008 (1999) hep-ph/9903362.

[507] E. Ma, D. P. Roy and S. Roy, Phys. Lett. B 525, 101 (2002) hep-ph/0110146|; E. Ma and D. P. Roy, Phys. Rev. D 65, 075021 (2002) hep-ph/0111385|; Nucl. Phys. B 644, 290 (2002) hep-ph/0206150.

[508] M. Gronau, C. N. Leung and J. L. Rosner, Phys. Rev. D 29, 2539 (1984); J. Bernabeu, A. Santamaria, J. Vidal, A. Mendez and J. W. Valle, Phys. Lett. B 187, 303 (1987); K. S. Babu, J. C. Pati and X. Zhang, Phys. Rev. D 46, 2190 (1992); W. J. Marciano, Phys. Rev. D 60, 093006 (1999) hep-ph/9903451; A. de Gouvêa, G. F. Giudice, A. Strumia and K. Tobe, Nucl. Phys. B 623, 395 (2002) hep-ph/0107156; K. S. Babu and J. C. Pati, hep-ph/0203029.

[509] L. N. Chang, D. Ng and J. N. Ng, Phys. Rev. D 50, 4589 (1994) hep-ph/9402259;;

[510] W. Loinaz, N. Okamura, T. Takeuchi and L. C. R. Wijewardhana, Phys. Rev. D 67, 073012 (2003) hep-ph/0210193; T. Takeuchi, hep-ph/0209109; T. Takeuchi, W. Loinaz, N. Okamura and L. C. R. Wijewardhana, hep-ph/0304203.

[511] W. Loinaz, N. Okamura, S. Rayyan, T. Takeuchi and L. C. R. Wijewardhana, Phys. Rev. D 68, 073001 (2003) hep-ph/0304004.

[512] M. E. Peskin and T. Takeuchi, Phys. Rev. Lett. 65, 964 (1990); Phys. Rev. D 46, 381 (1992), J. L. Hewett, T. Takeuchi and S. Thomas, hep-ph/9603391.

[513] S. L. Glashow, hep-ph/0301250.

[514] B. W. Lee, S. Pakvasa, R. E. Shrock and H. Sugawara, Phys. Rev. Lett. 38, 937 (1977).

[515] S. Ahmad et al., Phys. Rev. D 38 (1988) 2102; 
[516] F. Simkovic, V. E. Lyubovitskij, T. Gutsche, A. Faessler and S. Kovalenko, Phys. Lett. B 544, 121 (2002) hep-ph/0112277|; R. Kitano, M. Koike and Y. Okada, Phys. Rev. D 66, 096002 (2002) hep-ph/0203110|; R. Kitano, M. Koike, S. Komine and Y. Okada, Phys. Lett. B 575, 300 (2003) hep-ph/0308021.

[517] L. Willmann et al., Phys. Rev. Lett. 82, 49 (1999) hep-ex/9807011.

[518] A. Halprin, Phys. Rev. Lett. 48 (1982) 1313; T. E. Clark and S. T. Love, Mod. Phys. Lett. A 19, 297 (2004) hep-ph/0307264.

[519] W. Loinaz, N. Okamura, S. Rayyan, T. Takeuchi and L. C. R. Wijewardhana, hep-ph/0403306.

[520] S. Ritt [MUEGAMMA Collaboration], Nucl. Instrum. Meth. A 494 (2002) 520. See also the MEG Collaboration website at http://meg.web.psi.ch/.

[521] J. L. Popp [MECO Collaboration], Nucl. Instrum. Meth. A 472, 354 (2000) hep-ex/0101017; M. Hebert [MECO Collaboration], Nucl. Phys. A 721, 461 (2003).

[522] E. Sichtermann [g-2 Collaboration], eConf C030626, SABT03 (2003) hep-ex/0309008.

[523] E. Ma and D. P. Roy, Phys. Rev. D 65, 075021 (2002) hep-ph/0111385; K. S. Babu and J. C. Pati, Phys. Rev. D 68, 035004 (2003) hep-ph/0207289|; T. Fukuyama, T. Kikuchi and N. Okada, Phys. Rev. D 68, 033012 (2003) hep-ph/0304190|.

[524] P. Langacker, AIP Conf. Proc. 698, 1 (2004) hep-ph/0308145.

[525] H. Abele et al., Phys. Rev. Lett. 88, 211801 (2002) hep-ex/0206058; Eur. Phys. J. C 33, 1 (2004) hep-ph/0312150.

[526] B. Tipton et al., AIP Conf. Proc. 539, 286 (2000). 